\title{
Measurement of the Top Quark Mass in the Di-lepton Channel using the Dalitz-Goldstein Method
}

\author{
A dissertation \\ submitted by \\ Matthew Frederick Hare \\ in partial fulfillment of the requirements \\ for the degree of \\ Doctor of Philosophy \\ in \\ Physics

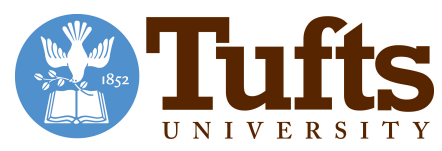

October 2010

Advisor: Professor Krzysztof Sliwa 

This dissertation is dedicated to the memory of my father

\title{
David James Hare
}

October 15, 1940 - November 22, 2009

\author{
and my brother
}

\section{Timothy David Hare}

March 20, 1971 - October 24, 2009 


\title{
Measurement of the Top Quark Mass in the Di-lepton Channel using the Dalitz-Goldstein Method
}

\author{
by Matthew Frederick Hare \\ Advisor: Professor Krzysztof Sliwa
}

\begin{abstract}
This dissertation describes a measurement of the mass of the top quark using a method developed by G. Goldstein and R.H. Dalitz. It is based on $2.0 \mathrm{fb}^{-1}$ of data collected by the Collider Detector Facility at Fermi National Accelerator Laboratories. Di-lepton events were observed from colliding protons with anti-protons with $\sqrt{s}=1.96 \mathrm{TeV}$ in the Tevatron Collider. A total of 145 candidate events were observed with 49 expected to be from background. These events include two neutrinos which elude detection. The method begins by assuming an initial top quark mass and solves for the neutrino momenta using a geometrical construction. The method samples over a range of likely top quark masses choosing the most consistent mass via a likelihood function. An important distinguishing feature of this method from others is its lack of dependence on the missing transverse energy, a quantity that is poorly measured by the experiment. This analysis determines the top quark mass to be $M_{\text {top }}=172.3 \pm 3.4$ (stat.) \pm 2.0 (syst.) $\mathrm{GeV} / c^{2}\left(M_{\text {top }}=170.5 \pm\right.$ 3.7 (stat.) \pm 1.8 (syst.) $\mathrm{GeV} / c^{2}$ with $b$-tagging) .
\end{abstract}




\section{Acknowledgments}

I would like to thank my advisor, Professor Krzysztof Sliwa, whose guidance and support over the years have helped to make this dissertation a reality.

I would also like to thank the Tufts Physics Department, especially the professors and staff in the High Energy Department: Gary Golstein, thank you for you work in developing this method, Tony Mann, for an enjoyable E\&M course as well as continued motivation; Bill Oliver, for his ongoing support and sympathetic ear to grad student life woes; Roger Tobin, for a great QM course. I would also like to thank Austin Napier for help and support in keeping our computers running. Thank you Simona Rolli for your advice and help at FNAL. And huge thank you for Jackie DiMichele and Jean Intoppa for all your help in arranging travel to FNAL and ordering so many supplies over the years.

To Benjamin Whitehouse, Hao Sun, and Dan Ryan, thank you for all the advice and mutual support. Especially to Ben whose expertise in $\mathrm{C}++$ helped me wade through the miles of CDF code and correct my own bugs.

From CDF I would like to thank Monica Tecchio, George Valev, and Erik Brubaker. Your advice helped me immensely.

I would like to give a special thanks to Jennifer Bell for helping me proofread this document. Thank you for your patience and friendship.

Thank you to Robert Carey and family. Your friendship, help in tackling some of the problems in this work, the free laundry, the meals, and always entertaining company for as long as I have known you have helped to keep me on track in both my grad life and my everyday life. I am happy to call you my friends and look forward to the years to come. You are like a second family to me.

To the Tufts Ballroom Team I would like to express how much fun I have had with you in my last two years at Tufts. Especially to Vicki, Zach, Elena, Ben, Montana, Tracy, Emily, Alex, Dana, Robyn, Scott, Jim, James, Hyejo, David, Jonathan, Lumay, and all of my newbies (bronze now!). I consider you all my friends and hope to remain so forever. I have enjoyed learning from all of you and teaching all of you. Keep Dancing!

To my Mother and Father, thank you for all the love and support. You have always been there for me and believed in me. I could not have asked for better parents. For my brothers, 
Timothy and Daniel, and my sister, Bonnie, thank you for all that you have taught me. It makes me happy to know how strong of a family I have. I am grateful to be part of it. I love all of you!

And to all my friends, thank you for being part of my life. Without my friends, I could not survive in this world. 


\section{Contents}

Abstract $\quad$ ii

Acknowledgments

List of Tables $\quad$ xiii

List of Figures $\quad$ Xvii

Chapter 1 - Top Quark Physics 2

1.1 Introduction . . . . . . . . . . . . . . . . . . . . . . 2

1.2 The Fundamental Building Blocks . . . . . . . . . . . . . . . . . . . 2

1.3 Leptons . . . . . . . . . . . . . . . . . . . . . . . . 4

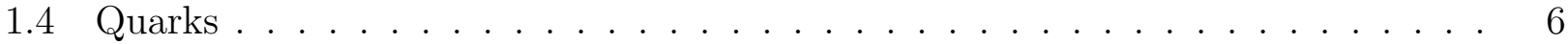

1.5 Gauge Bosons . . . . . . . . . . . . . . . . . . . . . . . . . . 7

1.6 The Standard Model . . . . . . . . . . . . . . . . . . . . . . . . . . . . 9

1.7 The Top Quark . . . . . . . . . . . . . . . . . . . . . 14

Chapter 2 - The Experimental Apparatus $\quad 19$

2.1 FNAL and CDF . . . . . . . . . . . . . . . . . . 19

2.2 The Tevatron . . . . . . . . . . . . . . . . . . . . . . 19

2.3 The Collider Detector at Fermilab . . . . . . . . . . . . . . . . . . . . . . 21

2.4 Event Triggering . . . . . . . . . . . . . . . . . . . . . 23

Chapter 3 - The Jets $\quad 26$

3.1 Introduction . . . . . . . . . . . . . . . . . . 26

3.2 Jet Reconstruction . . . . . . . . . . . . . . . . . . . . . . . . . 26

3.3 Standard Jet Energy Corrections . . . . . . . . . . . . . . . . . . . . 27

3.3.1 Level 1 Relative Energy Correction . . . . . . . . . . . . . . . . . 27

3.3.2 Level 2 Central Calorimeter Stability . . . . . . . . . . . . . . 27

3.3.3 Level 3 Scale Correction . . . . . . . . . . . . . . . . . 27

3.3.4 Level 4 Multiple Interactions Correction . . . . . . . . . . . . . . . . 28 
3.3.5 Level 5 Absolute Energy Correction . . . . . . . . . . . . . . . . . . . 28

3.3.6 Level 6 Underlying Event Subtraction . . . . . . . . . . . . . . . . . . . . 29

3.3.7 Level 7 Out-of-Cone Correction . . . . . . . . . . . . . . . . . . . 30

3.3.8 Level 8 Splash Out Correction . . . . . . . . . . . . . . . . . . . . . 30

$\begin{array}{ll}\text { Chapter } 4 \text { - The Di-lepton Channel } & 31\end{array}$

4.1 Introduction . . . . . . . . . . . . . . . . . . . . 31

4.2 Top Mass Measurements in the Di-lepton Channel . . . . . . . . . . . . . . . . . 31

4.2 .1 The Template Method . . . . . . . . . . . . . . . . . . . . . . . 31

4.2 .2 The Matrix Element Method . . . . . . . . . . . . . . . . . . . . . . 32

4.3 Event Selection in the Di-lepton Channel . . . . . . . . . . . . . . . . . . 33

4.3 .1 Electron Selection Cuts . . . . . . . . . . . . . . . . . . . 33

4.3.2 Muon Selection Cuts . . . . . . . . . . . . . . . . . . . . 35

4.3 .3 Jet Selection Cuts . . . . . . . . . . . . . . . . . . . . . . . . 38

4.3.4 Event Selection Cuts . . . . . . . . . . . . . . . . . . . . . 38

4.3 .5 Trigger Requirements . . . . . . . . . . . . . . . . . . . . . . . . . 39

4.4 Backgrounds for the Di-lepton Channel . . . . . . . . . . . . . . . . . . . . . . 39

4.4 .1 Dibosons . . . . . . . . . . . . . . . . . . . . . . . . . . . . . . . 39

4.4 .2 Drell-Yan . . . . . . . . . . . . . . . . . . . . . . . . . . 41

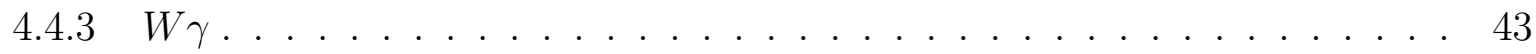

4.4 .4 Fakes . . . . . . . . . . . . . . . . . . . . . 43

4.4 .5 Background Summary . . . . . . . . . . . . . . . . . 44

Chapter 5 - The Dalitz and Goldstein Method 46

5.1 Introduction . . . . . . . . . . . . . . . . . . . 46

5.2 Geometrical Construction $\ldots \ldots \ldots 4 \ldots$

5.3 The Likelihood . . . . . . . . . . . . . . . . . . . . . . . . . 50

5.4 MET Probability . . . . . . . . . . . . . . . . . . . . 55

5.5 The Joint Likelihood . . . . . . . . . . . . . . . . . . . . 55

5.6 The Construction of the Pseudo-Experiments . . . . . . . . . . . 56

5.6 .1 The RMS Correction . . . . . . . . . . . . . . . . . 56 
5.6 .2 The Mapping Function . . . . . . . . . . . . . . . . . . . . . 58

5.6.3 Additional Types of Combination Selection . . . . . . . . . . . . . 68

5.6 .4 Contributions from Backgrounds . . . . . . . . . . . . . . 70

5.7 Statistical Error . . . . . . . . . . . . . . . . . . . . . . . 73

5.8 Sanity Checks . . . . . . . . . . . . . . . . . . . . . . . . . . . . . 77

5.9 Blind Samples . . . . . . . . . . . . . . . . . . . . . . . . . . . . . 80

$\begin{array}{lr}\text { Chapter } 6 \text { - Systematic Errors } & 81\end{array}$

6.1 Introduction . . . . . . . . . . . . . . . . . . . . 81

6.2 Jet Energy Scale . . . . . . . . . . . . . . . . . . . . . . . . . . . . 81

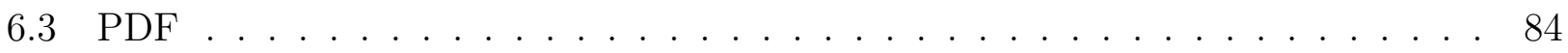

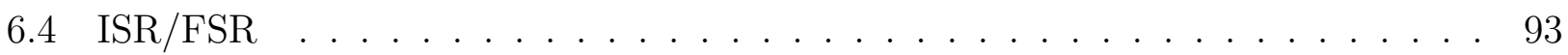

6.5 MC Generator . . . . . . . . . . . . . . . . . . . . . . . . . . . 94

6.6 Background Fraction . . . . . . . . . . . . . . . . . . . . . . . 94

$6.7 \quad b$-Jet Energy Scale . . . . . . . . . . . . . . . . . . . . . . . . . . . . 98

$6.8 \quad$ Lepton $P_{T} \ldots \ldots \ldots \ldots \ldots \ldots$

6.9 Pile-Up . . . . . . . . . . . . . . . . . . . . . . . . . . . . . . . 100

6.10 Color Reconnection . . . . . . . . . . . . . . . . . . . . . . . . . 102

6.11 Systematic Summary . . . . . . . . . . . . . . . . . . . . . 103

$\begin{array}{lr}\text { Chapter } 7 \text { - } b \text {-Tagging } & 105\end{array}$

7.1 Introduction . . . . . . . . . . . . . . . . . . . . . . . . . . 105

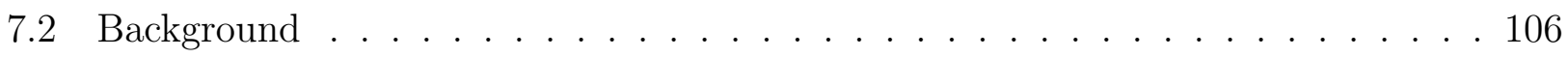

7.3 Mapping Functions . . . . . . . . . . . . . . . . . . . . . 106

7.4 Statistical Errors . . . . . . . . . . . . . . . . . . . . . . . 109

7.5 Systematic Errors . . . . . . . . . . . . . . . . . . . . . . . . . 109

7.5.1 Jet Energy Scale . . . . . . . . . . . . . . . . . . . . . . . 110

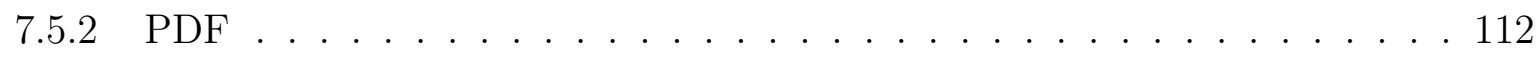

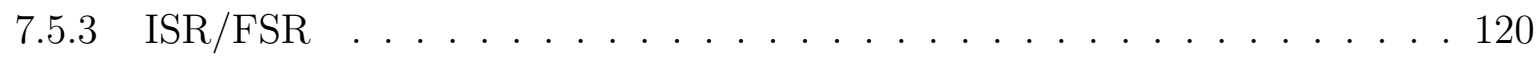

7.5 .4 MC Generator . . . . . . . . . . . . . . . . . . . . . . . 121

7.5.5 Background Fraction . . . . . . . . . . . . . . . . 121 
7.5.6 b-Jet Energy Scale . . . . . . . . . . . . . . . . . . 123

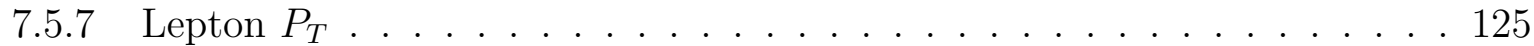

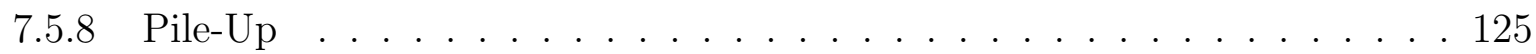

7.5.9 Color Reconnection . . . . . . . . . . . . . . . . 126

7.5.10 Systematic Summary . . . . . . . . . . . . . . . . 126

$\begin{array}{lr}\text { Chapter } 8 \text { - Results } & 128\end{array}$

8.1 Introduction . . . . . . . . . . . . . . . . . . . . . . . 128

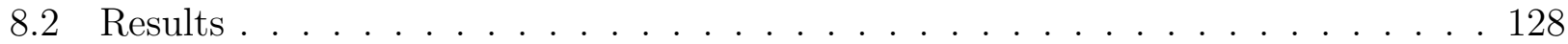

8.3 Comparison of Results . . . . . . . . . . . . . . . . . . . . . 129

$\begin{array}{ll}\text { A The Bootstrap Technique } & 132\end{array}$

B Methods for Calculating Systematic Uncertainties 133 


\section{List of Tables}

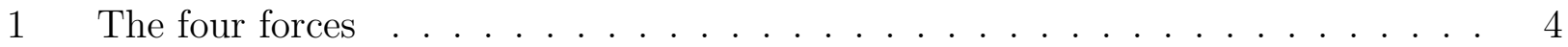

2 The three generations of the leptons and quarks . . . . . . . . . . . 4

3 Properties of the leptons and neutrinos. Be careful not to confuse the symbols $e^{-}$and $e^{+}$, which stand for the electron and positron, with the charges $-e$ and $+e$ which are $\pm 1.602 \times 10^{-19}$ C. $[1] \ldots \ldots \ldots \ldots \ldots$

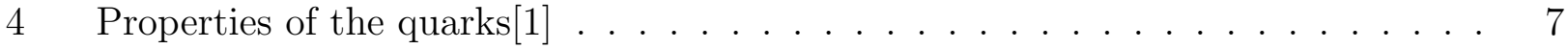

5 Properties of the three gauge bosons and the graviton[1] . . . . . . . . 8

6 Various decay modes of $t \bar{t}$ events . . . . . . . . . . . . . . . . . 15

7 Additional energy per vertex by cone size. $[2] \ldots \ldots \ldots 28$

8 Parameterization of the Absolute Energy Correction[2] . . . . . . . . . . . . . 29

9 Underlying Event Subtraction energy by cone size.[2] . . . . . . . . . . . . 30

10 Out-of-Cone Correction parameterization $[2] \ldots \ldots$. . . . . . . . . . 30

11 Electron selection cuts for CDF di-lepton events . . . . . . . . . . . . . . . 36

12 Muon selection cuts for CDF di-lepton events . . . . . . . . . . . . . . 38

13 Signal and background event expectations predicted by the Standard Model.[3] . 45

14 Signal and background event expectations predicted by the Standard Model by

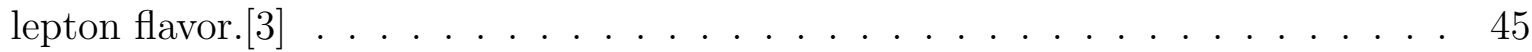

15 Correction factors to the width of the top mass joint distribution. . . . . . . . 73

16 Likelihood only mass distribution means, uncertainty on the means, and systematic uncertainties from each of the jet energy correction levels. Additionally, the sum in quadrature for all the individual levels. . . . . . . . . . . . . . . . 82

17 Likelihood added mass distribution means, uncertainty on the means, and systematic uncertainties from each of the jet energy correction levels. Additionally, the sum in quadrature for all the individual levels. . . . . . . . . . . . . . .

182 leading jets mass distribution means, uncertainty on the means, and systematic uncertainties from each of the jet energy correction levels. Additionally, the sum in quadrature for all the individual levels. . . . . . . . . . . . . . . . . . . 83 
192 leading jets added mass distribution means, uncertainty on the means, and systematic uncertainties from each of the jet energy correction levels. Additionally, the sum in quadrature for all the individual levels. . . . . . . . . . . . . . 83

20 Likelihood only mass distribution means and uncertainty on the means from each of the $46 \mathrm{PDFs} \ldots \ldots \ldots$. . . . . . . . . . . . . . . . . . . 85

21 Likelihood added mass distribution means and uncertainty on the means from each of the 46 PDFs . . . . . . . . . . . . . . . . . . . . . . 86

222 leading jets mass distribution means and uncertainty on the means from each of the $46 \mathrm{PDFs} \ldots \ldots \ldots \ldots$. . . . . . . . . . . . . . . . . . . . . . . . .

232 leading jets mass distribution means and uncertainty on the means from each of the $46 \mathrm{PDFs} \ldots \ldots \ldots \ldots \ldots$

24 Likelihood only uncertainties for the different PDFs . . . . . . . . . . . . . . . 89

25 Likelihood added uncertainties for the different PDFs . . . . . . . . . . . . . . . 90

262 leading jets uncertainties for the different PDFs . . . . . . . . . . . . . 91

272 leading jets added uncertainties for the different PDFs . . . . . . . . . . . 92

28 Mass distribution means and the uncertainty on the means for the IFS/FSR systematic samples. . . . . . . . . . . . . . . . . . . . . 93

29 Difference of the ISR/FSR systematic samples from the nominal sample. . . . . 93

30 Mass distribution means and the uncertainty on the means for the PYTHIA and HERWIG samples as well as the difference of the HERWIG samples from the nominal (PYTHIA) sample. . . . . . . . . . . . . . . . . . . 94

31 Likelihood only mass distribution means and the systematic uncertainties for each of the shifted backgrounds and the sum in quadrature of the systematic

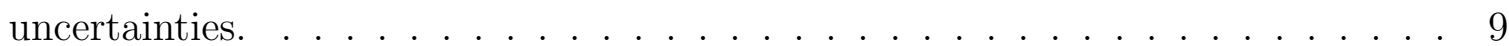

32 Likelihood added mass distribution means and the systematic uncertainties for each of the shifted backgrounds and the sum in quadrature of the systematic uncertainties. . . . . . . . . . . . . . . . . . 96

332 leading jets mass distribution means and the systematic uncertainties for each of the shifted backgrounds and the sum in quadrature of the systematic uncertainties. . . . . . . . . . . . . . . . . . . . 96 
342 leading jets mass distribution means and the systematic uncertainties for each of the shifted backgrounds and the sum in quadrature of the systematic uncertainties. . . . . . . . . . . . . . . . . . . . 97

35 Likelihood only mass distribution means and the uncertainty on the means for the $b$-Jet systematic studies. . . . . . . . . . . . . . . . . . . . . . . . . . . 98

36 Likelihood added mass distribution means and the uncertainty on the means for the $b$-Jet systematic studies. . . . . . . . . . . . . . . . . . . . . . . . . . . . 99

372 leading jets mass distribution means and the uncertainty on the means for the b-Jet systematic studies. . . . . . . . . . . . . . . . . . . . . . 99

382 leading jets added mass distribution means and the uncertainty on the means for the b-Jet systematic studies. . . . . . . . . . . . . . . . . . . . . . 99

39 Mass distribution means and the uncertainty on the means for the shifted lepton energy systematic study. . . . . . . . . . . . . . . . . . . . 100

40 The values of the various variables that go into calculating the systematic uncertainty due to pile-up. . . . . . . . . . . . . . . . . . . . . 102

41 Mass distribution means and the uncertainty on the means for the two color reconnection samples as well as the difference. . . . . . . . . . . . . . . 103

42 Systematic errors from various sources . . . . . . . . . . . . . . . . . . . . . . 104

43 Signal and background event expectations predicted by the Standard Model with b-tagging. . . . . . . . . . . . . . . . . . 106

44 Correction factors to the width of the top mass joint distribution with $b$-tagging. 109

45 Likelihood only mass distribution means, uncertainty on the means, and systematic uncertainties from each of the jet energy correction levels. Additionally, the sum in quadrature for all the individual levels. . . . . . . . . . . . . . . . . . . . 110

46 Likelihood added mass distribution means, uncertainty on the means, and systematic uncertainties from each of the jet energy correction levels. Additionally, the sum in quadrature for all the individual levels. . . . . . . . . . . . . . . . . 110

472 leading jets mass distribution means, uncertainty on the means, and systematic uncertainties from each of the jet energy correction levels. Additionally, the sum in quadrature for all the individual levels. . . . . . . . . . . . . . . . . . . . 111 
482 leading jets added mass distribution means, uncertainty on the means, and systematic uncertainties from each of the jet energy correction levels. Additionally, the sum in quadrature for all the individual levels. . . . . . . . . . . . . . . . . . 111

49 Likelihood only mass distribution means and uncertainty on the means from each of the $46 \mathrm{PDFs} \ldots \ldots \ldots \ldots$. . . . . . . . . . . . . . . . . . 112

50 Likelihood added mass distribution means and uncertainty on the means from

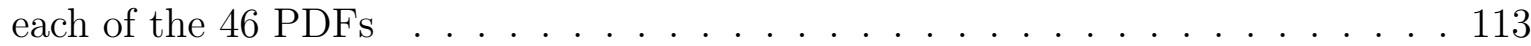

512 leading jets mass distribution means and uncertainty on the means from each of the $46 \mathrm{PDFs} \ldots \ldots \ldots \ldots$

522 leading jets mass distribution means and uncertainty on the means from each of the $46 \mathrm{PDFs} \ldots \ldots \ldots \ldots \ldots \ldots$

53 Likelihood only uncertainties for the different PDFs . . . . . . . . . . . . 116

54 Likelihood added uncertainties for the different PDFs . . . . . . . . . . . . . . 117

552 leading jets uncertainties for the different PDFs . . . . . . . . . . . . . 118

562 leading jets added uncertainties for the different PDFs . . . . . . . . . . . 119

57 Mass distribution means and the uncertainty on the means for the IFS/FSR systematic samples. . . . . . . . . . . . . . . . . . . 120

58 Difference of the ISR/FSR systematic samples from the nominal sample. . . . . 120

59 Mass distribution means and the uncertainty on the means for the PYTHIA and HERWIG samples as well as the difference of the HERWIG samples from the nominal (PYTHIA) sample. . . . . . . . . . . . . . . . . . . 121

60 Likelihood only mass distribution means and the systematic uncertainties for each of the shifted backgrounds and the sum in quadrature of the systematic uncertainties. . . . . . . . . . . . . . . . . . . . . 121

61 Likelihood added mass distribution means and the systematic uncertainties for each of the shifted backgrounds and the sum in quadrature of the systematic uncertainties. . . . . . . . . . . . . . . . . . 122

622 leading jets mass distribution means and the systematic uncertainties for each of the shifted backgrounds and the sum in quadrature of the systematic uncertainties. . . . . . . . . . . . . . . . . . . . 122 
632 leading jets mass distribution means and the systematic uncertainties for each of the shifted backgrounds and the sum in quadrature of the systematic uncer-

64 Likelihood only mass distribution means and the uncertainty on the means for the $b$-Jet systematic studies. . . . . . . . . . . . . . . . . . . . . . . 123

65 Likelihood added mass distribution means and the uncertainty on the means for the $b$-Jet systematic studies. . . . . . . . . . . . . . . . . . . . . . . . . . . 124

662 leading jets mass distribution means and the uncertainty on the means for the b-Jet systematic studies. . . . . . . . . . . . . . . . . . . . . . . . . . 124

672 leading jets added mass distribution means and the uncertainty on the means for the $b$-Jet systematic studies. . . . . . . . . . . . . . . . . . . . . . . 124

68 Mass distribution means and the uncertainty on the means for the shifted lepton energy systematic study. . . . . . . . . . . . . . . . . . . . 125

69 The values of the various variables that go into calculating the systematic uncertainty due to pile-up. . . . . . . . . . . . . . . . . 126

70 Mass distribution means and the uncertainty on the means for the two color reconnection samples as well as the difference. . . . . . . . . . . . . . 126

71 Systematic errors from various sources . . . . . . . . . . . . . . . 127

72 Results without any corrections. (Raw) . . . . . . . . . . . . . 128

73 Final results with corrections. . . . . . . . . . . . . . . . . . 129 


\section{List of Figures}

1 Feynman diagram illustrating $t \bar{t}$ production via $q \bar{q}$ annihilation in the di-lepton

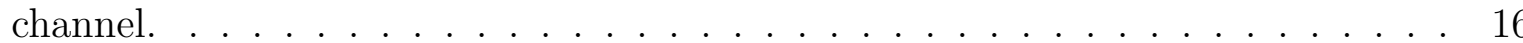

2 Feynman diagram illustrating $t \bar{t}$ production via gluon fusion in the di-lepton channel. . . . . . . . . . . . . . . . . . . . . 16

3 The green band in this plot shows the allowed range of masses for the higgs boson given a range of top and $\mathrm{W}$ boson masses. The blue oval shows the allowed region given measurements of the top and W masses from LEP2 and the Tevatron. As more precise measurements of the $\mathrm{W}$ and top masses are made, the overlap between the green band and the blue oval may change and possibly disappear all together.[4] . . . . . . . . . . . . . . . . . . . 1

4 Similar to the previous plot, this shows the regions where the current measured values of the top and $\mathrm{W}$ masses agree with modern theories that allow for the existence of the higgs boson. It is clear that a light top mass favors the MSSM

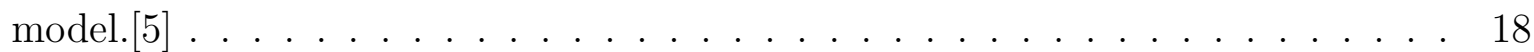

5 Graphical representation of proton and anti-proton beams at Fermilab $[6]$. . . . 20

6 One barrel of the SVXII[6] . . . . . . . . . . . . . . . . . . . . . . 21

7 Cross-section of the collider detector at Fermilab $[6]$. . . . . . . . . . . . . . . . 22

8 Block diagram of the CDF Run II trigger system $[6] \ldots \ldots$. . . . . . . . . . . 25

9 The two spheres of radii $R_{W}$ and $R_{\nu}$, whose centers are separated by $\vec{P}_{t}$, but do not intersect. . . . . . . . . . . . . . . . . . . 48

10 The two spheres of radii $R_{W}$ and $R_{\nu}$, which intersect at a point. $\mathrm{O}$ is the point where $E_{t}-E_{0}=0$, the minimum energy allowed for the top quark as determined by the kinematics of the event. . . . . . . . . . . . . . . . . . . . . . 49

11 The two spheres of radii $R_{W}$ and $R_{\nu}$, showing a circle of intersection. . . . . . 50

12 The two spheres of radii $R_{W}$ and $R_{\nu}$ showing a circle of intersection with constant energy $E_{t} \ldots \ldots \ldots \ldots \ldots \ldots \ldots$ 
13 The two spheres of radii $R_{W}$ and $R_{\nu}$ showing a circle of intersection with constant energy $E_{t}$. Also seen here is a second circle of constant $E_{t}$ resulting from the intersection of two other spheres (not drawn). Both circles begin to form the paraboloid surface. . . . . . . . . . . . . . . . . . 52

14 The two spheres of radii $R_{W}$ and $R_{\nu}$ showing a circle of intersection with constant energy $E_{t}$. Assuming a constant $M_{t}$ confines $P_{t}$ to a conic section of the paraboloid which is an ellipse. . . . . . . . . . . . . . . . 53

15 RMS correction factor as a function of top mass for signal-only events. . . . . . 59

16 RMS correction factor as a function of top mass for signal and background events. 60

17 Mapping function from signal-only MC truth tables with only the correct com-

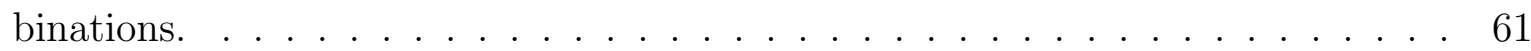

18 Mapping function from signal-only MC truth tables with the flipped combinations only. . . . . . . . . . . . . . . . . . . . . 61

19 Mapping function from signal-only MC truth tables (Left). Delta, Delta Width, Pull, and Pull Widths for the signal MC truth table data samples after corrections have been applied from the mapping function (Right) . . . . . . . . . . . . . 62

20 Mapping function from signal-only MC matchable events using only the correct combination. . . . . . . . . . . . . . . . . . . 63

21 Mapping function from signal-only MC matchable events using only the flipped combination. . . . . . . . . . . . . . . . . . . 64

22 Mapping function from signal-only MC matchable events using only a wrong

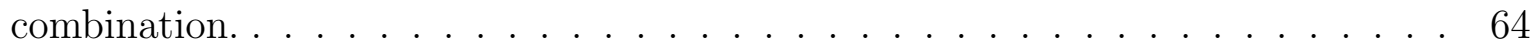

23 Mapping function from signal-only MC matchable events using a combination chosen by its likelihood (Left). Delta, Delta Width, Pull, and Pull Widths (Right). 65

24 Mapping function from signal-only MC events using only the correct combination. 66

25 Mapping function from signal-only MC events using only the flipped combination. 66

26 Mapping function from signal-only MC events using only a wrong combination. . 67

27 Mapping function from signal-only MC events using a combination chosen by its likelihood (Left). Delta, Delta Width, Pull, and Pull Widths (Right). . . . . . 67

28 Mapping functions, Deltas, Delta Widths, Pulls, and Pull Widths for the signal MC data samples where the most probable combination is added to its "twin". 
29 Mapping functions, Deltas, Delta Widths, Pulls, and Pull Widths for the signal MC data samples where the most probable combination is selected out of the combinations which contain both of the leading jets.

30 Mapping functions, Deltas, Delta Widths, Pulls, and Pull Widths for the signal MC data samples where the most probable combination is selected out of the combinations which contain both of the leading jets and is added to its "twin".

31 Mapping functions, Deltas, Delta Widths, Pulls, and Pull Widths for the signal and background data samples where the most probable combination is selected. The blue line is the mapping function used by Karr and Sliwa[7]. . . . . . . . .

32 Mapping functions, Deltas, Delta Widths, Pulls, and Pull Widths for the signal and background data samples where the most probable combination is added to its "twin".

33 Mapping functions, Deltas, Delta Widths, Pulls, and Pull Widths for the signal and background data samples where the most probable combination is selected out of the combinations which contain both of the leading jets. . . . . . . . 7

34 Mapping functions, Deltas, Delta Widths, Pulls, and Pull Widths for the signal and background data samples where the most probable combination is selected out of the combinations which contain both of the leading jets and is added to its "twin". . . . . . . . . . . . . . . . . . . . 72

35 The various correction factors to the RMS by input sample and methodology. . . 74

36 Distributions of corrected widths from the PEs for each of the combination strategies. Upper left is likelihood only, upper right is likelihood added, lower left is 2 leading jets chosen by likelihood, and lower right is 2 leading jets added together. The vertical line and value in each plot is the corresponding statistical error from the measurements. . . . . . . . . . . . . . . . . . . 75

37 Expected statistical errors for each of the four combination strategies based on the width of the PE mass histogram. Upper left is likelihood only, upper right is likelihood added, lower left is 2 leading jets chosen by likelihood, and lower right is 2 leading jets added together. . . . . . . . . . . . . . . 76

38 The $P_{t}, \eta$, and $\phi$ for the jets in both the MC data and the real data. . . . . . . 77

39 The $P_{t}, \eta$, and $\phi$ for the leptons in both the MC data and the real data. . . . . 77 
40 The $P_{t}, \eta$, and $\phi$ for the top quarks in both the MC data and the real data. . . 78

41 The $P_{t}$, rapidity, and $\phi$ for the top-anti-top quark system in both the MC data and the real data. . . . . . . . . . . . . . . . . . . . . . 78

42 The energy, $\phi$, energy in the x-direction, and energy in the y-direction for the $\mathrm{E}_{T}$ in both the MC data and the real data. . . . . . . . . . . . . . . . 79

43 The delta (bias) and pull width from each of the 10 blind samples. . . . . . . . 80

44 Mass mean as a function of the number of interactions per event. . . . . . . . . 101

45 This figure shows the proper decay lengths of the $b$-quark into $\mathrm{J} / \psi K^{+}$. . . . 105

46 Standard set of plots for the signal and background data samples with $b$-tagging where the most probable combination is selected. . . . . . . . . . . 107

47 Standard set of plots for the signal and background data samples with $b$-tagging where the most probable combination is added to its "twin." . . . . . . . 107

48 Standard set of plots for the signal and background data samples with $b$-tagging where the most probable combination is selected out of the combinations which contain both of the leading jets. . . . . . . . . . . . . . . 108

49 Standard set of plots for the signal and background data samples with $b$-tagging where the most probable combination is selected out of the combinations which contain both of the leading jets and is added to its "twin." . . . . . . . . . . 109

50 Top quark mass measurements from the Dalitz-Goldstein method. The red error bars represent the statistical error while the blue error bars represent the statistical and systematic errors added in quadrature. The vertical gray bar shows the interval covered by the combined top quark mass value and errors from all previous measurements at the Tevatron. . . . . . . . . . . . . . . . . 130

51 Top quark mass measurements from the Tevatron. The red error bars represent the statistical error while the blue error bars represent the statistical and systematic errors added in quadrature. The vertical gray bar shows the interval covered by the combined top quark mass value and errors from all previous measurements at the Tevatron. . . . . . . . . . . . . . . . . . 131 


\section{Measurement of the Top Quark Mass in the Di-lepton Channel using the Dalitz-Goldstein Method}




\section{Chapter 1 - Top Quark Physics}

\section{$1.1 \quad$ Introduction}

This dissertation describes a measurement of the top quark mass using the Dalitz-Goldstein method applied to the di-lepton channel on data collected by the Collider Detector Facility at Enrico Fermi National Accelerator Laboratories. The first chapter will give a description of the fundamental theory that drives research in modern Physics. It will be followed by a description of the facilities at Fermi Lab along with details about the experimental apparatus. Chapter 3 will talk about the largest source of systematic errors: the Jets and Jet Energy Corrections. Chapter 4 gives a brief discussion of the di-lepton channel, including its sources of backgrounds. Additionally, it gives a brief discussion of two other methods of measuring the top quark mass in the di-lepton channel. Chapter 5 goes into detail about the Dalitz-Goldstein method and the analysis on which this dissertation is based. The following chapters describe the various sources of systematic errors, the application of $b$-tagging to the di-lepton channel, and finally the results of this analysis.

\subsection{The Fundamental Building Blocks}

The Standard Model is the most widely accepted and experimentally tested theory that describes the composition of matter and how it interacts. There are two major categories of particles that make up the building blocks of the universe: fermions and bosons. The fundamental fermions are split into two families: quarks and leptons. These particles come together to form all of the matter in the universe. Particles such as protons and neutrons are made of quarks, and the electron is an example of a lepton. Bosons are the mediating particles of the interactions in matter. The photon is an example of a boson.

The distinguishing property that separates the fermions from the bosons is spin. All of the fermions have half-integer spin and obey Fermi-Dirac statistics while all of the bosons have integer spins and obey Bose-Einstein statistics.

In the Standard Model, matter interacts with the exchange of gauge bosons. There are four types of interactions, also known as the four forces. They are the Strong Force, Electromagnetic Force, Weak Force, and Gravity. In the Standard Model, each force is mediated by its own type 
of gauge boson(s). Whether or not a particle interacts via one of the four forces depends on its properties. If a particle lacks a certain property, it simply cannot interact with other matter via that force. Conversely, if a particle possesses a certain property, it can and will interact with other matter that also possess this property. The Strong and Weak Forces are known as the nuclear forces and are limited in range. Both have a range of the size of a proton or less. The strong force is what is responsible for binding the protons and neutrons together in the nucleus, and the quarks together in the proton or neutron. It is aptly named as it is the strongest of the four forces and is always attractive. The weak force is responsible for many types of particle decay, including the expected decay modes of the top quark. Its name can be misleading, as it is not the weakest of the four forces; rather, it is just the weaker of the two nuclear forces. The electromagnetic force and gravity are both infinite in range. Outside of the nucleus of an atom, these are the only two forces that are important. The electromagnetic force is responsible for binding electrons to the nucleus of an atom. It is attractive when two particles have opposite signs and repulsive when they are the same. Also, the electromagnetic force is proportional to the magnitude of the two particles' charges, and inversely proportional to the square of the distance between them. It is about two orders of magnitude weaker than the strong force but about four orders of magnitude stronger than the weak force ${ }^{1}$. Gravity is the most familiar of the forces, but by far the weakest. It is about thirty-seven orders of magnitude weaker than the electromagnetic force when compared at the same distance for electrons. It only appears to be strong in everyday life due to the size of astronomical bodies, such as the Earth, and because bulk matter rarely possesses a large net electric charge. It is always attractive, proportional to the masses of the two bodies in question, and inversely proportional to the square of the distance between them. Due to its relative weakness, gravity can be ignored in high energy particle interactions.

The distinguishing property that separates the fundamental fermions into the two families is color. The quarks all possess color and the leptons do not. This means that the only fundamental fermions that interact strongly are the quarks. Gluons also possess color and will interact with both the quarks and each other via the strong force. They are the only bosons

\footnotetext{
${ }^{1}$ It is important to note how forces are being compared. When comparing the EM force to the strong force, its important to state that the comparison is at approximately the size of the proton and that the same types of particles are being compared, up quarks would make a good example in this case. A similar case must be made for the weak force when comparing it to other forces as well.
} 


\begin{tabular}{lccr}
\hline Force & Boson & Symbol & Interactive Property \\
\hline \hline Strong & Gluon & $g$ & color \\
Electromagnetic & Photon & $\gamma$ & electric charge \\
Weak & W and Z & $W^{ \pm} Z^{0}$ & weak charge \\
Gravity & Graviton* & $G$ & mass \\
\hline
\end{tabular}

Table 1: The four forces

that have color, and this fact is what makes Quantum Chromo-Dynamics (QCD) so different from Quantum Electro-Dynamics (QED). The remaining properties, electric charge and weak charge, are possessed by various members of both families of fermions and the gauge bosons. Each family has six members, which are separated into three generations each containing two particles.

\begin{tabular}{cccc}
\hline Generation & I & II & III \\
\hline \hline electric charge & & Quarks & \\
\hline$+\frac{2}{3} \mathrm{e}$ & Up & Charm & Top \\
$-\frac{1}{3} \mathrm{e}$ & Down & Strange & Bottom \\
\hline \hline electric charge & & Leptons & \\
\hline$-\mathrm{e}$ & Electron & Muon & Tau \\
0 & Electron Neutrino & Muon Neutrino & Tau Neutrino \\
\hline
\end{tabular}

Table 2: The three generations of the leptons and quarks

For each particle there exists an antimatter particle, although some particles are their own antiparticles. They have the same masses, but the signs of some of their additive quantum numbers will be switched. For example, the proton $(p)$ and anti-proton $(\bar{p})$ both have a mass of 938.3MeV and a spin of $\frac{1}{2} \hbar$ but the charge of the proton is $+e$ and the charge of the anti-proton is $-e$. When a matter particle comes in contact with its antimatter particle, they will annihilate and produce one or more bosons, if only virtual, whose quantum numbers correspond to those of the particles that annihilated.

\subsection{Leptons}

There are six leptons which easily break down into two groups: charged leptons and neutrinos. All leptons have half-integer spin $\left(\frac{1}{2} \hbar\right)$. The charged leptons all have an electric charge of $-e$ 
$\left(e \approx 0.303(\hbar c)^{\frac{1}{2}}\right)$ and the neutrinos are all neutral. This means that the charged leptons are all affected by the electromagnetic force and the neutrinos are not. The charged leptons also have significant masses, the lightest being the electron and the heaviest being the tau, with the muon falling in the middle. While the electron is stable, the tau and muon both have a finite lifetime and will decay. The discovery of neutrino flavor oscillations indicates that neutrino masses are non-zero, but the inferred masses are so small that for the purposes of this dissertation, they will be treated as massless. All six of the leptons have a property called weak charge which couples them to the weak force and, as stated above, none of them possess color so they do not couple to the strong force. So, the charged leptons all couple to three of the four forces while the neutrinos only interact via the weak force and gravity. From an experimental point of view, this makes the neutrinos extremely difficult to detect and in the case of the Collider Detector Facility (CDF) at FNAL, undetectable. There are three generations of leptons, each containing one charged lepton and its associated neutrino. The first generation contains the electron $\left(e^{-}\right)$ and electron neutrino $\left(\nu_{e}\right)$, the second contains the muon $\left(\mu^{-}\right)$and the muon neutrino $\left(\nu_{\mu}\right)$, and finally the third contains the tau $\left(\tau^{-}\right)$and the tau neutrino $\left(\nu_{\tau}\right)$. Each of the types of neutrinos is associated with its charged lepton through experimentally observed conservation laws.

\begin{tabular}{cccc}
\hline Generation & I & II & III \\
\hline \hline Charge Leptons & $e^{-}$ & $\mu^{-}$ & $\tau^{-}$ \\
mass & $0.5109 \mathrm{MeV}$ & $105.7 \mathrm{MeV}$ & $1.776 \mathrm{GeV}$ \\
lifetime & stable & $2.2 \mu \mathrm{s}$ & $290.6 \mathrm{fs}$ \\
charge & $-e$ & $-e$ & $-e$ \\
\hline Neutrinos & $\nu_{e}$ & $\nu_{\mu}$ & $\nu_{\tau}$ \\
mass & $<2 \mathrm{eV}$ & $<2 \mathrm{eV}$ & $<2 \mathrm{eV}$ \\
lifetime & & & \\
charge & 0 & 0 & 0 \\
\hline
\end{tabular}

Table 3: Properties of the leptons and neutrinos. Be careful not to confuse the symbols $e^{-}$ and $e^{+}$, which stand for the electron and positron, with the charges $-e$ and $+e$ which are $\pm 1.602 \times 10^{-19}$ C. $[1]$

There are also six anti-leptons, the positron $\left(e^{+}\right)$, the mu plus $\left(\mu^{+}\right)$, the tau plus $\left(\tau^{+}\right)$, and the three anti-neutrinos $\left(\overline{\nu_{e}}, \overline{\nu_{\mu}}, \overline{\nu_{\tau}}\right)$. 


\subsection{Quarks}

There are six quarks that, like the leptons, can be separated into two groups by electric charge. Unlike the leptons, none of the quarks are neutral, nor do they have integer values of $e$ charge. Instead, one group consists of quarks with a fractional charge $+\frac{2}{3} e$ and the other with $-\frac{1}{3} e$. Since, just like the leptons, they are fermions, they all also have a spin of $\frac{1}{2} \hbar$. All of the quarks are massive and possess weak charge. In addition, quarks carry a special property called color. Color, in this context, is not the same thing as the concept of color as it would relate to visible light. The property of color is, in essence, strong charge - the property that couples to the strong force. Unlike electric charge, it is neither positive nor negative. The strong charge is called color because it comes in three varieties that, when mixed, combine in a similar way as the colors of visible light. All free particles in the Standard Model are colorless. The three varieties of strong charge are named red, green, and blue (RGB). There are also six anti-quarks which possess anti-color $(\bar{R} \bar{G} \bar{B})$. The six 'flavors' of quarks are Up (u), Down (d), Strange (s), Charm (c), Bottom (b), and Top (t), each of which has an antimatter partner $(\bar{u}, \bar{d}, \bar{s}, \bar{c}, \bar{b}$, and $\bar{t})$.

Together, these twelve particles combine in different ways to form a family of composite particles called hadrons. Hadrons can be identified by their valence quarks, the net combination of quarks of which they are composed. Hadrons come in two groups: baryons and mesons. Baryons (anti-baryons) are composed of three valence quarks (anti-quarks) and mesons are composed of a valence quark and anti-quark pair. Even though all hadrons are made of quarks, which carry color, the hadrons themselves are colorless; the net color charge of any hadron must be 0 . The colors of the quarks in a hadron must add up in a way that is colorless. This postulate stems from the fact that all observed hadrons are colorless and that a single quark has never been observed in nature. For example, the proton is a baryon, which has three valence quarks, each of which must carry a different color. Just like when adding red, green, and blue light gives white light, a red, green, and blue set of quarks will result in a hadron that is colorless. Another example is the $\pi^{+}$meson, which has a d quark and $\bar{d}$ pair of valance quarks. In this case, the color of the quark must be the opposite to the anti-color of the anti-quark: red and anti-red or blue and anti-blue.

Since baryons are made up of three spin $\frac{1}{2}$ quarks, their total spin must be either $\frac{1}{2}$ or $\frac{3}{2}$. 
Therefore baryons are fermions. Two examples are the proton and the neutron. The proton is made up of two $u$ quarks and a d quark and the neutron is made up of one $u$ quark and two d quarks. Also, since mesons consist of two quarks, the total spin must be either 0 or 1 . The latter are known as the vector mesons. Both the proton and the $\pi^{+}$meson have the same charge, but are made up of different quarks. The neutron is neutral, but has two of the same quarks as the proton. This is why the charges of the quarks must be fractional.

Similar to the leptons, not all quarks are stable. The $\mathrm{u}$ and $\mathrm{d}$ quarks are the only stable quarks, the s, c, b, and t quarks all decay quickly. This is why all the stable hadrons are made of $\mathrm{u}$ and $\mathrm{d}$ quarks, while hadrons that are made with one or more of the other quarks must be made in the laboratories.

\begin{tabular}{cccc}
\hline Generation & I & II & III \\
\hline \hline & $u$ & $c$ & $t$ \\
mass & 1.5 to $3.3 \mathrm{MeV}$ & $1.27 \mathrm{GeV}$ & \\
charge & $+\frac{2}{3} e$ & $+\frac{2}{3} e$ & $+\frac{2}{3} e$ \\
\hline & $d$ & $s$ & $b$ \\
mass & 3.5 to $6.0 \mathrm{MeV}$ & $104 \mathrm{MeV}$ & $4.2 \mathrm{GeV}$ \\
charge & $-\frac{1}{3} e$ & $-\frac{1}{3} e$ & $-\frac{1}{3} e$ \\
\hline
\end{tabular}

Table 4: Properties of the quarks[1]

\subsection{Gauge Bosons}

In the Standard Model, there are four types of gauge bosons, one for each of the four types of interactions. These bosons differ from mesons in that they are not composite particles made from the bound state of a quark and anti-quark. Aside from the fact that they have an integer spin, they differ, as elementary particles, from the fundamental fermions in that they are not matter, but instead quanta of their associated gauge fields.

The gauge boson associated with the strong force is called the gluon $(g)$. It is a massless, spin one particle. It couples to the strong force through color, and, as such, possesses color itself. Despite that the gluons are massless, like the photon, the strong force would be infinite in range, similar to the electromagnetic force, were it not for the interferences due to gluons being able to interact with each other. The way in which gluons possess color is not as simple as the 
quarks. There are not simple red, green, and blue gluons. There are eight independent color states of gluons that are represented by linear combinations of pairs of color and anti-color. For example, one such state could be $r \bar{b}+b \bar{r}$.

The photon $(\gamma)$, probably the most commonly known gauge boson, is associated with the electromagnetic force. Photons are massless, colorless, and neutral spin one particles. They do not interact with each other due to their lack of charge. They can be characterized by the energy that they carry, which is related to their frequency. They couple electromagnetically with charged particles.

The weak force has three associated gauge bosons. They are the $Z^{0}, W^{+}$, and $W^{-}$. They are all massive, $M_{z} \approx 91 G e V$ and $M_{w} \approx 80 G e V$, which is why the weak force has such a short range, considerably shorter than that of the strong force. The $W^{+}$and $W^{-}$carry an electric charge of $+e$ and $-e$ respectively. This gives them the ability to interact with each other and other charged particles electromagnetically. Otherwise, they are responsible for the weak force and interact with particles that possess weak charge, including themselves since they also possess weak charge.

The fourth type of boson is the Graviton. The Graviton has yet to be observed and is still theoretical. According to theory, the graviton is massless and has a spin of $2 \hbar$. It gives rise to the force called Gravity, and couples to anything with energy and momentum - which means it couples to everything. According to some higher dimensional theories[8], the graviton may have the special ability to travel into other dimensions. They hypothesize this to help explain why gravity appears to be so much weaker than the other three forces.

\begin{tabular}{ccccc}
\hline Force & Strong & Electromagnetic & Weak & Gravity \\
\hline \hline Boson & Gluon & Photon & IVB & Graviton \\
Symbol & $\mathrm{g}$ & $\gamma$ & $W^{ \pm}, Z^{0}$ & $\mathrm{G}$ \\
Spin & 1 & 1 & 1 & 2 \\
Mass & 0 & 0 & $80 \mathrm{GeV}, 91 \mathrm{GeV}$ & 0 \\
Electric Charge & 0 & 0 & $\pm \mathrm{e}, 0$ & 0 \\
Coupling & Color & Electric Charge & Weak Charge & Energy and Momentum \\
\hline
\end{tabular}

Table 5: Properties of the three gauge bosons and the graviton[1] 


\subsection{The Standard Model}

The Standard Model is a local gauge field theory that describes how all of the known and a few still only theorized particles interact. It is very well supported by experimental data but is incomplete. First of all, it does not include gravity, mainly due to the fact that a quantized field theory of gravity has not yet been developed. Second, the Standard Model contains twentysix arbitrary parameters which include the twelve fermion masses, four quark mixing matrix parameters, four mixing parameters for the neutrinos, three force coupling strengths, two Higgs parameters, and a QCD vacuum angle.

In Physics, both in Classical and Field Theory, it's important to be able to describe the state of a system and its evolution. One of the most powerful formalisms for this is the EulerLagrange Equation. It was originally formalized classically and was eventually generalized to work with Relativity and Field Theory.

$$
\partial_{\mu}\left(\frac{\partial \mathcal{L}}{\partial\left(\partial_{\mu} \phi_{i}\right)}\right)=\frac{\partial \mathcal{L}}{\partial \phi_{i}}
$$

where $\phi_{i}$ is the field coordinate. At the heart of this equation is the Lagrangian, $\mathcal{L}$. Once the proper Lagrangian can be written down for a system, everything that needs to be known about that system can be derived from it. In the case of Particle Physics, the Lagrangian tells the story of how particles and fields interact with each other. An example is the Dirac Lagrangian.

$$
\mathcal{L}=i \hbar c \bar{\psi} \gamma^{\mu} \partial_{\mu} \psi-m c^{2} \bar{\psi} \psi
$$

$\psi$ describe a spinor field, particle of spin $\frac{1}{2}$ and mass $m$, with an adjoint field $\bar{\psi}$. This Lagrangian describes a free fermion and consists of a kinetic term and a mass term.

As stated above, the Standard Model is a local gauge field theory, which means that it is invariant under local gauge transformations. $\psi$ is a four component spinor. Each of its four components is, in general, complex; however $\bar{\psi} \psi$ is completely real and describes things that can be observed in reality. If a transformation is applied to $\psi$ such that $\psi \rightarrow e^{i \theta} \psi$, where $\theta$ is the phase angle and a real number, the Lagrangian will be unchanged. This type of transformation is called a Global Gauge Transformation, and if the Lagrangian remains unchanged, it exhibits global gauge invariance. However, if $\theta$ is a function of position, $\theta(x)$, then the transformation $\psi \rightarrow e^{i \theta(x)} \psi$ is a Local Gauge Transformation. Eq. 2 is not invariant 
under local gauge transformations. However, if an additional term is added, $-\left(q \bar{\psi} \gamma^{\mu} \psi\right) A_{\mu}$, the Lagrangian becomes

$$
\mathcal{L}=i \hbar c \bar{\psi} \gamma^{\mu} \partial_{\mu} \psi-m c^{2} \bar{\psi} \psi-\left(q \bar{\psi} \gamma^{\mu} \psi\right) A_{\mu}
$$

and local gauge invariance is achieved. $A_{\mu}$ describes a new field, called a gauge field, which transforms like,$A_{\mu} \rightarrow A_{\mu}-\partial_{\mu} \lambda(x) . \lambda(x) \equiv-\frac{\hbar c}{q} \theta(x)$ and $q$ is the associated charge of the particles. The new term in the Lagrangian describes how a spinor, $\psi$, interacts with a gauge field, $A_{\mu}$. To make the Lagrangian complete, "free" terms have to be added for the gauge field. The Proca Lagrangian describes a spin 1 massive vector field,

$$
\mathcal{L}=-\frac{1}{16 \pi} F^{\mu \nu} F_{\mu \nu}+\frac{1}{8 \pi}\left(\frac{m_{A} c}{\hbar}\right) A^{\nu} A_{\nu}
$$

where $F^{\mu \nu}=\partial^{\mu} A^{\nu}-\partial^{\nu} A^{\mu}$ and $m_{A}$ is the mass of the vector field. The first term in this Lagrangian works out just fine, but the second term has a problem: it is not locally gauge invariant. However, if the mass of the gauge field $\left(A_{\mu}\right)$ is zero, then the second term will vanish and the Lagrangian will be

$$
\mathcal{L}=i \hbar c \bar{\psi} \gamma^{\mu} \partial_{\mu} \psi-m c^{2} \bar{\psi} \psi-\left(q \bar{\psi} \gamma^{\mu} \psi\right) A_{\mu}-\frac{1}{16 \pi} F^{\mu \nu} F_{\mu \nu}
$$

It is obvious now that $A_{\mu}$ is the electromagnetic potential and the Lagrangian describes how Dirac fields interact with the electromagnetic field. Or, in particle terms, it describes how fermion particles interact with the photon, a massless gauge boson.

The real phase angle $\theta$ in the gauge transformation described above can be thought of as a $1 \times 1$ matrix. These matrices are unitary; the product of their hermitian adjoint with themselves is one. The group of all such unitary matrices is called $U(1)$, and the symmetry is called $U(1)$ gauge invariance. In a similar manner, Quantum Chromodynamics gives rise to a very similar Lagrangian; however it has some important differences. Since each flavor of quark can come in three different colors, $\psi$ will now have three components, one for each color. Also, the local gauge transformation can be represented by a $3 \times 3$ matrix which belongs to the $S U(3)$ group. Therefore, the Lagrangian in this case will need to have $S U(3)$ gauge invariance. This group can be expressed in a singlet and an octet representation. Once again, massless field vectors will appear, but this time there will be eight of them. They are the eight gluons introduced in section 1.4. The Lagrangian for QCD will look like this.

$$
\mathcal{L}=i \hbar c \bar{\psi} \gamma^{\mu} \partial_{\mu} \psi-m c^{2} \bar{\psi} \psi-\left(q \bar{\psi} \gamma^{\mu} \lambda \psi\right) \cdot \mathbf{A}_{\mu}-\frac{1}{16 \pi} \mathbf{F}^{\mu \nu} \cdot \mathbf{F}_{\mu \nu}
$$


$\mathbf{A}_{\mu}$ are the eight gluon fields and the $\lambda$ are the Gell-Mann matrices. $\mathbf{F}_{\mu \nu}$ is defined as

$$
\mathbf{F}_{\mu \nu} \equiv \partial^{\mu} \mathbf{A}^{\nu}-\partial^{\nu} \mathbf{A}^{\mu}-\frac{2 q}{\hbar c}\left(\mathbf{A}^{\mu} \times \mathbf{A}^{\nu}\right)
$$

In both QCD and QED the gauge fields that appear in the Lagrangian from requiring local gauge invariance are massless, as a result the mass term in the "free" Lagrangian for those fields vanishes. However, the gauge fields that are associated with the Weak Force are not massless; therefore, the mass term will not vanish; it cannot simply be written into the Lagrangian since it will not preserve local gauge invariance.

In 1961, Glashow began to formulate a theory where the electromagnetic and weak forces were not separate entities, but instead two manifestations of the same unified force. In 1967, Weinberg and Salam helped formalize this theory into what is know as Electroweak Theory. In this theory, only left handed particles ${ }^{2}$ couple with the weak gauge fields. The property that couples to the weak force is called weak isospin. Each generation of left-handed leptons form an isotopic doublet, and each right-handed lepton forms its own isotopic singlet. The left- and right-handed leptons transform in the following way:

$$
\begin{array}{r}
\psi_{L} \rightarrow e^{i \vec{\alpha}(x) \cdot \mathbf{T}} e^{i \beta(x) \frac{Y}{2}} \psi_{L} \\
\psi_{R} \rightarrow e^{i \beta(x) \frac{Y}{2}} \psi_{R}
\end{array}
$$

Both $\alpha(x)$ and $\beta(x)$ are arbitrary real functions. $\mathbf{T}=\tau^{+} \hat{x}+\tau^{-} \hat{y}+\tau^{3} \hat{z}, \tau^{ \pm}=\frac{1}{2}\left(\tau^{1} \pm i \tau^{2}\right)$, and the $\tau^{1,2,3}$ are the Pauli matrices. $Y$ is the Weak Hyper charge and is related to the electric charge, $Q$, and the third component of isospin, $I^{3} \cdot Q=I^{3}+\frac{1}{2} Y$. These transformations form a group of $2 \times 2$ matrices which belong to $S U(2)$ and $1 \times 1$ matrices which belong to $U(1)$. This gauge theory has an $S U_{L}(2) \times U_{Y}(1)$ symmetry, where the subscripts suggest that is applies to left-handed particle and particles that have hyper-charge.

The Lagrangian will contain the following terms for the fermions and gauge fields:

\footnotetext{
${ }^{2}$ The notion of handedness in chirality and helicity are connected, but not equivalent. Helicity is defined as the projection of a particle's spin onto its direction of momentum. If a particle is traveling in the same direction as its spin is pointing, then it would have a helicity of +1 and would be referred to as "Right-handed". If the direction of motion and spin are anti-aligned, its helicity is -1 and referred to as "Left-handed". However, in terms of how particles interact weakly, handedness refers to chirality. In GWS theory, particles form eigen states of chirality, not helicity. Only in the special case of massless particle do chirality and helicity become the same.
} 
$\bar{\psi}_{L} \gamma^{\mu}\left(i \hbar c \partial_{\mu}-\frac{1}{2} g \mathbf{T} \cdot \mathbf{W}_{\mu}-\frac{1}{2} g^{\prime} Y B_{\mu}\right) \psi_{L}+\bar{\psi}_{R} \gamma^{\mu}\left(i \hbar c \partial_{\mu}-\frac{1}{2} g^{\prime} Y B_{\mu}\right) \psi_{R}-\frac{1}{16 \pi} \mathbf{W}^{\mu \nu} \cdot \mathbf{W}_{\mu \nu}-\frac{1}{16 \pi} B^{\mu \nu} B_{\mu \nu}$

Here $\mathbf{W}_{\mu}=W_{\mu}^{+} \hat{x}+W_{\mu}^{-} \hat{y}+W_{\mu}^{3} \hat{z} . W_{\mu}^{ \pm}$are the wave functions representing the charged intermediate vector bosons, $W^{ \pm}$. This accounts for the two charged gauge bosons in this theory, but there are still two more neutral bosons: $Z_{\mu}^{0}$, the third massive weak guage boson, and $A_{\mu}$, the photon. They are related to the third component of $\mathbf{W}_{\mu}$ and $B_{\mu}$.

$$
\begin{gathered}
A_{\mu}=B_{\mu} \cos \theta_{w}+W_{\mu}^{3} \sin \theta_{w} \\
Z_{\mu}^{0}=-B_{\mu} \sin \theta_{w}+W_{\mu}^{3} \cos \theta_{w}
\end{gathered}
$$

$\theta_{w}$ is called the weak mixing angle and is a free parameter of the theory. Its experimental value is approximately $30^{\circ}$. At this point, the electro-weak Lagrangian has many terms, none of which resemble mass terms similar to the ones in the QED and QCD Lagrangians. It appears that all of the particles involved, including the fermions, are massless.

In order to account for the masses of the weak gauge bosons and the fermions involved in this theory, a scalar field is introduced into the Lagrangian and a technique called "Spontaneous Symmetry Breaking" is employed. This scalar field is also an isotopic doublet. The Lagrangian contains these terms for the new scalar fields:

$$
\left|\left(i \hbar c \partial_{\mu}-\frac{1}{2} g \mathbf{T} \cdot \mathbf{W}_{\mu}-\frac{1}{2} g^{\prime} Y B_{\mu}\right) \phi\right|^{2}+\frac{1}{2} \mu^{2} \phi^{*} \phi-\frac{1}{4} \lambda^{2}\left(\phi^{*} \phi\right)^{2}
$$

Both $\lambda$ and $\mu$ are real constants. The potential associated with this new scalar field has multiple minima, which correspond to multiple ground states. If an expansion is done about one of the minima with a change of variables, the Lagrangian will transform and new terms will arise. One of these terms will be a mass term for this new scalar field, and there will also be a few leftover terms involving a massless scalar boson. These bosons are known as Goldstone Bosons and are undesired. However, with a clever choice of a gauge, the undesired bosons will be transformed away. All that will be left is a Lagrangian with mass terms for the appropriate leptons and gauge bosons that were in the theory from the start, and a new massive scalar boson known as the Higgs Boson.

To see how the masses of the $W^{ \pm}$and $Z^{0}$ arise, simply apply this spontaneous symmetry breaking technique to the first term in Eq. 13. Two mass terms will arise, one for the $Z^{0}$ 
and one for the $W^{ \pm} \mathrm{S}$. Using $\bar{g}=\sqrt{g^{2}+g^{\prime 2}}, g / \bar{g}=\cos \theta_{w}$, and $g^{\prime} / \bar{g}=\sin \theta_{w}$, along with the constants in the mass terms, this simple relation between the masses of the $W^{ \pm}$and $Z^{0}$ arises:

$$
\frac{m_{W}}{m_{Z}}=\cos \theta_{w}
$$

In addition to the terms in Eq. 10 and Eq. 13, the Lagrangian will contain terms involving $\phi$, the left-handed doublets, and the right handed singlets for the fermions, both for quarks and leptons. Using the same technique as above will give rise to mass terms for all of the fermions.

It is important to note that unlike the leptons, the left handed weak doublets for the quarks are not simply each generation of quarks. The weak eigenstates are not the same as the mass eigenstates. Instead, there is mixing between generations with the weak force eigenstates. The left handed weak doublets are:

$$
\left(\begin{array}{c}
u \\
d^{\prime}
\end{array}\right)_{L},\left(\begin{array}{c}
c \\
s^{\prime}
\end{array}\right)_{L},\left(\begin{array}{c}
t \\
b^{\prime}
\end{array}\right)_{L}
$$

where

$$
\left(\begin{array}{c}
d^{\prime} \\
s^{\prime} \\
b^{\prime}
\end{array}\right)=\left(\begin{array}{lll}
V_{u d} & V_{u s} & V_{u b} \\
V_{c d} & V_{c s} & V_{c b} \\
V_{t d} & V_{t s} & V_{t b}
\end{array}\right)\left(\begin{array}{c}
d \\
s \\
b
\end{array}\right)
$$

This matrix is called the Cabibbo Kobayashi Maskawa (CKM) quark mixing matrix. The values in this matrix are related to the likelihood that a quark will decay into another lighter quark via a weak process. For example, the probability that a top quark will decay into a bottom quark is proportional to $\left|V_{t b}\right|^{2}$. If the CKM-matrix was diagonal, then there would be no mixing of quarks between generations and weak decays between generations of quarks should not be observed experimentally. However, the CKM-matrix is not diagonal and this is well supported by experiment. The magnitude of the values of the CKM-matrix are[1]:

$$
\left(\begin{array}{ccc}
\left|V_{u d}\right| & \left|V_{u s}\right| & \left|V_{u b}\right| \\
\left|V_{c d}\right| & \left|V_{c s}\right| & \left|V_{c b}\right| \\
\left|V_{t d}\right| & \left|V_{t s}\right| & \left|V_{t b}\right|
\end{array}\right)=\left(\begin{array}{ccc}
0.97419 \pm 0.00022 & 0.2257 \pm 0.0010 & 0.00359 \pm 0.00016 \\
0.2256 \pm 0.0010 & 0.097334 \pm 0.00023 & 0.0415_{-0.0010}^{+0.0010} \\
0.00874_{-0.00037}^{+0.00026} & 0.0407 \pm 0.0010 & 0.999133_{-0.000043}^{+0.000044}
\end{array}\right)
$$




\subsection{The Top Quark}

After the discovery of the bottom quark in 1977, and the measurement of its weak isospin, $T_{3}=-\frac{1}{2}$, physicists began searching for its bigger brother, the top quark. Until the early 1990s, these searches increased the lower limits of its mass. In April of 1994, the top quark was discovered by CDF in proton-anti-proton collisions in the Tevatron. The top quark completed the third generation isospin doublet with $T_{3}=+\frac{1}{2}$. It has a spin of $\frac{1}{2} \hbar$ and an electric charge of $+\frac{2}{3} e$.

The Tevatron produces top quarks and anti-top quarks by colliding protons and anti-protons at a center of momentum energy $\sqrt{s}=1.96 \mathrm{GeV}$. Top quarks are produced by quark-anti-quark annihilation or gluon-gluon fusion, with the former responsible for over $85 \%$ of $t \bar{t}$ events. After the collision, if the gluon produced in the annihilation has sufficient energy, it can decay into a top and anti-top quark pair. As can be seen in the CKM matrix, the top quark almost exclusively decays into a bottom quark and a $W^{+}$boson. Likewise, the anti-top quark's favored mode of decay is into an anti-bottom quark and a $W^{-}$boson. Both the bottom and anti-bottom quarks hadronize and then the b-hadrons decay in the detector and produce a jet. The $W^{ \pm}$ bosons can decay hadronically into a $q \bar{q}$ pair which will produce a pair of jets. They can also decay leptonically, producing a charged lepton and associated neutrino.

Since $t$ and $\bar{t}$ are always produced in a pair, the final state can consist of all hadronic, lepton plus jets, and di-lepton events. Table 6 displays all of the different decay modes of $t \bar{t}$ events and their branching ratios where $q \bar{q}^{\prime}$ signifies any quark-anti-quark pair except for $t \bar{t}$.

All-hadronic $t \bar{t}$ events result in six energetic jets: two from the bottom quarks and four from the decays of the $W^{ \pm}$bosons. Given the kinematic parameters of the six jets and knowledge of which were produced by the bottom quarks it is possible to reconstruct the mass of the top quark. This channel has a branching ratio of approximately $44 \%$, the largest of the three. While this channel will produce the largest number of $t \bar{t}$ events, which makes it attractive statistically, it also has the largest background of the three channels. It is plagued by higher order QCD background processes that also produce multiple jets.

In the lepton plus jets channel, only one of the $W^{ \pm}$decays hadronically while the other decays leptonically. Like the all-hadronic channel, there are six decay products: one charged lepton and one neutrino, which escapes detection, from the $W$ that decayed leptonically, two 


\begin{tabular}{lcc}
\hline Decay Mode & Branching Ratio & Decay Channel \\
\hline \hline$t \bar{t} \rightarrow\left(q \bar{q}^{\prime} b\right)\left(q \bar{q}^{\prime} \bar{b}\right)$ & $36 / 81$ & All Hadronic \\
\hline$t \bar{t} \rightarrow\left(q \bar{q}^{\prime} b\right)\left(e^{-} \bar{\nu}_{e} b\right)$ or $\left(e^{+} \nu_{e} b\right)\left(q \bar{q}^{\prime} b\right)$ & $12 / 81$ & Lepton + Jets \\
$t \bar{t} \rightarrow\left(q \bar{q}^{\prime} b\right)\left(\mu^{-} \bar{\nu}_{\mu} \bar{b}\right)$ or $\left(\mu^{+} \nu_{\mu} b\right)\left(q \bar{q}^{\prime} b\right)$ & $12 / 81$ & \\
$t \bar{t} \rightarrow\left(q \bar{q}^{\prime} b\right)\left(\tau^{-} \bar{\nu}_{\tau} \bar{b}\right)$ or $\left(\tau^{+} \nu_{\tau} b\right)\left(q \bar{q}^{\prime} b\right)$ & $12 / 81$ & \\
\hline$t \bar{t} \rightarrow\left(e^{+} \nu_{e} b\right)\left(\mu^{-} \bar{\nu}_{\mu} b\right)$ or $\left(\mu^{+} \nu_{\mu} b\right)\left(e^{-} \bar{\nu}_{e} b\right)$ & $2 / 81$ & \\
$t \bar{t} \rightarrow\left(e^{+} \nu_{e} b\right)\left(\tau^{-} \bar{\nu}_{\tau} \bar{b}\right)$ or $\left(\tau^{+} \nu_{\tau} b\right)\left(e^{-} \bar{\nu}_{e} \bar{b}\right)$ & $2 / 81$ & \\
$t \bar{t} \rightarrow\left(\mu^{+} \nu_{\mu} b\right)\left(\tau^{-} \bar{\nu}_{\tau} \bar{b}\right)$ or $\left(\tau^{+} \nu_{\tau} b\right)\left(\mu^{-} \bar{\nu}_{\mu} \bar{b}\right)$ & $2 / 81$ & \\
$t \bar{t} \rightarrow\left(e^{+} \nu_{e} b\right)\left(e^{-} \bar{\nu}_{e} \bar{b}\right)$ & $1 / 81$ & \\
$t \bar{t} \rightarrow\left(\mu^{+} \nu_{\mu} b\right)\left(\mu^{-} \bar{\nu}_{\mu} \bar{b}\right)$ & $1 / 81$ & \\
$t \bar{t} \rightarrow\left(\tau^{+} \nu_{\tau} b\right)\left(\tau^{-} \bar{\nu}_{\tau} \bar{b}\right)$ & $1 / 81$ & \\
\hline
\end{tabular}

Table 6: Various decay modes of $t \bar{t}$ events

jets from the $W$ that decayed hadronically, and two $b$-jets from the $t \bar{t}$ decay. The signature for this channel is therefore a lepton plus four or more jets. Even though the neutrino escapes detection, the mass of the top quark can be kinematically reconstructed if the mass of the neutrino is assumed to be zero. The branching ratio for this channel is also approximately 44\%; however, events involving the short lived $\tau$ lepton are typically not included, which drops the observable branching ratio to $30 \%$. This channel is also contaminated by events involving higher order QCD processes, though the requirement of an energetically charged lepton makes the QCD background a smaller set than the all-hadronic channel; however, this channel also includes a new set of backgrounds involving the direct production of $W^{ \pm}$bosons.

The di-lepton channel contains events where both of the $W^{ \pm}$decayed leptonically. Similar to the lepton plus jets channel, events involving the $\tau$ lepton are not included, which drops the effective branching ratio from approximately $11 \%$ to $5 \%$. In addition to having a small branching ratio, this channel has the disadvantage of having two undetectable neutrinos. The signature of the channel is therefore two highly energetic charged leptons and two energetic jets. The major advantage of this channel is that its two charged leptons help to distinguish it from many other type of multi-jet events and therefore it has the lowest background of the three channels.

In this dissertation, the di-lepton channel is used exclusively. Figures 1 and 2 show two tree-level Feynman diagrams of the dominate process leading to the production and decay of a $t \bar{t}$ event in the di-lepton channel. As was mentioned in the beginning of Section 1.6, there 
are twenty-six arbitrary parameters in the SM. This analysis is a measurement of one of those parameters. Aside from being one of the 26 parameters, the top quark mass, together with the mass of the $\mathrm{W}$ boson, can help give indirect information on the mass of the higgs boson predicted by the SM (Figure 3). The radiative corrections in the mass of the $\mathrm{W}$ boson contain terms that are quadratically dependent on the mass of the top quark and logarithmically dependent on the mass of the higgs boson. Therefore, knowing both the $\mathrm{W}$ and top masses precisely can put limits on the mass of the higgs boson. Additionally, a low top quark mass may indicate physics that goes beyond the SM. A low mass would be consistent with the Minimal Super-symmetric Standard Model (MSSM), which predicts the existence of two higgs doublets (Figure 4).

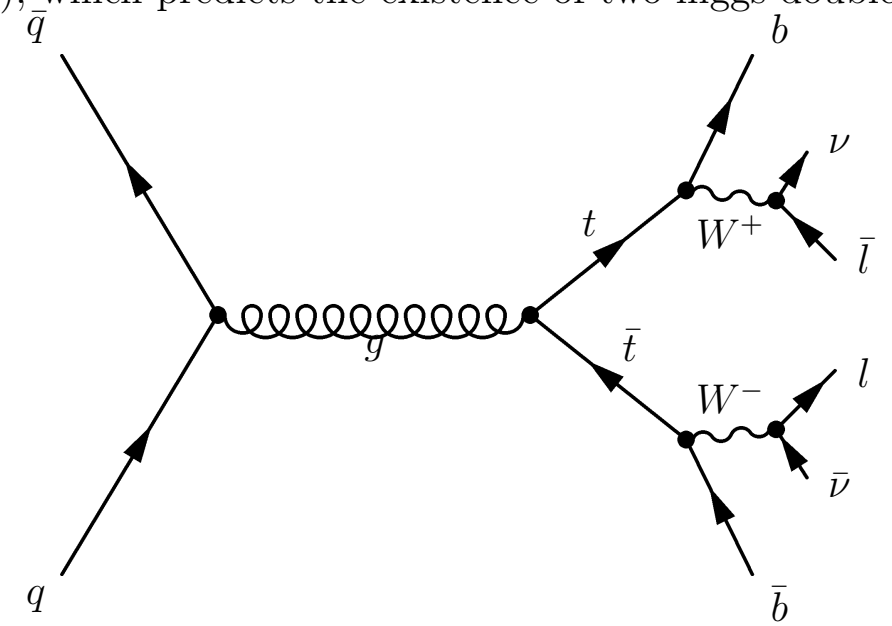

Figure 1: Feynman diagram illustrating $t \bar{t}$ production via $q \bar{q}$ annihilation in the di-lepton channel.

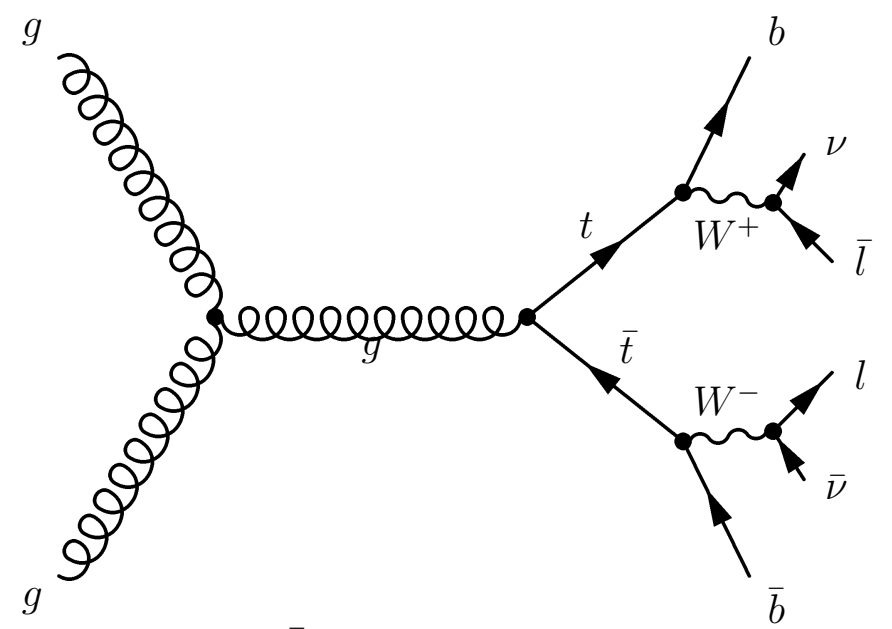

Figure 2: Feynman diagram illustrating $t \bar{t}$ production via gluon fusion in the di-lepton channel. 


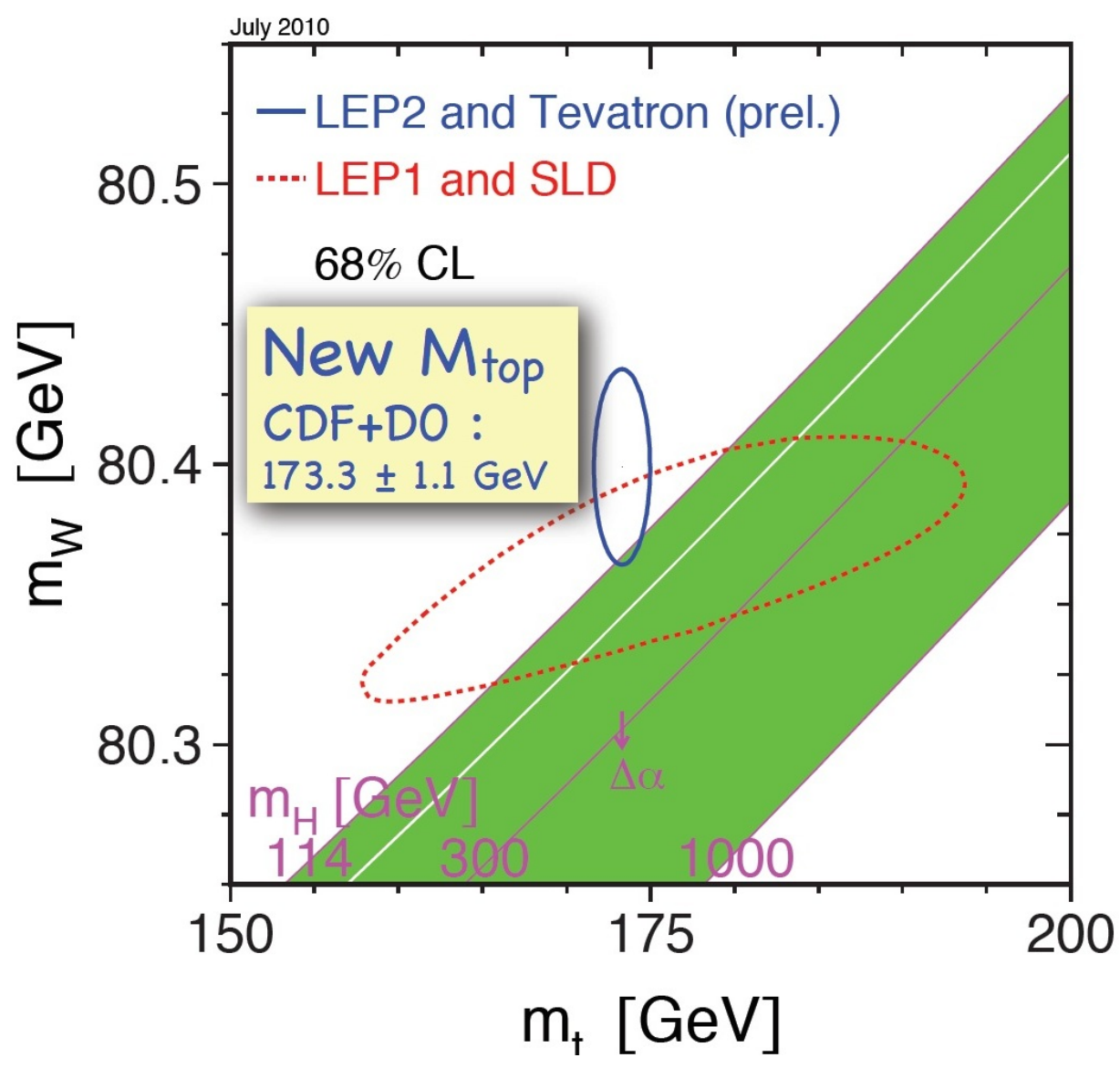

Figure 3: The green band in this plot shows the allowed range of masses for the higgs boson given a range of top and $\mathrm{W}$ boson masses. The blue oval shows the allowed region given measurements of the top and W masses from LEP2 and the Tevatron. As more precise measurements of the $\mathrm{W}$ and top masses are made, the overlap between the green band and the blue oval may change and possibly disappear all together.[4] 


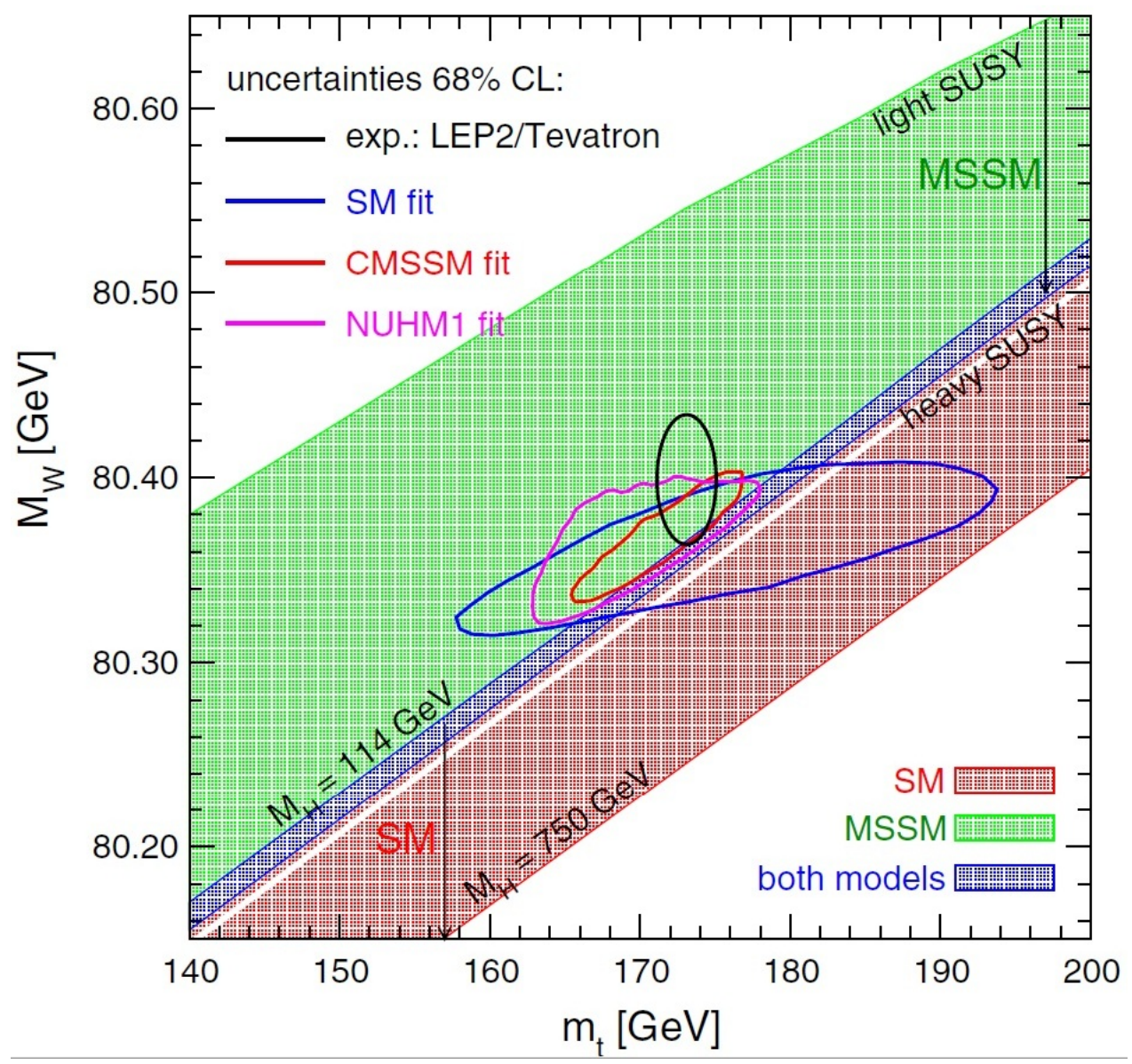

Figure 4: Similar to the previous plot, this shows the regions where the current measured values of the top and $\mathrm{W}$ masses agree with modern theories that allow for the existence of the higgs boson. It is clear that a light top mass favors the MSSM model.[5] 


\section{Chapter 2 - The Experimental Apparatus}

\section{$2.1 \quad$ FNAL and CDF}

There are two major collider experiments at FNAL: D $\varnothing$, named after its position in the Tevatron, and CDF, short for Collider Detector at Fermilab. Both experiments are large, multipurpose detectors designed to measure and probe much of the same physics. This chapter will describe in some detail the Tevatron and the CDF detector, from which all the data for this dissertation was collected.

\subsection{The Tevatron}

The Tevatron is the hadron collider located in Batavia, IL at Fermi National Accelerator Laboratories. It collides beams of protons and anti-protons at a center-of-momentum energy of 1.96 $\mathrm{GeV}$. It is capable of producing most of the particles described in the standard model including the top quark.

The beams begin in the Cockcroft-Walton generator, which negatively ionizes hydrogen gas, and exposes the ions to an electric field to accelerate them up to $750 \mathrm{keV}$. Next, the ionized gas is passed into a $150 \mathrm{~m}$ long linear accelerator called the Linac. There, it is accelerated up to $400 \mathrm{MeV}$. At this point, the hydrogen is passed through a carbon foil, which strips away the electrons, leaving only the nuclei (protons) to be sent into the Booster. The Booster is a small ring accelerator that accelerates the protons up to $8 \mathrm{GeV}$. Once this energy is reached, the protons are transfered to the Main Injector. The Main Injector has many functions, one of which is to accelerate the protons up to an energy of $150 \mathrm{GeV}$ and inject them into the Tevatron. Another job of the Main Injector is to accelerate protons up to $120 \mathrm{GeV}$ for antiproton production. This is done by sending protons to a nickel target. Colliding protons with a nickel target will produce many types of secondary particles, some of which will be anti-protons. The anti-protons are sent into a storage ring where they are accumulated and cooled through a process called stochastic cooling. After sufficient anti-protons are collected, they are sent to the Recycler where they undergo additional cooling, and are stored in preparation for injection into the Tevatron. Each time the production of protons or anti-protons is completed, a bunch is created. The process is repeated thirty-six times to create thirty-six proton bunches and 


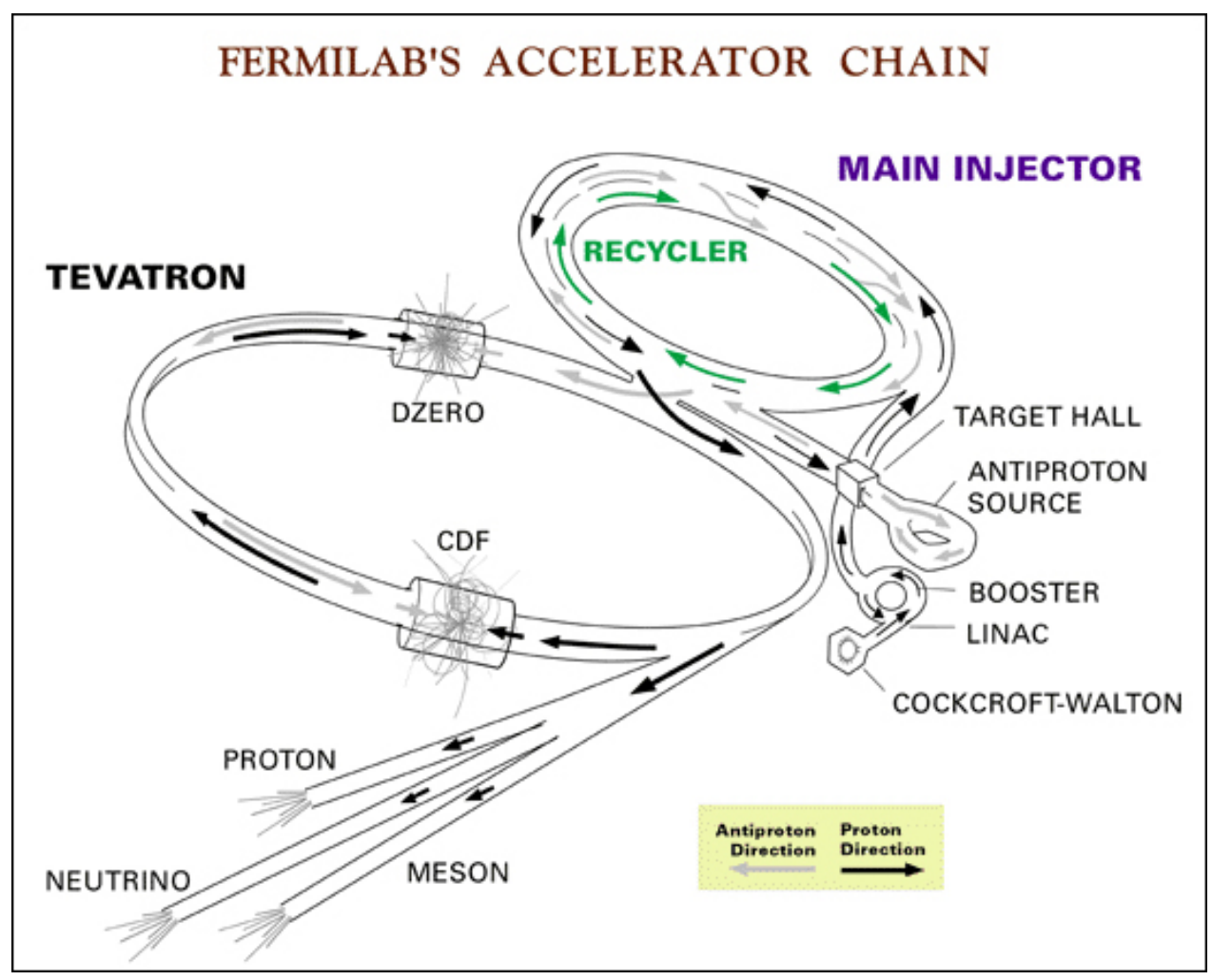

Figure 5: Graphical representation of proton and anti-proton beams at Fermilab[6]

thirty-six anti-proton bunches. Once all of the bunches are created, they are loaded into the Tevatron. The proton and anti-proton beams travel in the same beam pipe, but in opposite directions. After the Tevatron is fully loaded, the beams are accelerated up to an energy of $980 \mathrm{GeV}$. The beams are then collided by low-beta quadrupole magnets which bring the beams into sharp focus at two positions in the Tevatron, D0 and B0, the positions of the DØ and CDF detectors respectively.

Producing anti-protons is a difficult process. The luminosity $(L)$ of the Tevatron depends on both the number of protons $\left(N_{1}\right)$ in the beam and the number of anti-protons $\left(N_{2}\right)$, as well as the cross section of the beams $(A)$, number of bunches $(n)$, and the revolution frequency $(f)$.

$$
L=f n \frac{N_{1} N_{2}}{A}
$$

The number of anti-protons is typically the limiting factor. In order to keep a high luminosity, instead of sending the anti-proton beam to a beam dump at the end of a store, it is decelerated and sent back to the Recycler where it joins newly produced anti-protons. Typical beam 


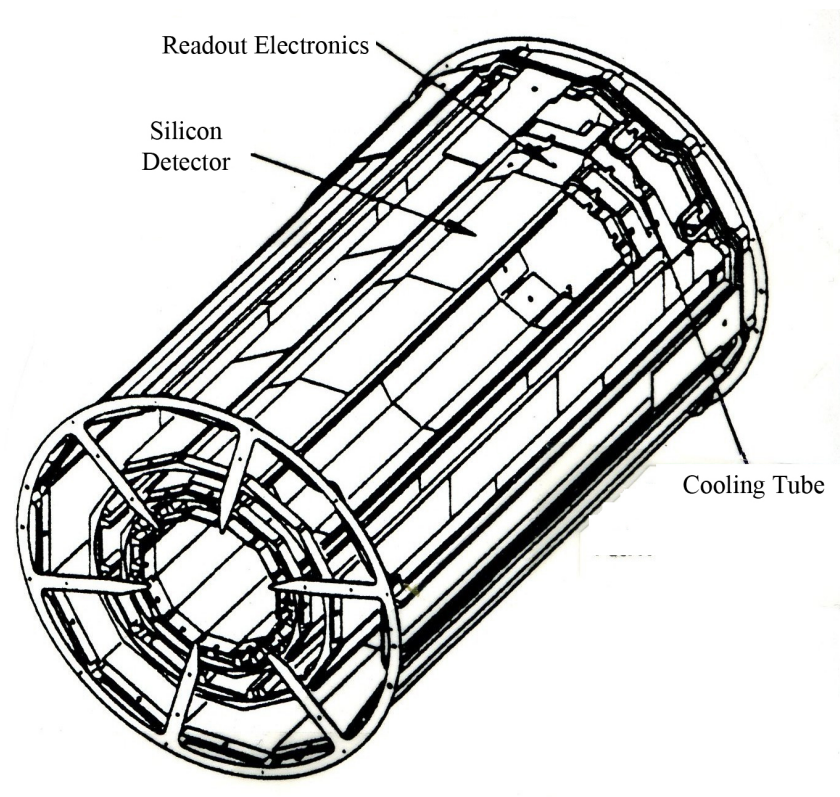

Figure 6: One barrel of the SVXII[6]

intensities are $6 \times 10^{12}$ particles for the proton beam and $1.3 \times 10^{12}$ particles for the anti-proton beams.

\subsection{The Collider Detector at Fermilab}

The detector is comprised of many subsystems, each of which is its own type of particle detector. At the center of the detector is the silicon vertex detector (SVXII). The purpose of the SVXII is to provide accurate detection of multiple vertices in each event. It is composed of three cylindrical barrels of double sided silicon microstrip detectors. Each barrel has five layers, with each layer made up of twelve "ladders". The "ladders" are the low mass substrates that support the silicon crystals. The SVXII is $96 \mathrm{~cm}$ long.

Outside of the SVXII are the intermediate silicon layers (ISL). The ISL has 5 layers, one for the central region and two for each plug region. Similar to the SVXII, the layers of the ISL are organized into "ladders", except that instead of two crystals per ladder, the ISL has three. Together, the SVXII and ISL cover a range of $|\eta| \leq 2.0$ and provide standalone tracking and $b$-tagging in that region.

The Central Outer Tracker (COT) is the third part of CDF's tracking system. While it is 


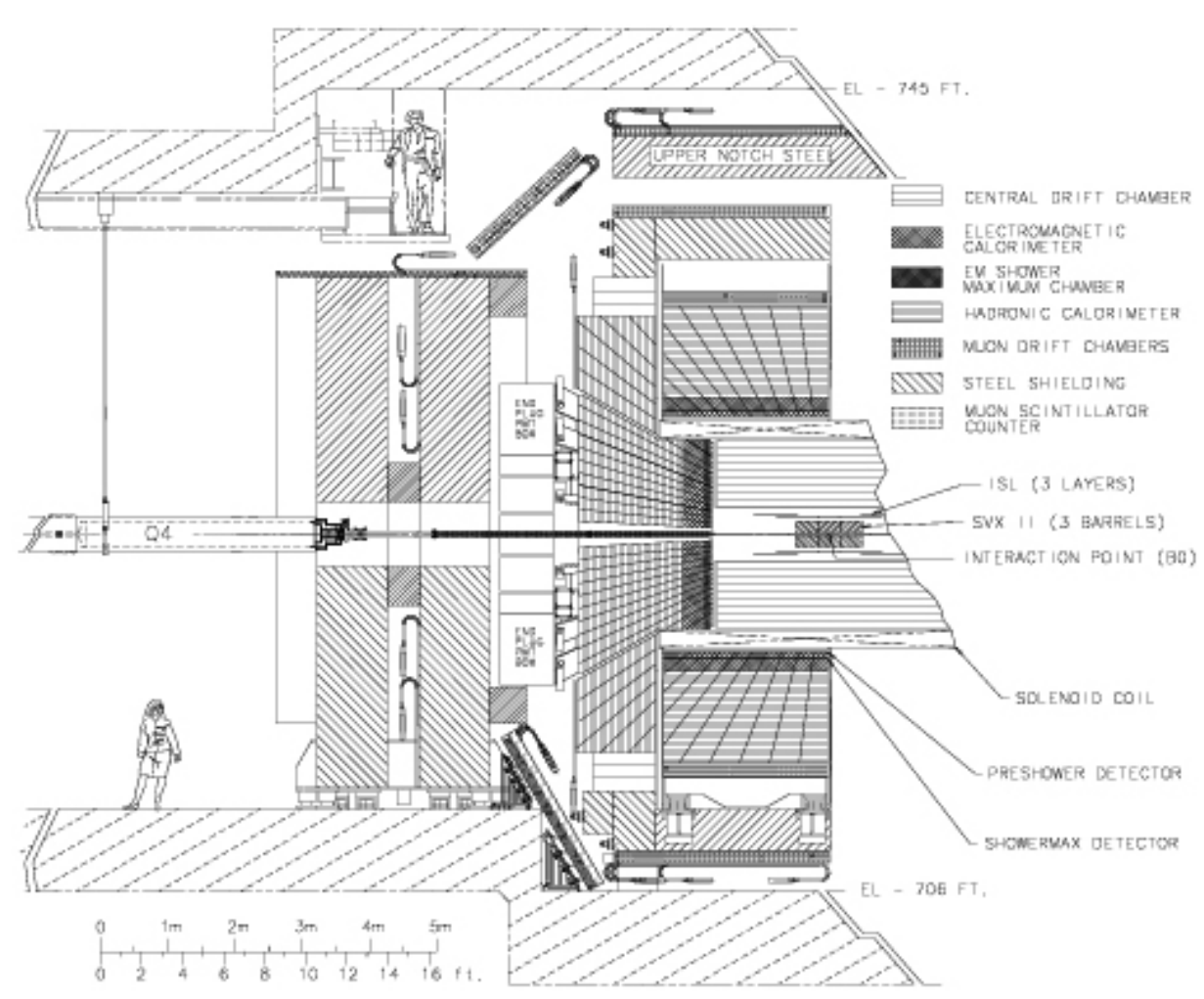

Figure 7: Cross-section of the collider detector at Fermilab[6]

not located at the very center, the heart of the detector is the COT. It is an open cell drift chamber. The COT and the silicon detectors are surrounded by a superconducting solenoid that produces a $1.4 \mathrm{~T}$ magnetic field parallel to the beam axis. It provides tracking of particles in the region $|\eta| \leq 1.0$. The COT is broken down into four axial and four stereo superlayers. The number of cells in each superlayer depends on the radius of the superlayer, the innermost having one hundred sixty-eight cells and the outermost having four hundred eighty. Each cell has twelve sense wires. As charged particles pass though the COT, the gas inside is ionized and begins to drift toward the sense wires. After a charged particle has passed through many cells, its path can be tracked.

Outside of the tracking systems are the calorimeters. There are two types of calorimeters in the detector: the inner is the electromagnetic (EM) calorimeter and the outer is the hadronic calorimeter. The EM calorimeter is made of twenty-three layers of lead and scintillator. Each layer is composed of $4.5 \mathrm{~mm}$ lead and $4 \mathrm{~mm}$ scintillator. There is an embedded two-dimensional readout strip chamber at shower maximum. The EM calorimeter is read out with photomul- 
tiplier tubes. The hadronic calorimeter also has twenty-three layers, but is made of iron and scintillator. The iron in each layer is $50 \mathrm{~mm}$ thick, and the scintillator is $6 \mathrm{~mm}$ thick. Together, the central and plug calorimeters cover a range of $|\eta| \leq 3.0$. The calorimetry is segmented into towers that radiate outward from the optimal collision point.

The outer part of the detector is the muon detection system. There are four parts to this system: the Central Muon System (CMU), the Central Muon Upgrade (CMP), the Central Muon Extension (CMX), and the Intermediate Muon System (IMU). Each of these systems is a combination of drift tube detectors and scintillator detectors, except for the CMU which is only a drift tube detector. The CMU and CMP cover the same range, $|\eta| \leq 0.6$, while the CMX covers $0.6 \leq|\eta| \leq 1.0$ and the IMU covers $1.0 \leq|\eta| \leq 1.5$.

\subsection{Event Triggering}

Given the large number of channels, cells, layers, wires, and PMTs that make up the detector, there is a enormous amount of data from each event. This is further compounded by the 7.6 $\mathrm{MHz}$ crossing rate of the Tevatron. This massive data rate is made manageable through the CDF triggering system. There are three levels of triggering that help decide which events have interesting physics in them, and if so move their data to permanent storage.

The Level 1 trigger is a storage pipeline that can store the data for forty-two beam crossings. The Level 1 decision is made on the calorimeter, central tracking chamber, and the muon detector data. The Level 1 trigger has $4 \mu s$ to make a decision on an event as it moves through the pipeline, and is done synchronously with the data in the pipeline. If an event is deemed interesting, it is passed to the Level 2 buffer. The acceptance rate for the Level 1 trigger is designed to be less than $50 \mathrm{kHz}$.

There are four Level 2 buffers. Once an event is loaded into a buffer from the Level 1 accept, that buffer can not be used again until the Level 2 decision is made. If all four of the buffers become filled, then any new events accepted by the Level 1 trigger will be lost and the experiment will incur deadtime. The data from the silicon tracking system, the central tracking chamber, the calorimetry, and the muon detectors is used to make the Level 2 decision. Level 2 has an average decision time of $20 \mu \mathrm{s}$ and has an acceptance rate of about $300 \mathrm{~Hz}$. Together Level 1 and Level 2 accept about one in twenty-thousand events. 
Once an event has been accepted by the Level 2 trigger, it is passed to the DAQ buffers, where it waits to be sent to the Level 3 computer farm via a network switch. Each event has all of its data assembled for analysis. After it has been analyzed and accepted, it is written to permanent storage. The Level 3 acceptance rate is about $75 \mathrm{~Hz}$. [6] 


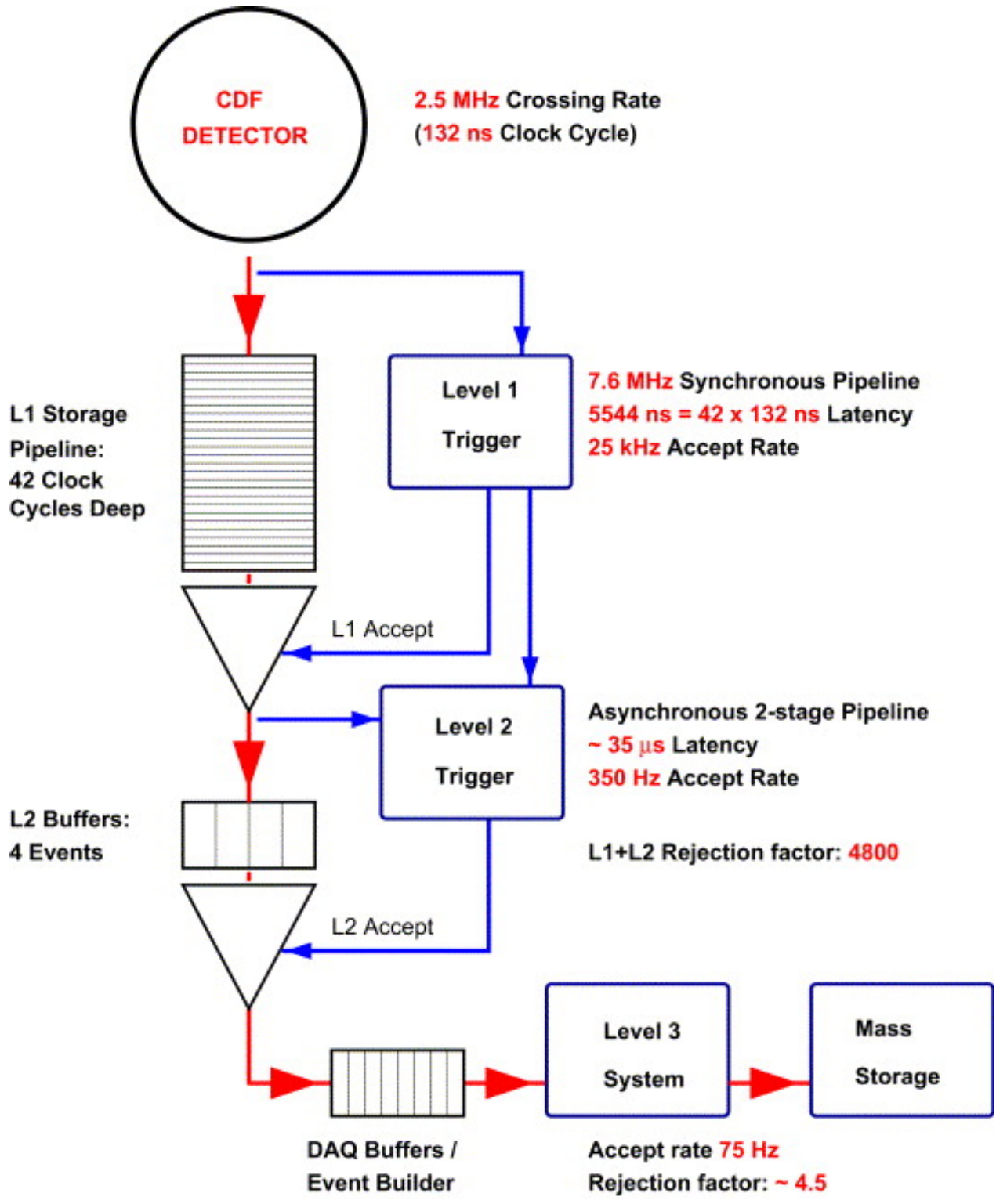

Figure 8: Block diagram of the CDF Run II trigger system[6] 


\section{Chapter 3 - The Jets}

\subsection{Introduction}

As it will be shown in chapter 6 , the uncertainty in jets' energy measurement is the largest source of systematic error for this analysis. This uncertainty arises from two sources: the detector itself and physics of jets. The detector's ability to measure energies is limited by the composition of materials used in the calorimeters, for example, the details of construction like thickness of absorber and scintillator layers. Additionally, any neutrinos produced in a jet will escape the detector without depositing any energy. Jet energies detected via electromagnetic(EM) showers (photons, electrons, $\pi^{0}$ ) are measured better in electromagnetic calorimeters than those interacting strongly, as the EM showers have a larger, by orders of magnitude, number of particles than in hadronic showers. The error in measuring the energy of a jet goes as $1 / \sqrt{n}$, where $n$ is the number of particles that make up the jet. This chapter describes how the jets are reconstructed and how their energies are corrected.

\subsection{Jet Reconstruction}

To reconstruct jets in the detector, a cone-based algorithm is used. It begins by selecting preclusters made of towers in the calorimeter with a transverse energy $\left(E_{T}\right)$ greater than 1.0 $\mathrm{GeV}$, where the highest tower is located near the center of the cluster. Each precluster is limited in size with a maximum $\phi$ of $105^{\circ}$ and maximum $\eta$ of 0.7 units. These limits correspond to a maximum cluster size of $7 \times 7$ towers in the central calorimeter and $21 \times 7$ towers in the forward and plug regions. Clusters are made by finding the $E_{T}$ weighted centroid of the preclusters forming cones in the $\phi-\eta$ plane with a fixed radius of $R=\sqrt{(\Delta \phi)^{2}+(\Delta \eta)^{2}}$. If a tower is inside the radius of the cone and has an $E_{T}>100 \mathrm{MeV}$, it is included in the cluster. Then the $E_{T}$ weighted centroid of the new cluster is recalculated and this process is repeated until the tower assignment to all the clusters remains constant. If overlap of towers occurs between clusters, further redistribution is required. It has been found through Dijet studies that the optimal cone size is in the rage $0.4<R<1.0$. A cone size of 0.4 is used in this analysis and is also the most common choice throughout the experiment. 


\subsection{Standard Jet Energy Corrections}

The jet energy as measured by the detector's calorimetry needs to be corrected due to a number of detector and physics effects. Historically, there have been eight levels of corrections which, depending on the analysis, will be employed in combination. This analysis uses levels one, four, and five to correct the jet energies. Levels two, three, and eight have become obsolete and are no longer used. Levels six and seven are only used if a particular analysis calls for them.

\subsubsection{Level 1 Relative Energy Correction}

The relative energy correction is also known as the $\eta$-dependent correction. It corrects for the non-linearity of the energy response in the calorimeters. It is the first correction that needs to be done and makes the detector's response as a function of $\eta$ uniform. In a $2 \rightarrow 2$ process the $E_{T}$ of the jets should be equal. This is used to scale the jets outside the $0.2<|\eta|<0.6$ region to the jets inside this region. The region corresponds to the central calorimeter away from the cracks, which is the best understood calorimeter in the detector.

\subsubsection{Level 2 Central Calorimeter Stability}

As the detector ages, the energy response of the calorimeters changes. This correction is dependent on the time period in which the data was taken, and is applied on a run by run basis. This level is no longer in use and its correction is taken into account in the energy scale calibration.

\subsubsection{Level 3 Scale Correction}

The level 3 correction is a scale factor that relates the old Run I absolute energy corrections to the current ones, and allows the old absolute corrections to be used. It is important to verify that the linearity of the detector has not changed since Run I. An ADC-to-GeV scale factor is downloaded to the hardware on a tower by tower basis and used online in the L1 and L2 triggers. The same scale factor is used in L3 and offline. Next, the CEM and CHA scales have to be checked so that an offline correction can be applied if needed. To set the CEM scale factor $Z^{0} \rightarrow e^{+} e^{-}$are reconstructed so that the $Z^{0}$ mass is 


$$
Z^{0}=91.19 \pm 0.002 G e V
$$

The scale factors need to be updated periodically as the CEM ages.

The CHA energy scale is set with the minimum ionizing particle (MIP) peak of low and high $P_{T}$ muons. The jet energy scale is check by using photon-jet balancing, since the CEM can measure photon energy very precisely. Essentially, once the scales for the CEM and CHA are set, photon-jet energies from RUN II can be compared to Run I and should be identical.

This level is also no longer used since the experiment has a new Run II absolute energy correction, making this scale factor obsolete.

\subsubsection{Level 4 Multiple Interactions Correction}

Given the current luminosity of the proton and anti-proton beams and their thirty-six bunches, it is likely that an event will overlap with an additional minimum bias event. This is where there is more than one collision in the same beam crossing. Energy from particles in this extra event may fall in the cone of the jets from the primary event. This energy needs to be subtracted from the cone before analysis. The correction is parameterized in terms of additional energy per number of vertices in the event. Efficiency and fake rate of the vertex reconstruction algorithm are different than in Run I and therefore the additional energy per vertex is different[2]. Additionally, this correction is assigned an uncertainty of $100 \mathrm{MeV} /$ vertex.

Table 7 shows the additional energy per vertex that was calculated in both Run I and Run II.

\begin{tabular}{lccc}
\hline GeV & Cone 0.4 & Cone 0.7 & Cone 1.0 \\
\hline \hline Run I & 0.297 & 0.910 & 1.858 \\
Run II & 0.260 & 0.781 & 1.581 \\
\hline
\end{tabular}

Table 7: Additional energy per vertex by cone size.[2]

\subsubsection{Level 5 Absolute Energy Correction}

After correcting for any non-linear response from the calorimeter in the un-instrumented regions, the jet energies may still be incorrect. For historical reasons, a $50 \mathrm{GeV}$ pion was used as a 
calibration point. The energy deposited by this pion may not be measured to $50 \mathrm{GeV}$, so a correction in the data is required, which is the calibration. This correction is called the Absolute Energy Correction. It depends on the jet fragmentation properties, the non-linearity of the calorimeter, the difference in calorimeter response to $\pi^{0}$ and $\pi^{ \pm}$, and the cone size used for the jets.

The corrections are piecewise quadratic functions derived empirically with a break-point at 100GeV. Eq. 17 and Table 8 describe the correction.

$$
f_{a b s}=\left(P_{0}+P_{1} \cdot P_{T}+P_{2} \cdot P_{T}^{2}\right) / 100
$$

\begin{tabular}{l|c|c|c|c|c|c}
\hline$P_{T}$ & \multicolumn{3}{|c|}{$\leq 100.0$} & \multicolumn{3}{c}{$>100.0$} \\
\hline conesize & 0.4 & 0.7 & 1.0 & 0.4 & 0.7 & 1.0 \\
\hline \hline$P_{0}$ & 1.37 & 2.09 & 3.29 & -4.03 & -1.49 & -0.37 \\
$P_{1}$ & 1.1946 & 1.227 & 1.2229 & 1.1794 & 1.197 & 1.20704 \\
$P_{2}$ & -0.0008742 & -0.000839 & -0.0007133 & -0.0001805 & -0.000182 & -0.0001878 \\
\hline
\end{tabular}

Table 8: Parameterization of the Absolute Energy Correction[2]

\subsubsection{Level 6 Underlying Event Subtraction}

Given the current luminosity from the collisions of the proton anti-proton beams, it is likely that an event will have more than one interaction. Energy from particles in these extra interactions may fall in the cone of the jets from the primary interaction. This energy needs to be subtracted from the cone before analysis. This correction can be dependent on the process under study. However, it is assumed that the spectator interactions are well modeled by minimum bias events and taken care of in the level 4 correction. If additional precision is required an additional correction can be applied and is shown in Table 9. This analysis does not use this correction nor level seven and eight; however, these corrections are needed for the systematic studies which will be described in Chapter 6 . 


\begin{tabular}{lccc}
\hline GeV & Cone 0.4 & Cone 0.7 & Cone 1.0 \\
\hline \hline Run I & 0.65 & 1.98 & 4.05 \\
Run II & 0.52 & 1.56 & 3.14 \\
\hline
\end{tabular}

Table 9: Underlying Event Subtraction energy by cone size.[2]

\subsubsection{Level 7 Out-of-Cone Correction}

The energy from the original parton that decays into a jet may not be entirely contained in the jet cone. Daughter particles that are part of the jet may lie outside the cone radius. This correction is intended to match the jet energy to the original parton energy by accounting for energy from particles that are Out-of-Cone. This correction is independent of detector performance and entirely relies on the parton fragmentation functions. The correction is parameterized by the following

$$
P_{T}(O C)=A[\text { conesize }] *\left(1.0-B[\text { conesize }] * e^{-C[\text { conesize }] * p t}\right)
$$

where

\begin{tabular}{lccc}
\hline conesize & A & B & C \\
\hline \hline 0.4 & 22.999 & 0.915 & 0.00740 \\
0.7 & 8.382 & 0.846 & 0.00728 \\
1.0 & 3.227 & 0.832 & 0.00817 \\
\hline
\end{tabular}

Table 10: Out-of-Cone Correction parameterization[2]

\subsubsection{Level 8 Splash Out Correction}

The final correction takes into account energy deposited in the calorimeter outside of a cone radius of $\mathrm{R}=1.3$. The correction is $0.25 \mathrm{GeV}$. This level is also no longer used. The out-of-cone definition has been expanded to take in this correction. 


\section{Chapter 4 - The Di-lepton Channel}

\subsection{Introduction}

This chapter begins with a brief explanation of two other methods for measuring the top quark mass. It then goes on to explain how di-lepton events are selected in the data and ends with a description of the types of backgrounds expected to contaminate the signal in the di-lepton channel.

\subsection{Top Mass Measurements in the Di-lepton Channel}

Due to the fact that di-lepton events have two neutrinos that escape the detector unseen, the problem of solving for the top mass becomes under-constrained. There are two more unknowns than the number of constraints if only the kinematic equations are used. To overcome this problem and still be able to take advantage of the Di-lepton Channel's low background, a number of creative solutions have been devised[9][10]. Two of these solutions will be discussed here.

\subsubsection{The Template Method}

To overcome the constraint problem, the template method makes a few assumptions. The mass of the neutrinos are assumed to be zero, the masses of the $\mathrm{W}$ bosons are $80.4 \mathrm{GeV}$, and the mass of the top quark is assumed to be equal to the mass of the anti-top quark. Additionally, information from the missing $E_{t}$ is used. This is nearly enough to solve the problem with 23 equations and 24 variables. To fully constrain the problem, the template method introduces the equation $P_{t \bar{t}}=P_{t_{z}}+P_{\bar{t}_{z}}$. This equation expresses that the z-component of the $t \bar{t}$ system is equal to the sum of the z-components of the top and anti-top quarks, this ignores the possible transverse momentum of the $t \bar{t}$ system.

Since the measured quantities, such as the lepton momenta, jet energies, and missing $E_{t}$ have experimental uncertainties, the template method uses a smearing technique with the jet energies, missing $E_{t}$, and $P_{t \bar{t}}$. This method randomly generates these values for an event based on the measured value and expected error. After repeating this a large number of times and kinematically reconstructing the event, this method produces a distribution of top masses. 
Each event can produce four possible solutions: two from the two undetected neutrinos, and two from the possible ways to pair the jets with the leptons. If an event has more than two jets, only the jets with the highest $E_{t}$ are used; therefore, an event can produce four different top mass distributions. To choose between the four distributions, the method uses the following criteria. When comparing two solutions that are produced from the two allowed kinematic possibilities, the one that has a smaller effective mass of the $t \bar{t}$ system is preferred. Not every combination of randomly generated values will result in a solution. Therefore, each of the four distributions will have a different number of entries. The probability of the distribution will be greater if it has more entries, when compared to the other solutions. When comparing two solutions that result from the two possible ways that the jets and leptons can be paired, the distribution with the higher probability is selected. In the end, this criteria will result in only one of the four distributions being selected.

The last step in the template method is to apply a likelihood fit to the real data events. Using a variety of Monte Carlo(MC) generated top events with different $M_{t}$, Monte Carlo templates are created. These MC templates are used to help create a function that relates the reconstructed mass to the original true mass of the event. Similarly, templates for different types of background are created, and together with the signal templates, a maximum likelihood method is applied to the real data events to calculate the mass of the top quark.

\subsubsection{The Matrix Element Method}

Another method for measuring the top quark mass is the Matrix element method. Given the measured quantities from an event, $\mathbf{x}$, there is a conditional probability, $P\left(\mathbf{x} \mid M_{t}\right)$, that relates to the top mass, $M_{t}$. That probability is known from theoretical calculation, based on our theoretical understanding of the top quark production process in the SM:

$$
P\left(\mathbf{x} \mid M_{t}\right)=\frac{1}{\sigma\left(M_{t}\right)} \frac{d \sigma\left(M_{t}\right)}{d \mathbf{x}}
$$

$\frac{d \sigma}{d \mathbf{x}}$ is the per-event differential cross-section. If the momentum of each of the partons involved in the interaction could be deduced from the final state particles, it would be simple to calculate $\frac{d \sigma}{d \mathbf{x}}$. Instead, it is necessary to integrate over quantities like the neutrino and $b$-quark momenta, which are either not measured or not directly measured. Special transfer functions are used 
to relate the measured jet energies to the $b$-quark energies. These are typically expressed as $f(p, j)$, which is the probability of measuring jet energy $j$ given parton energy $p$. The total expression for the probability can be written:

$$
P\left(\mathbf{x} \mid M_{t}\right)=\frac{1}{N} \int d \Phi_{6}\left|\mathcal{M}_{t \bar{t}}\left(p ; M_{t}\right)\right|^{2} \prod_{j e t s} f\left(p_{i}, j_{i}\right) f_{P D F}\left(q_{1}\right) f_{P D F}\left(q_{2}\right)
$$

In this expression, $N$ is a normalization factor, the integral is over the phase space of the particles, the product is only over the leading two jets, $q_{i}$ are the vectors for the incoming partons, and $p$ is a vector of the resulting parton quantities. $\left|\mathcal{M}_{t \bar{t}}\left(p ; M_{t}\right)\right|^{2}$ are the matrix elements for top-anti-top production, and how this method gets its name.

The probability will yield a distribution in $M_{t}$, like in the Template Method; however, unlike the Template Method, each of the probability distributions for the real data events are multiplied together to form a joint likelihood. The expectation value from this joint likelihood is taken to be the measured mass of the top quark for this method.

\subsection{Event Selection in the Di-lepton Channel}

Lepton candidates are split into two groups, electron candidates and muon candidates. Both of these groups have many sub-types which will be defined in the following sections. Each type will either be classified as tight or loose. In order for an event to be defined as a Di-lepton event, it must have a minimum of two lepton-type objects, at least one of which must be classified as tight. It must also have two jet candidates, both of which must be classified as tight. In addition to the lepton and jet requirements, there are also event level requirements.

\subsubsection{Electron Selection Cuts}

There are three types of electron candidates of interest to this analysis; central electromagnetic (CEM), phoenix (PHX), and non-isolated central electromagnetic (NICEM). The first two are classified as tight while the NICEM is loose.

CEM and NICEM objects must have a track in the COT that extrapolates to a cluster in the

central electromagnetic calorimeter. Additionally, the cluster cannot be near the boundaries of the calorimeter, where energy measurements are unreliable. The PHX objects have to lie in a 
fiducial region of the detector as well, but differ from CEM and NICEM objects in that their clusters are found in the plug calorimeters. Electron candidates that result from the conversion of a photon to an $e^{+} e^{-}$pair before reaching the COT are rejected.

Each of these three types of electron candidates have specific cuts. The CEM object variables that are used for the cuts are as follows:

1. $E_{t}$ : The transverse electromagnetic energy is the EM energy of the cluster in the CEM calorimeter multiplied by but the sine of the polar angle from the best COT track pointing to the cluster.

2. Track $P_{t}$ : The transverse momentum of the COT track measured with the track curvature in the COT in the Magnetic Field.

3. $E_{\text {had }} / E_{E M}$ : The ratio of the hadronic calorimeter energy to the EM calorimeter energy.

4. E/P: The ratio of the EM cluster transverse energy to the COT transverse momentum.

5. $Q *|\Delta x|$ : The charge extrapolated from the direction curvature of the COT track multiplied by the distance in the $r-\phi$ plane between the COT track and the matching CES cluster.

6. $|\Delta z|$ : The distance in the r-z plane between the COT track and the matching CES cluster.

7. $\chi_{\text {strip }}^{2}$ : A comparison of the CES shower profile in the r-z view (strip view) with that of profiles from test beam electrons.

8. $L_{s h r}$ : A comparison of the lateral shower profile of the calorimeter cluster and that of the test beam.

9. $\left|Z_{0}\right|$ : The $\mathrm{z}$ intersection of the COT track with the beam axis in the r-z plane.

10. COT Track Quality: The associated electron track must pass through at least 3 axial and at least 2 segments with at least 5 hits per segment.

11. Isolation: The sum $E_{t}$, excluding the electron $E_{t}$, in all of the EM calorimeter towers inside a cone of radius $R=\sqrt{(\delta \phi)^{2}+(\delta \eta)^{2}}=0.4$ centered about the electron cluster and divided by the electron cluster $E_{t}$. 
The NICEM cuts are all the same as the CEM cuts with this exception of the isolation cut, which is not required.

The PHX electron candidate variables used in the cuts are:

1. (1) $E_{t}$

2. (2) $\mid$ Pes2dEta $\mid: \eta$ of the matching 2d PES cluster used to determine the fiduciality of the electron candidate.

3. (3) $E_{\text {had }} / E_{E M}$

4. (4) Pem $3 \times 3$ FitTow and Pem $3 \times 3$ Chisq: The number of towers used by the $3 \times 3$ PEM cluster fitter and the associated $\chi^{2}$ of the fit.

5. (5) Pes $2 d 5 \times 9 U$ and Pes $2 d 5 \times 9 \mathrm{~V}$ : The $5 / 9$ shower profile ratio of the $\mathrm{u}$ and $\mathrm{v}$-strips of the best matching $2 \mathrm{~d}$ PES cluster.

6. (6) $\Delta R$ : The matching distance in the transverse plane between the Pem $3 \times 3$ Fit coordinates and the 2D PES coordinates of the PEM cluster

7. (7) $\left|\operatorname{PhxTrk} Z_{0}\right|$ : The $\mathrm{z}$ intersection of the phoenix track with the beam axis in the $\mathrm{r}-\mathrm{z}$ plane.

8. (8) PhxMatch: Variable that tells if the Phoenix track is a match with the PEM cluster.

9. (9) PhxTrkSiHits: Number of hits in the phoenix detector

10. (10) Isolation

\subsubsection{Muon Selection Cuts}

There are many types of muon candidates, which are defined by the part of the muon detection system they passed through. There are two types that are classified as tight (CMUP and CMX) and eight types that are classified as loose (CMU, CMP, CMIO, NICMUP, NICMX, NICMU, NICMP, and RHOCMX). All of the types that begin with NI are the non-isolated versions of their matching counterparts, and must pass all of the same selection cuts with the exception 


\begin{tabular}{|c|c|c|c|}
\hline Cut & CEM & NICEM & PHX \\
\hline$E_{t}$ & $>20.0 \mathrm{GeV}$ & $>20.0 \mathrm{GeV}$ & $>20.0 \mathrm{GeV}$ \\
\hline Track $P_{t}$ & $>10.0 \mathrm{GeV}$ & $>10.0 \mathrm{GeV}$ & $>10.0 \mathrm{GeV}$ \\
\hline$E_{\text {had }} / E_{E M}$ & $<0.055+0.00045^{*} E \mathrm{GeV}$ & $<0.055+0.00045^{*} E \mathrm{GeV}$ & $<0.05$ \\
\hline$E / P$ & $<2.0 \| \operatorname{TrkPt}>50.0 \mathrm{GeV}$ & $<2.0 \|$ TrkPt $>50.0 \mathrm{GeV}$ & - \\
\hline$Q *|\Delta x|$ & $>-3.0 \mathrm{~cm} \mathrm{\&}<1.5 \mathrm{~cm}$ & $>-3.0 \mathrm{~cm} \mathrm{\&}<1.5 \mathrm{~cm}$ & - \\
\hline$|\Delta z|$ & $<3.0 \mathrm{~cm}$ & $<3.0 \mathrm{~cm}$ & - \\
\hline$\chi_{\text {strip }}^{2}$ & $<10.0$ & $<10.0$ & - \\
\hline$L_{s h r}$ & $<2.0$ & $<2.0$ & - \\
\hline$z_{0}$ & $<60.0 \mathrm{~cm}$ & $<60.0 \mathrm{~cm}$ & $<60.0 \mathrm{~cm}$ \\
\hline TrkAxSeg & $\geq 3$ & $\geq 3$ & - \\
\hline TrkStSeg & $\geq 2$ & $\geq 2$ & - \\
\hline Isolation & $<0.1$ & $\geq 0.1$ & $<0.1$ \\
\hline $\mid$ Pes2dEta $\mid$ & - & - & $>1.2 \&<2.0$ \\
\hline Pem3x3FitTow & - & - & $\neq 0$ \\
\hline Pem3x3Chisq & - & - & $<10$ \\
\hline Pes5x9U & - & - & $>0.65$ \\
\hline Pes5x9V & - & - & $>0.65$ \\
\hline$\Delta R$ & - & - & $<3.0 \mathrm{~cm}$ \\
\hline PhxMatch & - & - & TRUE \\
\hline$N_{\text {hits }}^{S i}$ & - & - & $\geq 3$ \\
\hline
\end{tabular}

Table 11: Electron selection cuts for CDF di-lepton events

of the isolation cut. Both the Central Muon System and Central Muon Upgrade cover the same range in pseudo-rapidity, $|\eta|<0.6$, so a muon that passes through one system will pass through the other, and should leave hits in both systems. If this happens, those muons are called CMUP muons. Muons that leave hits in only one of the two systems are named after that system: CMU for the Central Muon System and CMP for the Central Muon Upgrade. The Central Muon Extension covers a range in pseudo-rapidity of $0.6<|\eta|<1.0$. Muons that pass through this detector and leave hits are called the CMX muons. The RHOCMX muons are the same as CMX muons except that they have a COT exit radius (Rho) less than $140 \mathrm{~cm}$. All of the preceding types of muons must have an associated track in the COT. If there is a track in the COT that points to a cluster in the calorimeter that is consistent with a minimum ionizing particle and there is no hit in the muon detectors, it is identified as a CMIO muon. Muons of this type are also referred to as stubless. This covers the non-fiducial regions of the 
detector and extends the range for muon search in pseudo-rapidity to $|\eta| \leq 1.2$. Muons that pass through either of the Barrel Muon Upgrade Detectors and leave hits are the BMU and NIBMU muons, and they are not used in di-lepton channel analyses.

In addition to the fiducial cuts, a muon is required to pass additional cuts based on these variables:

1. $P_{t}$ : The corrected transverse momentum of the COT track measured with the track curvature in the COT in the Magnetic Field.

2. E $E_{\text {had }}$ : Hadronic Calorimeter Energy

3. $E_{E M}$ : Electromagnetic Calorimeter Energy

4. COT Track Quality

5. $\left|D_{0}\right|$ : The Impact parameter of the associated track corrected for beam position.

6. $\left|Z_{0}\right|$

7. Isolation

Each of the stubbed muons may be required to pass additional cuts that are specific to the detector they passed through. Variables related to those cuts are:

8. $\left|\Delta x_{C M U}\right|:$ The distance in $r-\phi$ plane between the extrapolated track and the track segment in the CMU.

9. $\left|\Delta x_{C M P}\right|$ : The distance in $r-\phi$ plane between the extrapolated track and the track segment in the CMP.

10. $\left|\Delta x_{C M X}\right|$ : The distance in $r-\phi$ plane between the extrapolated track and the track segment in the CMX.

CMIO Muons have a cut based on the additional variable:

11. $E_{E M}+E_{\text {had }}$ : Sum of the energies from the hadronic and electromagnetic calorimeters.

CMX an RHOCMX Muons have a cut based on the additional variable:

12. Rho: COT exit radius. 


\begin{tabular}{|c|c|c|c|c|c|c|c|c|}
\hline Cut & units & CMUP & CMX & CMU & CMP & RHOCMX & CMIO & NI-type \\
\hline corrPt & $\mathrm{GeV}$ & \multicolumn{7}{|c|}{$\geq 20.0$} \\
\hline$E_{\text {had }}$ & $\mathrm{GeV}$ & \multicolumn{7}{|c|}{$<6.0+\operatorname{Max}(0.0,0.0280 *(P-100.0))$} \\
\hline$E_{E M}$ & $\mathrm{GeV}$ & \multicolumn{7}{|c|}{$<2.0+\operatorname{Max}\left(0.0,0.0115^{*}(P-100.0)\right)$} \\
\hline TrkAxSeg & & \multicolumn{7}{|c|}{$\geq 3$} \\
\hline TrkStSeg & & \multirow{3}{*}{\multicolumn{7}{|c|}{$\begin{array}{c}\geq 2 \\
\text { if } \#_{\text {hits }}^{S i}=0,<0.2 ; \text { if } \#_{\text {hits }}^{S i}>0,<0.02 \\
<60.0\end{array}$}} \\
\hline$\left|D_{0}\right|$ & $\mathrm{cm}$ & & & & & & & \\
\hline$\left|Z_{0}\right|$ & $\mathrm{cm}$ & & & & & & & \\
\hline Isolation & & $<0.1$ & $<0.1$ & $<0.1$ & $<0.1$ & $<0.1$ & $<0.1$ & $\geq 0.1$ \\
\hline$\left|\Delta x_{C M U}\right|$ & $\mathrm{cm}$ & $\leq 3.0$ & - & $\leq 3.0$ & . & - & - & $\leq 3.0$ \\
\hline$\left|\Delta x_{C M P}\right|$ & $\mathrm{cm}$ & $\leq 5.0$ & - & - & $\leq 5.0$ & - & - & $\leq 5.0$ \\
\hline$\left|\Delta x_{C M X}\right|$ & $\mathrm{cm}$ & - & $\leq 6.0$ & - & - & $\leq 6.0$ & - & $\leq 6.0$ \\
\hline$E_{E M}+E_{\text {had }}$ & $\mathrm{GeV}$ & . & - & - & - & - & $>0.1$ & \\
\hline Rho & $\mathrm{cm}$ & - & $>140$ & - & - & $\leq 140$ & - & $>140$ \\
\hline
\end{tabular}

Table 12: Muon selection cuts for CDF di-lepton events

\subsubsection{Jet Selection Cuts}

In order for an event to be considered a di-lepton event, it must have at least two tight jets. Unlike the leptons, only tight jets are considered in the di-lepton selection, and all loose jets are ignored. For a jet to be classified as tight, it must pass the following cuts:

1. Corrected $E_{t}>15 \mathrm{GeV}$

2. $|\eta|<2.5$

\subsubsection{Event Selection Cuts}

Beyond the lepton and jet requirement, there are more cuts that need to be made on the event level before an event can be called a di-lepton event. These cuts are:

1. $\mathrm{E}_{T}>25 \mathrm{GeV} "$ : Missing Transverse Energy

2. if $\mathbb{E}_{T}<50 \mathrm{GeV}$, then the angle between any of the leptons or jets and the $\mathbb{Z}_{T}$ has to be at least 20 degrees.

3. $H_{t}>200 \mathrm{GeV}: H_{t}$ is the sum of all the transverse energies in the event. 
4. OS: The leptons must have opposite sign charges.

5. $Z$-veto: If an event is ee or $\mu \mu$ and has a di-lepton invariant mass between $76 \mathrm{GeV}$ and $106 \mathrm{GeV}$ it is rejected unless it has $\Delta \phi_{j}>10$ degrees and "jet significance", $\sigma_{j}>8.0$. $\Delta \phi_{j}$ is the minimum angle between the $\mathrm{E}_{T}$ and any of the tight jets in the events and $\sigma_{j}=\mathrm{E}_{T} / \sum_{k}\left(\mathrm{E}_{T} \cdot E_{t}^{k}\right)$. The sum is performed over all tight jets in the same hemisphere as the $\mathrm{E}_{T}$ vector.

\subsubsection{Trigger Requirements}

The triggering system looks for events with at least one high- $P_{T}$ lepton. Levels one and two require an EM calorimeter cluster, confirmed COT track, little to no energy deposited in the hadronic calorimeter for central and plug electrons. For a muon, the first and second level triggers require a hit in the muon chambers and a confirmed COT track. The level three

trigger requires that central electrons have an $E_{T} \geq 18 \mathrm{GeV}$. Pug electrons need to have $E_{T} \geq$ $20 \mathrm{GeV}$ and $\mathrm{E}_{T} \geq 15 \mathrm{GeV}$. Muons are required to have a stub and track- $P_{T} \geq 18 \mathrm{GeV} / \mathrm{c}$ by the level three trigger.

\subsection{Backgrounds for the Di-lepton Channel}

There are a number of processes that can imitate the di-lepton signature. They are predicted by the standard model and can appear as di-lepton events in the data stream, but were not produced by top-anti-top decay. The di-lepton channel has the lowest background of all the decay channels of $t \bar{t}$ events at CDF. The background sources that concern this analysis come from Diboson events (WW, WZ and ZZ), Drell Yan events that decay into pairs of leptons, $W \gamma$ events, and events that are referred to as Fake Di-lepton Events (or simply Fakes).

\subsubsection{Dibosons}

There are three types of diboson events: $W^{+} W^{-}, W^{ \pm} Z^{0}$, and $Z^{0} Z^{0}$. The first type, $W^{+} W^{-}$, is produced when the bosons decay leptonically and the jets come from higher order QCD processes: 


$$
p \bar{p} \rightarrow W^{+} W^{-}+2 j e t s+X \rightarrow l^{+} \nu l^{-} \bar{\nu}+2 j e t s+X .
$$

The number of events from this process, $N^{W^{+} W^{-}}$, can be calculated by

$$
N^{W^{+} W^{-}}=\epsilon^{W^{+} W^{-}} \cdot \sigma\left(p \bar{p} \rightarrow W^{+} W^{-}\right) \cdot B R\left(W^{+} W^{-} \rightarrow l^{+} \nu l^{-} \nu\right) \cdot \int \mathcal{L} d t
$$

where $\int \mathcal{L} d t$ is the integrated luminosity, $B R\left(W^{+} W^{-} \rightarrow l^{+} \nu l^{-} \nu\right)$ is the branching ratio for both of the $\mathrm{W}$ bosons to decay leptonically, $\sigma\left(p \bar{p} \rightarrow W^{+} W^{-}\right)$is the theoretical cross-section for $W^{+} W^{-}$production from $p \bar{p}$ collisions, and $\epsilon^{W^{+} W^{-}}$is the efficiency for the di-lepton selection to accept $W^{+} W^{-}$events.

The second type of diboson event, $W^{ \pm} Z^{0}$, can imitate a di-lepton event if the $Z^{0}$ decays into two high $P_{t}$ leptons, the $W^{ \pm}$decays hadronically, and the jet energies are mismeasured such that there is a large $\mathbb{E}_{T}$ :

$$
p \bar{p} \rightarrow W^{ \pm} Z^{0}+X \rightarrow q \bar{q}+l^{+} l^{-}+X \rightarrow l^{+} l^{-}+2 j e t s+X .
$$

The number of events from this process, $N_{1}^{W^{ \pm} Z^{0}}$, can be calculated in a similar manner to $N^{W^{+} W^{-}}$, given the appropriate values for the branching ratio, cross-section, and efficiency.

$$
N_{1}^{W^{ \pm} Z^{0}}=\epsilon^{W^{ \pm} Z^{0}} \cdot \sigma\left(p \bar{p} \rightarrow W^{ \pm} Z^{0}\right) \cdot B R\left(W^{ \pm} \rightarrow q \bar{q}\right) \cdot B R\left(Z^{0} \rightarrow l^{+} l^{-}\right) \cdot \int \mathcal{L} d t
$$

There is a second way in which $W^{ \pm} Z^{0}$ event can imitate di-lepton events:

$$
p \bar{p} \rightarrow W^{ \pm} Z^{0}+2 j e t s+X \rightarrow l^{ \pm} \nu+l^{+} l^{-}+2 j e t s+X
$$

In this case, the $\mathrm{W}$ boson also decays leptonically and 2 jets are formed from higher order QCD processes. Given the third lepton in this process, the likelihood that the event will pass the $Z_{0}$-veto cut is higher; however, the branching ratio of this process is three times lower than that of the first. Once again, the number of expected events, $N_{2}^{W^{ \pm} Z^{0}}$, can be calculated from:

$$
N_{2}^{W^{ \pm} Z^{0}}=\epsilon^{W^{ \pm} Z^{0}} \cdot \sigma\left(p \bar{p} \rightarrow W^{ \pm} Z^{0}\right) \cdot B R\left(W^{ \pm} \rightarrow l^{ \pm} \nu\right) \cdot B R\left(Z^{0} \rightarrow l^{+} l^{-}\right) \cdot \int \mathcal{L} d t
$$

The total contribution from $W^{ \pm} Z^{0}$ events is just the sum of each of the cases: $N^{W^{ \pm} Z^{0}}=$ $N_{1}^{W^{ \pm} Z^{0}}+N_{2}^{W^{ \pm} Z^{0}}$ 
The third type of diboson event, $Z^{0} Z^{0}$, can imitate a di-lepton event if one of the boson decays leptonically, while the other one decays hadronically, and the jet energies from the hardronic decays are mismeasured so that the $\mathrm{E}_{t}$ is large.

$$
p \bar{p} \rightarrow Z^{0} Z^{0}+X \rightarrow l^{+} l^{-}+q \bar{q}+X \rightarrow l^{+} l^{-}+2 j e t s+X
$$

The number of events expected from this process, $N^{Z^{0}} Z^{0}$, can be calculated from:

$$
N^{Z^{0} Z^{0}}=\epsilon^{Z^{0} Z^{0}} \cdot \sigma\left(p \bar{p} \rightarrow Z^{0} Z^{0}\right) \cdot B R\left(Z^{0} \rightarrow q \bar{q}\right) \cdot B R\left(Z^{0} \rightarrow l^{+} l^{-}\right) \cdot \int \mathcal{L} d t
$$

\subsubsection{Drell-Yan}

Events of this type can occur when $q \bar{q}$ annihilation produce a virtual $Z^{0}$ boson or a virtual photon. The $Z^{0}$ or photon then decays into either an $e^{+} e^{-}$or $\mu^{+} \mu^{-}$pair. When this is accompanied by two or more jets from higher order QCD processes, and the jet and lepton energies are mismeasured so that the $\mathrm{E}_{T}$ is large, then this type of event can imitate a di-lepton event.

$$
p \bar{p} \rightarrow Z^{0} / \gamma+2 j e t s+X \rightarrow l^{+} l^{-}+2 j e t s+X
$$

The $Z^{0}$-veto cut reduces this background significantly since most of the time the intermediate boson is a $Z^{0}$ and it tends to be on the $Z^{0}$ mass shell so that the di-lepton invariant mass, $M_{l^{+} l^{-}}$, will most likely be in the region $76<M_{l^{+} l^{-}}<106 \mathrm{GeV}$.

To calculate the number of Drell-Yan background events, $N^{D Y}$, the number of events inside the $Z^{0}$ mass window $\left(76<M_{l^{+} l^{-}}<106 \mathrm{GeV}\right)$ that pass the 2-jets and $\mathbb{E}_{T}$ di-lepton cuts, $N_{Z}^{\text {pass }}$, needs to be calculated. Additionally, the total number of $Z^{0}$ events inside the window before cuts, $N_{Z}$, the number of events below the window $\left(M_{l^{+} l^{-}}>76 \mathrm{GeV}\right)$ before cuts, $N_{l o}$, and the number of events above the window $\left(M_{l^{+} l^{-}}<106 \mathrm{GeV}\right)$ before cuts, $N_{h i}$, need to be calculated. Together, $N^{D Y}$ is given by:

$$
N^{D Y}=N_{Z}^{\text {pass }} \frac{\epsilon_{2 j}^{l o} N_{l o}+\epsilon_{2 j}^{h i} N_{h i}}{\epsilon_{2 j}^{Z} N_{Z}}
$$


where $\epsilon_{2 j}^{l o}, \epsilon_{2 j}^{Z}$, and $\epsilon_{2 j}^{h i}$ are all needed since the number of Drell-Yan events with two or more jets varies with $M_{l^{+} l^{-}}$. For each of the three regions defined by the $Z^{0}$ mass window, the fraction of Drell-Yan events with two or more jets can be approximated by a linear relation with $M_{l^{+} l^{-}}$. $\epsilon_{2 j}^{l o}$ is defined as the fraction of Drell-Yan events below the $Z^{0}$ mass window that corresponds to the average value of $M_{l^{+} l^{-}}$in that region. The quantities $\epsilon_{2 j}^{Z}$ and $\epsilon_{2 j}^{h i}$ are defined in a similar way.

$N^{D Y}$ as defined above will overestimate the number of background events because other types of events, specifically of interest $t \bar{t}$ events, will contribute to $N_{Z}^{\text {pass }}$. To correct for this, the number of $t \bar{t}$ events inside the $Z^{0}$ mass window that pass the cuts, $N_{\text {top }}^{Z}(l l)$, can be calculated by:

$$
N_{\text {top }}^{Z}(l l)=\left(1-\epsilon_{Z}\right) \cdot \int \mathcal{L} d t \cdot \sigma_{t \bar{t}} \cdot \epsilon_{D I L} \cdot R_{l l}
$$

where $l l$ is either ee or $\mu \mu$. $\epsilon_{Z}$ is the efficiency of the $Z^{0}$ mass cuts for $e^{+} e^{-}$and $\mu^{+} \mu^{-}, \sigma_{t \bar{t}}$ is the $t \bar{t}$ production cross-section, $\epsilon_{D I L}$ is the top acceptance in the di-lepton channel, and $R_{l l}$ is the $l^{+} l^{-}$acceptance relative to $\epsilon_{D I L}$ before the $Z^{0}$ mass cut. After calculating the $N_{\text {top }}^{Z}(l l)$, the total number of expected Drell-Yan background events, $N_{b g}^{D Y}$, can be determined by:

$$
N_{b g}^{D Y}=N^{D Y}-N_{t o p}^{Z}(e e)-N_{\text {top }}^{Z}(\mu \mu)
$$

There is a third type of Drell-Yan event where the initial boson decay goes into $\tau^{+} \tau^{-}$. Due to the short lifetime of the $\tau$, it decays into lighter leptons before it even passes through the first layer of the silicon detector. Such a process would look like this:

$$
q \bar{q} \rightarrow Z^{0}+X \rightarrow \tau^{+} \tau^{-}+X \rightarrow l^{+} \overline{\nu_{l}} \nu_{\tau} l^{-} \nu_{l} \overline{\nu_{\tau}}+X
$$

This process already has two light massive leptons and would only need two jets and enough $\mathrm{E}_{T}$ to resemble a di-lepton event. If a pair of jets from a higher order QCD process were involved in the event, the two $\tau$ leptons would not be back to back and the four neutrinos could contribute to a large $\mathrm{E}_{T}$. This would make a $Z^{0} \rightarrow \tau^{+} \tau-$ event look like a di-lepton event.

In order to calculate the number of expected $Z^{0} \rightarrow \tau^{+} \tau^{-}$events, $N_{\tau \tau}^{D Y}$, the acceptance needs to be calculated. While $Z^{0} \rightarrow \tau^{+} \tau^{-}$Monte Carlo samples are available, they are not expected to agree well with real data. So both MC and real data are used to calculate the acceptance: 


$$
\epsilon^{Z \rightarrow \tau \tau}=\epsilon_{E_{T}} \cdot \epsilon_{H_{T}} \cdot R_{M C} \cdot \frac{F_{2 j-\text { data }}}{F_{2 j-M C}}
$$

where $\epsilon_{E_{T}}$ is the acceptance with all cuts up to and including $\mathbb{E}_{T}, \epsilon_{H_{T}}$ is the acceptance of the $H_{T}$ cut, and $R_{M C}$ is the rejection factor from the 2-jet cut. $F_{2 j-M C}$ is the fraction of $Z^{0} \rightarrow \tau^{+} \tau^{-}$ events in the $\mathrm{MC}$ before any cuts, except lepton ID and isolation, that have two or more jets. Similarly, $F_{2 j-\text { data }}$ is the fraction of $Z^{0} \rightarrow e^{+} e^{-} / \mu^{+} \mu^{-}$events in the data before any cuts that have two or more jets. Once the acceptance is calculated, it can be used to estimate $N_{\tau \tau}^{D Y}$ :

$$
N_{\tau \tau}^{D Y}=\epsilon^{Z \rightarrow \tau \tau} \cdot \sigma\left(p \bar{p} \rightarrow Z^{0}\right) \cdot B R\left(Z^{0} \rightarrow \tau^{+} \tau^{-}\right) \cdot \int \mathcal{L} d t
$$

\subsection{3 $W \gamma$}

The $W \gamma$ channel is the smallest of the backgrounds that concern this analysis. In this channel, the $W^{ \pm}$decay leptonically into either an $e^{ \pm}$or $\mu^{ \pm}$. Jets can either come from higher order QCD processes or from the $\gamma$ conversion. The second lepton comes from a jet that results in either an $e^{ \pm}$or $\mu^{ \pm}$. The $\mathbb{E}_{T}$ would come from the mismeasurement of any jets in the event. The following describes such a process:

$$
p \bar{p} \rightarrow W^{ \pm}+\gamma+j e t s+l^{ \pm}+X \rightarrow l^{ \pm} \nu+j e t s+j e t s+l^{ \pm}+X
$$

and the number of expected events would be calculated by:

$$
N^{W \gamma}=\epsilon^{W \gamma} \cdot \sigma\left(p \bar{p} \rightarrow W^{ \pm} \gamma\right) \cdot B R\left(W^{ \pm} \rightarrow l^{ \pm} \nu\right) \cdot \int \mathcal{L} d t
$$

\subsubsection{Fakes}

The final type of background that concerns this analysis happens to also be the largest. "Fake Events" are events where a jet is misidentified as a lepton, and therefore the jet is a "Fake Lepton." If a jet has a single high $P_{t}$ track and deposits a significant amount of energy in the electromagnetic calorimeter, it could be identified as an electron. If a jet produces a pion or kaon that decays leptonically with a muon in the final state, it could fake a muon that came 
from a process like $W^{ \pm}$decay. $t \bar{t}$ events that decay in the lepton plus jets channel can result in this type of fake event when one of the jets from the hadronic decay of the $W$ boson looks like a good lepton.

To estimate the number of fake events, $N^{\text {fake }}$, the real data is used. W+jets events are given a fake rate probability by weighting the jet candidates in the event. The fake rate is the probability that an object that has both the characteristics of a jet and of a high $P_{t}$ lepton could be reconstructed as a good lepton. A fakeable object is defined as either a CDF_EM Object with $E_{\text {had }} / E_{E M}<0.125$ or a CDF Muon with $E / p<1$. An event must also have at least one "trigger" jet with raw $E_{t}>50 \mathrm{GeV}$, and the fakeable object be reconstructed outside a cone of $\Delta R<0.4$ around the "trigger" jet.

An event is considered "W+fakeable" if it has one and only one good central high $P_{t}$ lepton, $\mathrm{E}_{T}>25 \mathrm{GeV}$, and a jet that is classified as a second fakeable object. If this is the case, the jet that is the second fakeable object is treated as the second lepton in a di-lepton event, and is subject to the di-lepton selection. Tight jets found in a cone of $\Delta R<0.4$ are dropped from the tight jets counting for that event.

\subsubsection{Background Summary}

Table 13 and Table 14 give the summary of the total expected number of events in $2.0 \mathrm{fb}^{-1}$ of data collected at Fermi Lab from the CDF experiment for each category of background and signal after all di-lepton selection cuts were applied. Additionally it shows the MC data sets that were used to determine the acceptance of each category where applicable. 


\begin{tabular}{lcc}
\hline Source & Data Set & Number of Events \\
\hline \hline Fakes & W+jets data & $21.75 \pm 6.33$ \\
$\mathrm{DY} \rightarrow e e+\mu \mu$ & zewkad + zewk9m & $12.78 \pm 2.17$ \\
$\mathrm{WW}$ & itopww & $6.81 \pm 1.17$ \\
$\mathrm{DY} \rightarrow \tau \tau$ & zewkat & $5.26 \pm 1.02$ \\
$\mathrm{WZ}$ & itopwz & $1.59 \pm 0.26$ \\
$\mathrm{ZZ}$ & itopzz & $1.09 \pm 0.85$ \\
$\mathrm{~W} \gamma$ & rewk28 + rewk29 & $0.17 \pm 0.18$ \\
\hline Total Background & & $49.45 \pm 7.83$ \\
\hline$t \bar{t}(\sigma=6.7$ pb) & ttop75 & $93.86 \pm 7.14$ \\
Total SM Expectation & & $143.31 \pm 13.09$ \\
\hline \hline Events Found in Data & & 145 \\
\hline
\end{tabular}

Table 13: Signal and background event expectations predicted by the Standard Model.[3]

\begin{tabular}{l|c|c|c||c}
\hline Source & ee & $\mu \mu$ & $\mathrm{e} \mu$ & $\mathrm{ll}$ \\
\hline \hline Fakes & $2.79 \pm 0.95$ & $6.54 \pm 2.22$ & $12.42 \pm 3.91$ & $21.75 \pm 6.33$ \\
$\mathrm{DY} \rightarrow e e+\mu \mu$ & $6.63 \pm 1.47$ & $5.17 \pm 1.38$ & $0.98 \pm 0.44$ & $12.78 \pm 2.17$ \\
$\mathrm{WW}$ & $1.56 \pm 0.29$ & $1.76 \pm 0.33$ & $3.48 \pm 0.61$ & $6.81 \pm 1.17$ \\
$\mathrm{DY} \rightarrow \tau \tau$ & $1.19 \pm 0.24$ & $1.28 \pm 0.26$ & $2.79 \pm 0.55$ & $5.26 \pm 1.02$ \\
$\mathrm{WZ}$ & $0.67 \pm 0.11$ & $0.49 \pm 0.09$ & $0.43 \pm 0.08$ & $1.59 \pm 0.26$ \\
$\mathrm{ZZ}$ & $0.46 \pm 0.36$ & $0.46 \pm 0.36$ & $0.16 \pm 0.13$ & $1.09 \pm 0.85$ \\
$\mathrm{~W} \gamma$ & $0.17 \pm 0.18$ & $0.00 \pm 0.00$ & $0.00 \pm 0.00$ & $0.17 \pm 0.18$ \\
\hline Total Background & $13.48 \pm 2.19$ & $15.70 \pm 2.89$ & $20.27 \pm 4.23$ & $49.45 \pm 7.83$ \\
\hline$t \bar{t}(\sigma=6.7$ pb) & $20.54 \pm 1.58$ & $22.70 \pm 1.74$ & $50.62 \pm 3.86$ & $93.86 \pm 7.14$ \\
Total SM Expectation & $34.02 \pm 3.39$ & $38.41 \pm 3.96$ & $70.89 \pm 6.69$ & $143.31 \pm 13.09$ \\
\hline \hline Events Found in Data & 30 & 42 & 73 & 145 \\
\hline
\end{tabular}

Table 14: Signal and background event expectations predicted by the Standard Model by lepton flavor.[3] 


\section{Chapter 5 - The Dalitz and Goldstein Method}

\section{$5.1 \quad$ Introduction}

This Chapter will give an exposition of the Dalitz and Goldstein method[11] of measuring the mass of the top quark in the di-lepton channel. It is based on a geometrical construction and the leading order matrix elements for $t \bar{t}$ production, $t \rightarrow W b$ decay, and $W \rightarrow l \nu$ decay, which defines a likelihood. The method has been modified to also work in the lepton plus jets channel by Dalitz, Goldstein, and Sliwa[12][13][14][15]. A major advantage of this method is the inclusion of both $q \bar{q} \rightarrow t \bar{t}$ and $g g \rightarrow t \bar{t}$ matrix elements and its lack of dependence on $\mathrm{E}_{T}$, a point that is stressed by Sliwa in reference[16].

The original method was improved significantly in 1999 by Karr and Sliwa[7]. This thesis employs a much-improved technique, a result of careful studies performed over the past few years.

\subsection{Geometrical Construction}

The Dalitz and Goldstein[11] method for measuring the top quark mass in the di-lepton channel employs a geometrical interpretation of the equations of constraint.

Those equations are:

$$
\begin{aligned}
\left(l^{+}+\nu\right)^{2} & =M_{W}^{2} \\
\left(l^{-}+\bar{\nu}\right)^{2} & =M_{W}^{2} \\
\left(t-l^{+}-b\right)^{2} & =M_{\nu}^{2}=0 \\
\left(\bar{t}-l^{-}-\bar{b}\right)^{2} & =M_{\nu}^{2}=0 \\
t^{2}=M_{t}^{2} & =M_{\bar{t}}^{2}=\bar{t}^{2} \\
-P_{x}^{t} & \sim P_{x}^{\bar{t}} \\
-P_{y}^{t} & \sim P_{y}^{\bar{t}}
\end{aligned}
$$

where $t, l, b$, and $\nu$ are the top quark, lepton, bottom quark, and neutrino 4-momenta. $M_{W}$, $M_{t}$, and $M_{\nu}$ are the masses of the $\mathrm{W}$ boson, top quark, and neutrino. $P_{x}^{t}$ and $P_{y}^{t}$ are the $\mathrm{x}$ and y components of the top and anti-top quarks' transverse momenta. Equations 43 and 44 are 
only approximate and are considered "weak" constraints while the rest are "hard" constraints. Equations 43 and 44 are "weak" due to the possibility that the partons inside of the protons may have some transverse momentum. If this were not the case, then both equations 43 and 44 would be "hard" constraints.

The geometrical construction begins by rewriting equations 38 through 41 in terms of the top and bottom quark kinematics

$$
\begin{aligned}
\left(\vec{P}_{t}-\vec{P}_{b}\right)^{2} & =\left(E_{t}-E_{b}\right)^{2}-M_{W}^{2} \equiv R_{W}^{2}, \\
\left(\vec{P}_{t}-\vec{P}_{b}-\vec{P}_{l^{+}}\right)^{2} & =\left(E_{t}-E_{b}-E_{l^{+}}\right)^{2} \equiv R_{\nu}^{2} .
\end{aligned}
$$

$\vec{P}_{t}, \vec{P}_{b}$, and $\vec{P}_{l^{+}}$are the 3-momenta for the Top, Bottom, and charged lepton; and $E_{t}, E_{b}$, and $E_{l^{+}}$are their corresponding energies. A second pair of equations can be written for the anti-top quark and its decay products in exactly the same way. These equations have a similar form to the equation for a sphere, in this case in 3-dimensional momentum space.

$$
X^{2}+Y^{2}=R^{2}
$$

The centers of these two spheres are separated by the charged lepton's 3-momentum $\vec{P}_{l^{+}}$. Figure 9 shows an example of two non-intersecting spheres that have their centers separated by $\vec{P}_{l^{+}}$. Both $\vec{P}_{l^{+}}$and $\vec{P}_{b}$ in this picture are fixed because they are measured quantities; however, $\vec{P}_{t}$ is not measured and free to move about. Since the two spheres in Figure 9 do not intersect, it is not possible to draw a $\vec{P}_{t}$ vector that satisfies Eq. 45 and 46 simultaneously. Figure 10 shows two spheres that intersect at a single point. In this case, there exists one $\vec{P}_{t}$ vector that will satisfy both equations. This is a special case that will be discussed more in the next paragraph. Figure 11 shows a more generic case where the two spheres intersect at more than one point. In this case, the intersection is a circle. As long as $\vec{P}_{t}$ lies on this circle, it will satisfy both equations.

If the lepton masses are neglected, the top quark energy will be constant on this circle with radius $r$ (Figure 12).

$$
r^{2}=\frac{M_{W}^{2}}{\left|\overrightarrow{P_{l^{+}}}\right|}\left(E_{t}-E_{o}\right)
$$




$$
\begin{aligned}
& R_{W}^{2} \equiv\left(\vec{P}_{t}-\vec{P}_{b}\right)^{2} \\
& R_{v}^{2} \equiv\left(\vec{P}_{t}-\vec{P}_{b}-\vec{P}_{l^{+}}\right)^{2}
\end{aligned}
$$

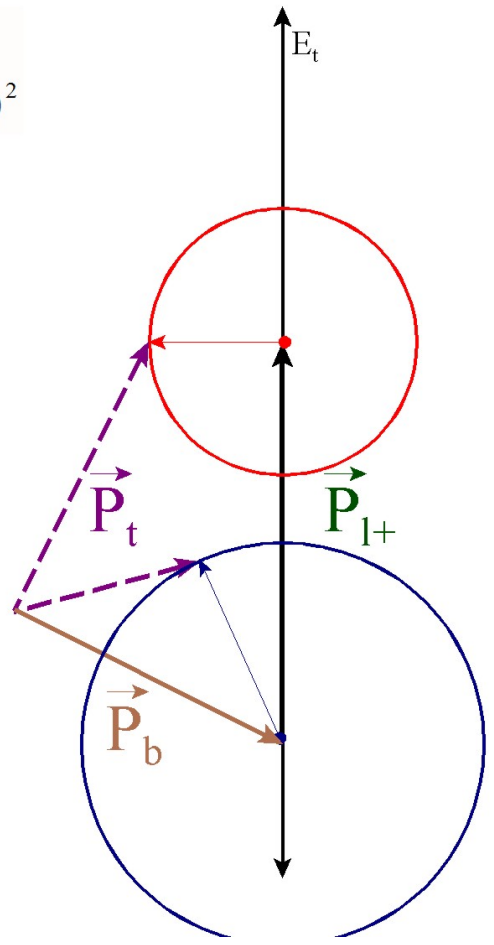

Figure 9: The two spheres of radii $R_{W}$ and $R_{\nu}$, whose centers are separated by $\vec{P}_{t}$, but do not intersect.

$$
E_{o}=E_{b}-E_{l}+\frac{M_{W}^{2}}{4 E_{l}}
$$

$E_{0}$ is the lowest possible energy for the top quark given the kinematics of the event. If $E_{t}$ is equal to $E_{0}$, this corresponds to the special case mentioned above. If $E_{t}$ is less than to $E_{0}$, the two spheres will not intersect and therefore give no solution.

Given a different top quark energy $E_{t}$, a different pair of spheres can be constructed. For each pair of spheres that intersect, a new circle is created. The top quark energy will increase in the direction of $\vec{P}_{l^{+}}$. These circles form the surface of a paraboloid (Figure 13). While the top quark energy is constant on these circles, the top quark mass, $M_{t}$, is not. However, if the mass is fixed the top quark 3-momentum vector will be confined to a conic section of the paraboloid which happens to be, out of the four possible conic section types, an ellipse. The orientation and eccentricity of the ellipse will depend on the assumed mass and the 4-momenta of the leptons and Bottom Quarks (Figure 14). A similar ellipse can be constructed in the same way for the anti-top quark. 


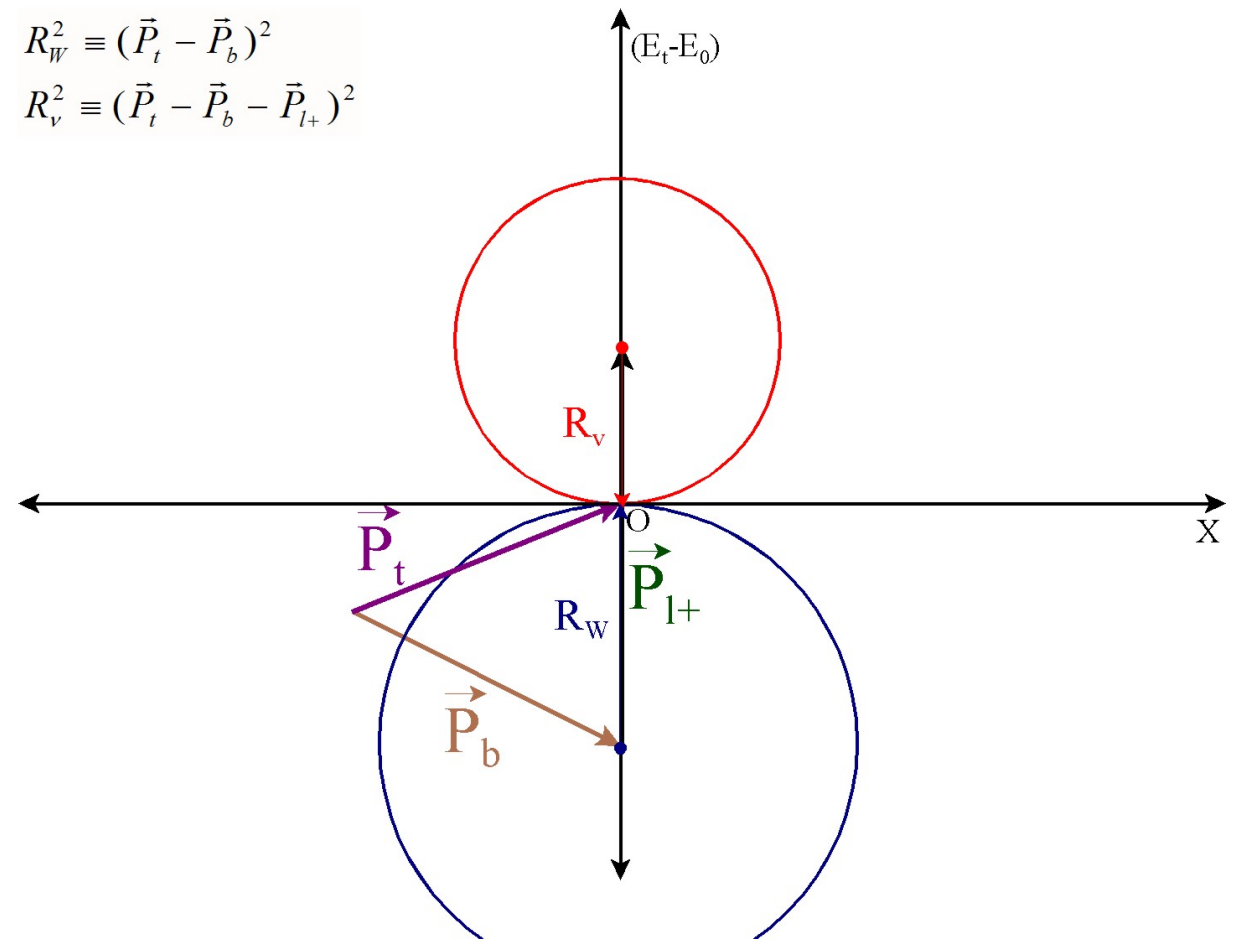

Figure 10: The two spheres of radii $R_{W}$ and $R_{\nu}$, which intersect at a point. $\mathrm{O}$ is the point where $E_{t}-E_{0}=0$, the minimum energy allowed for the top quark as determined by the kinematics of the event.

Once both ellipses are constructed, they are projected into the transverse plane of the detector, one of which is reflected about the origin. Using the "weak" constraints, Eq. 43 and Eq. 44, each pair of points, one from each ellipse, will correspond to a possible solution consistent with the assumed top mass and the measured lepton and jet momenta. If these final constraints were "hard", then only the points of intersection of the two ellipses would need to be considered as possible solutions. It is because they are "weak" that every pair of points needs to be considered. Given a pair of points from the projection of the two ellipses, the transverse momentum, $P t_{t \bar{t}}$, of the top-anti-top system can be calculated. Each pair of points is assigned a likelihood factor, $P\left(P t_{t \bar{t}}\right)$, from the $P t_{t \bar{t}}$ spectrum. The expected shape of the $P t_{t \bar{t}}$ distribution is calculated from Monte Carlo simulation. If a different top mass is assumed, a different pair of ellipses will be created whose projections into the $\mathrm{x}-\mathrm{y}$ plane will give another set of possible solutions. 


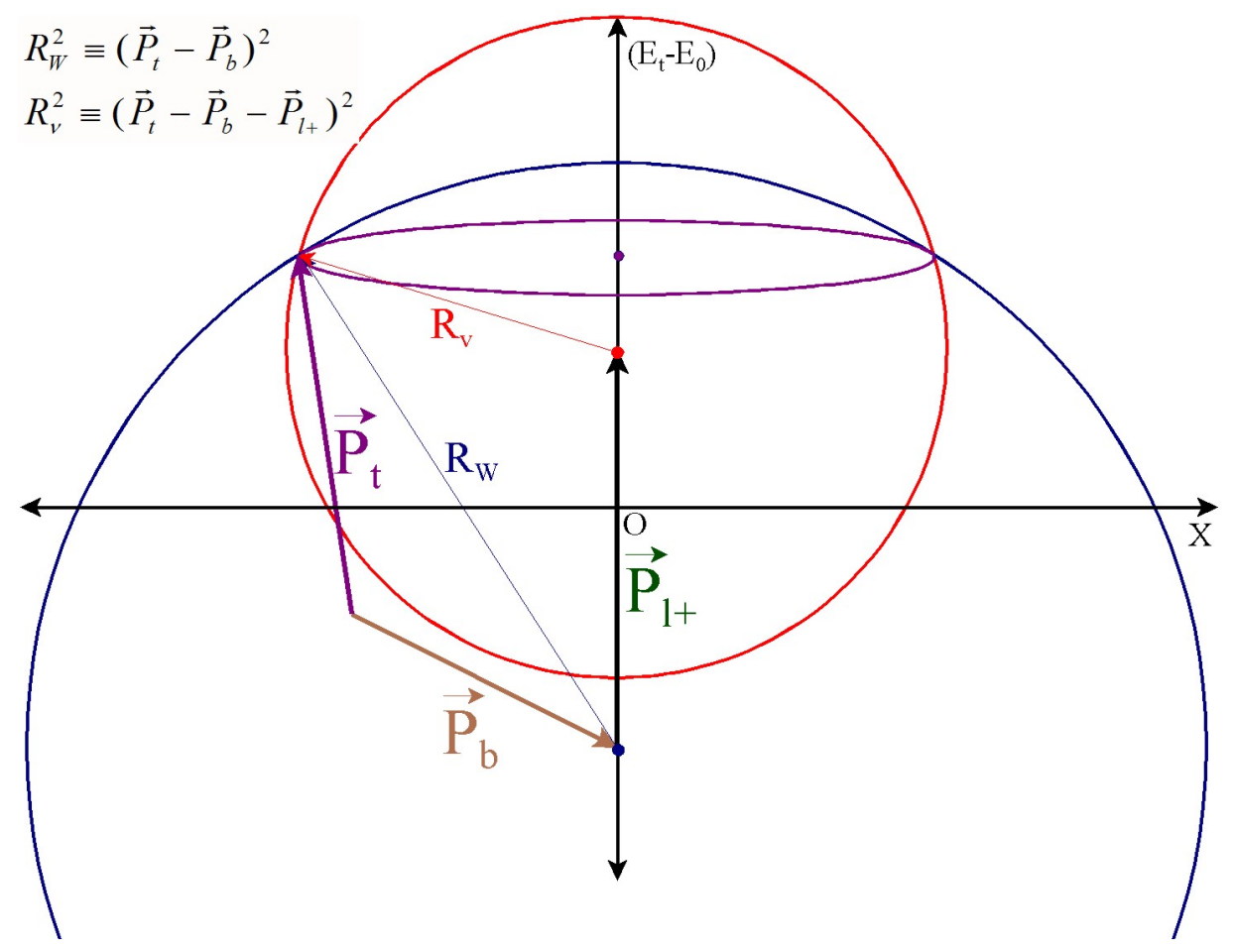

Figure 11: The two spheres of radii $R_{W}$ and $R_{\nu}$, showing a circle of intersection.

\subsection{The Likelihood}

To determine the most likely mass of the top quark, a probability distribution is constructed for each combination of leptons and jets in an event. Given an assumed top quark mass, a likelihood value, $L_{i}$, for all pairs of points on the families of ellipses are projected onto the $M_{t}$-axis of this distribution. The most likely top quark mass corresponds to the peak of this distribution. The likelihood values, $L_{i}$, are a product of six probability factors.

$$
L_{i}=P\left(P_{t \bar{t}}\right) \times G(b) \times G(\bar{b}) \times P\left(x_{1}, x_{2}\right) \times P\left(l^{+}\right) \times P\left(l^{-}\right)
$$

$P\left(P t_{t \bar{t}}\right)$ is the factor related to the transverse momentum of the top-anti-top system $G(b)$ and $G(\bar{b})$ are the factors related to Jet Energy Smearing

$P\left(x_{1}, x_{2}\right)$ is the factor related to the leading order matrix element for the $t \bar{t}$ production. $P\left(l^{+}\right)$and $P\left(l^{-}\right)$are the factors resulting from $\mathrm{V}-\mathrm{A}$ Calculations with the matrix elements for the $t \rightarrow W b$ and $W \rightarrow l \nu$ decays 


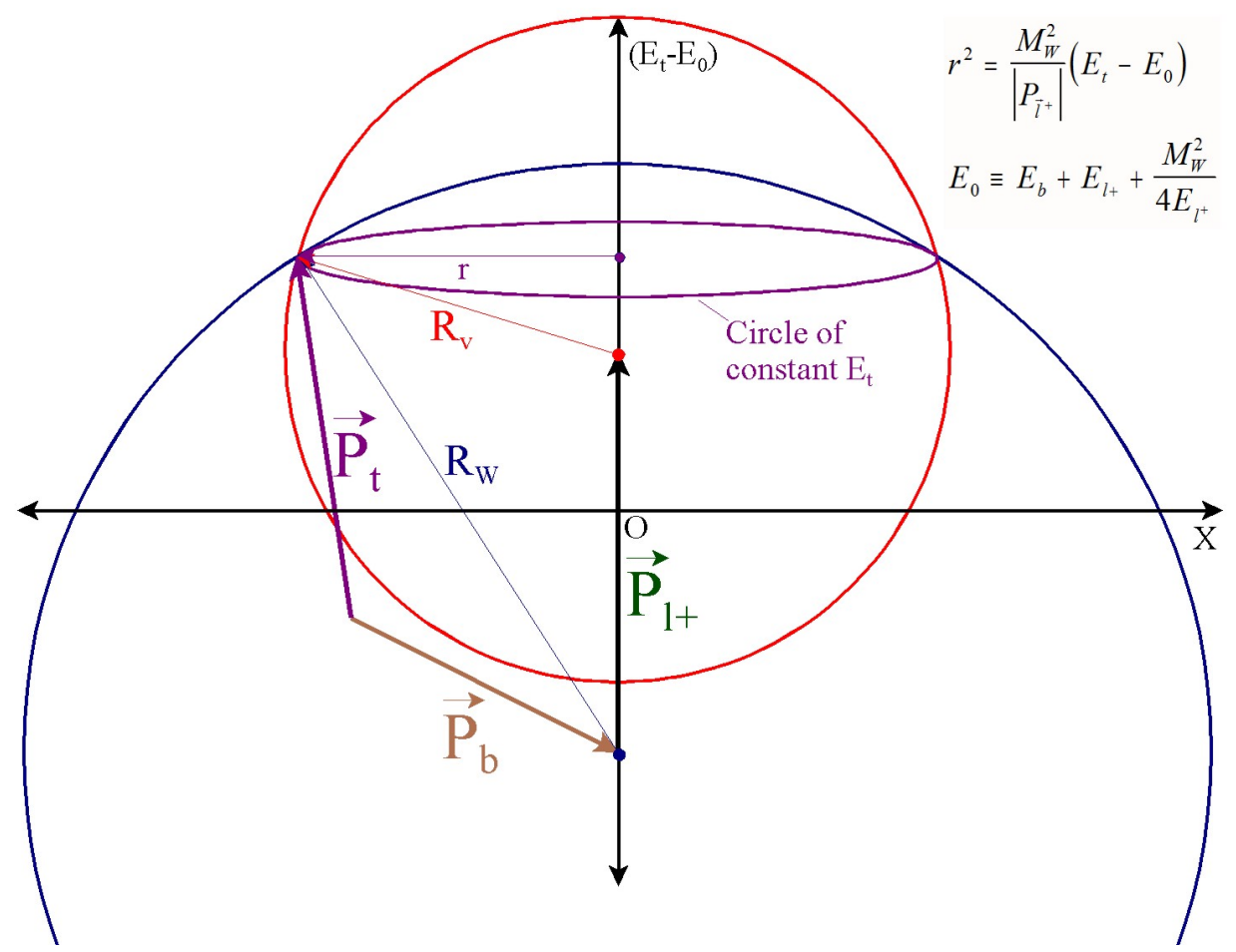

Figure 12: The two spheres of radii $R_{W}$ and $R_{\nu}$ showing a circle of intersection with constant energy $E_{t}$.

i. $P\left(P t_{t \bar{t}}\right)$

Given a pair of points from the projection of the two ellipses, the transverse momentum, $P t_{t \bar{t}}$, of the top-anti-top system can be calculated. Each pair of points is weighted by a likelihood factor, $P\left(P t_{t \bar{t}}\right)$, from the $P t_{t \bar{t}}$ spectrum. The expected shape of the $P t_{t \bar{t}}$ distribution is calculated from Monte Carlo simulation. If a different top mass is assumed, a different pair of ellipses will be created whose projections into the $\mathrm{x}-\mathrm{y}$ plane will give another set of possible solutions.

i. $P\left(x_{1}, x_{2}\right)$

A relative likelihood factor is assigned that describes the level of agreement between the Feynman-x values, $x_{1}$ and $x_{2}$, that are calculated from the event and those predicted by theory, i.e. the dependence of the leading order matrix element on the structure functions for the event, 


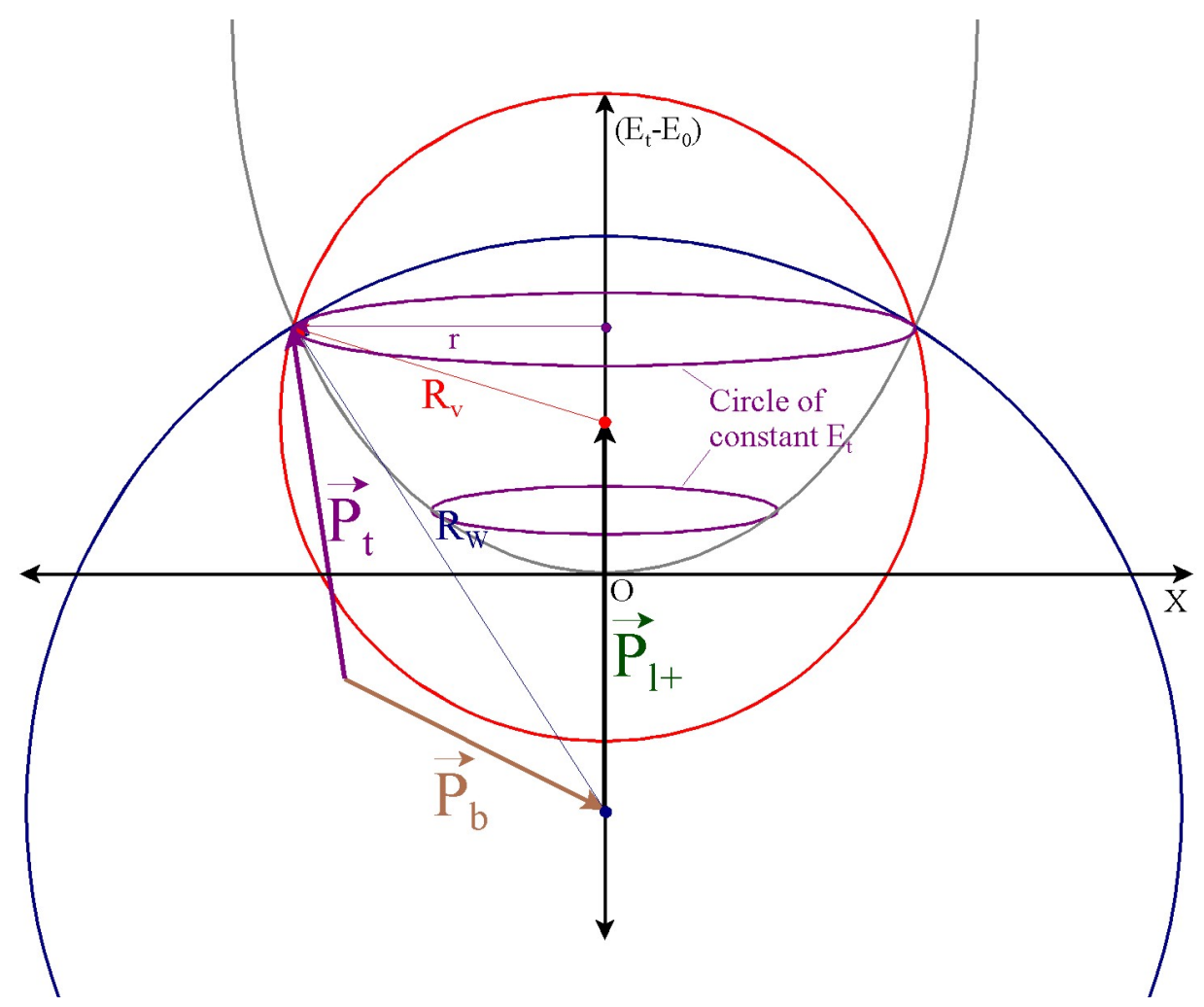

Figure 13: The two spheres of radii $R_{W}$ and $R_{\nu}$ showing a circle of intersection with constant energy $E_{t}$. Also seen here is a second circle of constant $E_{t}$ resulting from the intersection of two other spheres (not drawn). Both circles begin to form the paraboloid surface.

$$
\begin{aligned}
P_{x_{1}, x_{2}} & =\frac{\sum_{i=q q, g g} F_{i}\left(x_{1}\right) F_{i}\left(x_{2}\right) \frac{d \sigma}{d \hat{t}}(\hat{s}, \hat{t})_{i}}{\sum_{i=q q, g g} \frac{d \sigma}{d \hat{t}}(\hat{s}, \hat{t})_{i}} \\
x_{1,2} & =\left(E_{t}+E_{\bar{t}} \pm\left(t_{L}+\bar{t}_{L}\right)\right) / 2 P \\
\hat{s} & =x_{1} x_{2} s \\
\hat{t} & =M_{t}^{2}-x_{1} \sqrt{s}\left(E_{t}-t_{L}\right)
\end{aligned}
$$

where $i$ labels the $q \bar{q}$ and $g g$ processes; $F_{i}$ are the structure functions; $\hat{s}$ is the parton-parton center-of-mass energy; $\hat{t}$ is the momentum transfer of the top-anti-top quark production subprocess; $P$ is the proton momentum; $s$ is the square of the energy in the proton-anti-proton system; and $t_{L}$ is the longitudinal momentum of the top quark in the lab frame of the proton-anti-proton system.

iii. $P\left(l^{+}\right)$and $P\left(l^{-}\right)$ 


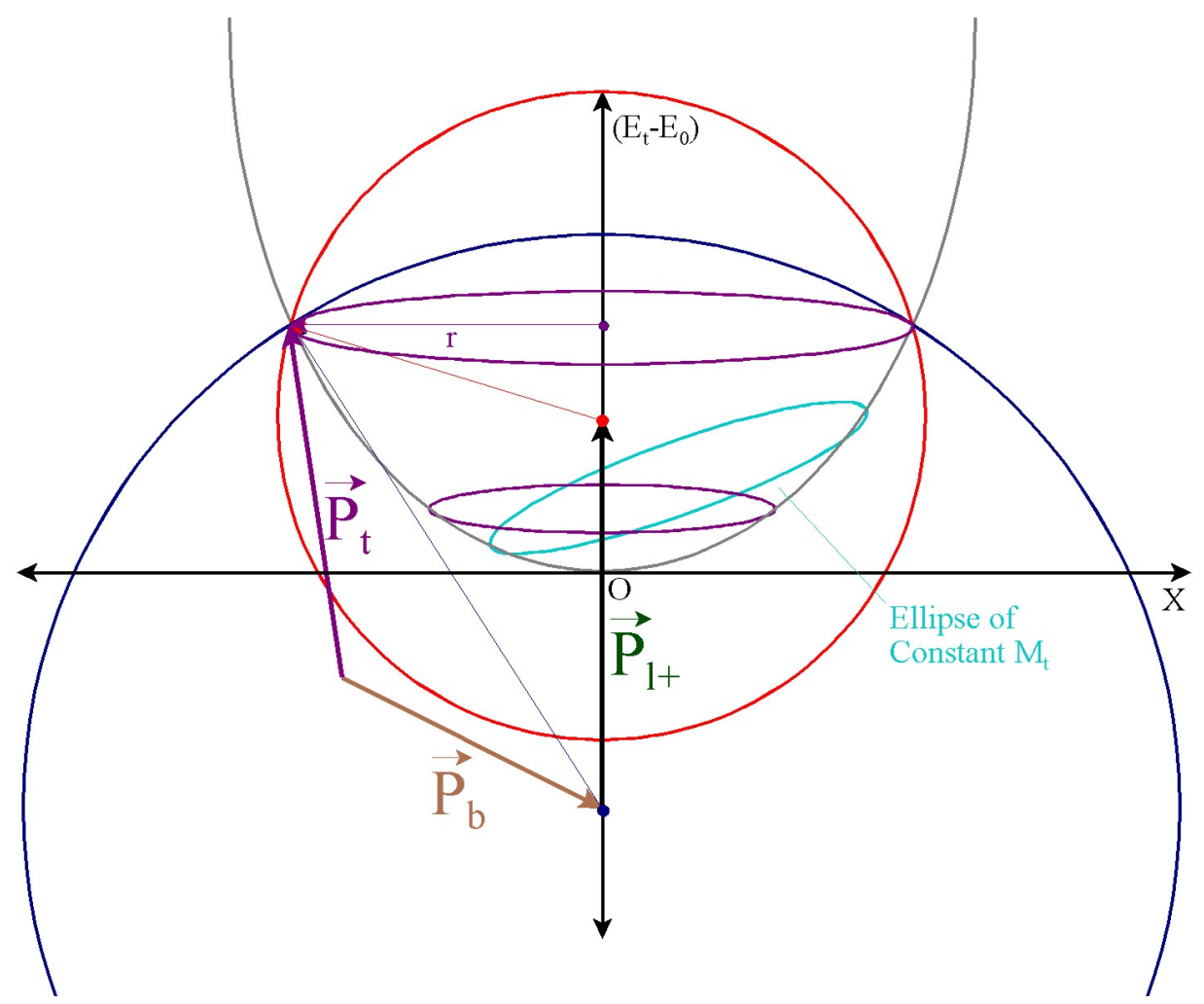

Figure 14: The two spheres of radii $R_{W}$ and $R_{\nu}$ showing a circle of intersection with constant energy $E_{t}$. Assuming a constant $M_{t}$ confines $P_{t}$ to a conic section of the paraboloid which is an ellipse.

For each point a likelihood factor is calculated to describe the agreement of the charged lepton energies calculated in the top quark rest frame with the values predicted by $\mathrm{V}$-A calculations of the $t \rightarrow W+b$ and $W \rightarrow l+\nu$ decays.

$$
d P\left(E_{l}\right)=\left(24 / M_{t}^{2}\right) E_{l}\left(1-2 E_{l} / M_{t}\right) d E_{l}
$$

iv. $G(b)$ and $G(\bar{b})$

Since the $b$-jet and $b$-quark measured energies have large errors, the true parton energies can differ from the measured values. A range of energies is defined which is centered about the measured energy of each of the jets in an event. The range is chosen to be $3 \sigma$, where $\sigma$ is the width of the jet energy resolution distribution. A probability, $G(b)$, is assigned for each point within the $3 \sigma$ range. Points that correspond to $b$-jet energies that deviate from the measured value will be downgraded by a Gaussian probability factor giving them a lower probability 
than ones closer to the measured value. This smearing of the jet energies will give a family of ellipses for each jet. Each combination from an event with different jets will have its own pair of sigmas, one for each jet in the combination. The number of discrete points is set to the same value for all jets even though their sigmas will vary. In order to compensate for this, the likelihoods are first multiplied by the product of these sigmas when comparing the likelihood of one combination to another.

Pairing together all the combinations of ellipses from each family will create a 2-dimensional grid where the axes are indexed by the smeared jet energies. Each point on the grid will have a value which is the $L_{i}$ that corresponds to smeared jets momenta, the given lepton momenta, and assumed top mass. There will be a different grid for each assumed top mass. Altogether, this will create a 3-dimensional space where the third axis is the assumed top mass. Each point in this space will have a value that comes from the sum of all the pairs of points on the pair of ellipses that correspond to the given assumed top mass and the two smeared jet energy values. The ellipses will vary in size. Geometrically speaking, each ellipse has an infinite number of points, but in the world of computers an infinite number of points can not be summed. Thus, a discrete set of points needs to be defined for each ellipse. This can be done in two ways. The first is to choose the number of points for all ellipses and allow the distance between points to be variable. The second way is to hold the distance between each point on an ellipse constant, so the number of points in the sum will be its circumference divided by the pre-defined distance between points. The first way has a significant problem. The most important points in the sum over the pairs of points on the ellipses are the intersection points of the two ellipses. If the distance between points is allowed to be variable, and an ellipse is large, it becomes more likely that the intersection point and the points around the intersections will be missed. The advantage here is that the number of points that are being considered can be kept small and thus reduces the amount of CPU time needed to do the analysis. This was the method used by Karr and Sliwa[7] in their measurement in 1999. The second method has two main drawbacks. If an ellipse is small, it will have very few points, possibly as little as one point, and if an ellipse if very large, it will take a significant amount of CPU time to compute the sum. However, there should only be small ellipses around the minimum allowed assumed top mass, and modern computers can compute these sums in a reasonable amount of time. The second method is superior in that it will guarantee integration near the intersection points given a small enough 
choice of the spacing. As newer and faster computers are created, a smaller spacing can be used which should help to improve the integration over the ellipses and thus improve this method of measuring the top quark mass. This is one of the important changes made in this measurement from the earlier measurement. Summing over the assumed top mass axis of this space will reduce it back to a 2-dimensional grid with the smeared jet energies as the remaining axes; however, now the value at each point is a total likelihood. The point on the grid with the greatest total likelihood will be chosen as the most probable solution given this combination of leptons and jets.

It should be noted that this is another important difference in methodology between this analysis and the Run 1 analysis done by Karr and Sliwa[7]. In that analysis, the 2-D smeared jet energy grid was integrated over to get the total likelihood for the event instead of picking the best combination out of all possible smeared jet combinations.

This total likelihood and its corresponding probability distribution will be compared to other combinations of leptons and jets (Bottom Quarks) from the same event where the greater one is favored.

\subsection{MET Probability}

There is an additional probability factor calculated for each combination based on the measured missing transverse energy. During the analysis of each combination, the energy and momentum of the neutrinos are obtained. The difference between the two missing neutrino $\mathrm{x}$ and y momenta in the transverse plane and the missing transverse energy $\mathrm{x}$ and $\mathrm{y}$ components are compared and assigned a probability based on the shape of the reconstructed $\mathbb{E}_{T} \mathrm{x}$ and y components from MC events.

\subsection{The Joint Likelihood}

Once a combination from an event is chosen via the method described above, and all events have been analyzed, a joint likelihood is created. The joint likelihood is the product of the probability distributions from the chosen combinations from each event. Since the true top mass for all of the events is the same, the joint probability distribution reveals where all the 
events are most consistent in $M_{t}$, which should point toward the true mass. The shape of the probability distribution for each event will not necessarily be the same. Some will be asymmetrical and some may have more than one peak. Choosing the mean or the peak value as the top mass for an individual event may include biases from the shape of the distribution. By taking the product of the event distributions, the joint distribution becomes more Gaussian in shape. Multiple peaks and asymmetries in the individual events are eliminated in the joint distribution as are the possible biases that these characteristics may produce. The arithmetic mean of the joint distribution is the top quark mass, the most likely value of the top quark Mass consistent with all of the events in the sample.

\subsection{The Construction of the Pseudo-Experiments}

The real data and the Monte Carlo data are treated in exactly the same way in every detail of this analysis except for the method of constructing the joint likelihood. When analyzing real data, the likelihood distributions from all of the candidate events take part in the product that forms the Joint Likelihood. When analyzing the Monte Carlo data, all of the events that pass the cuts and have a solution are put into a pool of events. Next, events are randomly selected from the pool and their likelihood distributions are multiplied together to produce the joint likelihood, which forms one pseudo-experiment. The number of events selected from the pool to form one pseudo-experiment depends on the number of candidate events expected in the real data. The number of events is randomly generated based on a Poisson distribution whose mean is the number of candidate events. Care is taken to not allow an event to appear more than once in the same pseudo-experiment; however, all events in the pool are available to every pseudo-experiment. The mean and RMS from each pseudo-experiment are used to calculate the delta and the pull. The mean, delta, pull, and RMS are each put into histograms to produce their own distributions.

\subsubsection{The RMS Correction}

When building the pseudo-experiments using the joint likelihood method described above, it becomes necessary to make a correction to the RMS in order to relate it to the errors. If a simple distribution populated by the means of the events in a pseudo-experiment was used to make 
the measurement of the mass, the error on that measurement would be related to the RMS of the distribution by $1 / \sqrt{N}$. This is, however, not the case for a joint likelihood distribution. A joint likelihood distribution is too narrow and a correction factor is needed.

In order to explain how the correction factor is calculated, a simple model is needed. The model is a box filled with cards, and on each card is a number. The number of cards in the box is $N$. The sum of all the numbers on the cards is $S_{b o x}$, the average of all the numbers is $A_{b o x}$ and the standard deviation of the numbers in the box is $\sigma_{b o x}$. Now, $n$ cards are drawn from the box at random without replacement. The expectation value for the sum of the draws, $S_{d r a w}^{E V}$, is $n * A_{b o x}$ and the standard error on the sum of the draws from the box is $\sqrt{n} * \sigma_{b o x}$. The expectation value for the average of the draws, $A_{d r a w}^{E V}$, is $S_{d r a w}^{E V} / n$ which is simply $A_{b o x}$ and the standard error for the average of the draws is $\sqrt{n} * \sigma_{b o x} / n$ which simplifies to $\sigma_{b o x} / \sqrt{n}$.

The pool of events in this analysis is very similar to the box of cards. Each event is a card and the number on the card is the mean of the distribution that corresponds to the event. All of the same statistics that applied to the box of cards will apply to the pool of events in exactly the same way. However, in this analysis, the average is not taken, but instead a joint distribution is made. While at first glance this seems to be very different, making a joint distribution out of the individual mass distributions for each event is like taking the average of the numbers on the cards. So, the standard error for a joint distribution should be similar to that of the random draws from a box and the RMS of a joint distribution is related to that error.

$$
R M S_{J D} \propto \sigma_{\text {pool }} / \sqrt{n}
$$

If all possible combinations of $n$ events were drawn from the pool and the mean of each sample was put into a histogram, that histogram would be a total probability distribution. It would be normal in shape even if the parent distribution is not. Its mean would be similar to the mean of the parent distribution and its width would be the error on the mean of the sample distributions. Since the number of possible combinations is an extremely large number, it is not practical to compute every possible combination. Instead, it is sufficient to draw $n$ events at random from the pool $X$ number of times as long as $X$ is large. When this is done, the resulting histogram will not be a complete total probability histogram, however its mean and width, $\sigma_{X P E}$, will be the same as if all possible combinations were sampled. Since the widths 
of these distributions are the same, $\sigma_{X P E}$ describes the error on the PEs.

$$
\sigma_{X P E} \approx \sigma_{p o o l} / \sqrt{n}
$$

It follows that

$$
R M S_{J D} \propto \sigma_{X P E}
$$

This gives the correction factor, $C_{r m s}$, to the RMS of the joint distributions.

$$
C_{r m s}=\sigma_{X P E} / R M S_{J D}
$$

The corrected width will be

$$
R M S_{\text {corr }}=C_{\text {rms }} \times R M S_{\text {raw }}
$$

where $R M S_{\text {corr }}$ and $R M S_{\text {raw }}$ are the corrected and uncorrected widths of the individual joint distributions of each PE.

Figures 15 and 16 show that $C_{R M S}$ is independent of the top mass.

The value of $C_{r m s}$ has a dependence, discussed in more detail in Section 5.7, on the shapes of the likelihood distributions from the events.

\subsubsection{The Mapping Function}

Analyses of a range of top mass MC samples are used to study the correlation between the generated and reconstructed mass. Each mass MC sample has its own mean, delta, pull, and RMS distribution. Given the mean distribution for each mass MC sample, a plot of reconstructed mass as a function of MC mass is made. While it is possible to fit various shapes to the points, a line does the job very well. That fit is the mapping function that will be used to make the final correction to the measurement. 


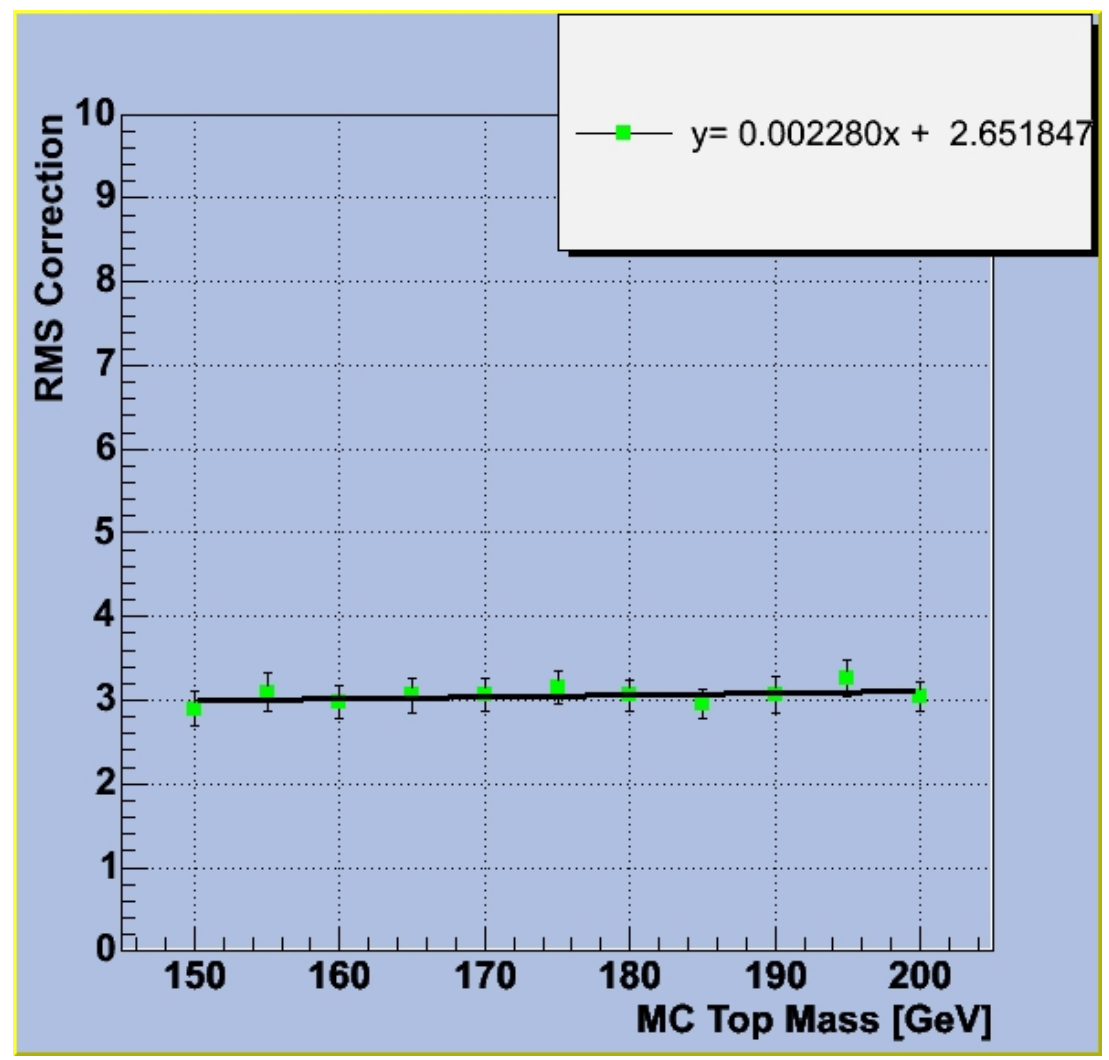

Figure 15: RMS correction factor as a function of top mass for signal-only events.

To begin to test the method, parton information from the MC truth tables are fed into it. This will test the method to see how well it does with the best possible data. Ideally, given that actual 4-momenta of the leptons and $b$-quarks are being used instead of the reconstructed leptons and jets, if a mass MC sample with $M_{t}$ of $175 \mathrm{GeV}$ is given to the method, it will return the same mass as its answer. Additionally, to start out, the method is allowed to use the truth table to determine which combination of leptons and $b$-quarks is the correct one. After each of the different mass MC samples are analyzed in this way, the mapping function should come out with a slope of approximately one. Figure 17 shows this mapping function. It is important to note that the event selection was still done on the reconstructed quantities. After the events were selected, only then were the truth table quantities used.

The next check to make with the truth tables is to look at the response from the flipped combinations only (see Figure 18). In this case, the slope should be less than one. Ideally, the flatter the mapping function the better. A small slope means that this method is not sensitive to the incorrect combination.

Lastly, the method is allowed to choose between the combinations itself based on the relative 
Figure 16: RMS correction factor as a function of top mass for signal and background events.

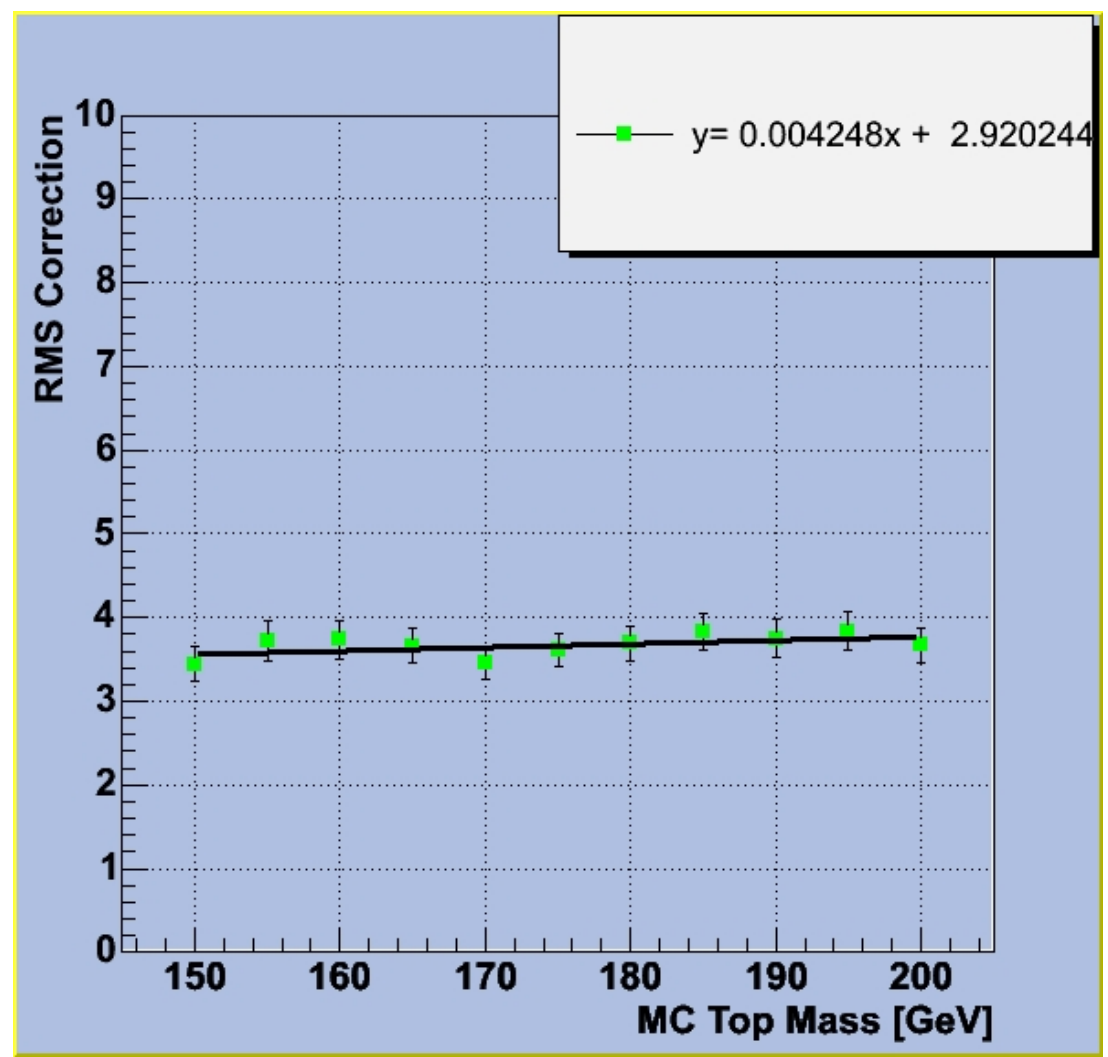

likelihood of each combination (See Figure 19) ${ }^{1}$. Given that the information comes from the truth tables, there are only two combinations, the correct one and the "flipped" combination.

\footnotetext{
${ }^{1}$ The error bars assigned to variables such as the reconstructed mass, residuals, pulls, and pull widths are determined by the bootstrap method. Appendix A gives an explanation of how it is done.
} 

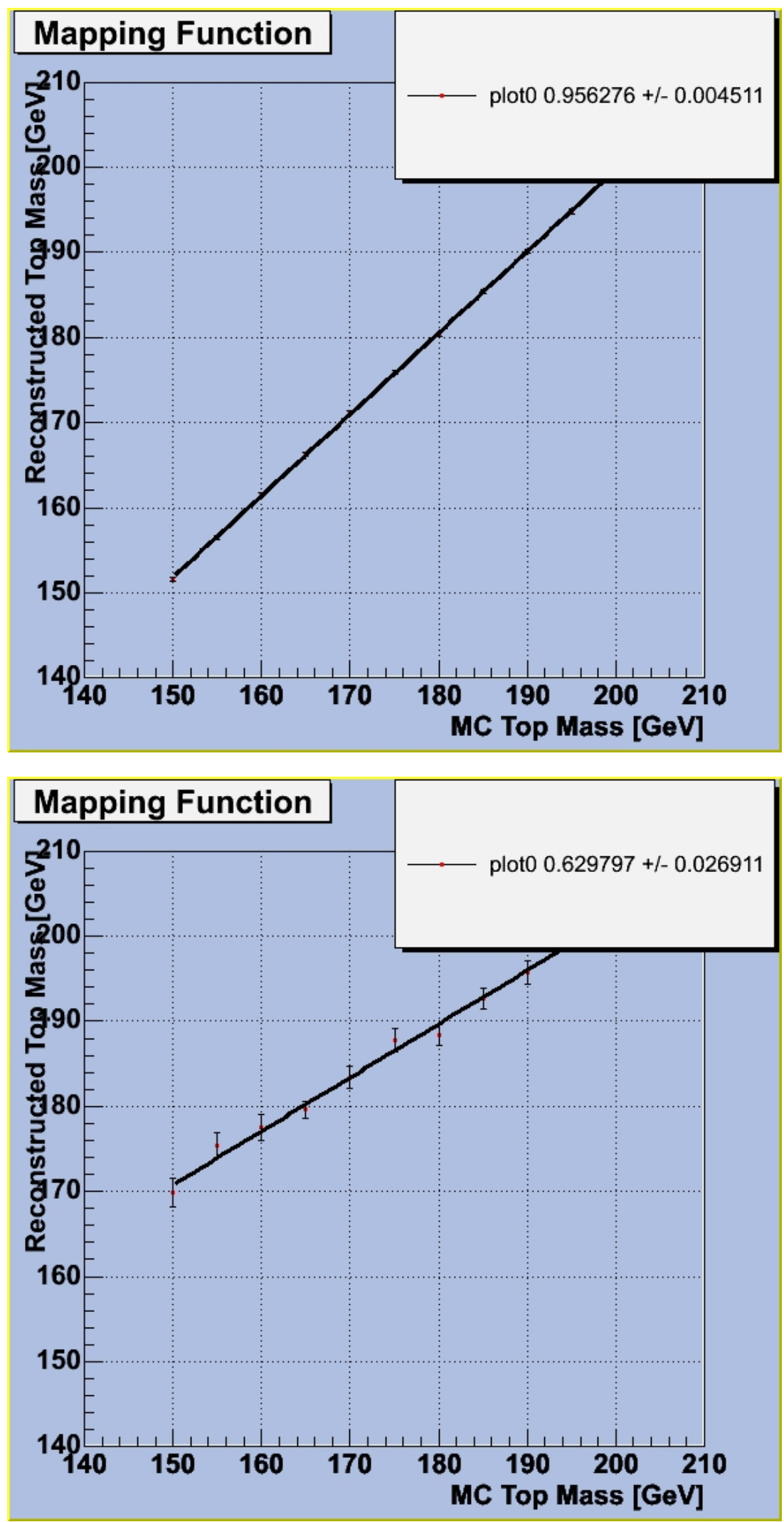

Figure 17: Mapping function from signal-only MC truth tables with only the correct combinations.
Figure 18: Mapping function from signal-only MC truth tables with the flipped combinations only. 

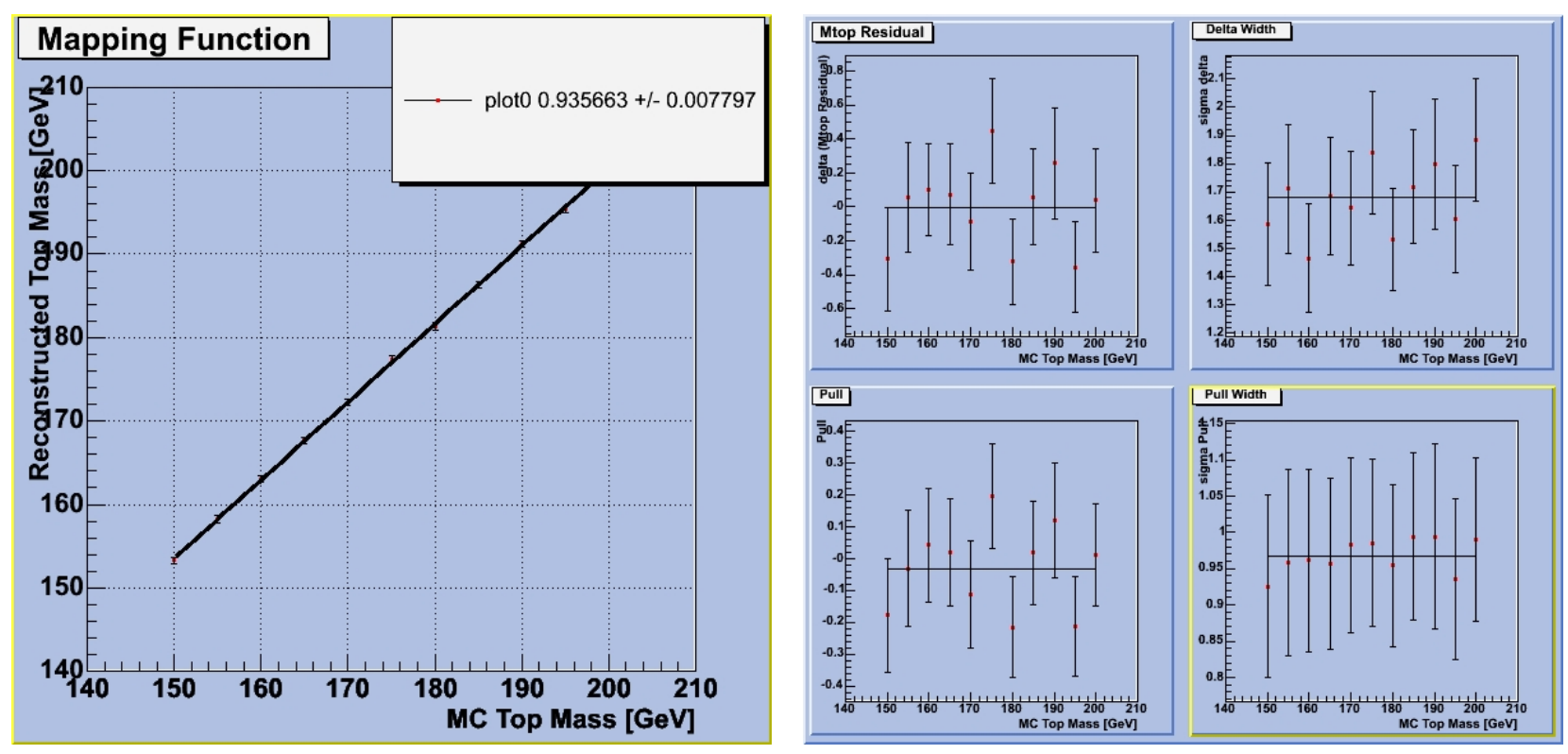

Figure 19: Mapping function from signal-only MC truth tables (Left). Delta, Delta Width, Pull, and Pull Widths for the signal MC truth table data samples after corrections have been applied from the mapping function (Right). 
The 'flipped' combination is the one where the two leptons and two $b$-quarks (jets) are mismatched, but are the correct four objects from the $t \bar{t}$ decay.

The next step in testing this method is to start to feed it some reconstructed MC data. Instead of giving it the 4-momenta from the actual leptons and $b$-quarks in the truth table, the reconstructed leptons and jets were used. The problem of correctly choosing the correct combination now becomes more challenging because an event may have more than just two jets. For testing purposes, the truth table information was kept so that the correct (Figure 20), flipped (Figure 21), and even "wrong" (Figure 22) combinations could be forced. The "wrong" combination is any combination where one or more of the four measurable decay products in the event did not correspond to one of the leptons or $b$-quarks in the $t \bar{t}$ decay in the truth table. For example, a combination where the two reconstructed leptons and one of the jets matched the truth table leptons and one of the $b$-quarks, but the other jet and remaining $b$-quark did not make a good match. Also, it is entirely possible and not rare that an event might not have a correct and wrong combination. Typically, this occurs when one of the two jets from the $t \bar{t}$ decay did not pass the tight jet cuts while a jet from some other process did. In this case,

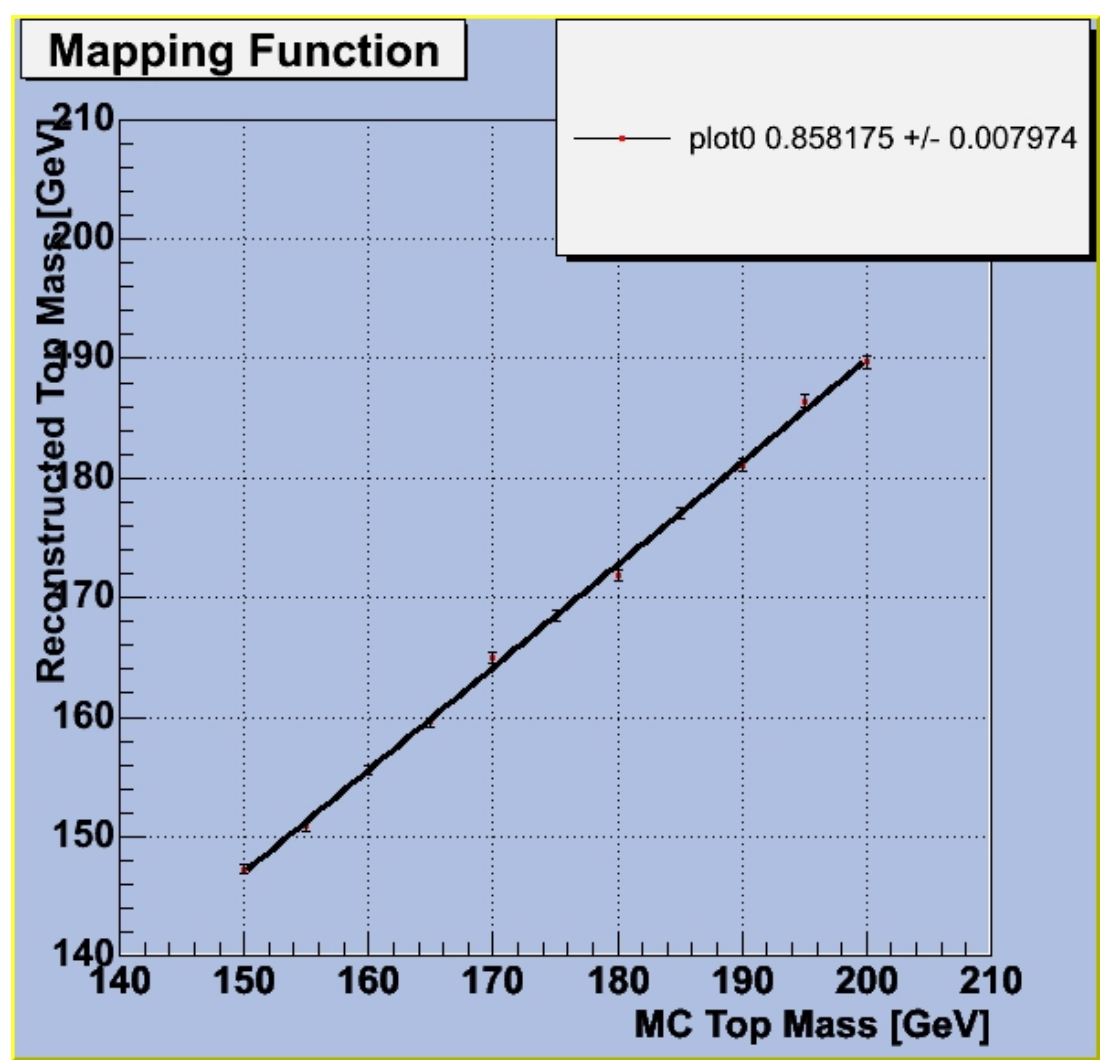

Figure 20: Mapping function from signal-only MC matchable events using only the correct combination. 
Figure 21: Mapping function from signal-only MC matchable events using only the flipped combination.

Figure 22: Mapping function from signal-only MC matchable events using only a wrong combination.
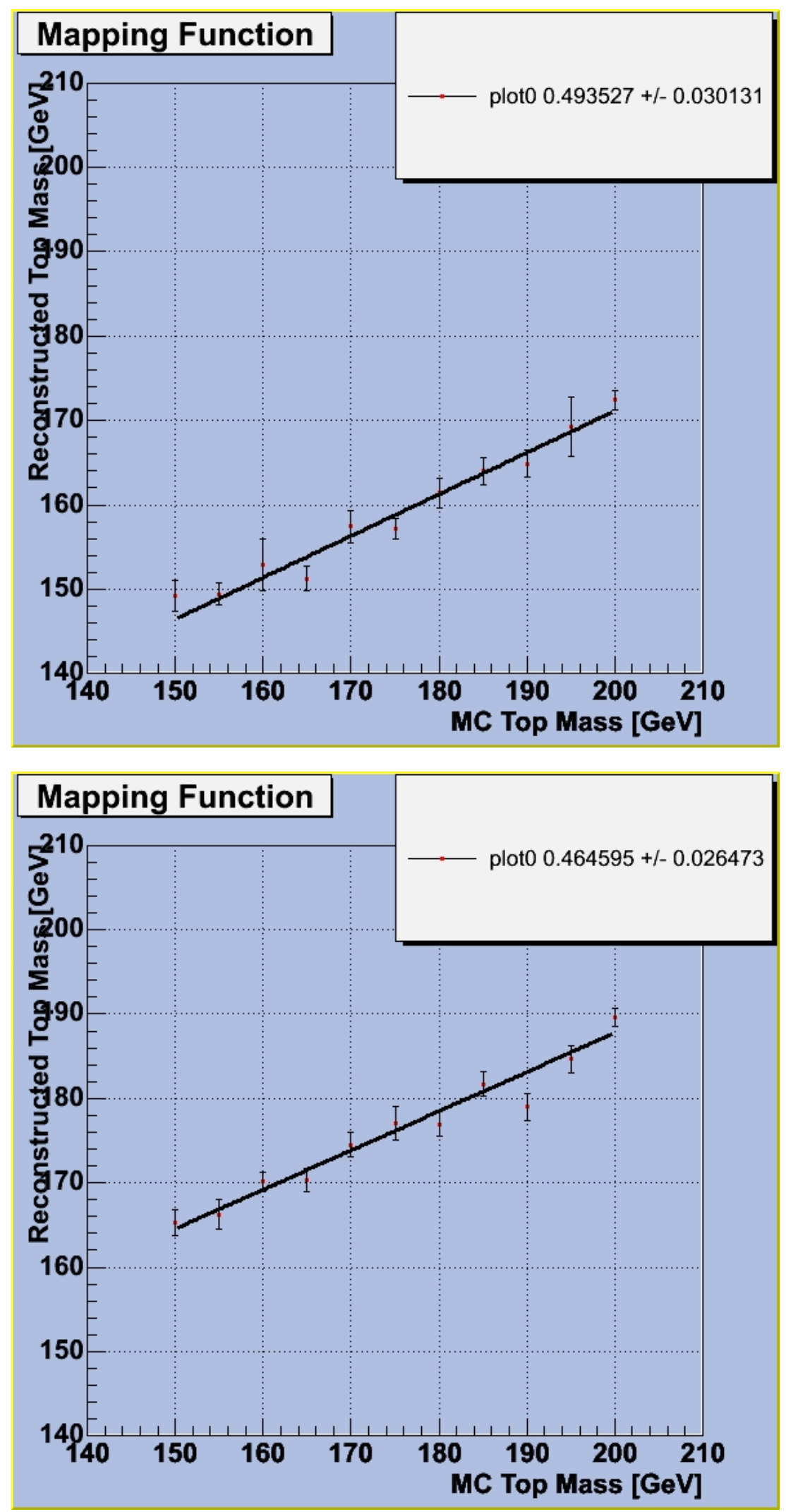
it is possible that the method will yield an answer, but answer will be false since some of its input was also false. There is no way to distinguish between this type of event and other good events without use of the truth tables. Nonetheless, the first run of tests with the reconstructed MC data on the method will use the truth table information to eliminate this type of event. The remaining events are considered to be "matchable." Figure 23 demonstrates how the method works with all matchable events where the combination of leptons and jets are chosen by their likelihood.
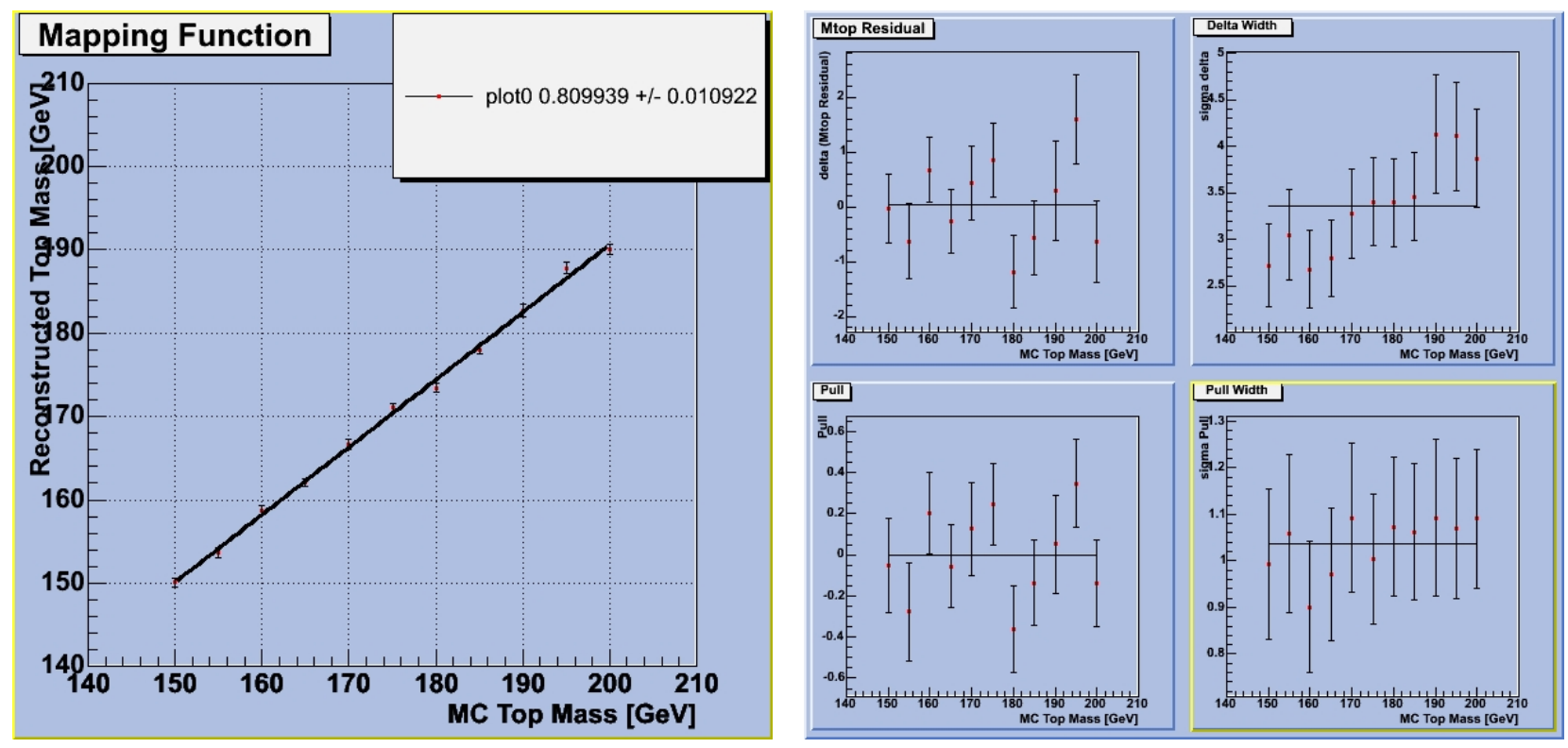

Figure 23: Mapping function from signal-only MC matchable events using a combination chosen by its likelihood (Left). Delta, Delta Width, Pull, and Pull Widths (Right).

While looking at the results from using only matchable events gives an idea of how well the method can work with reconstructed data, it is unrealistic. So, the next step is to look at all of the events that pass the di-lepton cuts. Figures 24 through 27 demonstrate how the method performs with all signal events, not just the ones classified as matchable. 
Figure 24: Mapping function from signal-only $\mathrm{MC}$ events using only the correct combination.
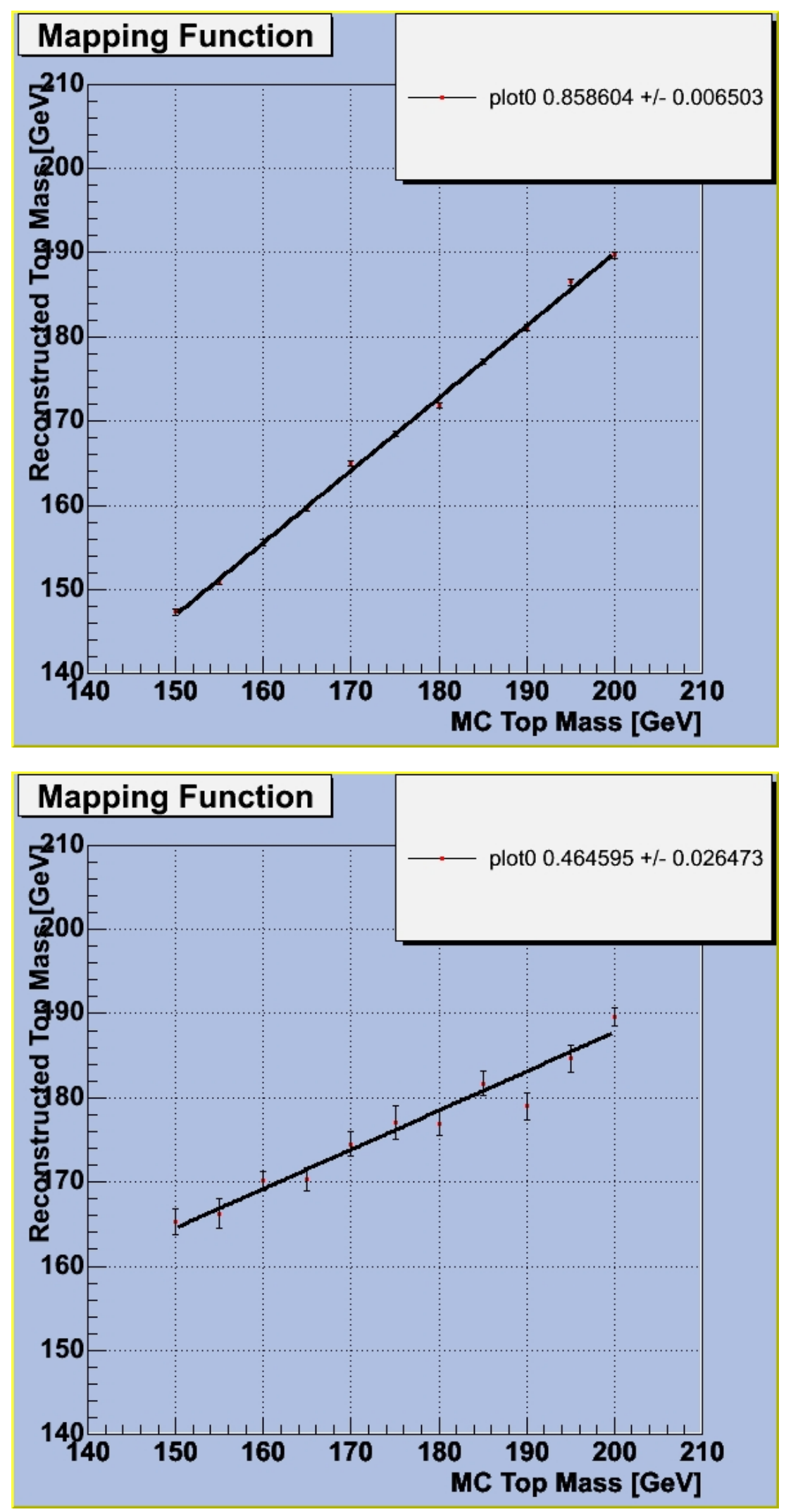

Figure 25: Mapping function from signal-only $\mathrm{MC}$ events using only the flipped combination. 


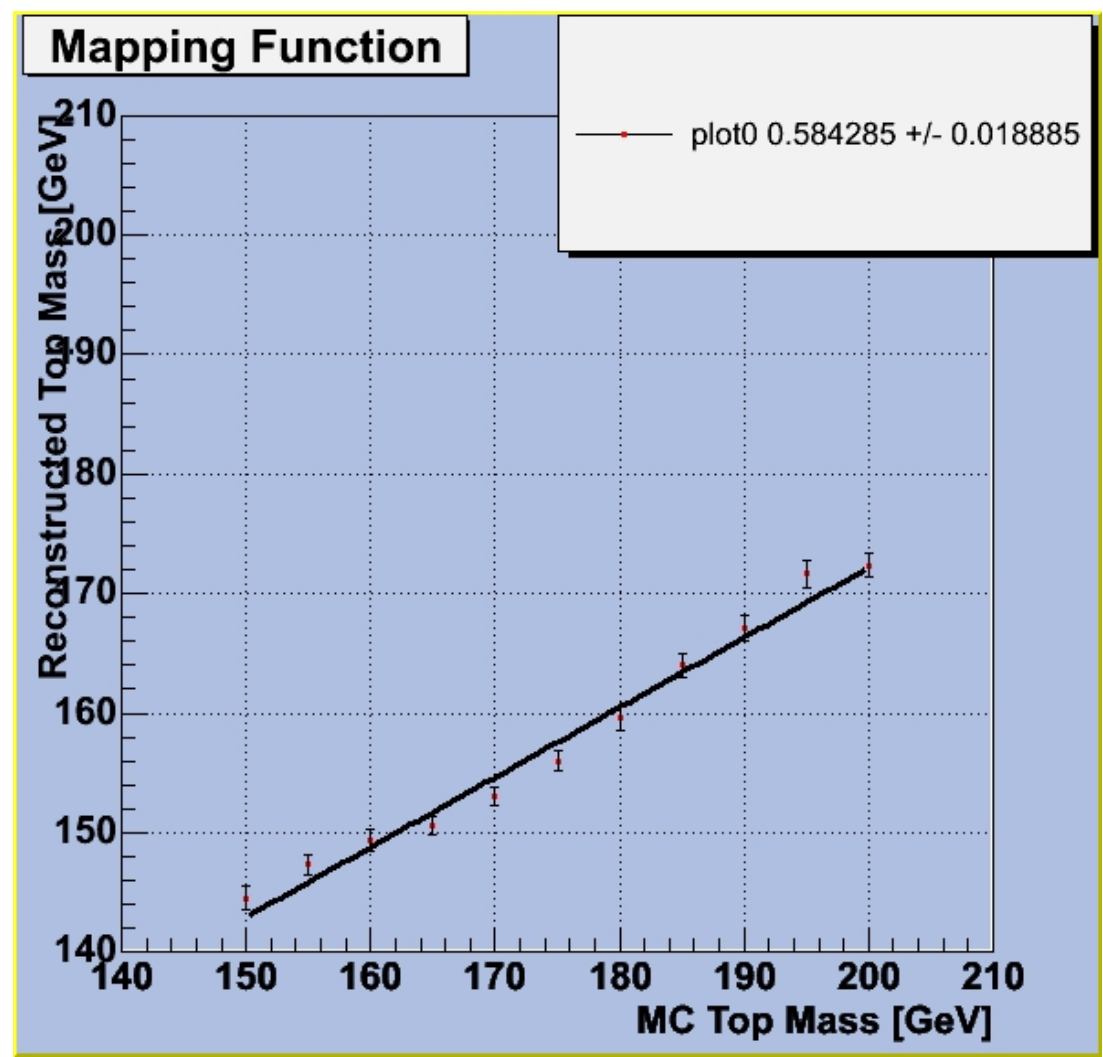

Figure 26: Mapping function from signal-only MC events using only a wrong combination.
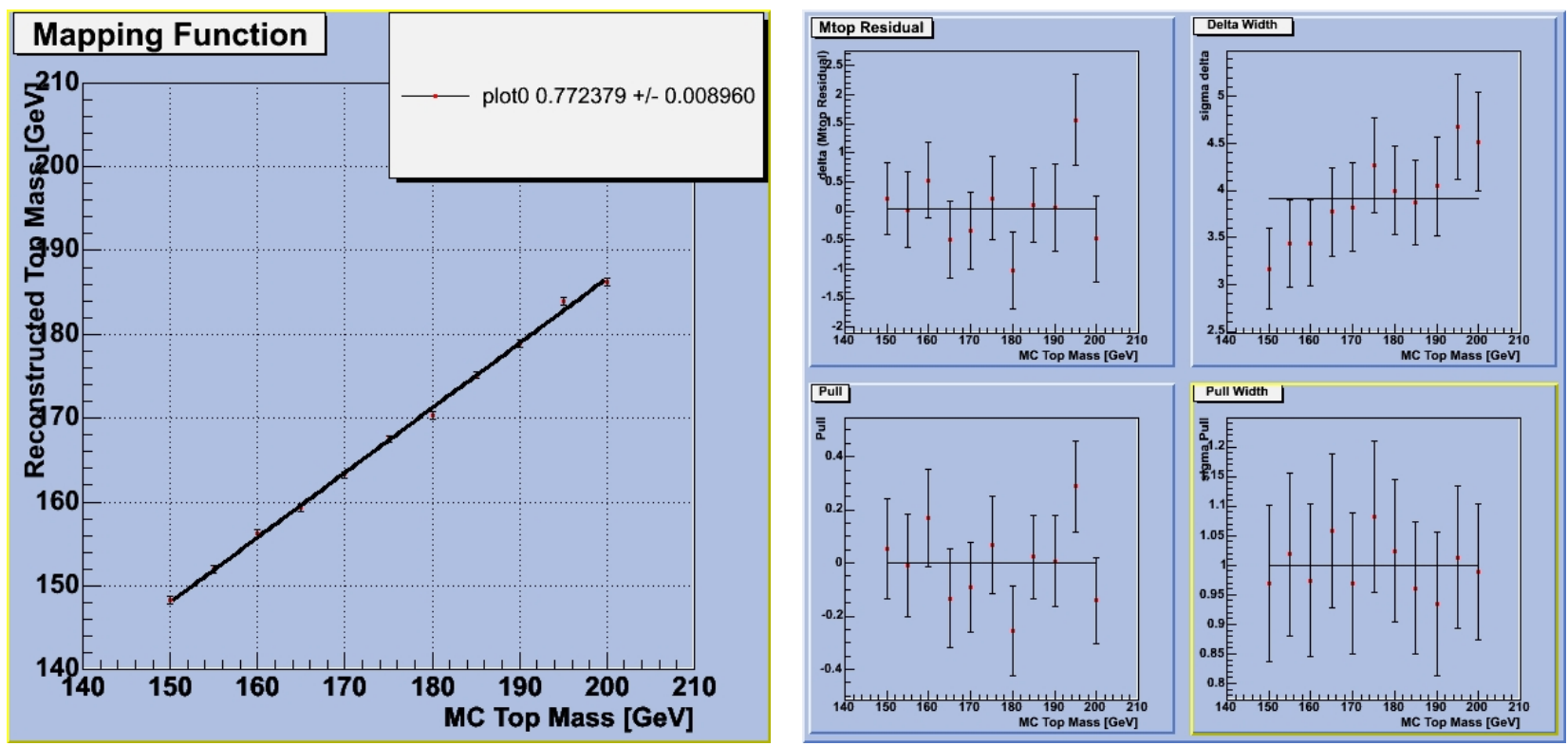

Figure 27: Mapping function from signal-only $\mathrm{MC}$ events using a combination chosen by its likelihood (Left). Delta, Delta Width, Pull, and Pull Widths (Right). 


\subsubsection{Additional Types of Combination Selection}

In addition to selecting a combination of leptons and jets according to their relative probabilities, three other methods are also studied. The first method is to combine both the most likely combination with its "twin" (Figure 28). The "twin" is simply the combination where the jetsleptons assignment is switched ${ }^{3}$. Both probability distributions are added together for each event before the pseudo-experiments are constructed. The second method is to select only the combinations in an event that have both of the two leading jets (Figure 29) - that is, the jets with the highest transverse energy. After the two combinations are found, the one with the highest probability is chosen. The final method differs from the second in that the two combinations that contain the two leading jets are added together (Figure 30), just like in the first additional method.
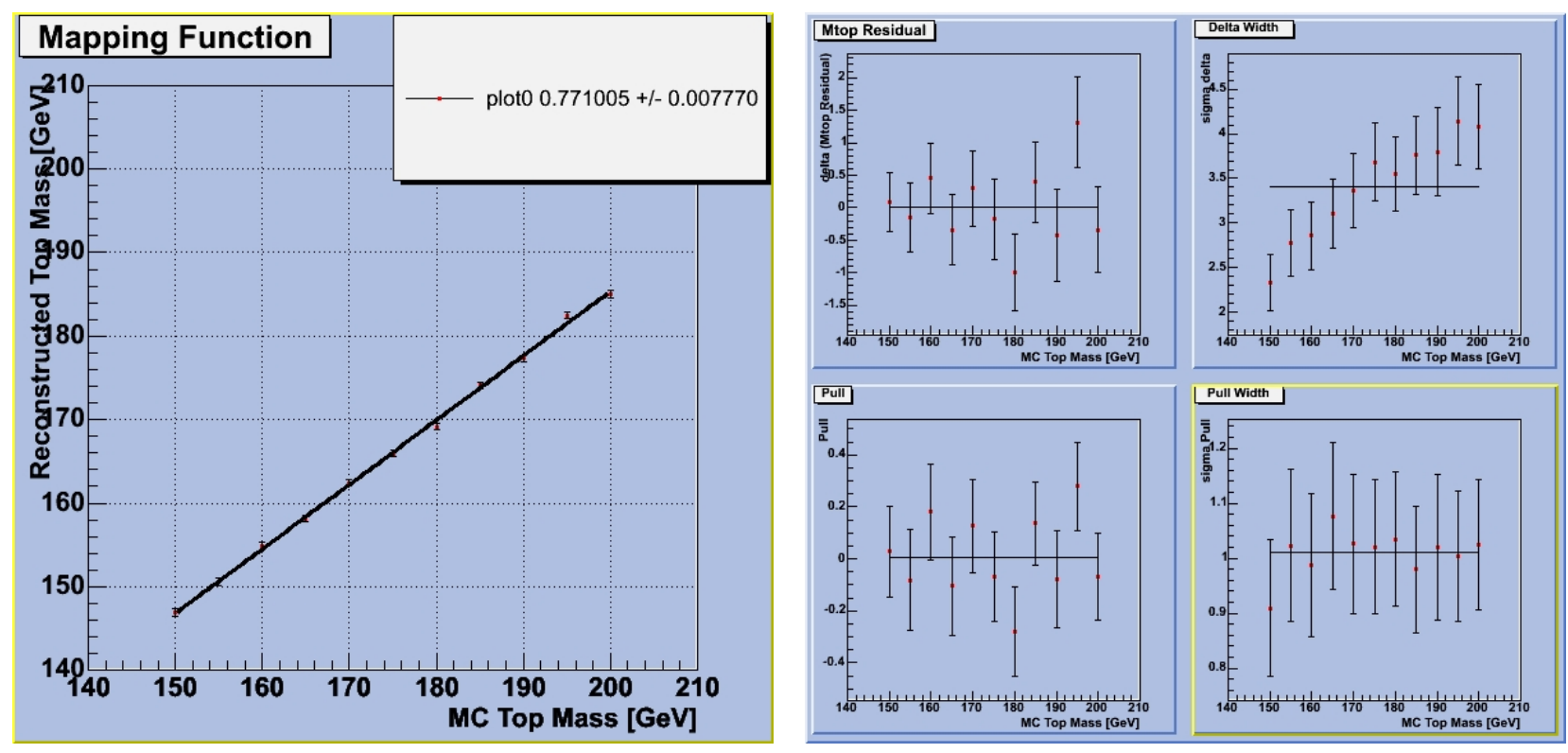

Figure 28: Mapping functions, Deltas, Delta Widths, Pulls, and Pull Widths for the signal MC data samples where the most probable combination is added to its "twin".

\footnotetext{
${ }^{3}$ There is an important distinction between the terms "twin" and "flipped" combination. Flipped denotes that there is knowledge from the truth table that says this combination has all of the correct decay object, but they are mismatched, while twin only designates a relation to another combination with the same four decay products.
} 

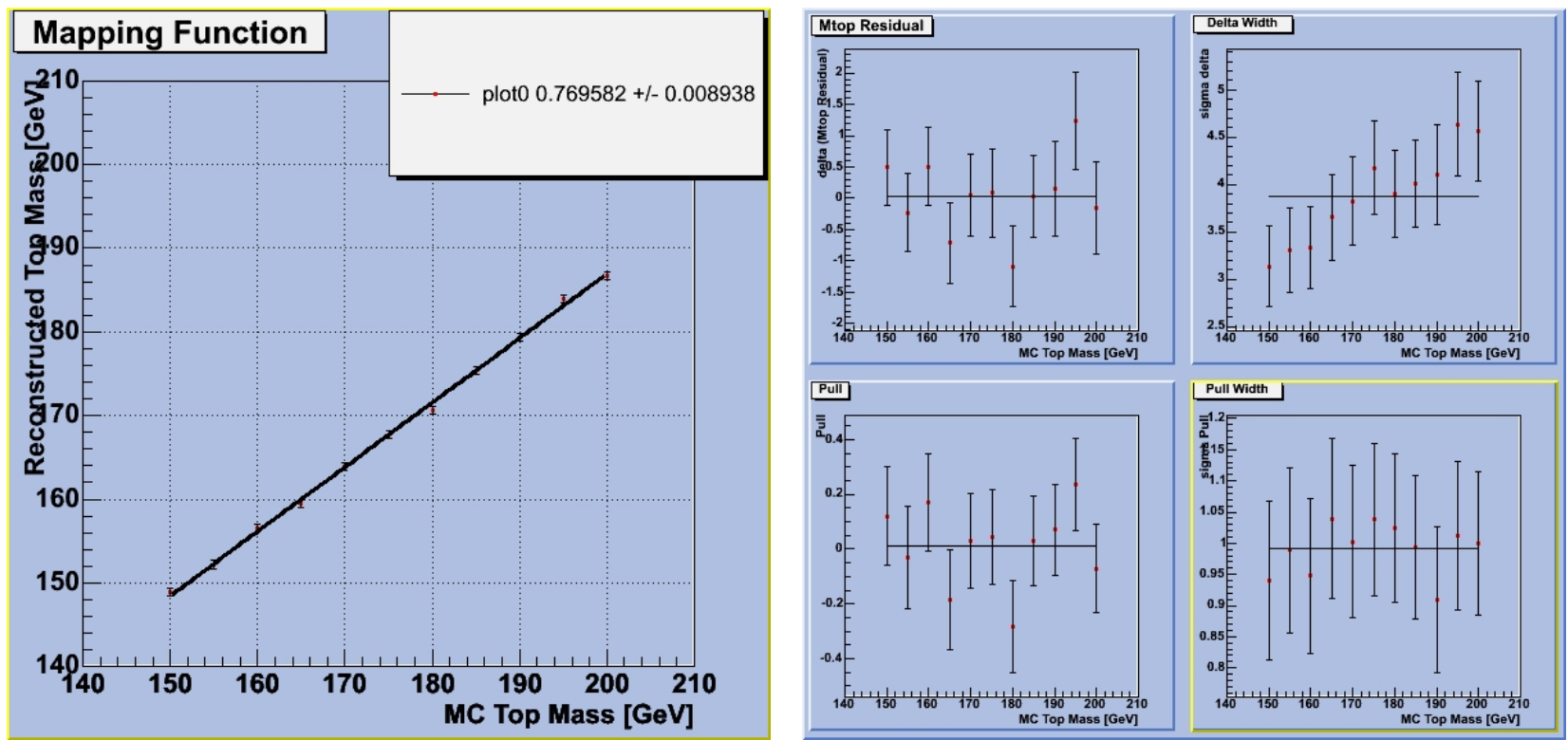

Figure 29: Mapping functions, Deltas, Delta Widths, Pulls, and Pull Widths for the signal MC data samples where the most probable combination is selected out of the combinations which contain both of the leading jets.
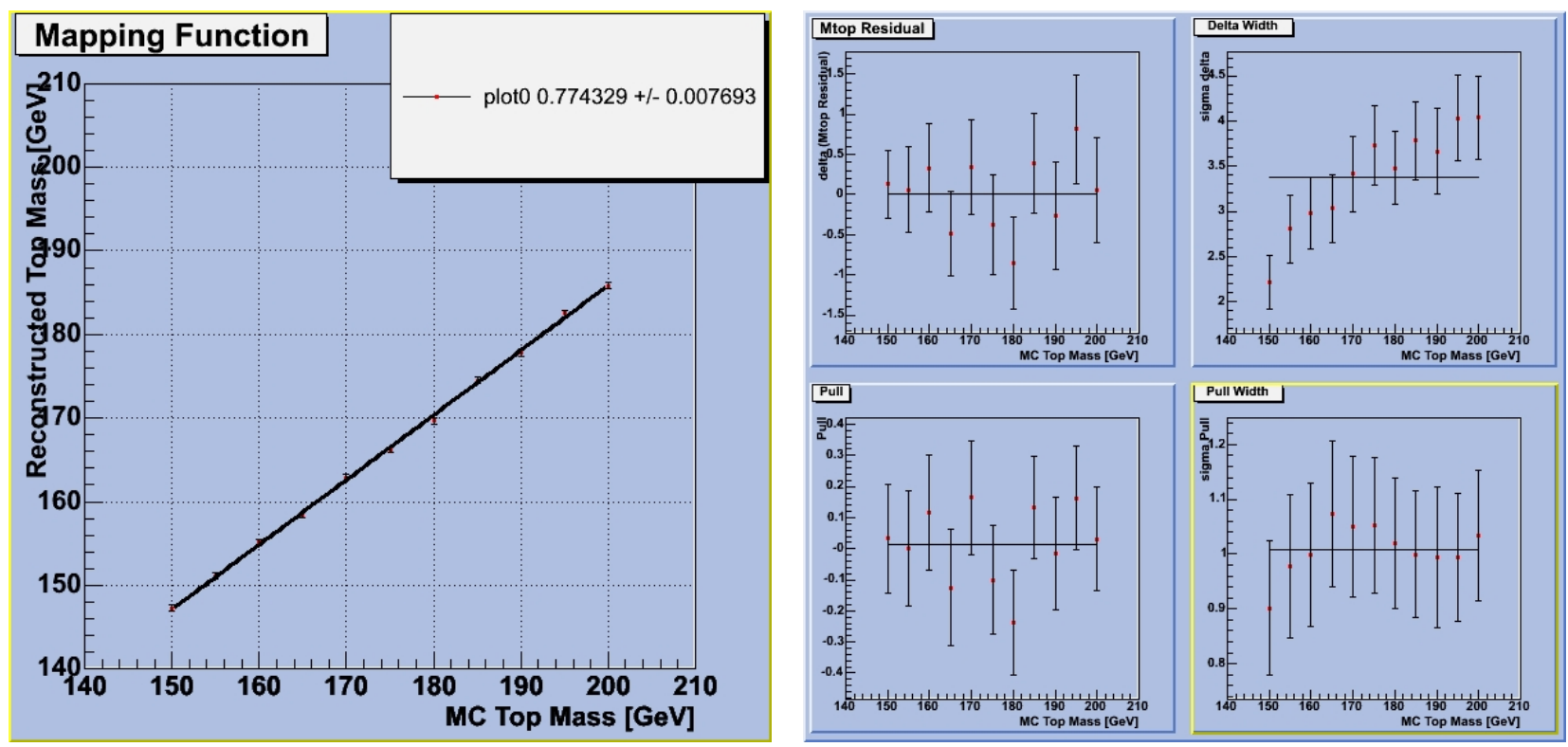

Figure 30: Mapping functions, Deltas, Delta Widths, Pulls, and Pull Widths for the signal MC data samples where the most probable combination is selected out of the combinations which contain both of the leading jets and is added to its "twin". 


\subsubsection{Contributions from Backgrounds}

Due to the presence of background events in the data, pseudo-experiments need to include both signal events and background events in order to find the proper mapping function that will be used to correct the raw mass from the real data. Pseudo-experiments are constructed in the same way as described in section 5.6 of this chapter, except that now the signal and each type of background forms its own pool of events. Events are randomly selected from each pool in proportion to the number of expected events for each background and the signal. See Table 13 for the number of expected events for the signal and backgrounds. Instead of fluctuating the total number of events in a pseudo-experiment, the number of expected signal events and the total number of expected background events are both individually fluctuated using a Poisson distribution. The number of events for each individual background is kept in proportion based on the fluctuated value for the total number of background events.

Figures 31 through 34 show the results from the addition of background to the pseudoexperiments.
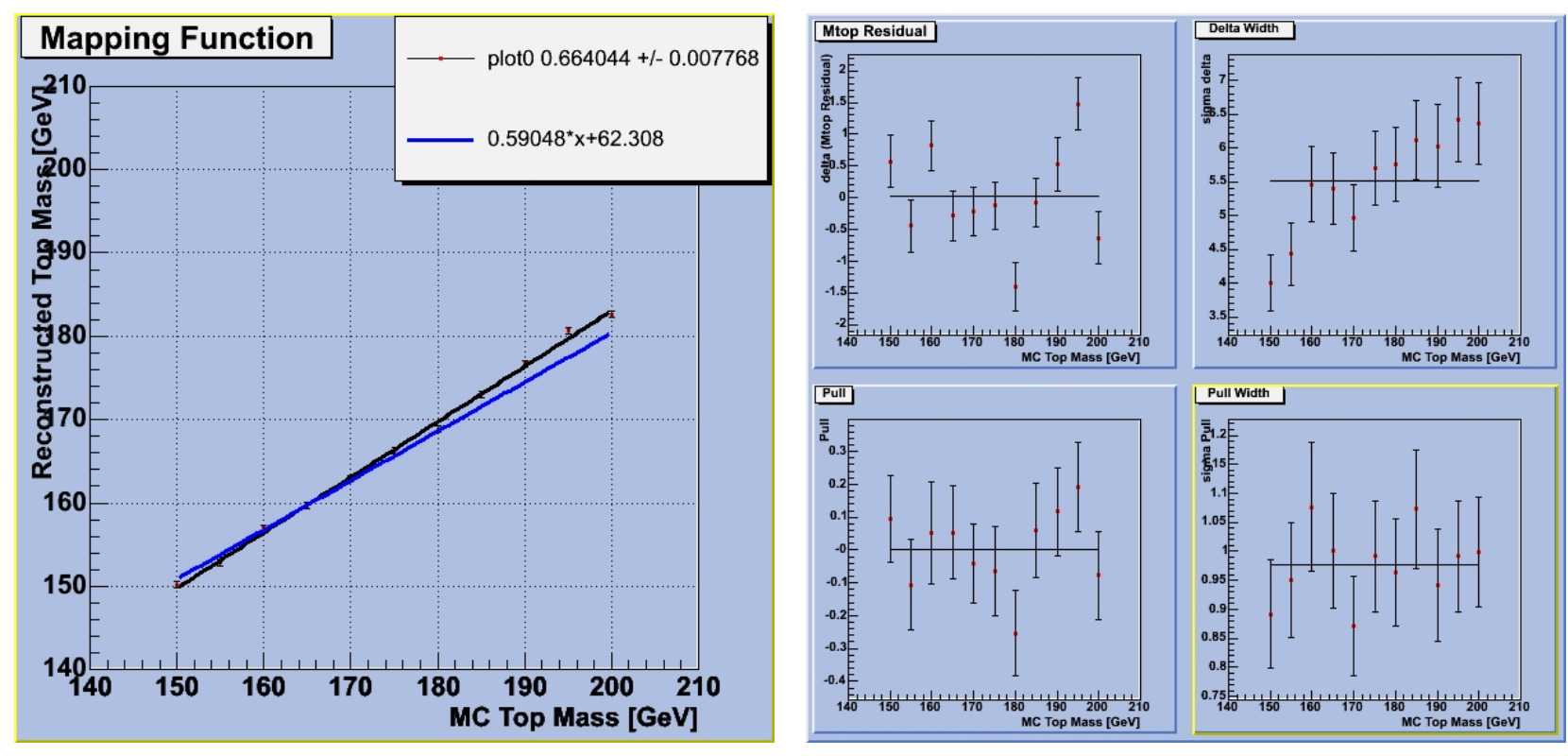

Figure 31: Mapping functions, Deltas, Delta Widths, Pulls, and Pull Widths for the signal and background data samples where the most probable combination is selected. The blue line is the mapping function used by Karr and Sliwa[7]. 

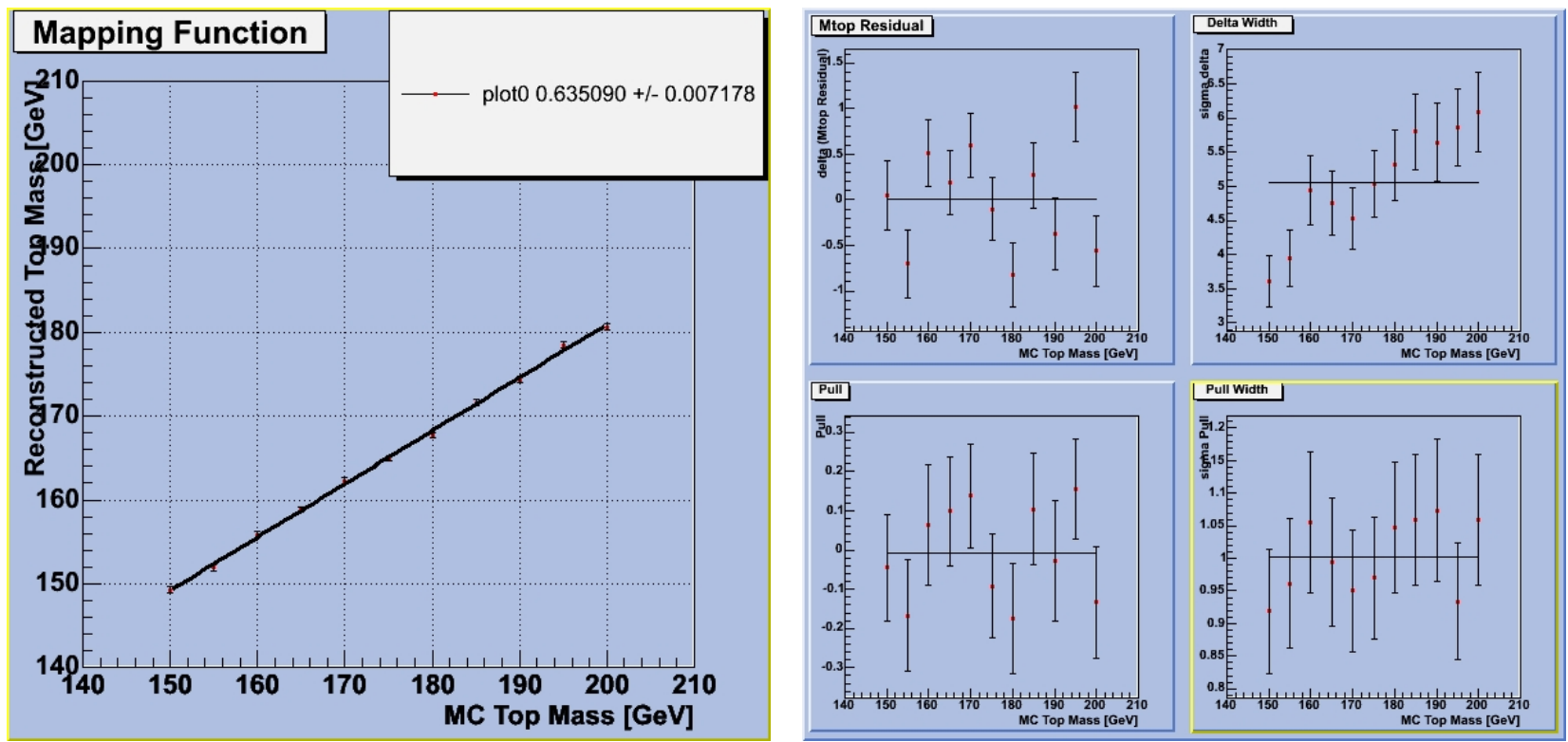

Figure 32: Mapping functions, Deltas, Delta Widths, Pulls, and Pull Widths for the signal and background data samples where the most probable combination is added to its "twin".
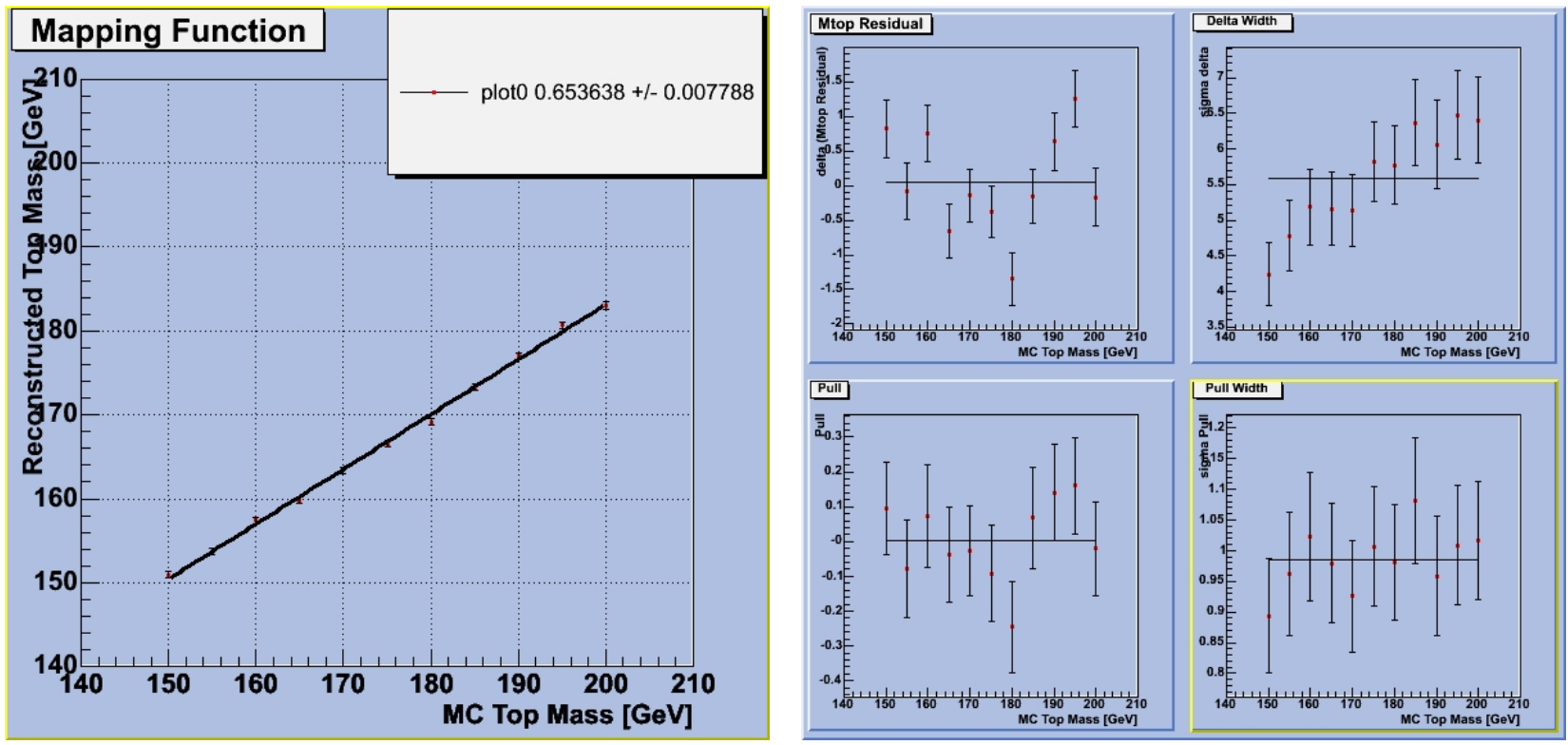

Figure 33: Mapping functions, Deltas, Delta Widths, Pulls, and Pull Widths for the signal and background data samples where the most probable combination is selected out of the combinations which contain both of the leading jets. 

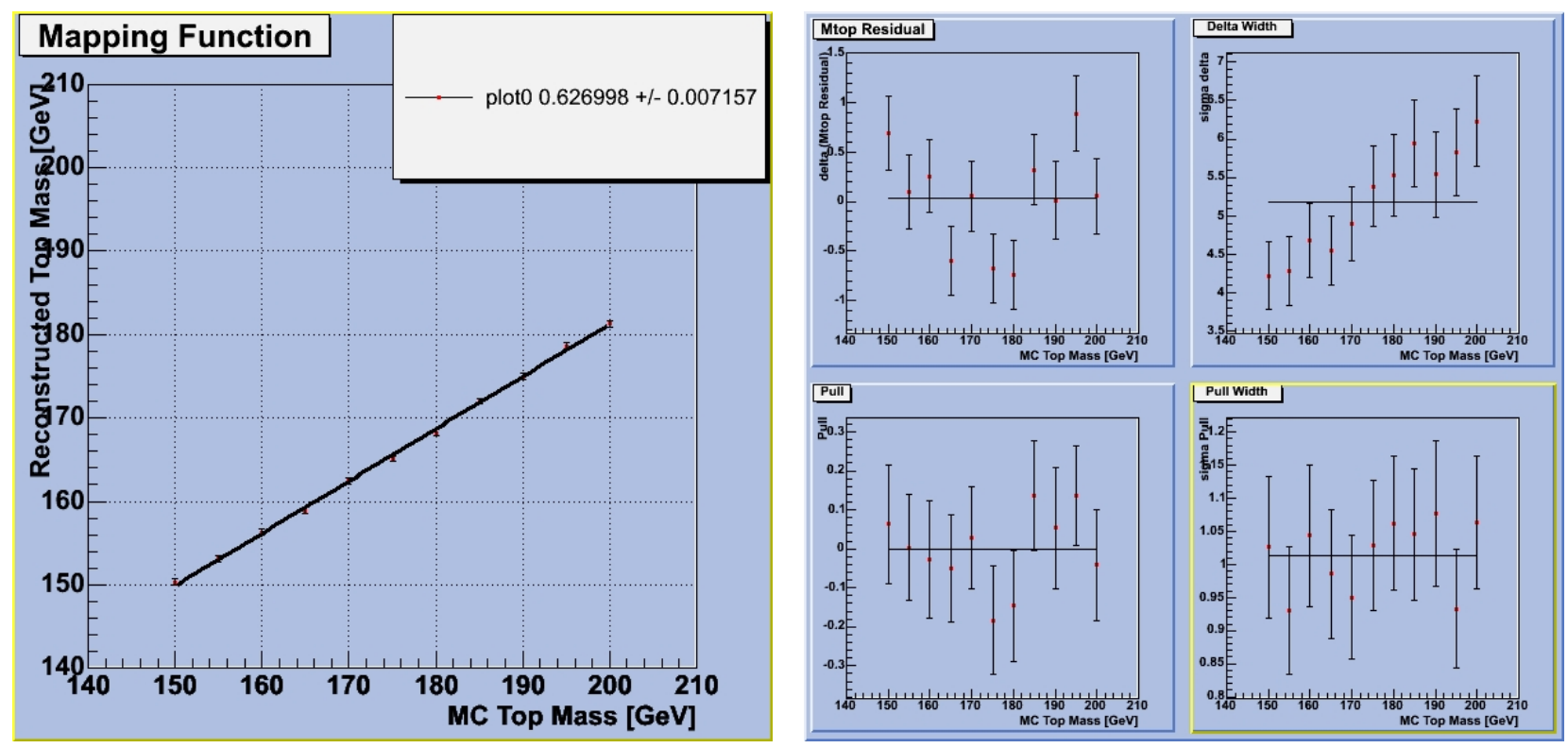

Figure 34: Mapping functions, Deltas, Delta Widths, Pulls, and Pull Widths for the signal and background data samples where the most probable combination is selected out of the combinations which contain both of the leading jets and is added to its "twin".

The four mapping functions that will be used to make the correction to the raw top mass are:

$$
\begin{array}{lc}
M_{t}^{\text {raw }}=0.665 \times M_{t}^{\text {corr }}+50.02 & \text { (Likelihood Only }) \\
M_{t}^{\text {raw }}=0.635 \times M_{t}^{\text {corr }}+53.95 & \text { (Likelihood Added }) \\
M_{t}^{\text {raw }}=0.654 \times M_{t}^{\text {corr }}+52.41 & (2 \text { Leading Jets }) \\
M_{t}^{\text {raw }}=0.627 \times M_{t}^{\text {corr }}+55.85 & (2 \text { Leading Jets Added })
\end{array}
$$




\section{7 $\quad$ Statistical Error}

The statistical error on the measurement of the top quark mass is expected to be related to the width of the joint distribution created by the product of the mass distributions from the real data events. In addition to the correction to the RMS as described in Section 5.6.1, the width of the joint distribution needs to be corrected using the mapping function for the top mass correction. This correction is simply the slope of the mapping function, $m_{\text {map }}$. The statistical error on the measurement of the top quark mass, $\sigma_{\text {stat }}^{\text {corr }}$ is related to the raw width of the joint distribution, $\sigma_{J D}^{r a w}$ by:

$$
\sigma_{\text {stat }}^{\text {corr }}=C_{r m s} \times \sigma_{J D}^{r a w} / m_{m a p}
$$

where $C_{r m s}$ is the RMS correction factor. The slopes and RMS correction factors are listed in Table 15 .

\begin{tabular}{lcc}
\hline Combination Choice & Slope & RMS Correction \\
\hline \hline Likelihood Only & 0.665 & 3.66 \\
Likelihood Added & 0.635 & 3.01 \\
2 Leading Jets & 0.654 & 3.61 \\
2 Leading Jets Added & 0.627 & 3.01 \\
\hline
\end{tabular}

Table 15: Correction factors to the width of the top mass joint distribution.

Figure 35 shows all of the correction factors from each data sample (the truth table, signalonly, and signal + background) and each method of choosing a combination from an event. The value of the correction factor appears to be related to the shape of the individual event probability distributions and the variation of the means of the individual events. The probability distributions that result from the truth table information tend to be much more symmetrical and smoother, somewhat more Gaussian in shape, than events that use the reconstructed quantities. Additionally, the spread of the means of a given sample is lower when using the truth table than the reconstructed quantities. Together, these effects create the need for a larger correction factor. Its important to note that no matter which type of data is used, the correction factors do not show a significant dependence on the mass of the top quark (See Figures 15 and 16).

Figure 36 shows the distributions of errors that can be expected from each of the four strategies of combining jets with leptons after corrections in this analysis based on the corrected 
RMS Correction Factors

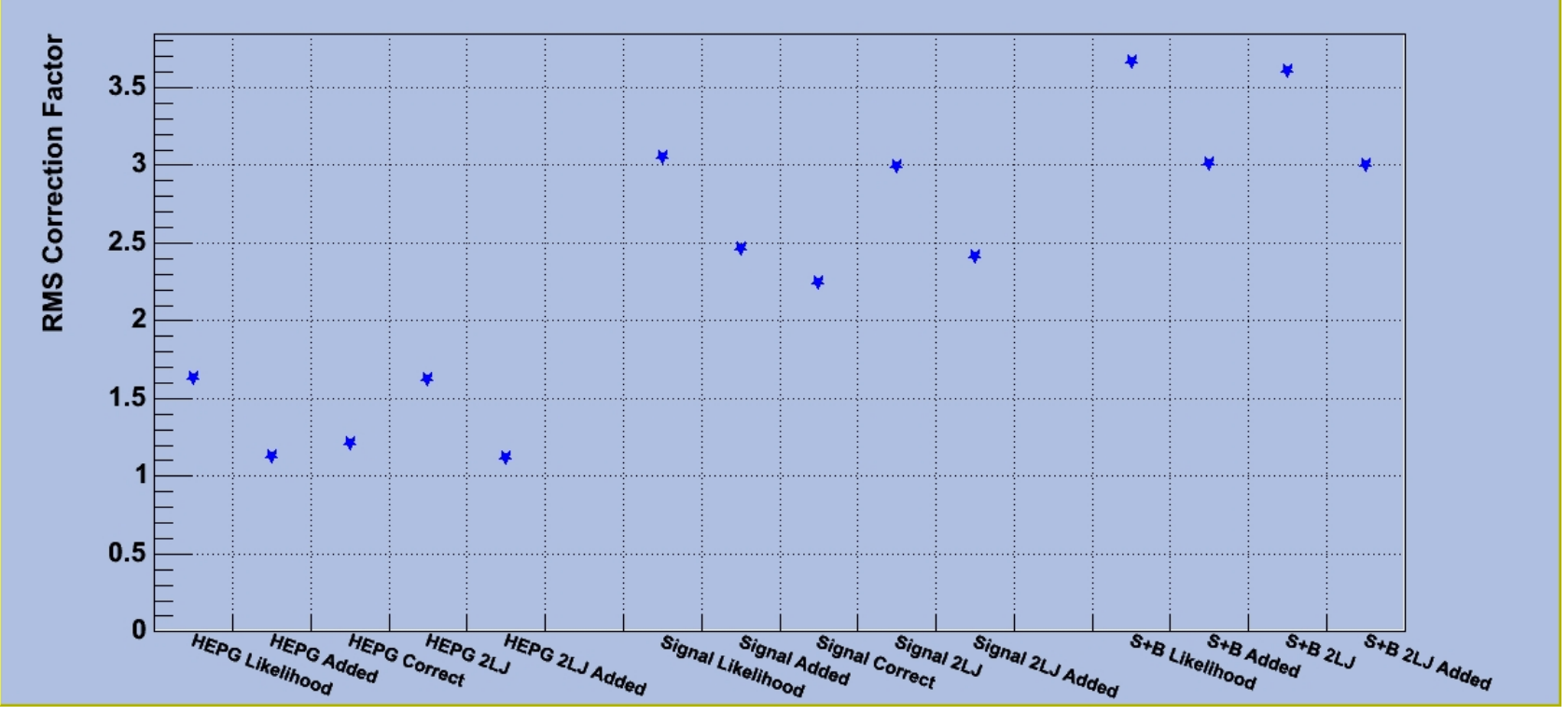

Figure 35: The various correction factors to the RMS by input sample and methodology.

widths of individual PEs. These distributions are based on MC data with $M_{t}$ set to $175 \mathrm{GeV}$. An independent way to estimate the expected statistical error on the mass measurement is to use the width of the top mass histogram that was filled with the masses of each of the PEs. The width of this histogram is the average error for the PEs and should also be the expected error on the measurement conducted with the real data. Figure 37 shows the errors as a function of $M_{t}$. Since this is the expected statistical error, it would be reasonable to use these plots to determine the error on the measurement once the value of $M_{t}$ was determined. However, does not allow for statistical variations in the real data since it is based on PEs from MC data. 

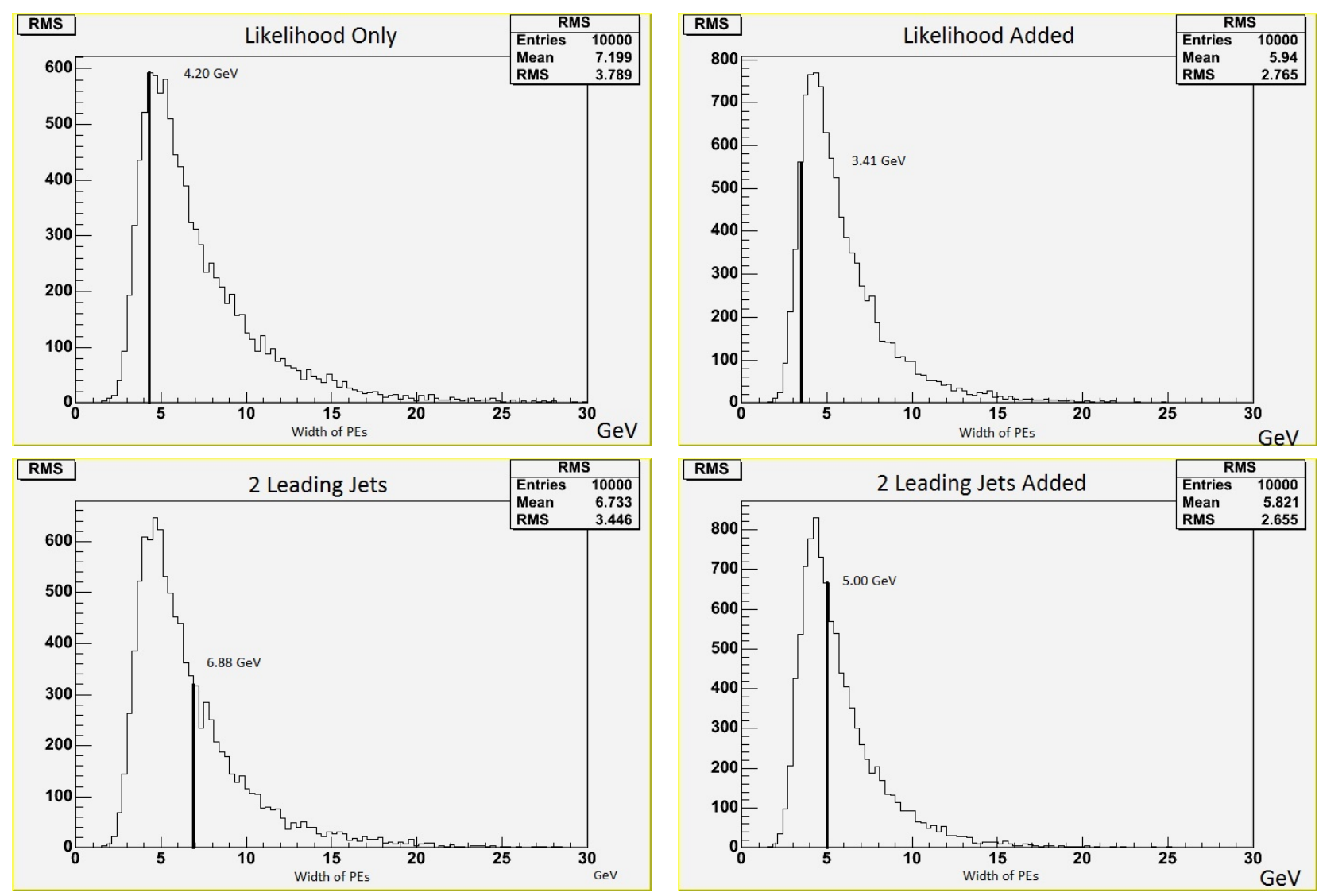

Figure 36: Distributions of corrected widths from the PEs for each of the combination strategies. Upper left is likelihood only, upper right is likelihood added, lower left is 2 leading jets chosen by likelihood, and lower right is 2 leading jets added together. The vertical line and value in each plot is the corresponding statistical error from the measurements. 

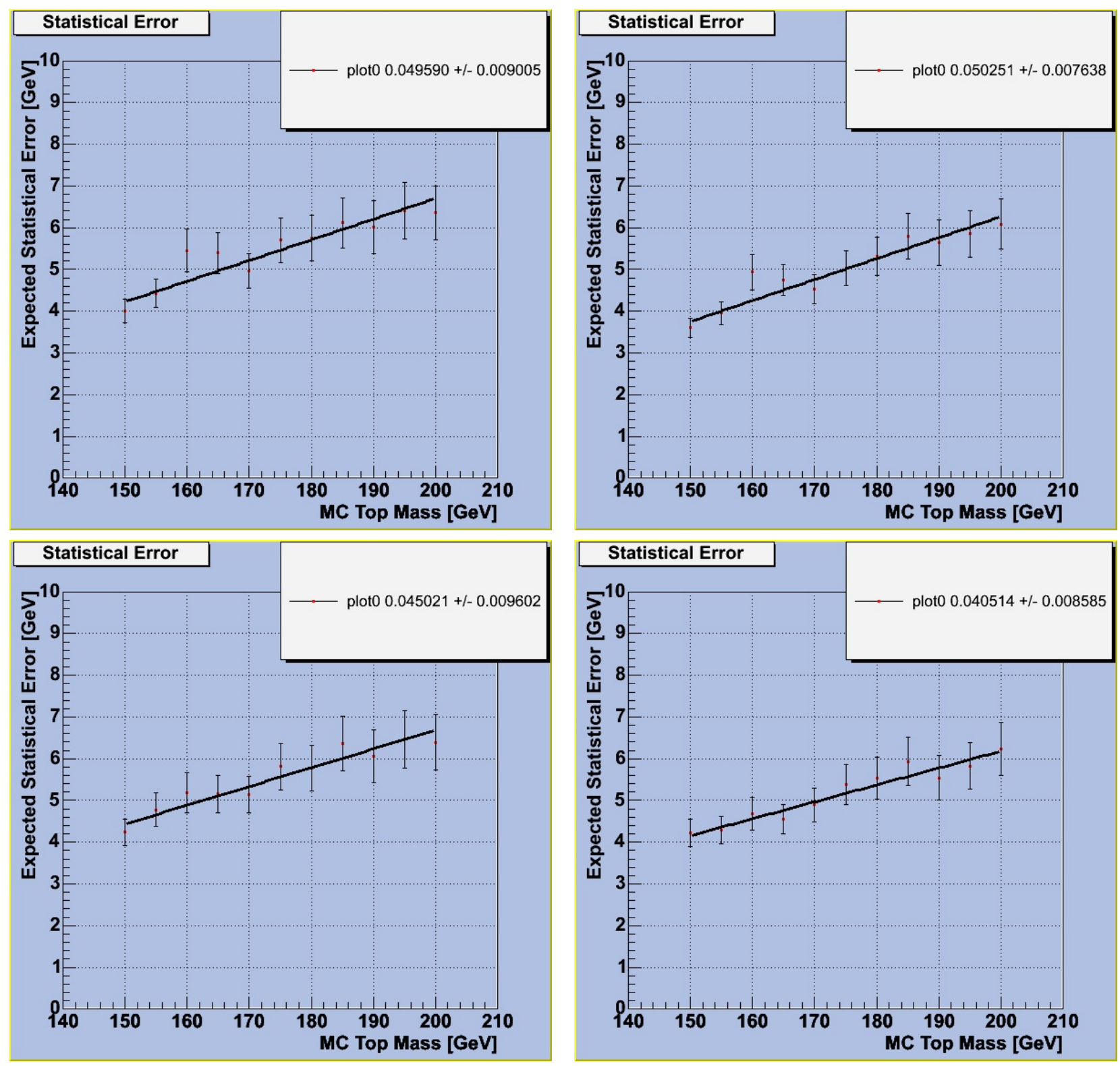

Figure 37: Expected statistical errors for each of the four combination strategies based on the width of the PE mass histogram. Upper left is likelihood only, upper right is likelihood added, lower left is 2 leading jets chosen by likelihood, and lower right is 2 leading jets added together. 


\subsection{Sanity Checks}

Before it is time to analyze the real data, it is important to have a closer look at how the method affects various variables. If a variable from the signal and background MC data does not match well with the variable in the real data, this would suggest that there is a problem in the method or that the variable is not being modeled well in the MC. The variables of interest are shown in Figures 38 through 42 and include such things like the $P_{t}, \eta$, and $\phi$ for each of the top quarks, the $t \bar{t}$-system, and each of the jets and leptons. Figures 38 through 42 show the variables most important to this analysis.
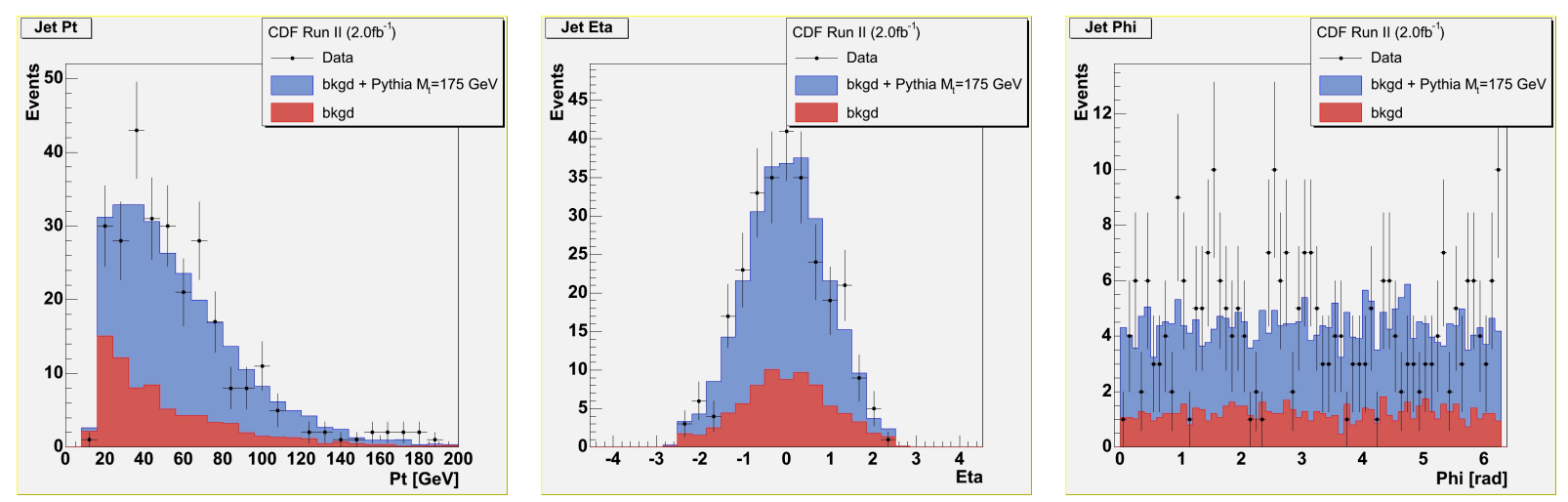

Figure 38: The $P_{t}, \eta$, and $\phi$ for the jets in both the MC data and the real data.
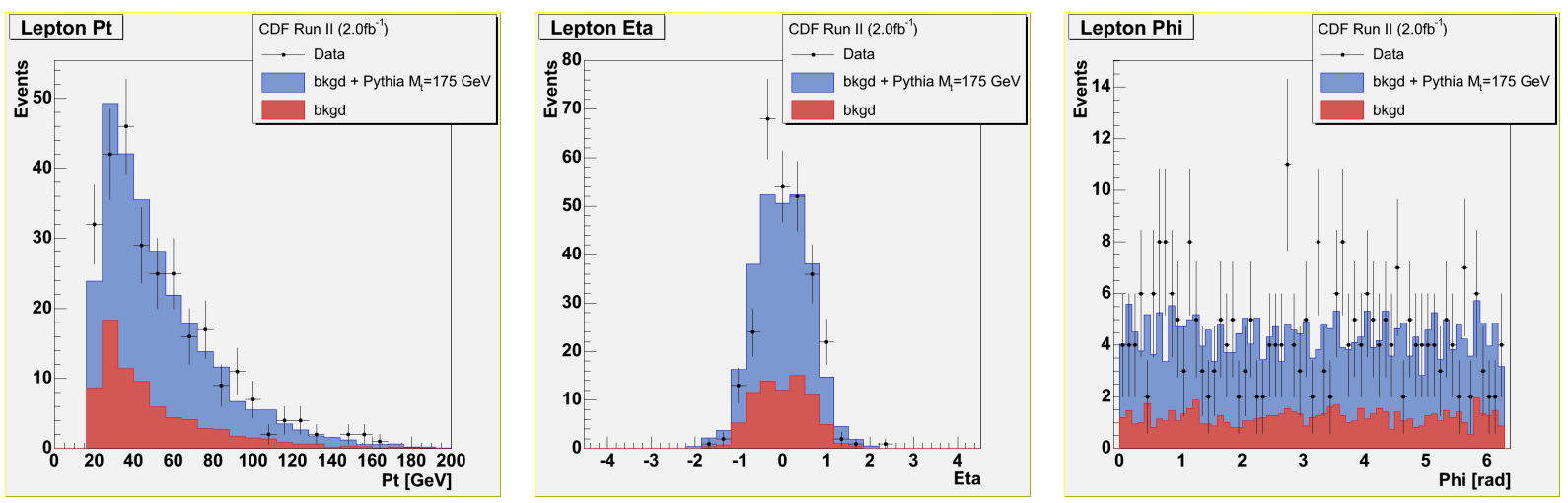

Figure 39: The $P_{t}, \eta$, and $\phi$ for the leptons in both the MC data and the real data. 

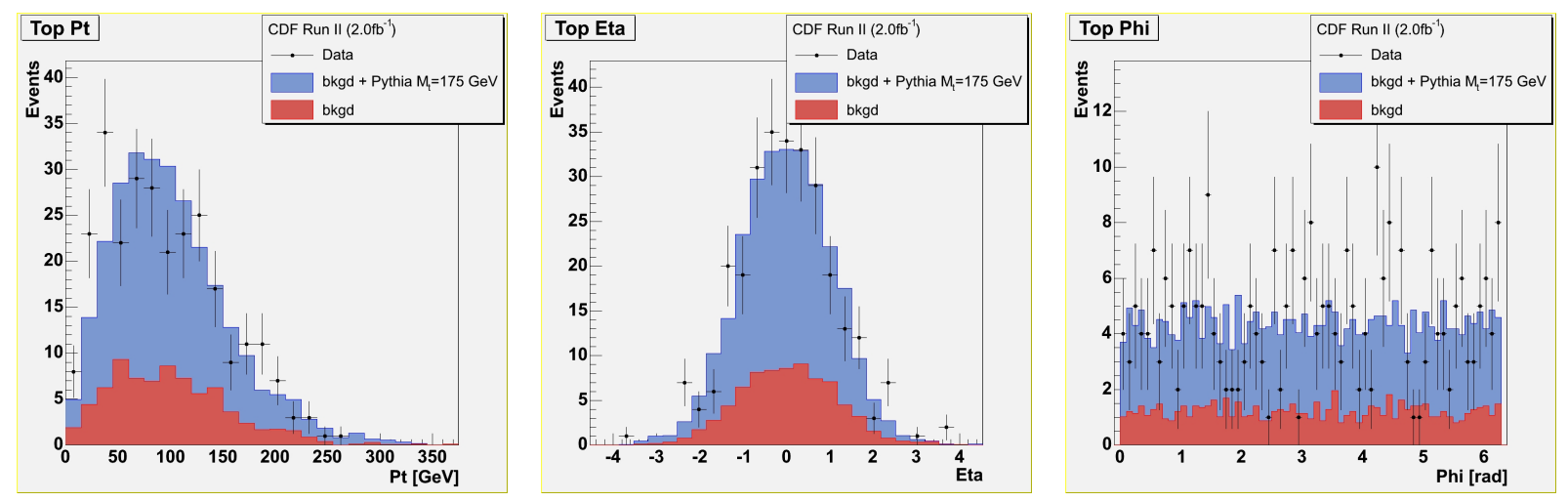

Figure 40: The $P_{t}, \eta$, and $\phi$ for the top quarks in both the MC data and the real data.
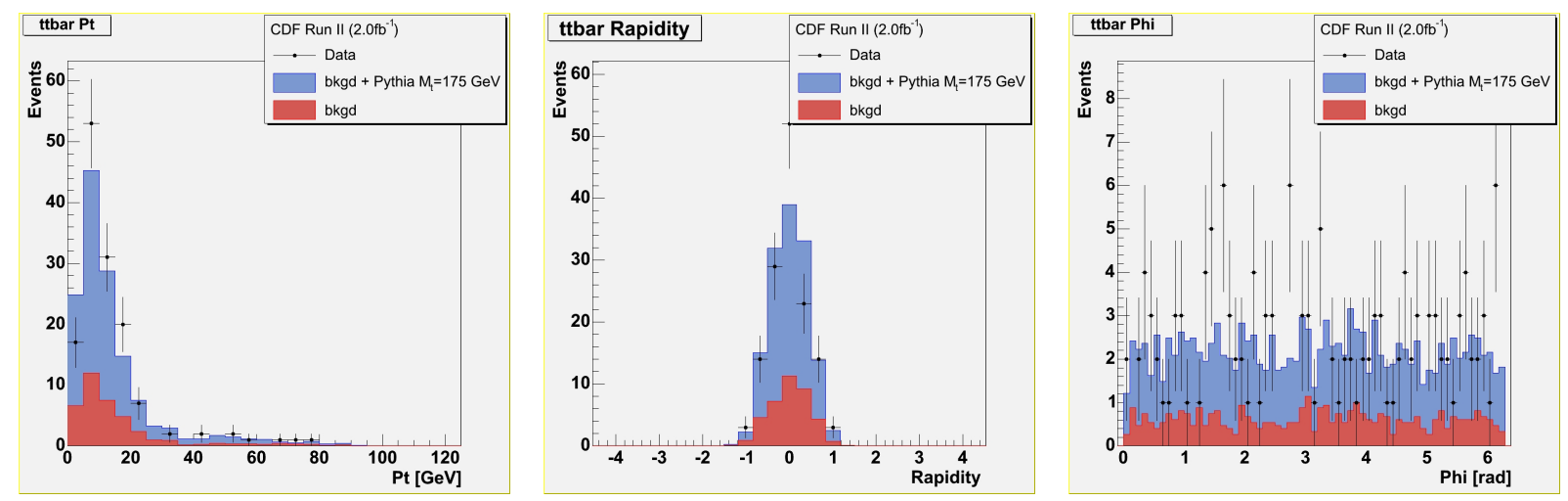

Figure 41: The $P_{t}$, rapidity, and $\phi$ for the top-anti-top quark system in both the MC data and the real data. 

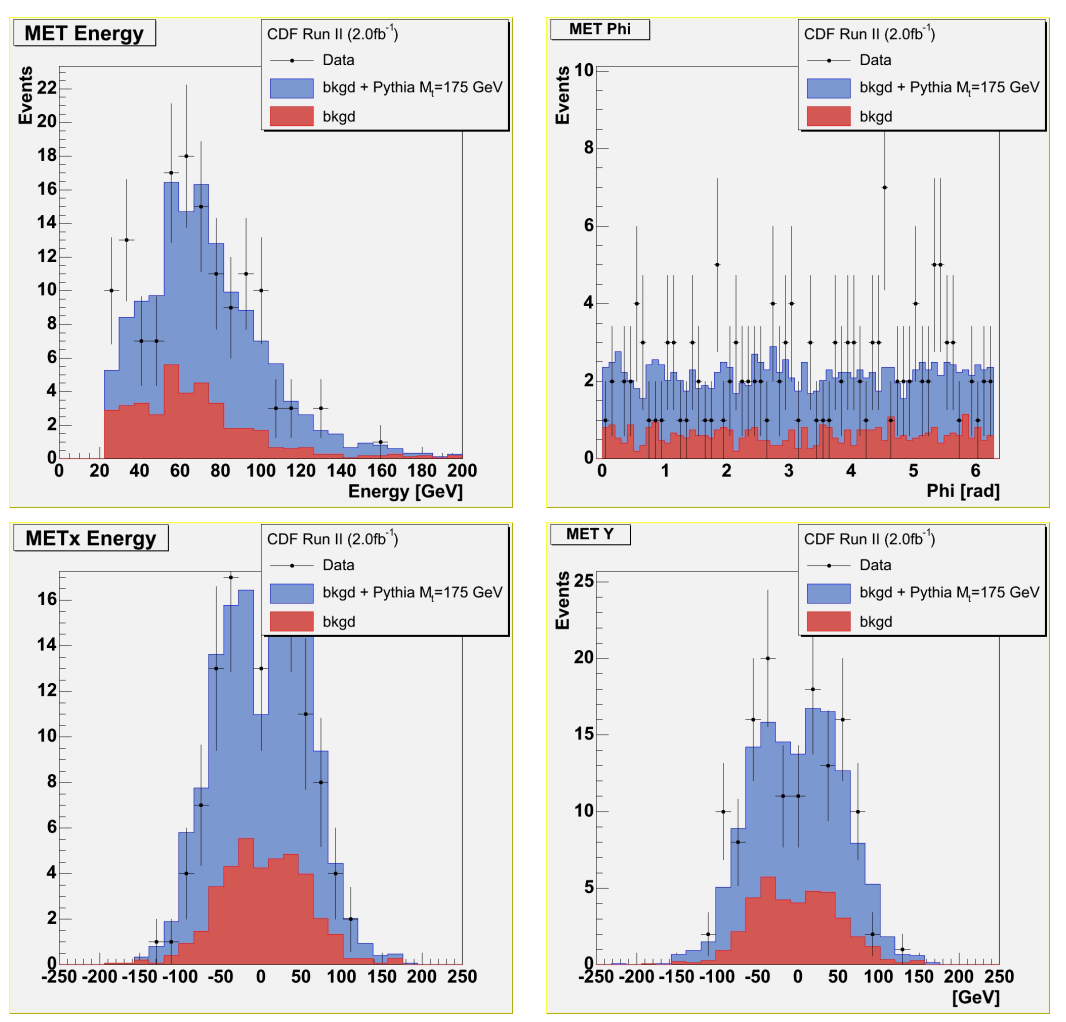

Figure 42: The energy, $\phi$, energy in the x-direction, and energy in the y-direction for the $\mathbb{E}_{T}$ in both the MC data and the real data. 


\subsection{Blind Samples}

The final check to make sure the method works is to give it ten MC data samples, each generated with a specific mass which is unknown ahead of time. Additionally, these samples do not contain a truth table. These "blind" samples can be treated just like real data with one exception. They are comprised of only $t \bar{t}$ events. The background samples have to be mixed in with the blind samples when making PEs. The results are then sent to the CDF Top Mass Group. Figure 43 shows how well the method performed with these samples. The delta (bias) plot is consistent with zero which shows that the method was able calculate the masses of the blind samples. The pull width plot has an average that is slightly less than one, which means that the errors on the mean of the mass have been over estimated. This is most likely due to the RMS correction factor, discussed in Section 5.6.1 and seen in Figures 15 and 16, being slightly too large.
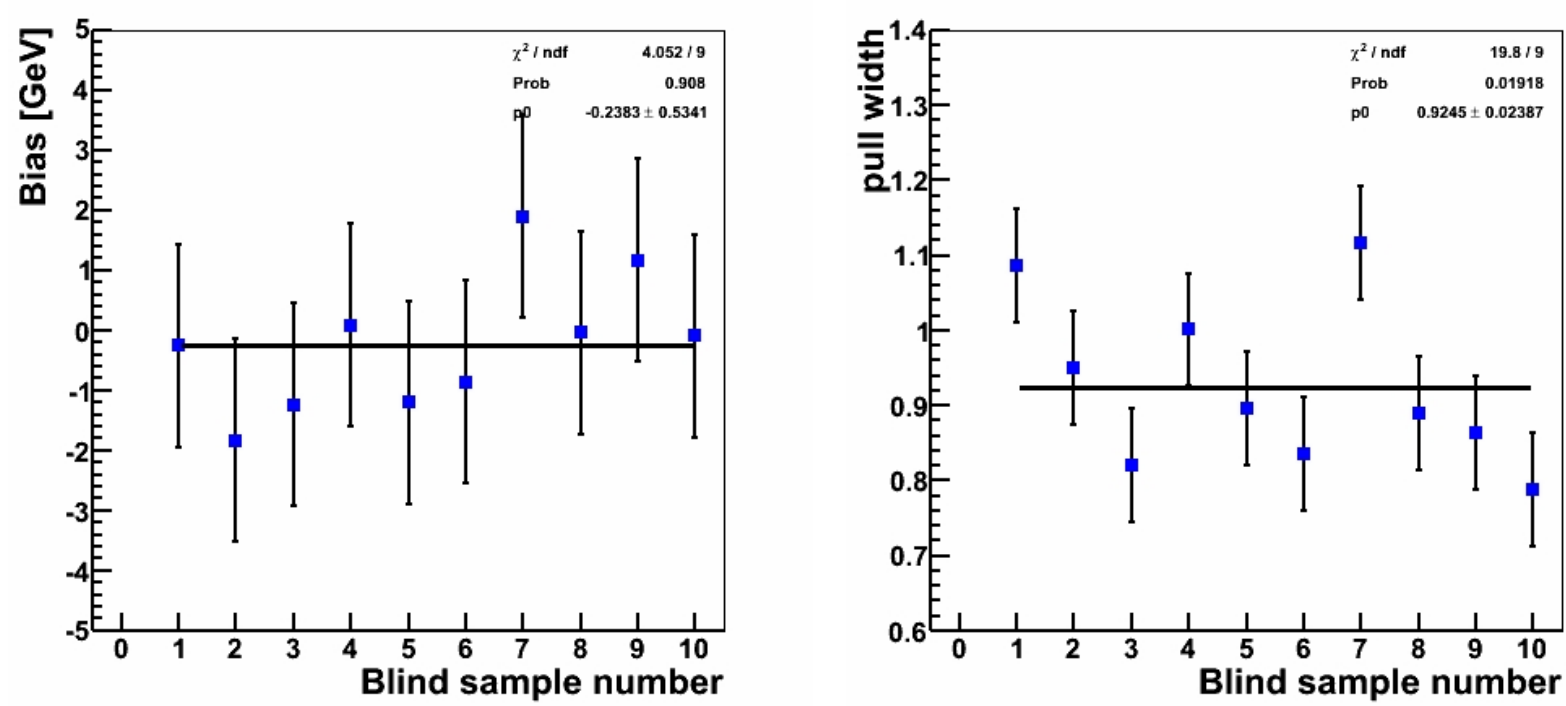

Figure 43: The delta (bias) and pull width from each of the 10 blind samples. 


\section{Chapter 6 - Systematic Errors}

\subsection{Introduction}

This chapter gives a description of the various sources of systematic errors that affect this analysis and their corresponding values. The procedures for calculating the uncertainties in this analysis were laid out by the CDF Top Group.

\subsection{Jet Energy Scale}

The majority of systematic errors in this analysis are due to the jet energy correction. As mentioned in Chapter 3, the jet energies need to be corrected for both detector and physics

effects. There are eight levels of correction, of which level 2, level 3, and level 8 are obsolete, and only three of the remaining five are used in this analysis. To calculate the systematic uncertainty, all non-obsolete levels need to be considered. Those levels are (1) relative energy correction, (4) multiple interactions, (5) absolute energy correction, (6) underlying event subtraction, and (7) out-of-cone correction. In order to calculate the uncertainty, each level of correction needs to be applied to the MC data individually. First the jet energies are shifted up by one sigma of the given correction level. From this, a mass value is calculated. Then the jet energies are all shifted down by one sigma of the given level and a mass is again calculated. These values are compared to the nominal mass calculated from applying all the levels used in this analysis normally. If the nominal value is between the two shifted values, then half of the difference between the shifted values will be used as the systematic uncertainty for the given correction level. If the nominal value is outside of the two shifted values, half of the greater difference from nominal is used as the systematic uncertainty. This process is repeated for all of the nonobsolete levels. If the statistical error for a level is greater than the shift from nominal, then the statistical error will be used as the systematic uncertainty for that level. The uncertainties for each of the levels are added in quadrature. This value is what will be used as the systematic uncertainty from the jet energy scale correction. 


\begin{tabular}{l|rr|rr|r}
\hline Correction & Mass Mean & Stat. & Mass Mean & Stat. & Syst. \\
\hline \hline All at Once & 176.57 & \pm 0.37 & 173.55 & \pm 0.39 & 1.51 \\
\hline Level 1 & 174.81 & \pm 0.55 & 175.89 & \pm 0.56 & -0.54 \\
Level 4 & 175.13 & \pm 0.55 & 175.57 & \pm 0.56 & -0.22 \\
Level 5 & 175.38 & \pm 0.55 & 175.41 & \pm 0.55 & -0.01 \\
Level 6 & 175.58 & \pm 0.55 & 174.01 & \pm 0.56 & 0.79 \\
Level 7 & 175.36 & \pm 0.52 & 174.22 & \pm 0.58 & 0.57 \\
Level 8 & 174.92 & \pm 0.55 & 175.98 & \pm 0.56 & -0.53 \\
\hline Quad Sum & \multicolumn{7}{|c}{ Shifted Up } & Shifted Down & 1.25 \\
\hline Nominal & \multicolumn{7}{|c}{$174.75 \pm 0.38$} \\
\hline
\end{tabular}

Table 16: Likelihood only mass distribution means, uncertainty on the means, and systematic uncertainties from each of the jet energy correction levels. Additionally, the sum in quadrature for all the individual levels.

\begin{tabular}{l|cr|cr|r}
\hline Correction & Mass Mean & Stat. & Mass Mean & Stat. & Syst. \\
\hline \hline All at Once & 176.60 & \pm 0.34 & 173.41 & \pm 0.36 & 1.60 \\
\hline Level 1 & 175.23 & \pm 0.52 & 176.01 & \pm 0.53 & -0.39 \\
Level 4 & 175.29 & \pm 0.52 & 175.85 & \pm 0.53 & -0.28 \\
Level 5 & 175.48 & \pm 0.52 & 175.56 & \pm 0.53 & -0.04 \\
Level 6 & 175.92 & \pm 0.52 & 173.85 & \pm 0.53 & 1.04 \\
Level 7 & 175.63 & \pm 0.50 & 174.21 & \pm 0.54 & 0.71 \\
Level 8 & 175.25 & \pm 0.52 & 176.15 & \pm 0.53 & -0.45 \\
\hline Quad Sum & Shifted Up & Shifted Down & 1.42 \\
\hline Nominal & \multicolumn{7}{|c}{$174.71 \pm 0.33$} \\
\hline
\end{tabular}

Table 17: Likelihood added mass distribution means, uncertainty on the means, and systematic uncertainties from each of the jet energy correction levels. Additionally, the sum in quadrature for all the individual levels. 


\begin{tabular}{l|rr|rr|r}
\hline Correction & Mass Mean & Stat. & Mass Mean & Stat. & Syst. \\
\hline \hline All at Once & 176.23 & \pm 0.37 & 173.09 & \pm 0.39 & 1.57 \\
\hline Level 1 & 174.70 & \pm 0.55 & 175.78 & \pm 0.57 & -0.54 \\
Level 4 & 175.10 & \pm 0.55 & 175.65 & \pm 0.56 & -0.28 \\
Level 5 & 175.34 & \pm 0.56 & 175.41 & \pm 0.56 & -0.04 \\
Level 6 & 175.66 & \pm 0.55 & 174.79 & \pm 0.56 & 0.44 \\
Level 7 & 175.49 & \pm 0.53 & 174.34 & \pm 0.58 & 0.57 \\
Level 8 & 174.81 & \pm 0.55 & 175.93 & \pm 0.56 & -0.56 \\
\hline Quad Sum & Shifted Up & Shifted Down & 1.10 \\
\hline Nominal & \multicolumn{5}{|c}{$174.44 \pm 0.38$} \\
\hline
\end{tabular}

Table 18: 2 leading jets mass distribution means, uncertainty on the means, and systematic uncertainties from each of the jet energy correction levels. Additionally, the sum in quadrature for all the individual levels.

\begin{tabular}{l|rr|cr|r}
\hline Correction & Mass Mean & Stat. & Mass Mean & Stat. & Syst. \\
\hline \hline All at Once & 176.05 & \pm 0.34 & 173.16 & \pm 0.36 & 1.45 \\
\hline Level 1 & 174.48 & \pm 0.52 & 175.42 & \pm 0.53 & -0.47 \\
Level 4 & 174.62 & \pm 0.52 & 175.41 & \pm 0.53 & -0.39 \\
Level 5 & 174.83 & \pm 0.52 & 174.97 & \pm 0.53 & -0.07 \\
Level 6 & 174.92 & \pm 0.52 & 174.03 & \pm 0.53 & 0.45 \\
Level 7 & 174.91 & \pm 0.50 & 173.88 & \pm 0.55 & 0.52 \\
Level 8 & 174.43 & \pm 0.52 & 175.60 & \pm 0.53 & -0.59 \\
\hline Quad Sum & Shifted Up & Shifted Down & 1.09 \\
\hline Nominal & \multicolumn{5}{|c}{$174.93 \pm 0.33$} \\
\hline
\end{tabular}

Table 19: 2 leading jets added mass distribution means, uncertainty on the means, and systematic uncertainties from each of the jet energy correction levels. Additionally, the sum in quadrature for all the individual levels. 
The systematic errors due to the Jet Energy Scale are:

$$
\begin{aligned}
\sigma_{J E S}^{\text {sys }}(L) & = \pm 1.25 \mathrm{GeV} & \sigma_{J E S}^{\text {sys }}(L+) & = \pm 1.42 \mathrm{GeV} \\
\sigma_{J E S}^{\text {sys }}(2 L J) & = \pm 1.10 \mathrm{GeV} & \sigma_{J E S}^{\text {sys }}(2 L J+) & = \pm 1.09 \mathrm{GeV}
\end{aligned}
$$

\subsection{PDF}

The parton distribution functions used for the MC data are the CTEQ5L set. There are 45 other PDF sets that could have been used to generate the $t \bar{t}$ events in the MC data. MRST72 is the MRST set with the same $\alpha_{s}$ as the CTEQ5L set. MRST75 is the MRST set with a different $\alpha_{s}$ as the CTEQ5L set. CTEQ6L and CTEQ6L1 are two CTEQ sets with different $\alpha_{s}$ values, one loop and two loop respectively. CTEQ6M $N \pm$, where $N$ has values from 1 to 20, are 20 pairs of orthogonal eigenvector PDF sets and CTEQ6M is the set to which they will need to be compared. Instead of generating 45 additional data sets, the $175 \mathrm{GeV} \mathrm{MC}$ data set from the CTEQ5L pdf is re-weighted. For every event, the truth table is searched to find which part of the proton, i.e. an up quark, hit which part of the anti-proton, i.e. a gluon, in addition to the $q^{2}$ of the interaction. Given each of the parton's momentum fractions, $x 1$ and $x 2$, a probability that they will interact with a momentum transfer of $q^{2}$ is calculated for each PDF. The probability for a given event and PDF is used as a weighting factor in the analysis. The mass distribution for a given event is multiplied by this factor before it is used in any pseudo-experiments, which effectively alters its relative probability compared to other events in a sample. This is done for each of the 46 PDF sets, and a mass value is calculated for each set. Table 3 shows the mass value and uncertainty for each of the PDF sets.

To calculate the systematic uncertainty from the different PDFs, first the differences from the CTEQ6MN土 set and the CTEQ6M set are calculated. These are added in quadrature asymmetrically. This is referred to the eigenvector uncertainty. Next, the difference between the MRST72 and CTEQ5L sets is calculated. This is the MRST uncertainty. Third, the difference between the MRST72 and MRST75 sets is calculated. This is the $\alpha_{s}$ uncertainty. Table 4 shows all of these individual uncertainties. 


\begin{tabular}{|lrr||lrr|}
\hline PDF Set & Mass Mean & Uncertainty & PDF Set & Mass Mean & Uncertainty \\
\hline \hline CTEQ5L & 173.76 & \pm 0.67 & MRST72 & 173.66 & \pm 0.66 \\
MRST75 & 174.00 & \pm 0.64 & CTEQ6L & 173.76 & \pm 0.67 \\
CTEQ6L1 & 173.65 & \pm 0.68 & CTEQ6M & 173.78 & \pm 0.66 \\
CTEQ6M1+ & 173.83 & \pm 0.65 & CTEQ6M1- & 173.73 & \pm 0.66 \\
CTEQ6M2+ & 173.68 & \pm 0.66 & CTEQ6M2- & 173.66 & \pm 0.65 \\
CTEQ6M3+ & 173.68 & \pm 0.67 & CTEQ6M3- & 173.79 & \pm 0.66 \\
CTEQ6M4+ & 173.68 & \pm 0.65 & CTEQ6M4- & 173.66 & \pm 0.66 \\
CTEQ6M5+ & 173.62 & \pm 0.66 & CTEQ6M5- & 173.74 & \pm 0.66 \\
CTEQ6M6+ & 173.76 & \pm 0.66 & CTEQ6M6- & 173.77 & \pm 0.66 \\
CTEQ6M7+ & 173.69 & \pm 0.66 & CTEQ6M7- & 173.64 & \pm 0.65 \\
CTEQ6M8+ & 173.70 & \pm 0.67 & CTEQ6M8- & 173.79 & \pm 0.66 \\
CTEQ6M9+ & 173.70 & \pm 0.65 & CTEQ6M9- & 173.66 & \pm 0.66 \\
CTEQ6M10+ & 173.68 & \pm 0.66 & CTEQ6M10- & 173.79 & \pm 0.66 \\
CTEQ6M11+ & 173.76 & \pm 0.66 & CTEQ6M11- & 173.78 & \pm 0.66 \\
CTEQ6M12+ & 173.66 & \pm 0.66 & CTEQ6M12- & 173.62 & \pm 0.66 \\
CTEQ6M13+ & 173.67 & \pm 0.67 & CTEQ6M13- & 173.79 & \pm 0.66 \\
CTEQ6M14+ & 173.72 & \pm 0.65 & CTEQ6M14- & 173.64 & \pm 0.66 \\
CTEQ6M15+ & 173.65 & \pm 0.67 & CTEQ6M15- & 173.76 & \pm 0.59 \\
CTEQ6M16+ & 173.76 & \pm 0.66 & CTEQ6M16- & 173.72 & \pm 0.66 \\
CTEQ6M17+ & 173.66 & \pm 0.66 & CTEQ6M17- & 173.62 & \pm 0.65 \\
CTEQ6M18+ & 173.69 & \pm 0.67 & CTEQ6M18- & 173.79 & \pm 0.65 \\
CTEQ6M19+ & 173.72 & \pm 0.64 & CTEQ6M19- & 173.63 & \pm 0.66 \\
CTEQ6M20+ & 173.65 & \pm 0.66 & CTEQ6M20- & 173.77 & \pm 0.66 \\
\hline
\end{tabular}

Table 20: Likelihood only mass distribution means and uncertainty on the means from each of the 46 PDFs 


\begin{tabular}{|lrr||lrr|}
\hline PDF Set & Mass Mean & Uncertainty & PDF Set & Mass Mean & Uncertainty \\
\hline \hline CTEQ5L & 173.59 & \pm 0.56 & MRST72 & 173.52 & \pm 0.56 \\
MRST75 & 173.82 & \pm 0.55 & CTEQ6L & 173.59 & \pm 0.56 \\
CTEQ6L1 & 173.53 & \pm 0.57 & CTEQ6M & 173.75 & \pm 0.57 \\
CTEQ6M1+ & 173.78 & \pm 0.56 & CTEQ6M1- & 173.76 & \pm 0.57 \\
CTEQ6M2+ & 173.63 & \pm 0.56 & CTEQ6M2- & 173.57 & \pm 0.56 \\
CTEQ6M3+ & 173.60 & \pm 0.56 & CTEQ6M3- & 173.67 & \pm 0.56 \\
CTEQ6M4+ & 173.67 & \pm 0.56 & CTEQ6M4- & 173.61 & \pm 0.55 \\
CTEQ6M5+ & 173.57 & \pm 0.56 & CTEQ6M5- & 173.69 & \pm 0.57 \\
CTEQ6M6+ & 173.70 & \pm 0.56 & CTEQ6M6- & 173.81 & \pm 0.57 \\
CTEQ6M7+ & 173.64 & \pm 0.56 & CTEQ6M7- & 173.55 & \pm 0.56 \\
CTEQ6M8+ & 173.62 & \pm 0.56 & CTEQ6M8- & 173.69 & \pm 0.56 \\
CTEQ6M9+ & 173.67 & \pm 0.56 & CTEQ6M9- & 173.61 & \pm 0.55 \\
CTEQ6M10+ & 173.56 & \pm 0.56 & CTEQ6M10- & 173.79 & \pm 0.57 \\
CTEQ6M11+ & 173.72 & \pm 0.56 & CTEQ6M11- & 173.81 & \pm 0.56 \\
CTEQ6M12+ & 173.61 & \pm 0.56 & CTEQ6M12- & 173.56 & \pm 0.57 \\
CTEQ6M13+ & 173.58 & \pm 0.56 & CTEQ6M13- & 173.71 & \pm 0.56 \\
CTEQ6M14+ & 173.67 & \pm 0.55 & CTEQ6M14- & 173.60 & \pm 0.56 \\
CTEQ6M15+ & 173.52 & \pm 0.56 & CTEQ6M15- & 173.79 & \pm 0.52 \\
CTEQ6M16+ & 173.73 & \pm 0.56 & CTEQ6M16- & 173.75 & \pm 0.57 \\
CTEQ6M17+ & 173.61 & \pm 0.57 & CTEQ6M17- & 173.53 & \pm 0.56 \\
CTEQ6M18+ & 173.61 & \pm 0.56 & CTEQ6M18- & 173.70 & \pm 0.56 \\
CTEQ6M19+ & 173.76 & \pm 0.55 & CTEQ6M19- & 173.59 & \pm 0.56 \\
CTEQ6M20+ & 173.58 & \pm 0.56 & CTEQ6M20- & 173.73 & \pm 0.57 \\
\hline
\end{tabular}

Table 21: Likelihood added mass distribution means and uncertainty on the means from each of the 46 PDFs 


\begin{tabular}{|lrr||lrr|}
\hline PDF Set & Mass Mean & Uncertainty & PDF Set & Mass Mean & Uncertainty \\
\hline \hline CTEQ5L & 175.65 & \pm 0.83 & MRST72 & 175.58 & \pm 0.82 \\
MRST75 & 175.78 & \pm 0.78 & CTEQ6L & 175.65 & \pm 0.84 \\
CTEQ6L1 & 175.64 & \pm 0.86 & CTEQ6M & 175.69 & \pm 0.82 \\
CTEQ6M1+ & 175.75 & \pm 0.80 & CTEQ6M1- & 175.64 & \pm 0.82 \\
CTEQ6M2+ & 175.69 & \pm 0.83 & CTEQ6M2- & 175.74 & \pm 0.81 \\
CTEQ6M3+ & 175.60 & \pm 0.80 & CTEQ6M3- & 175.68 & \pm 0.81 \\
CTEQ6M4+ & 175.63 & \pm 0.81 & CTEQ6M4- & 175.63 & \pm 0.81 \\
CTEQ6M5+ & 175.72 & \pm 0.82 & CTEQ6M5- & 175.67 & \pm 0.83 \\
CTEQ6M6+ & 175.66 & \pm 0.81 & CTEQ6M6- & 175.70 & \pm 0.82 \\
CTEQ6M7+ & 175.68 & \pm 0.83 & CTEQ6M7- & 175.74 & \pm 0.81 \\
CTEQ6M8+ & 175.63 & \pm 0.80 & CTEQ6M8- & 175.65 & \pm 0.81 \\
CTEQ6M9+ & 175.63 & \pm 0.81 & CTEQ6M9- & 175.63 & \pm 0.81 \\
CTEQ6M10+ & 175.66 & \pm 0.82 & CTEQ6M10- & 175.72 & \pm 0.82 \\
CTEQ6M11+ & 175.68 & \pm 0.81 & CTEQ6M11- & 175.68 & \pm 0.82 \\
CTEQ6M12+ & 175.68 & \pm 0.84 & CTEQ6M12- & 175.72 & \pm 0.82 \\
CTEQ6M13+ & 175.58 & \pm 0.80 & CTEQ6M13- & 175.66 & \pm 0.81 \\
CTEQ6M14+ & 175.64 & \pm 0.81 & CTEQ6M14- & 175.62 & \pm 0.81 \\
CTEQ6M15+ & 175.59 & \pm 0.84 & CTEQ6M15- & 175.70 & \pm 0.76 \\
CTEQ6M16+ & 175.70 & \pm 0.81 & CTEQ6M16- & 175.63 & \pm 0.82 \\
CTEQ6M17+ & 175.67 & \pm 0.84 & CTEQ6M17- & 175.76 & \pm 0.82 \\
CTEQ6M18+ & 175.60 & \pm 0.80 & CTEQ6M18- & 175.65 & \pm 0.81 \\
CTEQ6M19+ & 175.64 & \pm 0.79 & CTEQ6M19- & 175.58 & \pm 0.81 \\
CTEQ6M20+ & 175.69 & \pm 0.82 & CTEQ6M20- & 175.69 & \pm 0.83 \\
\hline
\end{tabular}

Table 22: 2 leading jets mass distribution means and uncertainty on the means from each of the 46 PDFs 


\begin{tabular}{|lrr||lrr|}
\hline PDF Set & Mass Mean & Uncertainty & PDF Set & Mass Mean & Uncertainty \\
\hline \hline CTEQ5L & 173.26 & \pm 0.60 & MRST72 & 173.18 & \pm 0.60 \\
MRST75 & 173.36 & \pm 0.58 & CTEQ6L & 173.24 & \pm 0.60 \\
CTEQ6L1 & 173.20 & \pm 0.61 & CTEQ6M & 173.27 & \pm 0.60 \\
CTEQ6M1+ & 173.32 & \pm 0.61 & CTEQ6M1- & 173.22 & \pm 0.61 \\
CTEQ6M2+ & 173.22 & \pm 0.59 & CTEQ6M2- & 173.29 & \pm 0.60 \\
CTEQ6M3+ & 173.25 & \pm 0.59 & CTEQ6M3- & 173.31 & \pm 0.60 \\
CTEQ6M4+ & 173.19 & \pm 0.60 & CTEQ6M4- & 173.29 & \pm 0.59 \\
CTEQ6M5+ & 173.23 & \pm 0.60 & CTEQ6M5- & 173.26 & \pm 0.60 \\
CTEQ6M6+ & 173.24 & \pm 0.61 & CTEQ6M6- & 173.29 & \pm 0.61 \\
CTEQ6M7+ & 173.23 & \pm 0.59 & CTEQ6M7- & 173.28 & \pm 0.60 \\
CTEQ6M8+ & 173.28 & \pm 0.60 & CTEQ6M8- & 173.29 & \pm 0.60 \\
CTEQ6M9+ & 173.19 & \pm 0.60 & CTEQ6M9- & 173.30 & \pm 0.60 \\
CTEQ6M10+ & 173.20 & \pm 0.60 & CTEQ6M10- & 173.29 & \pm 0.60 \\
CTEQ6M11+ & 173.26 & \pm 0.61 & CTEQ6M11- & 173.27 & \pm 0.60 \\
CTEQ6M12+ & 173.19 & \pm 0.59 & CTEQ6M12- & 173.27 & \pm 0.60 \\
CTEQ6M13+ & 173.24 & \pm 0.60 & CTEQ6M13- & 173.30 & \pm 0.60 \\
CTEQ6M14+ & 173.22 & \pm 0.60 & CTEQ6M14- & 173.28 & \pm 0.60 \\
CTEQ6M15+ & 173.16 & \pm 0.60 & CTEQ6M15- & 173.36 & \pm 0.58 \\
CTEQ6M16+ & 173.26 & \pm 0.61 & CTEQ6M16- & 173.21 & \pm 0.61 \\
CTEQ6M17+ & 173.19 & \pm 0.59 & CTEQ6M17- & 173.29 & \pm 0.59 \\
CTEQ6M18+ & 173.25 & \pm 0.59 & CTEQ6M18- & 173.30 & \pm 0.60 \\
CTEQ6M19+ & 173.23 & \pm 0.59 & CTEQ6M19- & 173.26 & \pm 0.60 \\
CTEQ6M20+ & 173.21 & \pm 0.60 & CTEQ6M20- & 173.27 & \pm 0.60 \\
\hline
\end{tabular}

Table 23: 2 leading jets mass distribution means and uncertainty on the means from each of the 46 PDFs 


\begin{tabular}{|lcr||lrr|}
\hline Uncert. & PDF Sets & Diff. & Uncert. & \multicolumn{1}{c}{ PDF Sets } & Diff. \\
\hline \hline MRST & MRST72 - CTEQ5L & -0.10 & $\alpha_{s}$ & MRST75 - MRST72 & 0.34 \\
\hline EV1+ & CTEQ6M1+ - CTEQ6M & 0.06 & EV1- & CTEQ6M1- - CTEQ6M & -0.05 \\
EV2+ & CTEQ6M2+ - CTEQ6M & -0.09 & EV2- & CTEQ6M2- - CTEQ6M & -0.12 \\
EV3+ & CTEQ6M3+ - CTEQ6M & -0.10 & EV3- & CTEQ6M3- - CTEQ6M & 0.02 \\
EV4+ & CTEQ6M4+ - CTEQ6M & -0.09 & EV4- & CTEQ6M4- - CTEQ6M & -0.12 \\
EV5+ & CTEQ6M5+ - CTEQ6M & -0.15 & EV5- & CTEQ6M5- - CTEQ6M & -0.04 \\
EV6+ & CTEQ6M6+ - CTEQ6M & -0.02 & EV6- & CTEQ6M6- - CTEQ6M & -0.00 \\
EV7+ & CTEQ6M7+ - CTEQ6M & -0.08 & EV7- & CTEQ6M7- - CTEQ6M & -0.14 \\
EV8+ & CTEQ6M8+ - CTEQ6M & -0.08 & EV8- & CTEQ6M8- - CTEQ6M & 0.02 \\
EV9+ & CTEQ6M9+ - CTEQ6M & -0.07 & EV9- & CTEQ6M9- - CTEQ6M & -0.11 \\
EV10+ & CTEQ6M10+ - CTEQ6M & -0.10 & EV10- & CTEQ6M10- - CTEQ6M & 0.02 \\
EV11+ & CTEQ6M11+ - CTEQ6M & -0.01 & EV11- & CTEQ6M11- CTEQ6M & 0.00 \\
EV12+ & CTEQ6M12+ - CTEQ6M & -0.11 & EV12- & CTEQ6M12- - CTEQ6M & -0.15 \\
EV13+ & CTEQ6M13+ - CTEQ6M & -0.10 & EV13- & CTEQ6M13- CTEQ6M & 0.02 \\
EV14+ & CTEQ6M14+ - CTEQ6M & -0.06 & EV14- & CTEQ6M14- - CTEQ6M & -0.14 \\
EV15+ & CTEQ6M15+ - CTEQ6M & -0.13 & EV15- & CTEQ6M15- CTEQ6M & -0.02 \\
EV16+ & CTEQ6M16+ - CTEQ6M & -0.01 & EV16- & CTEQ6M16- - CTEQ6M & -0.05 \\
EV17+ & CTEQ6M17+ - CTEQ6M & -0.11 & EV17- & CTEQ6M17- CTEQ6M & -0.15 \\
EV18+ & CTEQ6M18+ - CTEQ6M & -0.09 & EV18- & CTEQ6M18- CTEQ6M & 0.01 \\
EV19+ & CTEQ6M19+ - CTEQ6M & -0.06 & EV19- & CTEQ6M19- CTEQ6M & -0.15 \\
EV20+ & CTEQ6M20+ - CTEQ6M & -0.12 & EV20- & CTEQ6M20- - CTEQ6M & -0.00 \\
\hline EV+ STm & in Quadrature & 0.07 & EV- Sum & in Quadrature & 0.50 \\
\hline
\end{tabular}

Table 24: Likelihood only uncertainties for the different PDFs 


\begin{tabular}{|c|c|c|c|c|c|}
\hline Uncert. & PDF Sets & Diff. & Uncert. & PDF Sets & Diff. \\
\hline MRST & MRST72 - CTEQ5L & -0.07 & $\overline{\alpha \alpha_{s}}$ & MRST75 - MRST72 & 0.30 \\
\hline EV1+ & CTEQ6M1+ - CTEQ6M & 0.04 & EV1- & CTEQ6M1- - CTEQ6M & 0.01 \\
\hline $\mathrm{EV} 2+$ & CTEQ6M2+ - CTEQ6M & -0.12 & EV2- & CTEQ6M2- - CTEQ6M & -0.18 \\
\hline EV3+ & CTEQ6M3+ - CTEQ6M & -0.15 & EV3- & CTEQ6M3- - CTEQ6M & -0.08 \\
\hline EV4+ & CTEQ6M4+ - CTEQ6M & -0.08 & EV4- & CTEQ6M4- - CTEQ6M & -0.14 \\
\hline EV5+ & CTEQ6M5+ - CTEQ6M & -0.18 & EV5- & CTEQ6M5- - CTEQ6M & -0.06 \\
\hline EV6+ & CTEQ6M6+ - CTEQ6M & -0.05 & EV6- & CTEQ6M6- - CTEQ6M & 0.07 \\
\hline EV7+ & CTEQ6M7+ - CTEQ6M & -0.11 & EV7- & CTEQ6M7- - CTEQ6M & -0.20 \\
\hline EV8+ & CTEQ6M8+ - CTEQ6M & -0.13 & EV8- & CTEQ6M8- - CTEQ6M & -0.06 \\
\hline EV9+ & CTEQ6M9+ - CTEQ6M & -0.07 & EV9- & CTEQ6M9- - CTEQ6M & -0.14 \\
\hline EV10+ & CTEQ6M10+ - CTEQ6M & -0.18 & EV10- & CTEQ6M10- - CTEQ6M & 0.04 \\
\hline EV11+ & CTEQ6M11+ - CTEQ6M & -0.03 & EV11- & CTEQ6M11- - CTEQ6M & 0.06 \\
\hline EV12+ & CTEQ6M12+ - CTEQ6M & -0.14 & EV12- & CTEQ6M12- - CTEQ6M & -0.19 \\
\hline EV13+ & CTEQ6M13+ - CTEQ6M & -0.16 & EV13- & CTEQ6M13- - CTEQ6M & -0.04 \\
\hline EV14+ & CTEQ6M14+ - CTEQ6M & -0.08 & EV14- & CTEQ6M14- - CTEQ6M & -0.14 \\
\hline EV15+ & CTEQ6M15+ - CTEQ6M & -0.22 & EV15- & CTEQ6M15- - CTEQ6M & 0.04 \\
\hline EV16+ & CTEQ6M16+ - CTEQ6M & -0.01 & EV16- & CTEQ6M16- - CTEQ6M & 0.00 \\
\hline EV17+ & CTEQ6M17+ - CTEQ6M & -0.14 & EV17- & CTEQ6M17- - CTEQ6M & -0.22 \\
\hline EV18+ & CTEQ6M18+ - CTEQ6M & -0.14 & EV18- & CTEQ6M18- - CTEQ6M & -0.05 \\
\hline EV19+ & CTEQ6M19+ - CTEQ6M & 0.01 & EV19- & CTEQ6M19- - CTEQ6M & -0.16 \\
\hline $\mathrm{EV} 20+$ & CTEQ6M20+ - CTEQ6M & -0.17 & EV20- & CTEQ6M20- - CTEQ6M & -0.01 \\
\hline \multicolumn{2}{|c|}{$\mathrm{EV}+$ Sum in Quadrature } & 0.12 & \multicolumn{2}{|c|}{ EV- Sum in Quadrature } & 0.68 \\
\hline
\end{tabular}

Table 25: Likelihood added uncertainties for the different PDFs 


\begin{tabular}{|lcr||lrr|}
\hline Uncert. & PDF Sets & Diff. & Uncert. & \multicolumn{1}{c}{ PDF Sets } & Diff. \\
\hline \hline MRST & MRST72 - CTEQ5L & -0.07 & $\alpha_{s}$ & MRST75 - MRST72 & 0.20 \\
\hline EV1+ & CTEQ6M1+ - CTEQ6M & 0.06 & EV1- & CTEQ6M1- - CTEQ6M & -0.06 \\
EV2+ & CTEQ6M2+ - CTEQ6M & 0.00 & EV2- & CTEQ6M2- - CTEQ6M & 0.05 \\
EV3+ & CTEQ6M3+ - CTEQ6M & -0.09 & EV3- & CTEQ6M3- - CTEQ6M & -0.01 \\
EV4+ & CTEQ6M4+ - CTEQ6M & -0.06 & EV4- & CTEQ6M4- - CTEQ6M & -0.06 \\
EV5+ & CTEQ6M5+ - CTEQ6M & 0.03 & EV5- & CTEQ6M5- - CTEQ6M & -0.02 \\
EV6+ & CTEQ6M6+ - CTEQ6M & -0.03 & EV6- & CTEQ6M6- - CTEQ6M & 0.01 \\
EV7+ & CTEQ6M7+ - CTEQ6M & -0.01 & EV7- & CTEQ6M7- - CTEQ6M & 0.05 \\
EV8+ & CTEQ6M8+ - CTEQ6M & -0.06 & EV8- & CTEQ6M8- - CTEQ6M & -0.04 \\
EV9+ & CTEQ6M9+ - CTEQ6M & -0.06 & EV9- & CTEQ6M9- - CTEQ6M & -0.06 \\
EV10+ & CTEQ6M10+ - CTEQ6M & -0.03 & EV10- & CTEQ6M10- - CTEQ6M & 0.03 \\
EV11+ & CTEQ6M11+ - CTEQ6M & -0.01 & EV11- & CTEQ6M11- CTEQ6M & -0.01 \\
EV12+ & CTEQ6M12+ - CTEQ6M & -0.02 & EV12- & CTEQ6M12- - CTEQ6M & 0.03 \\
EV13+ & CTEQ6M13+ - CTEQ6M & -0.11 & EV13- & CTEQ6M13- CTEQ6M & -0.03 \\
EV14+ & CTEQ6M14+ - CTEQ6M & -0.05 & EV14- & CTEQ6M14- - CTEQ6M & -0.07 \\
EV15+ & CTEQ6M15+ - CTEQ6M & -0.10 & EV15- & CTEQ6M15- CTEQ6M & 0.01 \\
EV16+ & CTEQ6M16+ - CTEQ6M & 0.00 & EV16- & CTEQ6M16- - CTEQ6M & -0.06 \\
EV17+ & CTEQ6M17+ - CTEQ6M & -0.03 & EV17- & CTEQ6M17- CTEQ6M & 0.07 \\
EV18+ & CTEQ6M18+ - CTEQ6M & -0.09 & EV18- & CTEQ6M18- CTEQ6M & -0.04 \\
EV19+ & CTEQ6M19+ - CTEQ6M & -0.05 & EV19- & CTEQ6M19- CTEQ6M & -0.11 \\
EV20+ & CTEQ6M20+ - CTEQ6M & -0.00 & EV20- & CTEQ6M20- - CTEQ6M & 0.00 \\
\hline EV+ STm & in Quadrature & 0.13 & EV- Sum & in Quadrature & 0.28 \\
\hline
\end{tabular}

Table 26: 2 leading jets uncertainties for the different PDFs 


\begin{tabular}{|c|c|c|c|c|c|}
\hline Uncert. & PDF Sets & Diff. & Uncert. & PDF Sets & Diff. \\
\hline MRST & IMRST72 - CTEQ5L & -0.08 & $\alpha_{s}$ & "MRST75 - MRST72 & 0.17 \\
\hline EV1+ & CTEQ6M1+ - CTEQ6M & 0.05 & EV1- & CTEQ6M1- - CTEQ6M & -0.05 \\
\hline $\mathrm{EV} 2+$ & CTEQ6M2+ - CTEQ6M & -0.05 & EV2- & CTEQ6M2- - CTEQ6M & 0.02 \\
\hline $\mathrm{EV} 3+$ & CTEQ6M3+ - CTEQ6M & -0.02 & EV3- & CTEQ6M3- - CTEQ6M & 0.04 \\
\hline EV4+ & CTEQ6M4+ - CTEQ6M & -0.08 & EV4- & CTEQ6M4- - CTEQ6M & 0.02 \\
\hline EV5+ & CTEQ6M5 + - CTEQ6M & -0.03 & EV5- & CTEQ6M5- - CTEQ6M & -0.01 \\
\hline $\mathrm{EV} 6+$ & CTEQ6M6+ - CTEQ6M & -0.03 & EV6- & CTEQ6M6- - CTEQ6M & 0.02 \\
\hline EV7+ & CTEQ6M7+ - CTEQ6M & -0.04 & EV7- & CTEQ6M7- - CTEQ6M & 0.01 \\
\hline EV8+ & CTEQ6M8+ - CTEQ6M & 0.01 & EV8- & CTEQ6M8- - CTEQ6M & 0.02 \\
\hline EV9+ & CTEQ6M9+ - CTEQ6M & -0.08 & EV9- & CTEQ6M9- - CTEQ6M & 0.03 \\
\hline EV10+ & CTEQ6M10+ - CTEQ6M & -0.07 & EV10- & CTEQ6M10- - CTEQ6M & 0.02 \\
\hline EV11+ & CTEQ6M11 + - CTEQ6M & -0.01 & EV11- & CTEQ6M11- - CTEQ6M & 0.00 \\
\hline EV12+ & CTEQ6M12+ - CTEQ6M & -0.08 & EV12- & CTEQ6M12- - CTEQ6M & -0.00 \\
\hline EV13+ & CTEQ6M13+ - CTEQ6M & -0.03 & EV13- & CTEQ6M13- - CTEQ6M & 0.03 \\
\hline EV14+ & CTEQ6M14+ - CTEQ6M & -0.05 & EV14- & CTEQ6M14- - CTEQ6M & 0.01 \\
\hline EV15+ & CTEQ6M15+ - CTEQ6M & -0.11 & EV15- & CTEQ6M15- - CTEQ6M & 0.09 \\
\hline EV16+ & CTEQ6M16+ - CTEQ6M & -0.01 & EV16- & CTEQ6M16- - CTEQ6M & -0.06 \\
\hline EV17+ & CTEQ6M17+ - CTEQ6M & -0.08 & EV17- & CTEQ6M17- - CTEQ6M & 0.02 \\
\hline EV18+ & CTEQ6M18+ - CTEQ6M & -0.02 & EV18- & CTEQ6M18- - CTEQ6M & 0.03 \\
\hline EV19+ & CTEQ6M19+ - CTEQ6M & -0.04 & EV19- & CTEQ6M19- - CTEQ6M & -0.01 \\
\hline EV20+ & CTEQ6M20+ - CTEQ6M & -0.06 & EV20- & CTEQ6M20- - CTEQ6M & -0.00 \\
\hline \multicolumn{2}{|c|}{ EV+ Sum in Quadrature } & 0.13 & \multicolumn{2}{|c|}{ EV-Sum in Quadrature } & 0.25 \\
\hline
\end{tabular}

Table 27: 2 leading jets added uncertainties for the different PDFs 
The total systematic uncertainty from the PDFs will be the greater of the MRST and eigenvector uncertainties added in quadrature to the $\alpha_{s}$ uncertainty.

The systematic error due to the PDFs are:

$$
\begin{aligned}
\sigma_{P D F}^{\text {sys }}(L) & ={ }_{-0.78}^{+0.35} \mathrm{GeV} & \sigma_{P D F}^{\text {sys }}(L+) & ={ }_{-0.74}^{+0.32} \mathrm{GeV} \\
\sigma_{P D F}^{\text {sys }}(2 L J) & ={ }_{-0.34}^{+0.24} \mathrm{GeV} & \sigma_{P D F}^{\text {sys }}(2 L J+) & ={ }_{-0.30}^{+0.21} \mathrm{GeV}
\end{aligned}
$$

\subsection{ISR/FSR}

High $E_{t}$ gluons that are radiated either before the production of a $t \bar{t}$ pair, initial state radiation (ISR), or after the production of a $t \bar{t}$ pair, final state radiation (FSR), can alter both the jet multiplicity of an event and the energy distribution among the jets. To calculate a systematic uncertainty from these effects, two MC data sets were generated. The first has the ISR and FSR turned off. The second has both turned on at twice the normal rate. In the past, this study would have been done with four MC sets: two for the ISR and two for the FSR. Currently, only two sets are used, and ISR and FSR are either turned on at twice the rate, or are turned off simultaneously in both sets. To make the calculation, both samples are compared to the nominal. The larger difference is taken as the systematic uncertainty.

\begin{tabular}{lcccc}
\hline MC Sample & Likelihood & L Added & 2LJ & 2LJ Added \\
\hline \hline ttop75 & $174.75 \pm 0.38$ & $174.71 \pm 0.33$ & $174.44 \pm 0.38$ & $174.93 \pm 0.33$ \\
otop03 & $174.81 \pm 0.33$ & $174.67 \pm 0.31$ & $175.31 \pm 0.33$ & $175.10 \pm 0.30$ \\
otop04 & $174.44 \pm 0.27$ & $175.17 \pm 0.25$ & $174.23 \pm 0.27$ & $175.03 \pm 0.25$ \\
\hline
\end{tabular}

Table 28: Mass distribution means and the uncertainty on the means for the IFS/FSR systematic samples.

\begin{tabular}{lcccc}
\hline MC Sample & Likelihood & L Added & 2LJ & 2LJ Added \\
\hline \hline otopo3 & $0.06 \pm 0.50$ & $-0.04 \pm 0.45$ & $0.87 \pm 0.51$ & $0.17 \pm 0.45$ \\
otopo4 & $-0.31 \pm 0.47$ & $0.46 \pm 0.41$ & $-0.21 \pm 0.47$ & $0.10 \pm 0.41$ \\
\hline
\end{tabular}

Table 29: Difference of the ISR/FSR systematic samples from the nominal sample. 
The systematic errors due to Initial and Final State Radiation are:

$$
\begin{aligned}
& \sigma_{I F S R}^{\text {sys }}(L)= \pm 0.38 G e V \quad \sigma_{I F S R}^{\text {sys }}(L+)= \pm 0.46 G e V \\
& \sigma_{I F S R}^{\text {sys }}(2 L J)= \pm 0.87 G e V \quad \sigma_{I F S R}^{\text {sys }}(2 L J+)= \pm 0.33 G e V
\end{aligned}
$$

\subsection{Generator}

The main MC generator for this analysis has been PYTHIA. In order to calculate a systematic uncertainty from the choice of generator, a $175 \mathrm{GeV}$ mass sample generated by HERWIG is used. The difference between the the mass values for each of these generators is the systematic uncertainty.

\begin{tabular}{lcccc}
\hline MC Sample & Likelihood & L Added & 2LJ & 2LJ Added \\
\hline \hline ttop75 & $174.75 \pm 0.38$ & $174.71 \pm 0.33$ & $174.44 \pm 0.38$ & $174.93 \pm 0.33$ \\
otop1s & $175.37 \pm 0.43$ & $174.81 \pm 0.37$ & $175.04 \pm 0.43$ & $174.70 \pm 0.37$ \\
\hline \hline Shift & $-0.62 \pm 0.57$ & $-0.10 \pm 0.50$ & $-0.61 \pm 0.57$ & $0.23 \pm 0.50$ \\
\hline
\end{tabular}

Table 30: Mass distribution means and the uncertainty on the means for the PYTHIA and HERWIG samples as well as the difference of the HERWIG samples from the nominal (PYTHIA) sample.

The systematic errors due to different MC Generators are:

$$
\begin{array}{cc}
\sigma_{M C}^{\text {sys }}(L)= \pm 0.62 G e V & \sigma_{M C}^{\text {sys }}(L+)= \pm 0.33 G e V \\
\sigma_{M C}^{\text {sys }}(2 L J) & = \pm 0.61 G e V \quad \sigma_{M C}^{\text {sys }}(2 L J+)= \pm 0.33 G e V
\end{array}
$$

\subsection{Background Fraction}

As can be seen in Chapter 5, the addition of background will alter the mapping function used to make the correction to the top mass measurement. Changes in the composition of the background sources will also have an effect and are a source of a systematic uncertainty. Table 13 
shows the number of events expected in the real data, and the uncertainty on those numbers. To calculate the systematic uncertainty from each type of background, the number of expected events for a given background is shifted up and down by one sigma. In addition to shifting each background, the total number of background events is also shifted up and down. This will result in nine pairs of mass values, as shown in Tables 6.6 through 6.6, where the difference from each pair is a systematic error from that background source. Each of the differences are added in quadrature, and the larger of either that sum or the statistical uncertainty on the nominal sample is used as the systematic error due to the background fraction.

\begin{tabular}{l|c|c|r}
\hline Background & Mass Mean & Mass Mean & Diff/2 \\
\hline \hline Fakes & 174.64 & 175.21 & -0.29 \\
$W W$ & 174.78 & 175.02 & -0.12 \\
$W Z$ & 174.85 & 174.93 & -0.04 \\
$\mathrm{ZZ}$ & 174.81 & 174.99 & -0.09 \\
$\mathrm{~W} \gamma$ & 174.91 & 174.92 & -0.01 \\
$\mathrm{Z} \rightarrow$ ee & 174.91 & 174.89 & 0.01 \\
$\mathrm{Z} \rightarrow \mu \mu$ & 174.87 & 174.91 & -0.02 \\
$\mathrm{Z} \rightarrow \tau \tau$ & 174.97 & 174.88 & 0.05 \\
All & 174.78 & 174.99 & -0.10 \\
\hline Quad Sum & Shifted Up & Shifted Down & 0.35 \\
\hline Nominal & \multicolumn{3}{|c}{$174.75 \pm 0.38$} \\
\hline
\end{tabular}

Table 31: Likelihood only mass distribution means and the systematic uncertainties for each of the shifted backgrounds and the sum in quadrature of the systematic uncertainties. 


\begin{tabular}{l|c|c|r}
\hline Background & Mass Mean & Mass Mean & Diff/2 \\
\hline \hline Fakes & 174.62 & 175.17 & -0.28 \\
WW & 174.75 & 175.00 & -0.13 \\
WZ & 174.82 & 174.90 & -0.04 \\
ZZ & 174.78 & 174.95 & -0.09 \\
W & 174.87 & 174.87 & 0.00 \\
$\mathrm{Z} \rightarrow$ ee & 174.98 & 174.75 & 0.12 \\
$\mathrm{Z} \rightarrow \mu \mu$ & 177.25 & 177.32 & -0.03 \\
$\mathrm{Z} \rightarrow \tau \tau$ & 174.92 & 174.84 & 0.04 \\
All & 174.78 & 174.92 & -0.07 \\
\hline Quad Sum & Shifted Up & Shifted Down & 0.35 \\
\hline Nominal & \multicolumn{3}{|c}{$174.71 \pm 0.33$} \\
\hline
\end{tabular}

Table 32: Likelihood added mass distribution means and the systematic uncertainties for each of the shifted backgrounds and the sum in quadrature of the systematic uncertainties.

\begin{tabular}{l|c|c|r}
\hline Background & Mass Mean & Mass Mean & Diff/2 \\
\hline \hline Fakes & 177.01 & 177.33 & -0.16 \\
WW & 177.22 & 177.27 & -0.03 \\
WZ & 177.13 & 177.27 & -0.07 \\
$\mathrm{ZZ}$ & 177.07 & 177.32 & -0.13 \\
$\mathrm{~W} \gamma$ & 177.17 & 177.25 & -0.04 \\
$\mathrm{Z} \rightarrow$ ee & 176.86 & 177.51 & -0.33 \\
$\mathrm{Z} \rightarrow \mu \mu$ & 177.30 & 177.14 & 0.08 \\
$\mathrm{Z} \rightarrow \tau \tau$ & 177.27 & 177.18 & 0.04 \\
All & 177.14 & 177.24 & -0.05 \\
\hline Quad Sum & Shifted Up & Shifted Down & 0.41 \\
\hline Nominal & \multicolumn{3}{|c}{$174.44 \pm 0.38$} \\
\hline
\end{tabular}

Table 33: 2 leading jets mass distribution means and the systematic uncertainties for each of the shifted backgrounds and the sum in quadrature of the systematic uncertainties. 


\begin{tabular}{l|c|c|r}
\hline Background & Mass Mean & Mass Mean & Diff/2 \\
\hline \hline Fakes & 173.99 & 174.47 & -0.24 \\
WW & 174.09 & 174.37 & -0.14 \\
WZ & 174.18 & 174.24 & -0.03 \\
$\mathrm{ZZ}$ & 174.11 & 174.28 & -0.09 \\
W $\gamma$ & 174.21 & 174.22 & -0.01 \\
$\mathrm{Z} \rightarrow$ ee & 174.27 & 174.14 & 0.07 \\
$\mathrm{Z} \rightarrow \mu \mu$ & 174.20 & 174.19 & 0.01 \\
$\mathrm{Z} \rightarrow \tau \tau$ & 174.32 & 174.13 & 0.09 \\
All & 174.17 & 174.31 & -0.07 \\
\hline Quad Sum & Shifted Up & Shifted Down & 0.32 \\
\hline Nominal & \multicolumn{3}{|c}{$174.93 \pm 0.33$} \\
\hline
\end{tabular}

Table 34: 2 leading jets mass distribution means and the systematic uncertainties for each of the shifted backgrounds and the sum in quadrature of the systematic uncertainties.

The systematic errors due to different background fractions are:

$$
\begin{aligned}
\sigma_{B G}^{\text {sys }}(L) & = \pm 0.38 G e V & \sigma_{B G}^{\text {sys }}(L+) & = \pm 0.35 G e V \\
\sigma_{B G}^{s y s}(2 L J) & = \pm 0.41 G e V & \sigma_{B G}^{\text {sys }}(2 L J+) & = \pm 0.33 G e V
\end{aligned}
$$




\section{$6.7 \quad b$-Jet Energy Scale}

To account for differences in the $b$-jet energy scale in the MC data and the real data, a systematic uncertainty is assigned. To calculate this uncertainty, the $b$-jets in the MC data are identified from the truth table and their energies are shifted up and down by $1 \%$. If the nominal value lies in between the resulting mass values from shifting the energies, then the half of the difference of the two mass values is calculated. In order to relate this $1 \%$ effect to the appropriate level, this difference needs to be scaled by $0.2 \%$. This is an estimate of how 1-sigma changes to $b$-fragmentation, $b$-semi-leptonic branching ratios, and $b$-color-flow affect the $b$-jet energy scale. The larger scaled value is the systematic uncertainty.

In addition to moving the $b$-jet energies, there are two other systematics that need to be calculated. The first is related to the semi-leptonic branching ratios. Both of the $\mathrm{b}$ and $\mathrm{c}$ branching ratios need to be moved up and down by one sigma. The other is related to $b$ fragmentation where a $b$-hadron is produced when a $b$-quark is produced. The quantity of interest is the fraction of momentum that the $b$-hadron carries away from the $b$-quark. For these systematics, the mass distributions are re-weighted in a similar way to the PDF study.

Once all three of these systematic uncertainties are calculated, they are added in quadrature to come up with the total systematic uncertainty from the $b$-jet energy scale.

The systematic errors due to the $b$-Jet Energy Scale are:

$$
\begin{aligned}
& \sigma_{\text {bJes }}^{\text {sys }}(L)= \pm 0.38 \mathrm{GeV} \quad \sigma_{\text {bJes }}^{\text {sys }}(L+)= \pm 0.33 \mathrm{GeV} \\
& \sigma_{b J e s}^{\text {sys }}(2 L J)= \pm 0.38 G e V \quad \sigma_{b J e s}^{\text {sys }}(2 L J+)= \pm 0.33 G e V
\end{aligned}
$$

\begin{tabular}{l|c|c|c}
\hline Systematic & Mass Mean & Mass Mean & Syst. \\
\hline \hline b-JES & $176.51 \pm 0.38$ & $173.76 \pm 0.38$ & 0.28 \\
Branching Ratio & $174.94 \pm 0.78$ & $174.91 \pm 0.77$ & 0.03 \\
Fragmentation & $174.89 \pm 0.77$ & $174.89 \pm 0.78$ & -0.00 \\
\hline Quad Sum & Shifted Up (ADO) & Shifted Down (SLD) & 0.28 \\
\hline Nominal & \multicolumn{2}{|c}{$174.75 \pm 0.38$} \\
\hline
\end{tabular}

Table 35: Likelihood only mass distribution means and the uncertainty on the means for the $b$-Jet systematic studies. 


\begin{tabular}{l|c|c|c}
\hline Systematic & Mass Mean & Mass Mean & Syst. \\
\hline \hline b-JES & $176.64 \pm 0.35$ & $174.12 \pm 0.35$ & 0.25 \\
Branching Ratio & $174.88 \pm 0.68$ & $174.88 \pm 0.68$ & 0.00 \\
Fragmentation & $174.86 \pm 0.68$ & $174.87 \pm 0.68$ & -0.01 \\
\hline Quad Sum & Shifted Up (ADO) & Shifted Down (SLD) & 0.25 \\
\hline Nominal & \multicolumn{2}{|c}{$174.71 \pm 0.33$} \\
\hline
\end{tabular}

Table 36: Likelihood added mass distribution means and the uncertainty on the means for the b-Jet systematic studies.

\begin{tabular}{l|c|c|c}
\hline Systematic & Mass Mean & Mass Mean & Syst. \\
\hline \hline b-JES & $176.33 \pm 0.38$ & $173.27 \pm 0.39$ & 0.31 \\
Branching Ratio & $177.18 \pm 0.99$ & $177.16 \pm 0.98$ & 0.02 \\
Fragmentation & $177.20 \pm 0.98$ & $177.16 \pm 0.98$ & 0.04 \\
\hline Quad Sum & Shifted Up (ADO) & Shifted Down (SLD) & 0.31 \\
\hline Nominal & \multicolumn{2}{|c}{$174.44 \pm 0.38$} \\
\hline
\end{tabular}

Table 37: 2 leading jets mass distribution means and the uncertainty on the means for the $b$-Jet systematic studies.

\begin{tabular}{l|c|c|r}
\hline Systematic & Mass Mean & Mass Mean & Syst. \\
\hline \hline b-JES & $176.10 \pm 0.35$ & $173.56 \pm 0.35$ & 0.25 \\
Branching Ratio & $174.23 \pm 0.75$ & $174.19 \pm 0.74$ & 0.04 \\
Fragmentation & $174.20 \pm 0.74$ & $174.22 \pm 0.74$ & -0.01 \\
\hline Quad Sum & Shifted Up (ADO) & Shifted Down (SLD) & 0.26 \\
\hline Nominal & \multicolumn{2}{|c}{$174.93 \pm 0.33$} \\
\hline
\end{tabular}

Table 38: 2 leading jets added mass distribution means and the uncertainty on the means for the $b$-Jet systematic studies. 


\subsection{Lepton $P_{T}$}

It has been shown that the MC data has an energy scale for electrons that is $0.5 \%$ higher than that in the real data. To account for this, a systematic uncertainty has been assigned. For a given MC mass sample, the energies of the leptons have been shifted up and down by $1 \%$. The systematic uncertainty is half of the difference between the samples with their energies shifted, if the nominal value lies between them.

\begin{tabular}{l|c|c|c|c}
\hline MC Sample & Likelihood & L Added & 2LJ & 2LJ Added \\
\hline \hline Nominal & $174.75 \pm 0.38$ & $174.71 \pm 0.33$ & $174.44 \pm 0.38$ & $174.93 \pm 0.33$ \\
Shifted Down & $174.26 \pm 0.42$ & $174.67 \pm 0.39$ & $173.79 \pm 0.42$ & $174.07 \pm 0.39$ \\
Shifted Up & $174.97 \pm 0.38$ & $175.15 \pm 0.35$ & $174.74 \pm 0.38$ & $174.62 \pm 0.35$ \\
\hline Systematic Shift & 0.35 & 0.24 & 0.48 & -0.43 \\
\hline
\end{tabular}

Table 39: Mass distribution means and the uncertainty on the means for the shifted lepton energy systematic study.

The systematic errors due to Lepton $P_{T}$ are:

$$
\begin{aligned}
\sigma_{L P T}^{s y s}(L) & = \pm 0.38 G e V & \sigma_{L P T}^{s y s}(L+) & = \pm 0.33 G e V \\
\sigma_{L P T}^{s y s}(2 L J) & = \pm 0.48 G e V & \sigma_{L P T}^{s y s}(2 L J+) & = \pm 0.43 G e V
\end{aligned}
$$

\subsection{Pile-Up}

A systematic uncertainty to account for the possibility that an event has multiple $p \bar{p}$ interactions needs to be assigned. There are two pieces that make up this systematic uncertainty, the first is due to the known mismodeling of the MC luminosity profile. The second part is due to possible mismodeling of the minbias events. The larger of the two parts will be quoted as the systematic uncertainty due to pile-up.

The MC datasets used in this analysis were generated with luminosity profiles that correspond to the first $1.2 \mathrm{fb}^{-1}$ of data. To determine the systematic uncertainty due to the known mismodeling of the luminosity profile, events from the MC data sample with a set mass of $175 \mathrm{GeV}$ were separated by the number of interactions, PEs were made for each set of events, 
and the mass mean from each set of events was plotted against the number of interactions (Figure 44). The slope of the line fitted to these point, $m_{P U}$, multiplied by the difference of the average number of interactions of the real data and $\mathrm{MC}$, will be the systematic uncertainty assigned to the known mismodeling.

$$
\sigma_{P U_{\text {known }}}^{\text {sys }}=m_{P U}(<N I N T>(\text { data })-<N I N T>(M C))
$$

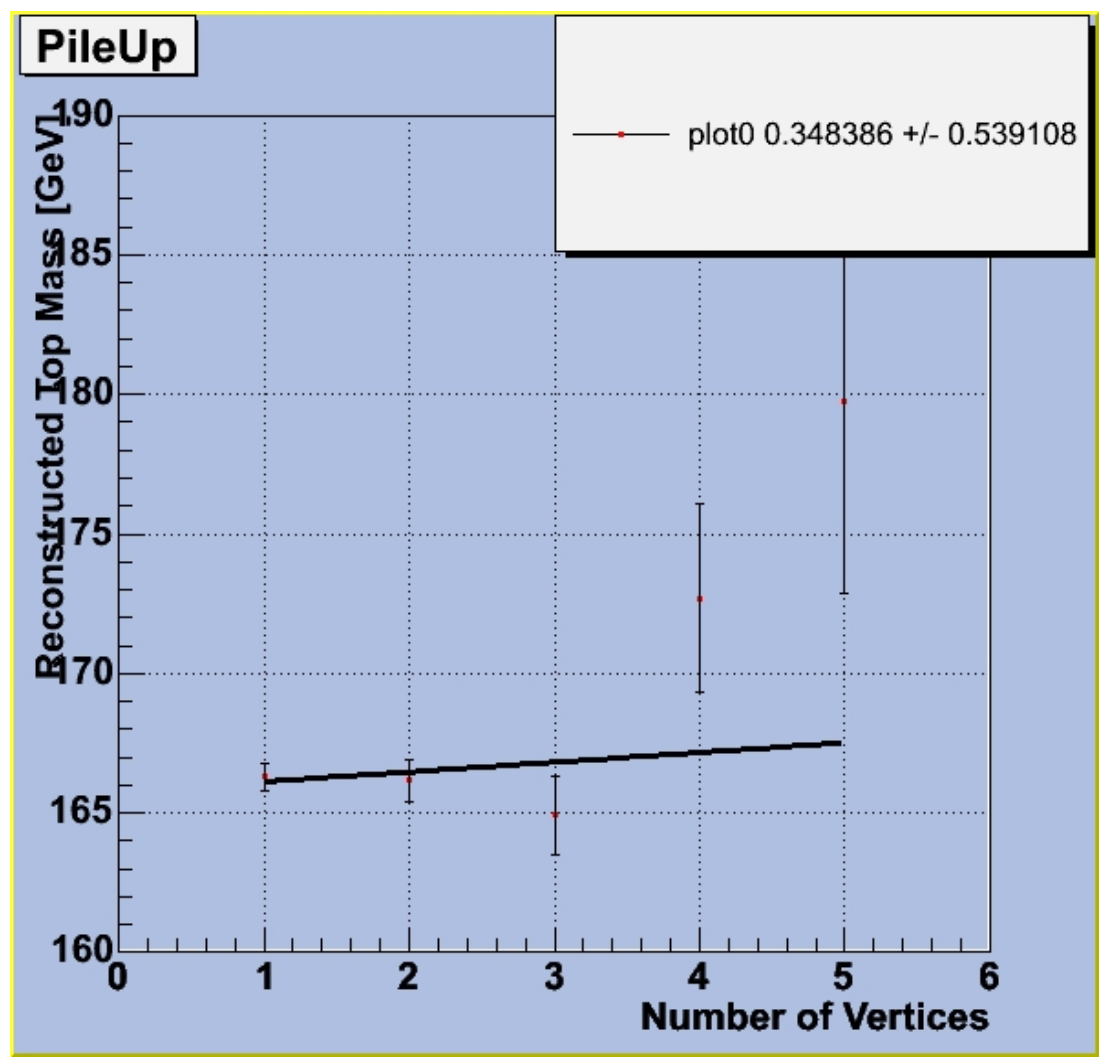

Figure 44: Mass mean as a function of the number of interactions per event.

The conversion of luminosity to minbias events in the MC was wrong by about 20\%. Even if the luminocity profile was correct, this would cause a systematic shift. According to the CDF Top Group, "The observed jet response slope after jet corrections is $250 \mathrm{MeV} /$ jet/vertex. The nominal L4 systematic is $107 \mathrm{MeV} /$ jet/vertex. So we can estimate the importance of this order of mismodeling by scaling up the L4 systematic by a factor of 2.3. An additional complication is that the L4 systematic is usually evaluated on e.g. ttop75, which as we know has the incorrect luminosity profile. The L4 correction/systematic goes as the number of vertices minus one, so the scaling factor becomes (Eq. 81)." [17] 


$$
\sigma_{P U_{\text {Unknown }}^{\text {sys }}}^{\text {sy }}=2.3 \frac{<N I N T(\text { data })>-1}{<N I N T(M C)>-1} \sigma_{L 4}^{\text {sys }}
$$

\begin{tabular}{c|cccc}
\hline Variable & Likelihood & L Added & 2LJ & 2LJ Added \\
\hline \hline$<N I N T>($ data $)$ & 1.90 & 1.90 & 1.90 & 1.90 \\
$<N I N T>(M C)$ & 1.62 & 1.62 & 1.63 & 1.63 \\
$m_{P U}$ & 0.35 & & & \\
$\sigma_{m_{P U}}$ & 0.54 & & & \\
$\sigma_{L 4}^{\text {sys }}$ & -0.22 & -0.28 & -0.28 & -0.39 \\
\hline
\end{tabular}

Table 40: The values of the various variables that go into calculating the systematic uncertainty due to pile-up.

$$
\begin{aligned}
\sigma_{P U_{K n o w n}}^{\text {sys }} & = \pm 0.10 \mathrm{GeV} \\
\sigma_{P U_{U n k n o w n}}^{\text {sys }}(L) & = \pm 0.73 \mathrm{GeV} \\
\sigma_{P U_{U \text { Unknown }}}^{\text {sys }}(L+) & = \pm 0.93 \mathrm{GeV} \\
\sigma_{P U_{\text {Unknown }}}^{\text {sys }}(2 \mathrm{LJ}) & = \pm 0.92 \mathrm{GeV} \\
\sigma_{P U_{\text {Unknown }}^{\text {sys }}}^{\text {sys }}(2 L J+) & = \pm 1.28 \mathrm{GeV}
\end{aligned}
$$

The systematic errors due to Pile-up are:

$$
\begin{aligned}
& \sigma_{P U}^{s y s}(L)= \pm 0.73 G e V \quad \sigma_{P U}^{s y s}(L+)= \pm 0.93 G e V \\
& \sigma_{P U}^{\text {sys }}(2 L J)= \pm 0.92 \mathrm{GeV} \quad \sigma_{P U}^{\text {sys }}(2 L J+)= \pm 1.28 \mathrm{GeV}
\end{aligned}
$$

\subsection{Color Reconnection}

In order to account for a possible bias due to Color Reconnection (CR), two MC samples were generated. The first sample, ctopsd, was generated with $M_{t}=172.5 \mathrm{GeV}$ and set to tune Apro, which is very similar to the tune for the nominal MC generations. The other sample, ctopse, is set to the same $M_{t}$ and tune ACRpro, which includes CR. To calculate the systematic uncertainty, the difference in mass mean is calculated between the two samples. 


\begin{tabular}{lcccc}
\hline MC Sample & Likelihood & L Added & 2LJ & 2LJ Added \\
\hline \hline ctopsd & $171.16 \pm 0.53$ & $172.42 \pm 0.50$ & $170.26 \pm 0.53$ & $171.58 \pm 0.50$ \\
ctopse & $172.33 \pm 0.52$ & $172.19 \pm 0.50$ & $171.68 \pm 0.53$ & $171.17 \pm 0.50$ \\
\hline \hline Shift & $-1.18 \pm 0.74$ & $0.24 \pm 0.71$ & $-1.42 \pm 0.75$ & $0.40 \pm 0.71$ \\
\hline
\end{tabular}

Table 41: Mass distribution means and the uncertainty on the means for the two color reconnection samples as well as the difference.

The systematic errors due to Color Reconnection are:

$$
\begin{aligned}
\sigma_{C R}^{\text {sys }}(L) & = \pm 1.18 G e V & \sigma_{C R}^{\text {sys }}(L+) & = \pm 0.24 G e V \\
\sigma_{C R}^{\text {sys }}(2 L J) & = \pm 1.42 G e V & \sigma_{C R}^{\text {sys }}(2 L J+) & = \pm 0.40 G e V
\end{aligned}
$$

\subsection{Systematic Summary}

Finally, to calculate the total systematic uncertainty to the top mass from this analysis, each of the individual uncertainties are added together in quadrature.

The Total Systematic Uncertainties from all sources are

$$
\begin{aligned}
& \sigma_{T O T}^{\text {sys }}(L)= \pm 2.25 G e V \quad \sigma_{T O T}^{\text {sys }}(L+)= \pm 2.04 G e V \\
& \sigma_{T O T}^{\text {sys }}(2 L J)= \pm 2.42 G e V \quad \sigma_{T O T}^{\text {sys }}(2 L J+)= \pm 1.92 G e V
\end{aligned}
$$




\begin{tabular}{lcccc}
\hline Source & Likelihood & Likelihood + & 2LJ & 2LJ+ \\
\hline \hline Jet Energy Scale & $\pm 1.25 \mathrm{GeV}$ & $\pm 1.42 \mathrm{GeV}$ & $\pm 1.10 \mathrm{GeV}$ & $\pm 1.09 \mathrm{GeV}$ \\
PDF & $\pm 0.78 \mathrm{GeV}$ & $\pm 0.74 \mathrm{GeV}$ & $\pm 0.34 \mathrm{GeV}$ & $\pm 0.30 \mathrm{GeV}$ \\
ISR/FSR & $\pm 0.38 \mathrm{GeV}$ & $\pm 0.46 \mathrm{GeV}$ & $\pm 0.87 \mathrm{GeV}$ & $\pm 0.33 \mathrm{GeV}$ \\
MC Generator & $\pm 0.62 \mathrm{GeV}$ & $\pm 0.33 \mathrm{GeV}$ & $\pm 0.61 \mathrm{GeV}$ & $\pm 0.33 \mathrm{GeV}$ \\
Background & $\pm 0.38 \mathrm{GeV}$ & $\pm 0.35 \mathrm{GeV}$ & $\pm 0.41 \mathrm{GeV}$ & $\pm 0.33 \mathrm{GeV}$ \\
b-Jet Energy Scale & $\pm 0.38 \mathrm{GeV}$ & $\pm 0.33 \mathrm{GeV}$ & $\pm 0.38 \mathrm{GeV}$ & $\pm 0.33 \mathrm{GeV}$ \\
Lepton P & $\pm 0.38 \mathrm{GeV}$ & $\pm 0.33 \mathrm{GeV}$ & $\pm 0.48 \mathrm{GeV}$ & $\pm 0.43 \mathrm{GeV}$ \\
Pile-up & $\pm 0.73 \mathrm{GeV}$ & $\pm 0.93 \mathrm{GeV}$ & $\pm 0.92 \mathrm{GeV}$ & $\pm 1.28 \mathrm{GeV}$ \\
Color Reconnection & $\pm 1.18 \mathrm{GeV}$ & $\pm 0.24 \mathrm{GeV}$ & $\pm 1.42 \mathrm{GeV}$ & $\pm 0.40 \mathrm{GeV}$ \\
\hline \hline Total Systematic Uncertainty & $\pm 2.25 \mathrm{GeV}$ & $\pm 2.04 \mathrm{GeV}$ & $\pm 2.42 \mathrm{GeV}$ & $\pm 1.92 \mathrm{GeV}$ \\
\hline
\end{tabular}

Table 42: Systematic errors from various sources 


\section{Chapter 7 - b-Tagging}

\subsection{Introduction}

A powerful tool for reducing the background for top events is $b$-tagging. $b$-tagging identifies (tags) jets that came from $b$-quarks by using their long lifetimes and expected decay signatures. The technique was originally developed for top searches in the lepton + jets channel where it is crucial to be able to distinguish the signal from backgrounds such as $\mathrm{W}+$ jets. Due to the low levels of background inherent in the di-lepton channel, $b$-tagging is not required; however, it is very useful for verifying that the candidate di-lepton events represent the expected signal. Figure 45[18] shows that $b$-tagging works, and for the purposes of this analysis is treated as another cut.

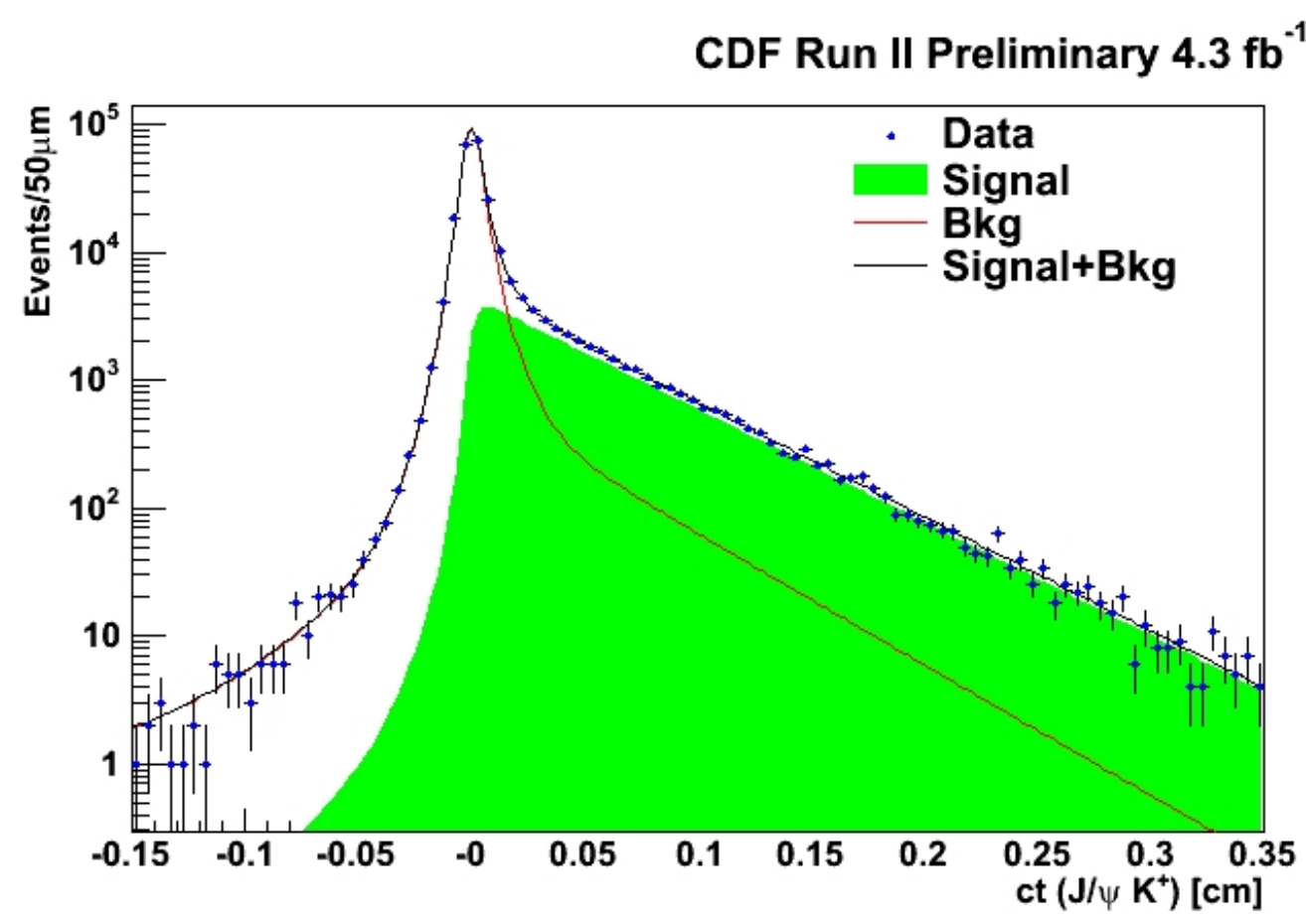

Figure 45: This figure shows the proper decay lengths of the $b$-quark into $\mathrm{J} / \psi K^{+}$. 


\subsection{Background}

When $b$-tagging is applied to the di-lepton channel, the backgrounds are significantly reduced while the signal is mostly preserved. This gives a much higher signal to background ratio and a much cleaner data sample. Table 43 lists the expected number of events in the data for each of the backgrounds and $t \bar{t}$.

\begin{tabular}{lcc}
\hline Source & Data Set & Number of Events \\
\hline \hline Fakes & W+jets data & $2.37 \pm 0.69$ \\
$\mathrm{DY} \rightarrow e e+\mu \mu$ & zewkad + zewk9m & $0.84 \pm 0.14$ \\
$\mathrm{WW}$ & itopww & $0.23 \pm 0.04$ \\
$\mathrm{DY} \rightarrow \tau \tau$ & zewkat & $1.04 \pm 0.20$ \\
$\mathrm{WZ}$ & itopwz & $0.04 \pm 0.01$ \\
$\mathrm{ZZ}$ & itopzz & $0.09 \pm 0.07$ \\
\hline Total Background & ttop75 & $4.61 \pm 0.74$ \\
$t \bar{t}(\sigma=6.7 \mathrm{pb})$ & & $54.66 \pm 4.16$ \\
\hline Total SM Expectation & & $62.27 \pm 4.22$ \\
\hline \hline Events Observed in Data & \\
\hline
\end{tabular}

Table 43: Signal and background event expectations predicted by the Standard Model with $b$-tagging.

\subsection{Mapping Functions}

New mapping functions are required due to the new signal to background ratio. Figures 46 through 49 show the same set of mapping functions used in Chapter 6 with the addition of $b$-tagging.

$$
\begin{array}{lc}
M_{t}^{\text {raw }}=0.750 \times M_{t}^{\text {corr }}+36.364 & \text { (Likelihood Only) } \\
M_{t}^{\text {raw }}=0.747 \times M_{t}^{\text {corr }}+35.592 & \text { (Likelihood Added) } \\
M_{t}^{\text {raw }}=0.752 \times M_{t}^{\text {corr }}+36.490 & \text { (2 Leading Jets) } \\
M_{t}^{\text {raw }}=0.754 \times M_{t}^{\text {corr }}+34.960 & \text { (2 Leading Jets Added) }
\end{array}
$$



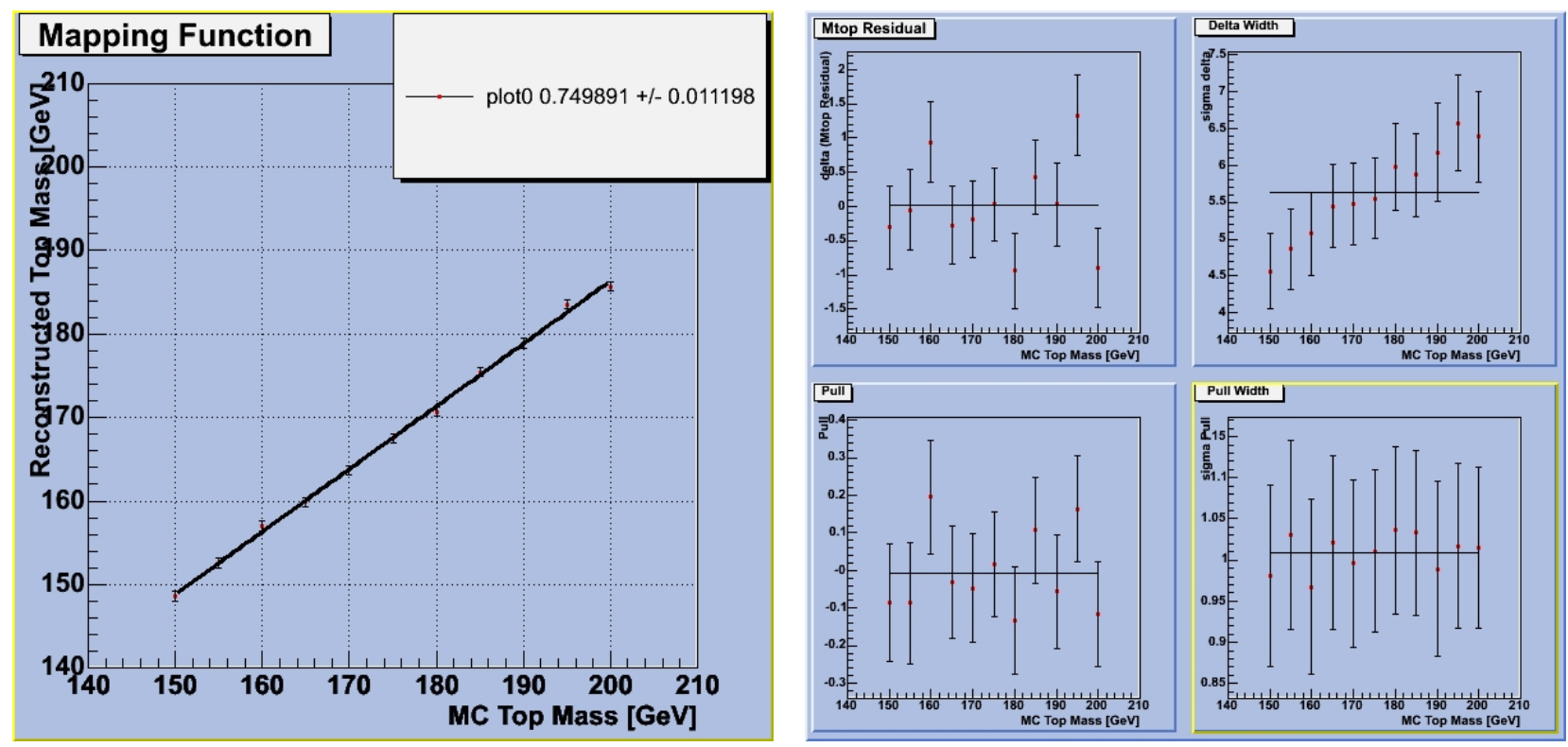

Figure 46: Standard set of plots for the signal and background data samples with $b$-tagging where the most probable combination is selected.
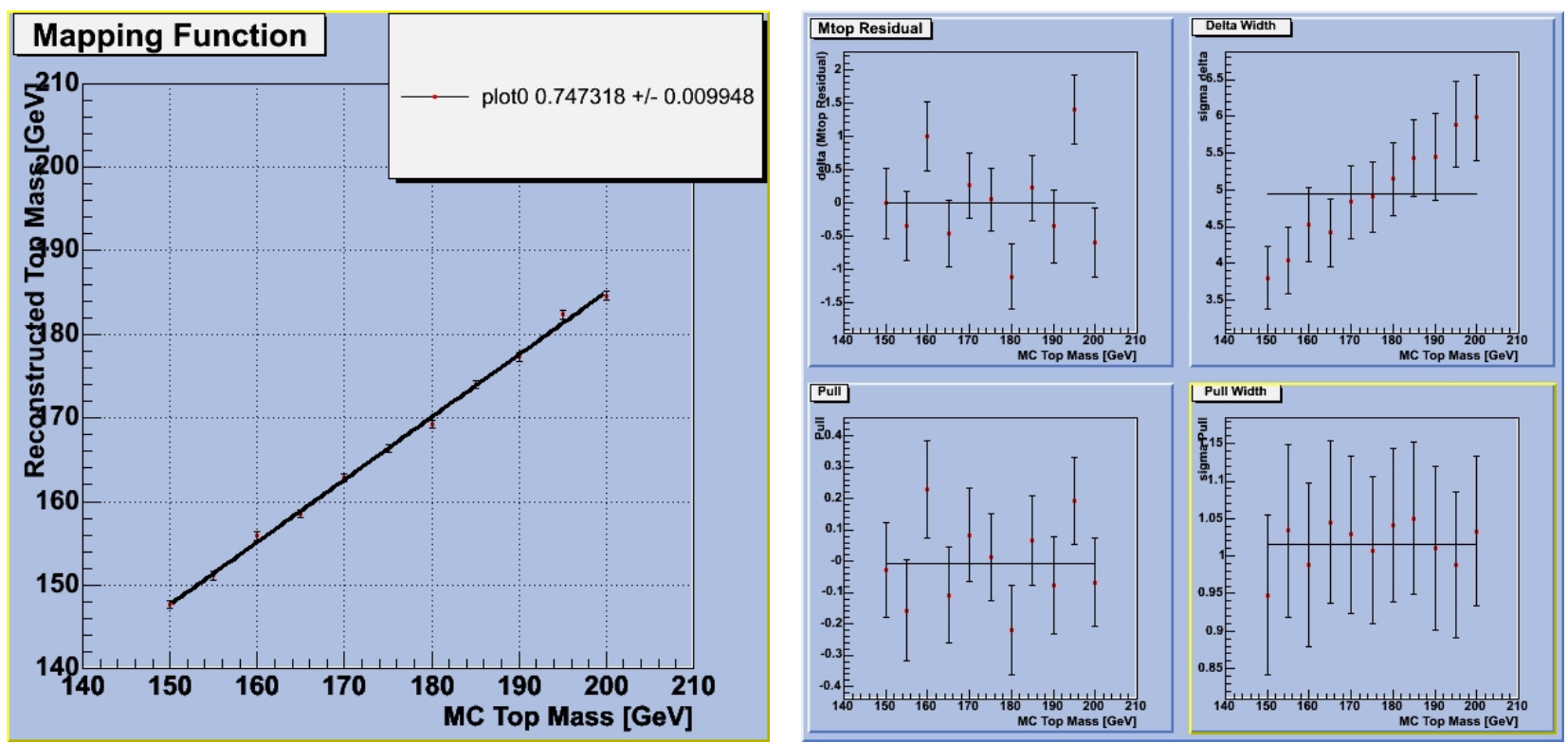

Figure 47: Standard set of plots for the signal and background data samples with $b$-tagging where the most probable combination is added to its "twin." 

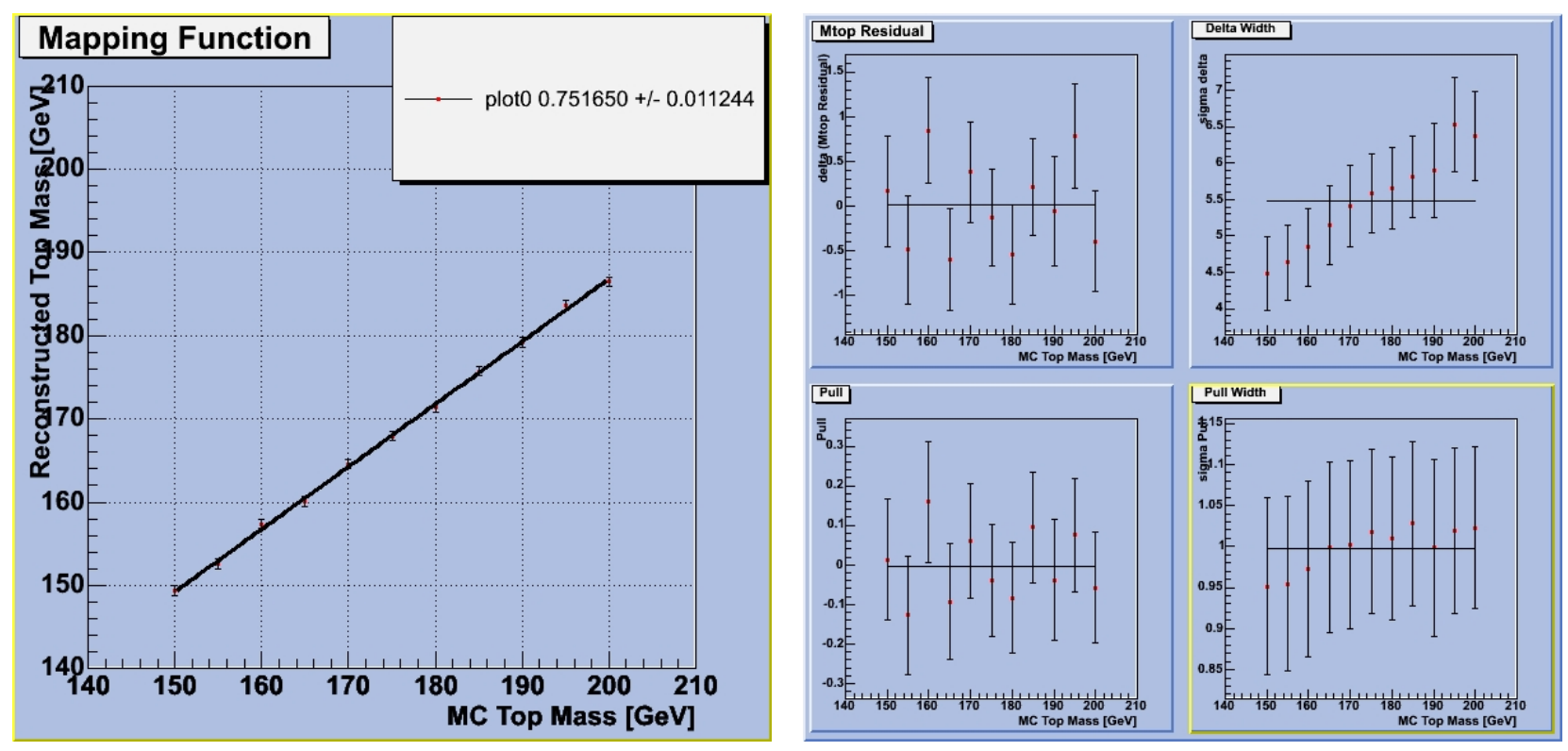

Figure 48: Standard set of plots for the signal and background data samples with $b$-tagging where the most probable combination is selected out of the combinations which contain both of the leading jets. 

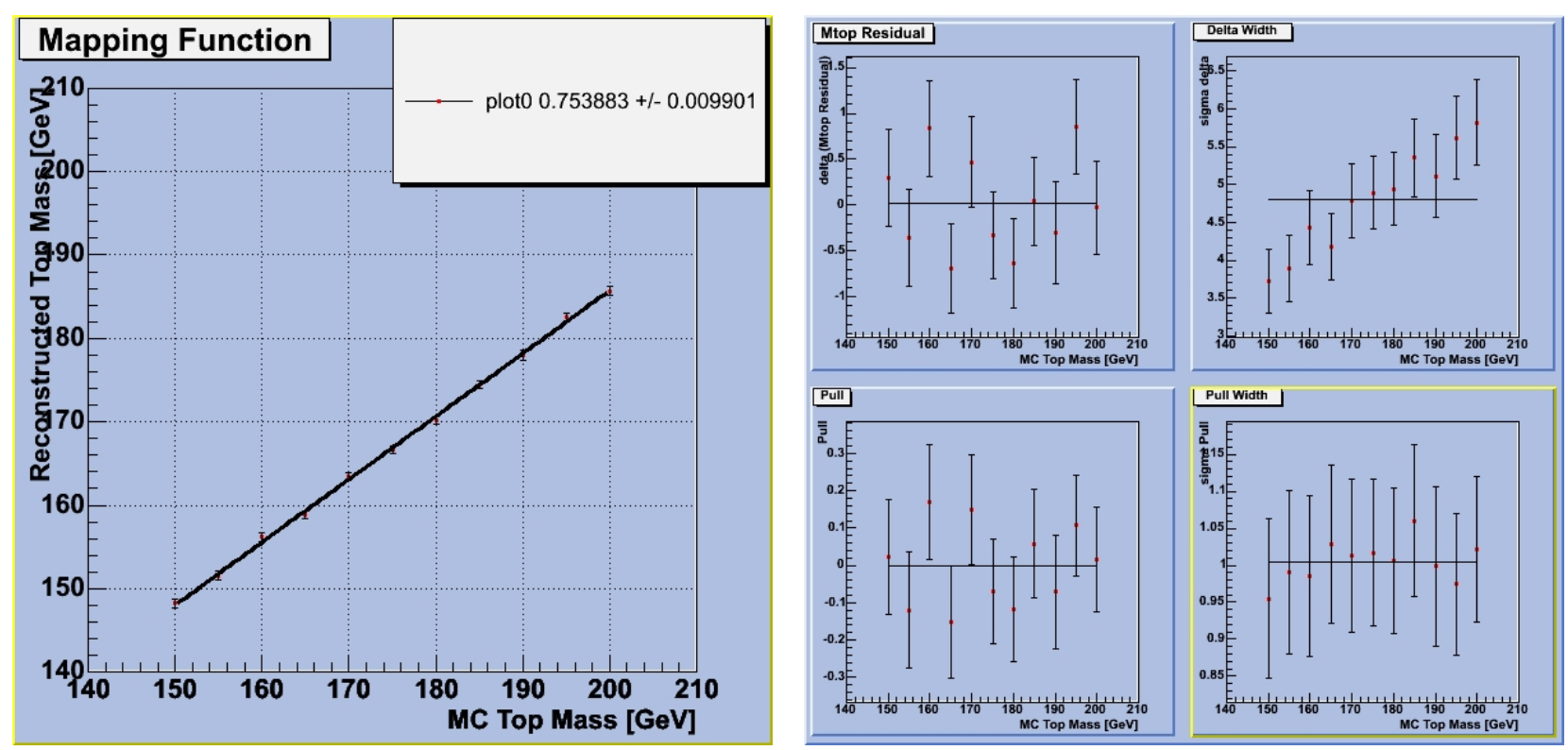

Figure 49: Standard set of plots for the signal and background data samples with $b$-tagging where the most probable combination is selected out of the combinations which contain both of the leading jets and is added to its "twin."

\subsection{Statistical Errors}

The statistical error on the measurement will need to be corrected in the same way as described in Section 5.7, only with new values. Table 44 lists those values.

\begin{tabular}{lcc}
\hline Combination Choice & Slope & RMS Correction \\
\hline \hline Likelihood Only & 0.750 & 2.77 \\
Likelihood Added & 0.747 & 2.27 \\
2 Leading Jets & 0.752 & 2.73 \\
2 Leading Jets Added & 0.754 & 2.22 \\
\hline
\end{tabular}

Table 44: Correction factors to the width of the top mass joint distribution with $b$-tagging.

\subsection{Systematic Errors}

The systematic errors that will be used for this analysis with $b$-tagging will need to be calculated for each of the four strategies of choosing combinations of lepton and jets. Subsections 7.5.1 
through 7.5.10 and the tables contained within show the results of the systematic studies done with $b$-tagging.

\subsubsection{Jet Energy Scale}

\begin{tabular}{l|rr|rr|r}
\hline Correction & Mass Mean & Stat. & Mass Mean & Stat. & Syst. \\
\hline \hline All at Once & 176.72 & \pm 0.54 & 173.67 & \pm 0.54 & 1.53 \\
\hline Level 1 & 174.52 & \pm 1.04 & 175.61 & \pm 1.04 & -0.55 \\
Level 4 & 174.68 & \pm 1.04 & 175.26 & \pm 1.05 & -0.29 \\
Level 5 & 174.87 & \pm 1.05 & 174.93 & \pm 1.05 & -0.03 \\
Level 6 & 175.16 & \pm 1.08 & 174.93 & \pm 1.06 & 0.11 \\
Level 7 & 175.31 & \pm 1.06 & 174.43 & \pm 1.07 & 0.44 \\
Level 8 & 174.41 & \pm 1.04 & 175.58 & \pm 1.05 & -0.58 \\
\hline Quad Sum & \multicolumn{7}{|c|}{ Shifted Up $174.75 \pm 0.48$} \\
\hline Nominal & \multicolumn{7}{|c}{ Shifted Down } & 0.97 \\
\hline
\end{tabular}

Table 45: Likelihood only mass distribution means, uncertainty on the means, and systematic uncertainties from each of the jet energy correction levels. Additionally, the sum in quadrature for all the individual levels.

\begin{tabular}{l|rr|cr|r}
\hline Correction & Mass Mean & Stat. & Mass Mean & Stat. & Syst. \\
\hline \hline All at Once & 176.81 & \pm 0.48 & 174.16 & \pm 0.48 & 1.33 \\
\hline Level 1 & 175.31 & \pm 0.94 & 175.98 & \pm 0.95 & -0.33 \\
Level 4 & 175.38 & \pm 0.94 & 175.71 & \pm 0.94 & -0.17 \\
Level 5 & 175.48 & \pm 0.94 & 175.44 & \pm 0.94 & 0.02 \\
Level 6 & 176.07 & \pm 0.96 & 175.14 & \pm 0.95 & 0.47 \\
Level 7 & 176.06 & \pm 0.95 & 174.76 & \pm 0.96 & 0.65 \\
Level 8 & 175.07 & \pm 0.94 & 175.90 & \pm 0.94 & -0.41 \\
\hline Quad Sum & \multicolumn{7}{|c|}{ Shifted Up } & Shifted Down & 0.97 \\
\hline Nominal & \multicolumn{7}{|c}{$175.08 \pm 0.42$} \\
\hline
\end{tabular}

Table 46: Likelihood added mass distribution means, uncertainty on the means, and systematic uncertainties from each of the jet energy correction levels. Additionally, the sum in quadrature for all the individual levels. 


\begin{tabular}{l|rr|rr|r}
\hline Correction & Mass Mean & Stat. & Mass Mean & Stat. & Syst. \\
\hline \hline All at Once & 176.89 & \pm 0.54 & 173.65 & \pm 0.55 & 1.62 \\
\hline Level 1 & 174.98 & \pm 1.06 & 176.27 & \pm 1.07 & -0.64 \\
Level 4 & 175.24 & \pm 1.06 & 175.75 & \pm 1.06 & -0.25 \\
Level 5 & 175.48 & \pm 1.06 & 175.52 & \pm 1.06 & -0.02 \\
Level 6 & 175.63 & \pm 1.09 & 175.26 & \pm 1.09 & 0.19 \\
Level 7 & 176.11 & \pm 1.08 & 175.20 & \pm 1.09 & 0.46 \\
Level 8 & 174.95 & \pm 1.06 & 175.94 & \pm 1.07 & -0.49 \\
\hline Quad Sum & Shifted Up & Shifted Down & 0.98 \\
\hline Nominal & \multicolumn{5}{|c}{$174.48 \pm 0.48$} \\
\hline
\end{tabular}

Table 47: 2 leading jets mass distribution means, uncertainty on the means, and systematic uncertainties from each of the jet energy correction levels. Additionally, the sum in quadrature for all the individual levels.

\begin{tabular}{l|rr|cr|r}
\hline Correction & Mass Mean & Stat. & Mass Mean & Stat. & Syst. \\
\hline \hline All at Once & 176.40 & \pm 0.47 & 173.85 & \pm 0.48 & 1.28 \\
\hline Level 1 & 174.98 & \pm 0.93 & 175.95 & \pm 0.94 & -0.48 \\
Level 4 & 175.14 & \pm 0.93 & 175.48 & \pm 0.93 & -0.17 \\
Level 5 & 175.19 & \pm 0.93 & 175.21 & \pm 0.93 & -0.01 \\
Level 6 & 175.50 & \pm 0.95 & 175.05 & \pm 0.94 & 0.22 \\
Level 7 & 175.85 & \pm 0.94 & 174.77 & \pm 0.95 & 0.54 \\
Level 8 & 174.84 & \pm 0.93 & 175.66 & \pm 0.94 & -0.41 \\
\hline Quad Sum & Shifted Up & Shifted Down & 0.88 \\
\hline Nominal & \multicolumn{5}{|c}{$175.10 \pm 0.41$} \\
\hline
\end{tabular}

Table 48: 2 leading jets added mass distribution means, uncertainty on the means, and systematic uncertainties from each of the jet energy correction levels. Additionally, the sum in quadrature for all the individual levels. 
The systematic errors due to the Jet Energy Scale are:

$$
\begin{aligned}
\sigma_{J E S}^{\text {sys }}(L) & = \pm 0.97 \mathrm{GeV} & \sigma_{J E S}^{\text {sys }}(L+) & = \pm 0.97 \mathrm{GeV} \\
\sigma_{J E S}^{\text {sys }}(2 L J) & = \pm 0.98 \mathrm{GeV} & \sigma_{J E S}^{\text {sys }}(2 L J+) & = \pm 0.88 \mathrm{GeV}
\end{aligned}
$$

\subsubsection{PDF}

\begin{tabular}{|lrr||lcr|}
\hline PDF Set & Mass Mean & Uncertainty & PDF Set & Mass Mean & Uncertainty \\
\hline \hline CTEQ5L & 175.00 & \pm 0.76 & MRST72 & 174.92 & \pm 0.76 \\
MRST75 & 175.00 & \pm 0.73 & CTEQ6L & 175.00 & \pm 0.76 \\
CTEQ6L1 & 174.98 & \pm 0.78 & CTEQ6M & 174.96 & \pm 0.76 \\
CTEQ6M1+ & 174.87 & \pm 0.75 & CTEQ6M1- & 174.81 & \pm 0.76 \\
CTEQ6M2+ & 174.79 & \pm 0.75 & CTEQ6M2- & 174.91 & \pm 0.75 \\
CTEQ6M3+ & 174.92 & \pm 0.75 & CTEQ6M3- & 174.94 & \pm 0.76 \\
CTEQ6M4+ & 174.84 & \pm 0.76 & CTEQ6M4- & 174.88 & \pm 0.75 \\
CTEQ6M5+ & 174.85 & \pm 0.75 & CTEQ6M5- & 174.95 & \pm 0.76 \\
CTEQ6M6+ & 174.84 & \pm 0.76 & CTEQ6M6- & 174.85 & \pm 0.75 \\
CTEQ6M7+ & 174.82 & \pm 0.75 & CTEQ6M7- & 174.89 & \pm 0.75 \\
CTEQ6M8+ & 174.96 & \pm 0.75 & CTEQ6M8- & 174.92 & \pm 0.76 \\
CTEQ6M9+ & 174.83 & \pm 0.75 & CTEQ6M9- & 174.90 & \pm 0.75 \\
CTEQ6M10+ & 174.89 & \pm 0.75 & CTEQ6M10- & 174.97 & \pm 0.76 \\
CTEQ6M11+ & 174.85 & \pm 0.76 & CTEQ6M11- & 174.84 & \pm 0.75 \\
CTEQ6M12+ & 174.77 & \pm 0.76 & CTEQ6M12- & 174.87 & \pm 0.76 \\
CTEQ6M13+ & 174.93 & \pm 0.75 & CTEQ6M13- & 174.90 & \pm 0.76 \\
CTEQ6M14+ & 174.84 & \pm 0.75 & CTEQ6M14- & 174.87 & \pm 0.75 \\
CTEQ6M15+ & 174.89 & \pm 0.77 & CTEQ6M15- & 175.04 & \pm 0.72 \\
CTEQ6M16+ & 174.82 & \pm 0.76 & CTEQ6M16- & 174.81 & \pm 0.76 \\
CTEQ6M17+ & 174.80 & \pm 0.76 & CTEQ6M17- & 174.86 & \pm 0.75 \\
CTEQ6M18+ & 174.92 & \pm 0.75 & CTEQ6M18- & 174.92 & \pm 0.76 \\
CTEQ6M19+ & 174.85 & \pm 0.74 & CTEQ6M19- & 174.87 & \pm 0.75 \\
CTEQ6M20+ & 174.85 & \pm 0.75 & CTEQ6M20- & 174.96 & \pm 0.76 \\
\hline
\end{tabular}

Table 49: Likelihood only mass distribution means and uncertainty on the means from each of the 46 PDFs 


\begin{tabular}{|lrr||lrr|}
\hline PDF Set & Mass Mean & Uncertainty & PDF Set & Mass Mean & Uncertainty \\
\hline \hline CTEQ5L & 175.15 & \pm 0.68 & MRST72 & 175.11 & \pm 0.67 \\
MRST75 & 175.16 & \pm 0.65 & CTEQ6L & 175.13 & \pm 0.68 \\
CTEQ6L1 & 175.13 & \pm 0.69 & CTEQ6M & 175.16 & \pm 0.67 \\
CTEQ6M1+ & 175.11 & \pm 0.67 & CTEQ6M1- & 175.07 & \pm 0.67 \\
CTEQ6M2+ & 175.14 & \pm 0.67 & CTEQ6M2- & 175.15 & \pm 0.67 \\
CTEQ6M3+ & 175.16 & \pm 0.67 & CTEQ6M3- & 175.22 & \pm 0.67 \\
CTEQ6M4+ & 175.06 & \pm 0.67 & CTEQ6M4- & 175.12 & \pm 0.66 \\
CTEQ6M5+ & 175.09 & \pm 0.66 & CTEQ6M5- & 175.15 & \pm 0.67 \\
CTEQ6M6+ & 175.10 & \pm 0.67 & CTEQ6M6- & 175.11 & \pm 0.67 \\
CTEQ6M7+ & 175.15 & \pm 0.67 & CTEQ6M7- & 175.15 & \pm 0.67 \\
CTEQ6M8+ & 175.19 & \pm 0.67 & CTEQ6M8- & 175.20 & \pm 0.67 \\
CTEQ6M9+ & 175.04 & \pm 0.67 & CTEQ6M9- & 175.13 & \pm 0.66 \\
CTEQ6M10+ & 175.10 & \pm 0.67 & CTEQ6M10- & 175.17 & \pm 0.67 \\
CTEQ6M11+ & 175.11 & \pm 0.68 & CTEQ6M11- & 175.10 & \pm 0.66 \\
CTEQ6M12+ & 175.12 & \pm 0.67 & CTEQ6M12- & 175.13 & \pm 0.67 \\
CTEQ6M13+ & 175.16 & \pm 0.67 & CTEQ6M13- & 175.20 & \pm 0.67 \\
CTEQ6M14+ & 175.06 & \pm 0.66 & CTEQ6M14- & 175.11 & \pm 0.66 \\
CTEQ6M15+ & 175.08 & \pm 0.68 & CTEQ6M15- & 175.27 & \pm 0.65 \\
CTEQ6M16+ & 175.10 & \pm 0.68 & CTEQ6M16- & 175.07 & \pm 0.67 \\
CTEQ6M17+ & 175.12 & \pm 0.67 & CTEQ6M17- & 175.14 & \pm 0.67 \\
CTEQ6M18+ & 175.16 & \pm 0.67 & CTEQ6M18- & 175.20 & \pm 0.67 \\
CTEQ6M19+ & 175.04 & \pm 0.66 & CTEQ6M19- & 175.11 & \pm 0.67 \\
CTEQ6M20+ & 175.09 & \pm 0.67 & CTEQ6M20- & 175.16 & \pm 0.67 \\
\hline
\end{tabular}

Table 50: Likelihood added mass distribution means and uncertainty on the means from each of the 46 PDFs 


\begin{tabular}{|lrr||lrr|}
\hline PDF Set & Mass Mean & Uncertainty & PDF Set & Mass Mean & Uncertainty \\
\hline \hline CTEQ5L & 176.07 & \pm 1.05 & MRST72 & 175.87 & \pm 1.03 \\
MRST75 & 175.89 & \pm 0.99 & CTEQ6L & 176.10 & \pm 1.04 \\
CTEQ6L1 & 176.10 & \pm 1.06 & CTEQ6M & 175.91 & \pm 1.03 \\
CTEQ6M1+ & 175.91 & \pm 1.02 & CTEQ6M1- & 175.89 & \pm 1.04 \\
CTEQ6M2+ & 175.92 & \pm 1.03 & CTEQ6M2- & 175.94 & \pm 1.03 \\
CTEQ6M3+ & 176.01 & \pm 1.04 & CTEQ6M3- & 175.84 & \pm 1.02 \\
CTEQ6M4+ & 175.92 & \pm 1.01 & CTEQ6M4- & 175.96 & \pm 1.02 \\
CTEQ6M5+ & 175.92 & \pm 1.00 & CTEQ6M5- & 175.94 & \pm 1.03 \\
CTEQ6M6+ & 175.88 & \pm 1.02 & CTEQ6M6- & 175.91 & \pm 1.03 \\
CTEQ6M7+ & 175.93 & \pm 1.03 & CTEQ6M7- & 175.92 & \pm 1.03 \\
CTEQ6M8+ & 176.04 & \pm 1.03 & CTEQ6M8- & 175.81 & \pm 1.02 \\
CTEQ6M9+ & 175.89 & \pm 1.01 & CTEQ6M9- & 175.98 & \pm 1.03 \\
CTEQ6M10+ & 175.92 & \pm 1.01 & CTEQ6M10- & 175.90 & \pm 1.03 \\
CTEQ6M11+ & 175.91 & \pm 1.03 & CTEQ6M11- & 175.88 & \pm 1.02 \\
CTEQ6M12+ & 175.90 & \pm 1.03 & CTEQ6M12- & 175.93 & \pm 1.03 \\
CTEQ6M13+ & 176.02 & \pm 1.04 & CTEQ6M13- & 175.76 & \pm 1.02 \\
CTEQ6M14+ & 175.91 & \pm 1.01 & CTEQ6M14- & 175.95 & \pm 1.03 \\
CTEQ6M15+ & 175.92 & \pm 1.03 & CTEQ6M15- & 175.81 & \pm 0.97 \\
CTEQ6M16+ & 175.88 & \pm 1.03 & CTEQ6M16- & 175.90 & \pm 1.04 \\
CTEQ6M17+ & 175.93 & \pm 1.04 & CTEQ6M17- & 175.90 & \pm 1.03 \\
CTEQ6M18+ & 176.02 & \pm 1.03 & CTEQ6M18- & 175.79 & \pm 1.02 \\
CTEQ6M19+ & 175.89 & \pm 1.00 & CTEQ6M19- & 175.94 & \pm 1.04 \\
CTEQ6M20+ & 175.93 & \pm 1.01 & CTEQ6M20- & 175.92 & \pm 1.03 \\
\hline
\end{tabular}

Table 51: 2 leading jets mass distribution means and uncertainty on the means from each of the 46 PDFs 


\begin{tabular}{|lrr||lcr|}
\hline PDF Set & Mass Mean & Uncertainty & PDF Set & Mass Mean & Uncertainty \\
\hline \hline CTEQ5L & 173.86 & \pm 0.74 & MRST72 & 173.83 & \pm 0.73 \\
MRST75 & 173.97 & \pm 0.71 & CTEQ6L & 173.86 & \pm 0.73 \\
CTEQ6L1 & 173.87 & \pm 0.75 & CTEQ6M & 173.92 & \pm 0.73 \\
CTEQ6M1+ & 173.87 & \pm 0.73 & CTEQ6M1- & 173.91 & \pm 0.75 \\
CTEQ6M2+ & 173.90 & \pm 0.73 & CTEQ6M2- & 173.87 & \pm 0.73 \\
CTEQ6M3+ & 173.91 & \pm 0.73 & CTEQ6M3- & 173.90 & \pm 0.72 \\
CTEQ6M4+ & 173.90 & \pm 0.74 & CTEQ6M4- & 173.92 & \pm 0.72 \\
CTEQ6M5+ & 173.88 & \pm 0.72 & CTEQ6M5- & 173.90 & \pm 0.73 \\
CTEQ6M6+ & 173.85 & \pm 0.73 & CTEQ6M6- & 173.95 & \pm 0.74 \\
CTEQ6M7+ & 173.90 & \pm 0.73 & CTEQ6M7- & 173.87 & \pm 0.73 \\
CTEQ6M8+ & 173.92 & \pm 0.73 & CTEQ6M8- & 173.87 & \pm 0.72 \\
CTEQ6M9+ & 173.88 & \pm 0.73 & CTEQ6M9- & 173.94 & \pm 0.72 \\
CTEQ6M10+ & 173.85 & \pm 0.73 & CTEQ6M10- & 173.94 & \pm 0.73 \\
CTEQ6M11+ & 173.87 & \pm 0.74 & CTEQ6M11- & 173.93 & \pm 0.74 \\
CTEQ6M12+ & 173.88 & \pm 0.73 & CTEQ6M12- & 173.87 & \pm 0.74 \\
CTEQ6M13+ & 173.92 & \pm 0.74 & CTEQ6M13- & 173.86 & \pm 0.72 \\
CTEQ6M14+ & 173.88 & \pm 0.73 & CTEQ6M14- & 173.91 & \pm 0.73 \\
CTEQ6M15+ & 173.81 & \pm 0.74 & CTEQ6M15- & 173.94 & \pm 0.68 \\
CTEQ6M16+ & 173.87 & \pm 0.74 & CTEQ6M16- & 173.91 & \pm 0.75 \\
CTEQ6M17+ & 173.88 & \pm 0.73 & CTEQ6M17- & 173.88 & \pm 0.73 \\
CTEQ6M18+ & 173.92 & \pm 0.73 & CTEQ6M18- & 173.87 & \pm 0.72 \\
CTEQ6M19+ & 173.92 & \pm 0.73 & CTEQ6M19- & 173.89 & \pm 0.73 \\
CTEQ6M20+ & 173.88 & \pm 0.73 & CTEQ6M20- & 173.91 & \pm 0.73 \\
\hline
\end{tabular}

Table 52: 2 leading jets mass distribution means and uncertainty on the means from each of the 46 PDFs 


\begin{tabular}{|c|c|c|c|c|c|}
\hline Uncert. & PDF Sets & Diff. & Uncert. & PDF Sets & Diff. \\
\hline MRST & IMRST72 - CTEQ5L & -0.07 & $\alpha_{s}$ & MRST75 - MRST72 & 0.08 \\
\hline $\mathrm{EV} 1+$ & CTEQ6M1+ - CTEQ6M & -0.10 & EV1- & CTEQ6M1- - CTEQ6M & -0.15 \\
\hline $\mathrm{EV} 2+$ & CTEQ6M2+ - CTEQ6M & -0.17 & EV2- & CTEQ6M2- - CTEQ6M & -0.05 \\
\hline EV3+ & CTEQ6M3+ - CTEQ6M & -0.04 & EV3- & CTEQ6M3- - CTEQ6M & -0.02 \\
\hline EV4+ & CTEQ6M4+ - CTEQ6M & -0.13 & EV4- & CTEQ6M4- - CTEQ6M & -0.08 \\
\hline EV5+ & CTEQ6M5+ - CTEQ6M & -0.11 & EV5- & CTEQ6M5- - CTEQ6M & -0.01 \\
\hline $\mathrm{EV} 6+$ & CTEQ6M6+ - CTEQ6M & -0.12 & EV6- & CTEQ6M6- - CTEQ6M & -0.11 \\
\hline EV7+ & CTEQ6M7+ - CTEQ6M & -0.14 & EV7- & CTEQ6M7- - CTEQ6M & -0.07 \\
\hline EV8+ & CTEQ6M8+ - CTEQ6M & -0.00 & EV8- & CTEQ6M8- - CTEQ6M & -0.04 \\
\hline EV9+ & CTEQ6M9+ - CTEQ6M & -0.13 & EV9- & CTEQ6M9- - CTEQ6M & -0.06 \\
\hline EV10+ & CTEQ6M10+ - CTEQ6M & -0.07 & EV10- & CTEQ6M10- - CTEQ6M & 0.01 \\
\hline EV11+ & CTEQ6M11+ - CTEQ6M & -0.11 & EV11- & CTEQ6M11- - CTEQ6M & -0.12 \\
\hline EV12+ & CTEQ6M12+ - CTEQ6M & -0.19 & EV12- & CTEQ6M12- - CTEQ6M & -0.09 \\
\hline EV13+ & CTEQ6M13+ - CTEQ6M & -0.03 & EV13- & CTEQ6M13- - CTEQ6M & -0.06 \\
\hline EV14+ & CTEQ6M14+ - CTEQ6M & -0.12 & EV14- & CTEQ6M14- - CTEQ6M & -0.09 \\
\hline EV15+ & CTEQ6M15+ - CTEQ6M & -0.07 & EV15- & CTEQ6M15- - CTEQ6M & 0.08 \\
\hline EV16+ & CTEQ6M16+ - CTEQ6M & -0.14 & EV16- & CTEQ6M16- - CTEQ6M & -0.15 \\
\hline EV17+ & CTEQ6M17+ - CTEQ6M & -0.16 & EV17- & CTEQ6M17- - CTEQ6M & -0.10 \\
\hline EV18+ & CTEQ6M18+ - CTEQ6M & -0.04 & EV18- & CTEQ6M18- - CTEQ6M & -0.04 \\
\hline EV19+ & CTEQ6M19+ - CTEQ6M & -0.11 & EV19- & CTEQ6M19- - CTEQ6M & -0.09 \\
\hline EV20+ & CTEQ6M20+ - CTEQ6M & -0.11 & EV20- & CTEQ6M20- - CTEQ6M & 0.00 \\
\hline \multicolumn{2}{|c|}{$\mathrm{EV}+$ Sum in Quadrature } & 0.08 & \multicolumn{2}{|c|}{ EV-Sum in Quadrature } & 0.54 \\
\hline
\end{tabular}

Table 53: Likelihood only uncertainties for the different PDFs 


\begin{tabular}{|lcr||lrr|}
\hline Uncert. & PDF Sets & Diff. & Uncert. & PDF Sets & Diff. \\
\hline \hline MRST & MRST72 - CTEQ5L & -0.04 & $\alpha_{s}$ & MRST75 - MRST72 & 0.06 \\
\hline EV1+ & CTEQ6M1+ - CTEQ6M & -0.05 & EV1- & CTEQ6M1- - CTEQ6M & -0.09 \\
EV2+ & CTEQ6M2+ - CTEQ6M & -0.02 & EV2- & CTEQ6M2- - CTEQ6M & -0.00 \\
EV3+ & CTEQ6M3+ - CTEQ6M & 0.00 & EV3- & CTEQ6M3- - CTEQ6M & 0.06 \\
EV4+ & CTEQ6M4+ - CTEQ6M & -0.10 & EV4- & CTEQ6M4- - CTEQ6M & -0.04 \\
EV5+ & CTEQ6M5+ - CTEQ6M & -0.07 & EV5- & CTEQ6M5- - CTEQ6M & -0.01 \\
EV6+ & CTEQ6M6+ - CTEQ6M & -0.06 & EV6- & CTEQ6M6- - CTEQ6M & -0.05 \\
EV7+ & CTEQ6M7+ - CTEQ6M & -0.01 & EV7- & CTEQ6M7- - CTEQ6M & -0.01 \\
EV8+ & CTEQ6M8+ - CTEQ6M & 0.03 & EV8- & CTEQ6M8- - CTEQ6M & 0.04 \\
EV9+ & CTEQ6M9+ - CTEQ6M & -0.12 & EV9- & CTEQ6M9- - CTEQ6M & -0.03 \\
EV10+ & CTEQ6M10+ - CTEQ6M & -0.06 & EV10- & CTEQ6M10- - CTEQ6M & 0.02 \\
EV11+ & CTEQ6M11+ - CTEQ6M & -0.05 & EV11- & CTEQ6M11- CTEQ6M & -0.06 \\
EV12+ & CTEQ6M12+ - CTEQ6M & -0.03 & EV12- & CTEQ6M12- - CTEQ6M & -0.03 \\
EV13+ & CTEQ6M13+ - CTEQ6M & 0.00 & EV13- & CTEQ6M13- CTEQ6M & 0.04 \\
EV14+ & CTEQ6M14+ - CTEQ6M & -0.10 & EV14- & CTEQ6M14- CTEQ6M & -0.04 \\
EV15+ & CTEQ6M15+ - CTEQ6M & -0.08 & EV15- & CTEQ6M15- CTEQ6M & 0.11 \\
EV16+ & CTEQ6M16+ - CTEQ6M & -0.06 & EV16- & CTEQ6M16- - CTEQ6M & -0.09 \\
EV17+ & CTEQ6M17+ - CTEQ6M & -0.04 & EV17- & CTEQ6M17- CTEQ6M & -0.02 \\
EV18+ & CTEQ6M18+ - CTEQ6M & 0.00 & EV18- & CTEQ6M18- CTEQ6M & 0.05 \\
EV19+ & CTEQ6M19+ - CTEQ6M & -0.12 & EV19- & CTEQ6M19- CTEQ6M & -0.05 \\
EV20+ & CTEQ6M20+ - CTEQ6M & -0.06 & EV20- & CTEQ6M20- - CTEQ6M & -0.00 \\
\hline EV+ Sum & in Quadrature & 0.15 & EV- Sum & in Quadrature & 0.30 \\
\hline
\end{tabular}

Table 54: Likelihood added uncertainties for the different PDFs 


\begin{tabular}{|c|c|c|c|c|c|}
\hline Uncert. & PDF Sets & Diff. & Uncert. & PDF Sets & Diff. \\
\hline MRST & IMRST72 - CTEQ5L & -0.21 & $\alpha_{s}$ & MRST75 - MRST72 & 0.02 \\
\hline $\mathrm{EV} 1+$ & CTEQ6M1+ - CTEQ6M & -0.00 & EV1- & CTEQ6M1- - CTEQ6M & -0.01 \\
\hline $\mathrm{EV} 2+$ & CTEQ6M2+ - CTEQ6M & 0.01 & EV2- & CTEQ6M2- - CTEQ6M & 0.03 \\
\hline EV3+ & CTEQ6M3+ - CTEQ6M & 0.10 & EV3- & CTEQ6M3- - CTEQ6M & -0.07 \\
\hline EV4+ & CTEQ6M4+ - CTEQ6M & 0.01 & EV4- & CTEQ6M4- - CTEQ6M & 0.05 \\
\hline EV5+ & CTEQ6M5+ - CTEQ6M & 0.01 & EV5- & CTEQ6M5- - CTEQ6M & 0.03 \\
\hline $\mathrm{EV} 6+$ & CTEQ6M6+ - CTEQ6M & -0.02 & EV6- & CTEQ6M6- - CTEQ6M & -0.00 \\
\hline EV7+ & CTEQ6M7+ - CTEQ6M & 0.02 & EV7- & CTEQ6M7- - CTEQ6M & 0.01 \\
\hline EV8+ & CTEQ6M8+ - CTEQ6M & 0.13 & EV8- & CTEQ6M8- - CTEQ6M & -0.10 \\
\hline EV9+ & CTEQ6M9+ - CTEQ6M & -0.02 & EV9- & CTEQ6M9- - CTEQ6M & 0.07 \\
\hline EV10+ & CTEQ6M10+ - CTEQ6M & 0.01 & EV10- & CTEQ6M10- - CTEQ6M & -0.01 \\
\hline EV11+ & CTEQ6M11+ - CTEQ6M & 0.01 & EV11- & CTEQ6M11- - CTEQ6M & -0.03 \\
\hline EV12+ & CTEQ6M12+ - CTEQ6M & -0.01 & EV12- & CTEQ6M12- - CTEQ6M & 0.02 \\
\hline EV13+ & CTEQ6M13+ - CTEQ6M & 0.11 & EV13- & CTEQ6M13- - CTEQ6M & -0.15 \\
\hline EV14+ & CTEQ6M14+ - CTEQ6M & -0.00 & EV14- & CTEQ6M14- - CTEQ6M & 0.04 \\
\hline EV15+ & CTEQ6M15+ - CTEQ6M & 0.01 & EV15- & CTEQ6M15- - CTEQ6M & -0.10 \\
\hline EV16+ & CTEQ6M16+ - CTEQ6M & -0.02 & EV16- & CTEQ6M16- - CTEQ6M & -0.01 \\
\hline EV17+ & CTEQ6M17+ - CTEQ6M & 0.02 & EV17- & CTEQ6M17- - CTEQ6M & -0.01 \\
\hline EV18+ & CTEQ6M18+ - CTEQ6M & 0.11 & EV18- & CTEQ6M18- - CTEQ6M & -0.11 \\
\hline EV19+ & CTEQ6M19+ - CTEQ6M & -0.02 & EV19- & CTEQ6M19- - CTEQ6M & 0.03 \\
\hline EV20+ & CTEQ6M20+ - CTEQ6M & 0.02 & EV20- & CTEQ6M20- - CTEQ6M & 0.01 \\
\hline \multicolumn{2}{|c|}{$\mathrm{EV}+$ Sum in Quadrature } & 0.26 & \multicolumn{2}{|c|}{ EV-Sum in Quadrature } & 0.25 \\
\hline
\end{tabular}

Table 55: 2 leading jets uncertainties for the different PDFs 


\begin{tabular}{|c|c|c|c|c|c|}
\hline Uncert. & PDF Sets & Diff. & Uncert. & PDF Sets & Diff. \\
\hline MRST & MRST72 - CTEQ5L & -0.03 & $\bar{\alpha} \alpha_{s}$ & MRST75 - MRST72 & 0.14 \\
\hline EV1+ & CTEQ6M1+ - CTEQ6M & -0.04 & EV1- & CTEQ6M1- - CTEQ6M & -0.01 \\
\hline $\mathrm{EV} 2+$ & CTEQ6M2+ - CTEQ6M & -0.02 & EV2- & CTEQ6M2- - CTEQ6M & -0.04 \\
\hline $\mathrm{EV} 3+$ & CTEQ6M3+ - CTEQ6M & -0.01 & EV3- & CTEQ6M3- - CTEQ6M & -0.02 \\
\hline EV4+ & CTEQ6M4+ - CTEQ6M & -0.01 & EV4- & CTEQ6M4- - CTEQ6M & 0.00 \\
\hline EV5+ & CTEQ6M5+ - CTEQ6M & -0.03 & EV5- & CTEQ6M5- - CTEQ6M & -0.02 \\
\hline EV6+ & CTEQ6M6+ - CTEQ6M & -0.07 & EV6- & CTEQ6M6- - CTEQ6M & 0.03 \\
\hline EV7+ & CTEQ6M7+ - CTEQ6M & -0.02 & EV7- & CTEQ6M7- - CTEQ6M & -0.05 \\
\hline $\mathrm{EV} 8+$ & CTEQ6M8+ - CTEQ6M & 0.01 & EV8- & CTEQ6M8- - CTEQ6M & -0.04 \\
\hline EV9+ & CTEQ6M9+ - CTEQ6M & -0.03 & EV9- & CTEQ6M9- - CTEQ6M & 0.02 \\
\hline EV10+ & CTEQ6M10+ - CTEQ6M & -0.06 & EV10- & CTEQ6M10- - CTEQ6M & 0.02 \\
\hline EV11+ & CTEQ6M11+ - CTEQ6M & -0.04 & EV11- & CTEQ6M11- - CTEQ6M & 0.01 \\
\hline EV12+ & CTEQ6M12+ - CTEQ6M & -0.04 & EV12- & CTEQ6M12- - CTEQ6M & -0.04 \\
\hline EV13+ & CTEQ6M13+ - CTEQ6M & 0.00 & EV13- & CTEQ6M13- - CTEQ6M & -0.06 \\
\hline EV14+ & CTEQ6M14+ - CTEQ6M & -0.03 & EV14- & CTEQ6M14- - CTEQ6M & -0.01 \\
\hline EV15+ & CTEQ6M15+ - CTEQ6M & -0.10 & EV15- & CTEQ6M15- - CTEQ6M & 0.02 \\
\hline EV16+ & CTEQ6M16+ - CTEQ6M & -0.05 & EV16- & CTEQ6M16- - CTEQ6M & -0.01 \\
\hline EV17+ & CTEQ6M17+ - CTEQ6M & -0.04 & EV17- & CTEQ6M17- - CTEQ6M & -0.03 \\
\hline EV18+ & CTEQ6M18+ - CTEQ6M & 0.00 & EV18- & CTEQ6M18- - CTEQ6M & -0.05 \\
\hline EV19+ & CTEQ6M19+ - CTEQ6M & 0.00 & EV19- & CTEQ6M19- - CTEQ6M & -0.02 \\
\hline $\mathrm{EV} 20+$ & CTEQ6M20+ - CTEQ6M & -0.03 & EV20- & CTEQ6M20- - CTEQ6M & -0.00 \\
\hline \multicolumn{2}{|c|}{$\mathrm{EV}+$ Sum in Quadrature } & 0.05 & \multicolumn{2}{|c|}{ EV-Sum in Quadrature } & 0.21 \\
\hline
\end{tabular}

Table 56: 2 leading jets added uncertainties for the different PDFs 
The systematic error due to the PDFs are:

$$
\begin{aligned}
\sigma_{P D F}^{s y s}(L) & ={ }_{-0.55}^{+0.11} \mathrm{GeV} & \sigma_{P D F}^{\text {sys }}(L+) & ={ }_{-0.31}^{+0.16} \mathrm{GeV} \\
\sigma_{P D F}^{\text {sys }}(2 L J) & ={ }_{-0.25}^{+0.26} \mathrm{GeV} & \sigma_{P D F}^{\text {sys }}(2 L J+) & ={ }_{-0.25}^{+0.15} \mathrm{GeV}
\end{aligned}
$$

\subsubsection{ISR/FSR}

\begin{tabular}{lcccc}
\hline MC Sample & Likelihood & Added & 2LJ & 2LJ Added \\
\hline \hline ttop75 & $174.75 \pm 0.48$ & $175.08 \pm 0.42$ & $174.48 \pm 0.48$ & $175.10 \pm 0.41$ \\
otop03 & $175.21 \pm 0.45$ & $174.66 \pm 0.40$ & $175.86 \pm 0.45$ & $175.26 \pm 0.40$ \\
otop04 & $175.03 \pm 0.36$ & $175.28 \pm 0.32$ & $175.08 \pm 0.36$ & $175.30 \pm 0.31$ \\
\hline
\end{tabular}

Table 57: Mass distribution means and the uncertainty on the means for the IFS/FSR systematic samples.

\begin{tabular}{lcccc}
\hline MC Sample & Likelihood & Added & 2LJ & 2LJ Added \\
\hline \hline otopo3 & $0.46 \pm 0.66$ & $-0.42 \pm 0.58$ & $1.38 \pm 0.66$ & $0.16 \pm 0.57$ \\
otopo4 & $0.28 \pm 0.60$ & $0.20 \pm 0.53$ & $0.60 \pm 0.60$ & $0.20 \pm 0.52$ \\
\hline
\end{tabular}

Table 58: Difference of the ISR/FSR systematic samples from the nominal sample.

The systematic errors due to Initial and Final State Radiation are:

$$
\begin{aligned}
& \sigma_{I F S R}^{\text {sys }}(L)= \pm 0.48 G e V \quad \sigma_{I F S R}^{\text {sys }}(L+)= \pm 0.42 G e V \\
& \sigma_{I F S R}^{\text {sys }}(2 L J)= \pm 1.38 G e V \quad \sigma_{I F S R}^{\text {sys }}(2 L J+)= \pm 0.41 G e V
\end{aligned}
$$




\subsubsection{Generator}

\begin{tabular}{lcccc}
\hline MC Sample & Likelihood & Added & 2LJ & 2LJ Added \\
\hline \hline ttop75 & $174.75 \pm 0.48$ & $175.08 \pm 0.42$ & $174.48 \pm 0.48$ & $175.10 \pm 0.41$ \\
otop1s & $175.10 \pm 0.54$ & $175.11 \pm 0.46$ & $174.78 \pm 0.54$ & $174.60 \pm 0.46$ \\
\hline \hline Shift & $-0.35 \pm 0.72$ & $-0.04 \pm 0.62$ & $-0.30 \pm 0.72$ & $0.51 \pm 0.62$ \\
\hline
\end{tabular}

Table 59: Mass distribution means and the uncertainty on the means for the PYTHIA and HERWIG samples as well as the difference of the HERWIG samples from the nominal (PYTHIA) sample.

The systematic errors due to different MC Generators are:

$$
\begin{aligned}
& \sigma_{M C}^{s y s}(L)= \pm 0.48 G e V \quad \sigma_{M C}^{s y s}(L+)= \pm 0.42 G e V \\
& \sigma_{M C}^{s y s}(2 L J)= \pm 0.48 G e V \quad \sigma_{M C}^{s y s}(2 L J+)= \pm 0.51 G e V
\end{aligned}
$$

\subsubsection{Background Fraction}

\begin{tabular}{l|c|c|r}
\hline Background & Mass Mean & Mass Mean & Diff/2 \\
\hline \hline Fakes & 174.99 & 174.99 & -0.00 \\
WW & 175.00 & 175.00 & -0.00 \\
WZ & 175.00 & 175.00 & -0.00 \\
$\mathrm{ZZ}$ & 175.00 & 175.00 & -0.00 \\
$\mathrm{~W} \gamma$ & 175.00 & 175.00 & -0.00 \\
$\mathrm{Z} \rightarrow$ ee & 174.99 & 175.00 & -0.01 \\
$\mathrm{Z} \rightarrow \mu \mu$ & 174.56 & 174.59 & -0.02 \\
$\mathrm{Z} \rightarrow \tau \tau$ & 175.03 & 174.98 & 0.03 \\
All & 175.01 & 175.00 & 0.00 \\
\hline Quad Sum & Shifted Up & Shifted Down & 0.03 \\
\hline Nominal & \multicolumn{3}{|c}{$174.75 \pm 0.48$} \\
\hline
\end{tabular}

Table 60: Likelihood only mass distribution means and the systematic uncertainties for each of the shifted backgrounds and the sum in quadrature of the systematic uncertainties. 


\begin{tabular}{l|c|c|c}
\hline Background & Mass Mean & Mass Mean & Diff/2 \\
\hline \hline Fakes & 175.12 & 175.15 & -0.01 \\
WW & 175.14 & 175.15 & -0.00 \\
WZ & 175.14 & 175.15 & -0.00 \\
ZZ & 175.14 & 175.15 & -0.00 \\
W & 175.14 & 175.15 & -0.00 \\
$\mathrm{Z} \rightarrow$ ee & 175.14 & 175.15 & -0.00 \\
$\mathrm{Z} \rightarrow \mu \mu$ & 174.33 & 174.35 & -0.01 \\
$\mathrm{Z} \rightarrow \tau \tau$ & 175.19 & 175.10 & 0.04 \\
All & 175.13 & 175.15 & -0.01 \\
\hline Quad Sum & Shifted Up & Shifted Down & 0.05 \\
\hline Nominal & \multicolumn{3}{|c}{$175.08 \pm 0.42$} \\
\hline
\end{tabular}

Table 61: Likelihood added mass distribution means and the systematic uncertainties for each of the shifted backgrounds and the sum in quadrature of the systematic uncertainties.

\begin{tabular}{l|c|c|r}
\hline Background & Mass Mean & Mass Mean & Diff/2 \\
\hline \hline Fakes & 176.07 & 176.01 & 0.03 \\
WW & 176.03 & 176.06 & -0.01 \\
WZ & 176.03 & 176.06 & -0.01 \\
$\mathrm{ZZ}$ & 176.03 & 176.06 & -0.01 \\
$\mathrm{~W} \gamma$ & 176.03 & 176.06 & -0.01 \\
$\mathrm{Z} \rightarrow$ ee & 176.03 & 176.06 & -0.01 \\
$\mathrm{Z} \rightarrow \mu \mu$ & 176.05 & 176.01 & 0.02 \\
$\mathrm{Z} \rightarrow \tau \tau$ & 176.13 & 175.99 & 0.07 \\
All & 176.05 & 176.07 & -0.01 \\
\hline Quad Sum & Shifted Up & Shifted Down & 0.08 \\
\hline Nominal & \multicolumn{3}{|c}{$174.48 \pm 0.48$} \\
\hline \multicolumn{4}{|c}{}
\end{tabular}

Table 62: 2 leading jets mass distribution means and the systematic uncertainties for each of the shifted backgrounds and the sum in quadrature of the systematic uncertainties. 


\begin{tabular}{l|c|c|r}
\hline Background & Mass Mean & Mass Mean & Diff/2 \\
\hline \hline Fakes & 173.86 & 173.81 & 0.03 \\
$W W$ & 173.86 & 173.88 & -0.01 \\
WZ & 173.86 & 173.88 & -0.01 \\
ZZ & 173.86 & 173.88 & -0.01 \\
W & 173.86 & 173.88 & -0.01 \\
$\mathrm{Z} \rightarrow$ ee & 173.86 & 173.88 & -0.01 \\
$\mathrm{Z} \rightarrow \mu \mu$ & 173.20 & 173.21 & -0.00 \\
$\mathrm{Z} \rightarrow \tau \tau$ & 173.92 & 173.79 & 0.07 \\
All & 173.82 & 173.85 & -0.02 \\
\hline Quad Sum & Shifted Up & Shifted Down & 0.07 \\
\hline Nominal & \multicolumn{3}{|c}{$175.10 \pm 0.41$} \\
\hline
\end{tabular}

Table 63: 2 leading jets mass distribution means and the systematic uncertainties for each of the shifted backgrounds and the sum in quadrature of the systematic uncertainties.

The systematic errors due to different background fractions are:

$$
\begin{aligned}
\sigma_{B G}^{\text {sys }}(L) & = \pm 0.48 G e V & \sigma_{B G}^{\text {sys }}(L+) & = \pm 0.42 G e V \\
\sigma_{B G}^{\text {sys }}(2 L J) & = \pm 0.48 G e V & \sigma_{B G}^{\text {sys }}(2 L J+) & = \pm 0.41 G e V
\end{aligned}
$$

\subsection{6 b-Jet Energy Scale}

\begin{tabular}{l|c|c|r}
\hline Systematic & Mass Mean & Mass Mean & Syst. \\
\hline \hline b-JES & $176.62 \pm 0.53$ & $173.63 \pm 0.54$ & 0.30 \\
Branching Ratio & $175.00 \pm 0.76$ & $174.99 \pm 0.77$ & 0.01 \\
Fragmentation & $174.96 \pm 0.76$ & $174.96 \pm 0.77$ & -0.01 \\
\hline Quad Sum & Shifted Up (ADO) & Shifted Down (SLD) & 0.30 \\
\hline Nominal & \multicolumn{2}{|c}{$174.75 \pm 0.48$} \\
\hline
\end{tabular}

Table 64: Likelihood only mass distribution means and the uncertainty on the means for the $b$-Jet systematic studies. 


\begin{tabular}{l|c|c|c}
\hline Systematic & Mass Mean & Mass Mean & Syst. \\
\hline \hline b-JES & $176.78 \pm 0.47$ & $174.17 \pm 0.48$ & 0.26 \\
Branching Ratio & $175.15 \pm 0.68$ & $175.13 \pm 0.68$ & 0.02 \\
Fragmentation & $175.10 \pm 0.68$ & $175.12 \pm 0.68$ & -0.02 \\
\hline Quad Sum & Shifted Up (ADO) & Shifted Down (SLD) & 0.26 \\
\hline Nominal & \multicolumn{2}{|c}{$175.08 \pm 0.42$} \\
\hline
\end{tabular}

Table 65: Likelihood added mass distribution means and the uncertainty on the means for the $b$-Jet systematic studies.

\begin{tabular}{l|c|c|c}
\hline Systematic & Mass Mean & Mass Mean & Syst. \\
\hline \hline$b$-JES & $176.81 \pm 0.54$ & $173.68 \pm 0.55$ & 0.31 \\
Branching Ratio & $176.07 \pm 1.05$ & $176.03 \pm 1.04$ & 0.04 \\
Fragmentation & $176.08 \pm 1.04$ & $176.05 \pm 1.05$ & 0.03 \\
\hline Quad Sum & Shifted Up (ADO) & Shifted Down (SLD) & 0.32 \\
\hline Nominal & \multicolumn{2}{|c}{$174.48 \pm 0.48$} \\
\hline
\end{tabular}

Table 66: 2 leading jets mass distribution means and the uncertainty on the means for the $b$-Jet systematic studies.

\begin{tabular}{l|c|c|r}
\hline Systematic & Mass Mean & Mass Mean & Syst. \\
\hline \hline b-JES & $176.35 \pm 0.47$ & $173.89 \pm 0.48$ & 0.25 \\
Branching Ratio & $173.86 \pm 0.74$ & $173.87 \pm 0.74$ & -0.01 \\
Fragmentation & $173.85 \pm 0.74$ & $173.87 \pm 0.74$ & -0.02 \\
\hline Quad Sum & Shifted Up (ADO) & Shifted Down (SLD) & 0.25 \\
\hline Nominal & \multicolumn{2}{|c}{$175.10 \pm 0.41$} \\
\hline
\end{tabular}

Table 67: 2 leading jets added mass distribution means and the uncertainty on the means for the $b$-Jet systematic studies. 
The systematic errors due to the b-Jet Energy Scale are:

$$
\begin{aligned}
\sigma_{b J e s}^{\text {sys }}(L) & = \pm 0.48 G e V & \sigma_{b J e s}^{\text {sys }}(L+) & = \pm 0.42 G e V \\
\sigma_{b J e s}^{\text {sys }}(2 L J) & = \pm 0.48 G e V & \sigma_{b J e s}^{\text {sys }}(2 L J+) & = \pm 0.41 G e V
\end{aligned}
$$

\subsubsection{Lepton $P_{T}$}

\begin{tabular}{l|c|c|c|c}
\hline MC Sample & Likelihood & Added & 2LJ & 2LJ Added \\
\hline \hline Nominal & $174.75 \pm 0.48$ & $175.08 \pm 0.42$ & $174.48 \pm 0.48$ & $175.10 \pm 0.41$ \\
Shifted Down & $174.74 \pm 0.62$ & $175.31 \pm 0.55$ & $174.50 \pm 0.62$ & $174.75 \pm 0.55$ \\
Shifted Up & $175.23 \pm 0.54$ & $175.39 \pm 0.47$ & $175.17 \pm 0.54$ & $174.95 \pm 0.47$ \\
\hline Systematic Shift & 0.25 & 0.15 & 0.35 & -0.17 \\
\hline
\end{tabular}

Table 68: Mass distribution means and the uncertainty on the means for the shifted lepton energy systematic study.

The systematic errors due to Lepton $P_{T}$ are:

$$
\begin{aligned}
\sigma_{L P T}^{s y s}(L) & = \pm 0.48 G e V & \sigma_{L P T}^{s y s}(L+) & = \pm 0.42 G e V \\
\sigma_{L P T}^{s y s}(2 L J) & = \pm 0.48 G e V & \sigma_{L P T}^{s y s}(2 L J+) & = \pm 0.41 G e V
\end{aligned}
$$

\subsubsection{Pile-Up}

$$
\begin{aligned}
\sigma_{P U_{K n o w n}}^{\text {sys }} & = \pm 0.14 \mathrm{GeV} \\
\sigma_{P U_{U n k n o w n}}^{\text {sys }}(L) & = \pm 1.22 \mathrm{GeV} \\
\sigma_{P U_{U \text { Unnown }}}^{\text {sys }}(L+) & = \pm 0.72 \mathrm{GeV} \\
\sigma_{P U_{\text {Unknown }}}^{\text {sys }}(2 \mathrm{LJ}) & = \pm 1.32 \mathrm{GeV} \\
\sigma_{P U_{U n k n o w n}}^{\text {sys }}(2 L J+) & = \pm 0.90 \mathrm{GeV}
\end{aligned}
$$

The systematic errors due to Pile-up are: 


\begin{tabular}{c|cccc}
\hline Variable & Likelihood & L Added & 2LJ & 2LJ Added \\
\hline \hline$<N I N T>($ data $)$ & 1.90 & 1.90 & 1.90 & 1.90 \\
$<N I N T>(M C)$ & 1.49 & 1.49 & 1.39 & 1.39 \\
$m_{P U}$ & 0.35 & & & \\
$\sigma_{m_{P U}}$ & 0.54 & & & \\
$\sigma_{L 4}^{\text {sys }}$ & -0.29 & -0.17 & -0.25 & -0.17 \\
\hline
\end{tabular}

Table 69: The values of the various variables that go into calculating the systematic uncertainty due to pile-up.

$$
\begin{aligned}
& \sigma_{P U}^{s y s}(L)= \pm 1.22 G e V \quad \sigma_{P U}^{s y s}(L+)= \pm 0.72 G e V \\
& \sigma_{P U}^{\text {sys }}(2 L J)= \pm 1.32 G e V \quad \sigma_{P U}^{\text {sys }}(2 L J+)= \pm 0.90 G e V
\end{aligned}
$$

\subsubsection{Color Reconnection}

\begin{tabular}{lcccc}
\hline MC Sample & Likelihood & Added & 2LJ & 2LJ Added \\
\hline \hline ctopsd & $171.54 \pm 0.93$ & $172.40 \pm 0.85$ & $171.45 \pm 0.95$ & $172.29 \pm 0.85$ \\
ctopse & $173.06 \pm 0.92$ & $172.13 \pm 0.85$ & $172.84 \pm 0.93$ & $171.77 \pm 0.84$ \\
\hline \hline Shift & $-1.52 \pm 1.31$ & $0.27 \pm 1.20$ & $-1.39 \pm 1.33$ & $0.52 \pm 1.20$ \\
\hline
\end{tabular}

Table 70: Mass distribution means and the uncertainty on the means for the two color reconnection samples as well as the difference.

The systematic errors due to Color Reconnection are:

$$
\begin{aligned}
\sigma_{C R}^{\text {sys }}(L) & = \pm 1.52 \mathrm{GeV} \quad \sigma_{C R}^{\text {sys }}(L+)= \pm 0.85 \mathrm{GeV} \\
\sigma_{C R}^{\text {sys }}(2 L J) & = \pm 1.39 \mathrm{GeV} \quad \sigma_{C R}^{\text {sys }}(2 L J+)= \pm 0.85 \mathrm{GeV}
\end{aligned}
$$

\subsubsection{Systematic Summary}

The Total Systematic Uncertainties from all sources are 


$$
\begin{aligned}
& \sigma_{T O T}^{\text {sys }}(L)= \pm 2.25 G e V \quad \sigma_{T O T}^{\text {sys }}(L+)= \pm 2.04 G e V \\
& \sigma_{T O T}^{\text {sys }}(2 L J)= \pm 2.42 G e V \quad \sigma_{T O T}^{\text {sys }}(2 L J+)= \pm 1.92 G e V
\end{aligned}
$$

\begin{tabular}{lcccc}
\hline Source & Likelihood & Likelihood + & 2LJ & 2LJ+ \\
\hline \hline Jet Energy Scale & $\pm 0.97 \mathrm{GeV}$ & $\pm 0.97 \mathrm{GeV}$ & $\pm 0.98 \mathrm{GeV}$ & $\pm 0.88 \mathrm{GeV}$ \\
PDF & $\pm 0.55 \mathrm{GeV}$ & $\pm 0.31 \mathrm{GeV}$ & $\pm 0.26 \mathrm{GeV}$ & $\pm 0.25 \mathrm{GeV}$ \\
ISR/FSR & $\pm 0.48 \mathrm{GeV}$ & $\pm 0.42 \mathrm{GeV}$ & $\pm 1.38 \mathrm{GeV}$ & $\pm 0.41 \mathrm{GeV}$ \\
MC Generator & $\pm 0.48 \mathrm{GeV}$ & $\pm 0.42 \mathrm{GeV}$ & $\pm 0.48 \mathrm{GeV}$ & $\pm 0.51 \mathrm{GeV}$ \\
Background & $\pm 0.48 \mathrm{GeV}$ & $\pm 0.42 \mathrm{GeV}$ & $\pm 0.48 \mathrm{GeV}$ & $\pm 0.41 \mathrm{GeV}$ \\
b-Jet Energy Scale & $\pm 0.48 \mathrm{GeV}$ & $\pm 0.42 \mathrm{GeV}$ & $\pm 0.48 \mathrm{GeV}$ & $\pm 0.41 \mathrm{GeV}$ \\
Lepton P & $\pm 0.48 \mathrm{GeV}$ & $\pm 0.42 \mathrm{GeV}$ & $\pm 0.48 \mathrm{GeV}$ & $\pm 0.41 \mathrm{GeV}$ \\
Pile-up & $\pm 1.22 \mathrm{GeV}$ & $\pm 0.72 \mathrm{GeV}$ & $\pm 1.32 \mathrm{GeV}$ & $\pm 0.90 \mathrm{GeV}$ \\
Color Reconnection & $\pm 1.52 \mathrm{GeV}$ & $\pm 0.85 \mathrm{GeV}$ & $\pm 1.39 \mathrm{GeV}$ & $\pm 0.85 \mathrm{GeV}$ \\
\hline \hline Total Systematic Uncertainty & $\pm 2.49 \mathrm{GeV}$ & $\pm 1.78 \mathrm{GeV}$ & $\pm 2.74 \mathrm{GeV}$ & $\pm 1.82 \mathrm{GeV}$ \\
\hline
\end{tabular}

Table 71: Systematic errors from various sources 


\section{Chapter 8 - Results}

\subsection{Introduction}

In this final chapter, results will be presented from this analysis. Also, there will be a comparison of the results of this method to results obtained by other methods in the di-lepton, lepton+jets, and all hadronic channels from both the CDF and DØ experiments.

\subsection{Results}

The Dalitz-Goldstein top mass fitting technique has been applied to the di-lepton events in Run II of the CDF experiment and the top quark mass has been determined to be:

$$
\begin{aligned}
& M_{\text {top }}=172.3 \pm 3.4(\text { stat. }) \pm 2.0(\text { syst. }) \mathrm{GeV} / \mathrm{c}^{2} . \\
& \left.M_{\text {top }}=170.5 \pm 3.7 \text { (stat. }\right) \pm 1.8 \text { (syst.) } \mathrm{GeV} / \mathrm{c}^{2} .
\end{aligned}
$$

Table 72 shows the uncorrected (raw) results for each of the four ways of choosing a combination from an event with and without b-tagging. Using Eqs. 61 through 64 and 93 through 96, the raw masses can be corrected. To correct the raw RMSs, Eq. 65 and the values from Tables 15 and 44 where used. Table 73 shows the fully corrected results.

\begin{tabular}{l|ll}
\hline Method & Mass & Statistical Error \\
\hline \multicolumn{3}{c}{ without } \\
-tagging \\
\hline Likelihood Only & 163.6 & \pm 0.76 \\
Likelihood Added & 163.3 & \pm 0.72 \\
2 Leading Jets & 162.7 & \pm 1.25 \\
2 Leading Jets Added & 162.8 & \pm 1.04 \\
\hline \multicolumn{3}{c}{ with b-tagging } \\
\hline Likelihood Only & 164.4 & \pm 1.28 \\
Likelihood Added & 163.0 & \pm 1.23 \\
2 Leading Jets & 164.4 & \pm 1.24 \\
2 Leading Jets Added & 162.9 & \pm 1.20 \\
\hline
\end{tabular}

Table 72: Results without any corrections. (Raw) 


\begin{tabular}{l|cccc}
\hline Method & Mass & Statistical (expected SE) & Systematic & Errors in Quad. \\
\hline \multicolumn{5}{c}{ without b-tagging } \\
\hline Likelihood Only & 170.7 & $\pm 4.20(5.25)$ & \pm 2.25 & \pm 4.76 \\
Likelihood Added & 172.3 & $\pm 3.41(4.87)$ & \pm 2.04 & \pm 3.97 \\
2 Leading Jets & 168.8 & $\pm 6.88(5.28)$ & \pm 2.42 & \pm 7.29 \\
2 Leading Jets Added & 170.3 & $\pm 5.00(4.98)$ & \pm 1.92 & \pm 5.36 \\
\hline \multicolumn{5}{c}{ with b-tagging } \\
\hline Likelihood Only & 170.7 & $\pm 4.73(5.46)$ & \pm 2.49 & \pm 5.34 \\
Likelihood Added & 170.5 & $\pm 3.74(4.75)$ & \pm 1.78 & \pm 4.14 \\
2 Leading Jets & 170.1 & $\pm 4.50(5.29)$ & \pm 2.74 & \pm 5.27 \\
2 Leading Jets Added & 169.7 & $\pm 3.54(4.56)$ & \pm 1.82 & \pm 3.98 \\
\hline
\end{tabular}

Table 73: Final results with corrections.

The results shown in Eqs. 122 and 123 have been chosen to be the the main results from this analysis based on the facts that they use all jets in an event and consistently have low systematic errors. They are therefore considered to be the best measurements from this method.

\subsection{Comparison of Results}

Figure 50 shows all of the results from this analysis and compares them to the combined average from the Tevatron. Figure 51 shows various top quark mass measurements from both CDF and DØ. Highlighted in dark gray is the result from this analysis. The Dalitz-Goldstein results are consistent with all previous measurements and the combined average. 
Mass of the Top Quark

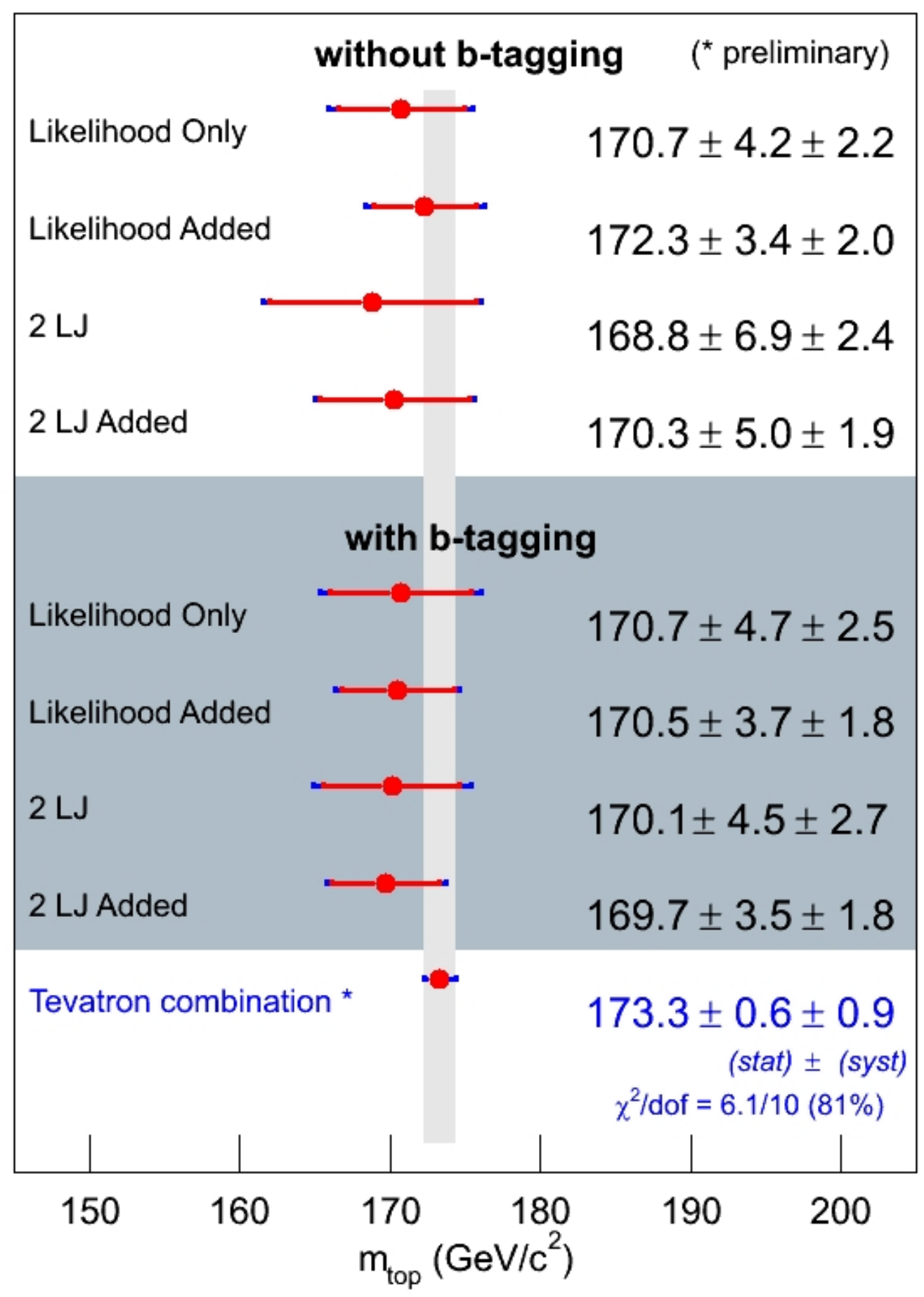

Figure 50: Top quark mass measurements from the Dalitz-Goldstein method. The red error bars represent the statistical error while the blue error bars represent the statistical and systematic errors added in quadrature. The vertical gray bar shows the interval covered by the combined top quark mass value and errors from all previous measurements at the Tevatron. 
Mass of the Top Quark

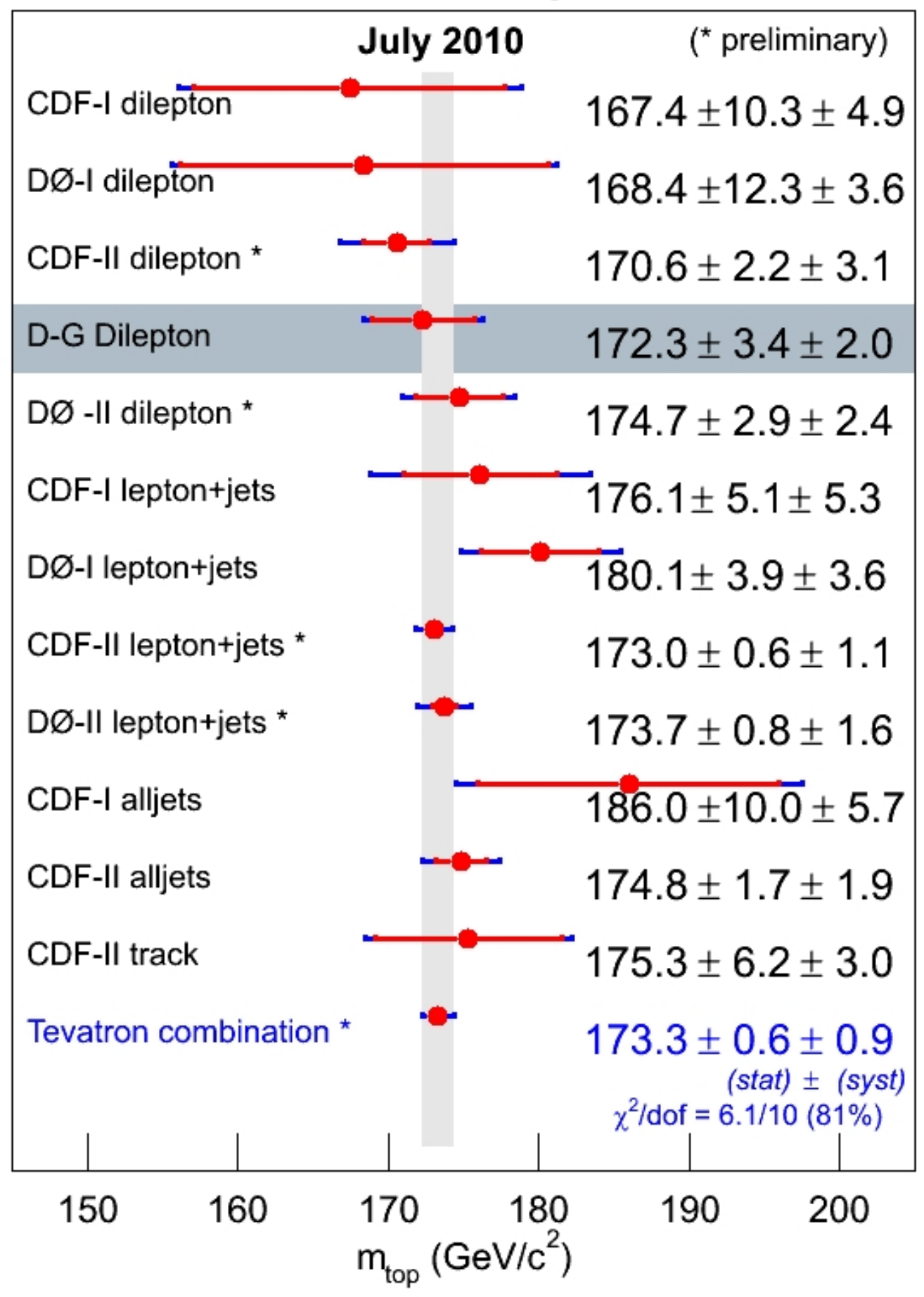

Figure 51: Top quark mass measurements from the Tevatron. The red error bars represent the statistical error while the blue error bars represent the statistical and systematic errors added in quadrature. The vertical gray bar shows the interval covered by the combined top quark mass value and errors from all previous measurements at the Tevatron. 


\section{A Error on the Mean and Pull of Ensembles of Pseudo- Experiments - The Bootstrap Technique}

A detailed explanation of this technique can be found in CDF note 9081[19]. When calculating the error on a variable from an ensemble of PEs, the usual statistics of calculating an error related to the $\sqrt{N}$, where $N$ is the number of PEs, does not apply. The problem that occurs is that as $N$ increases, the error on something like the mean will decrease, and can be effectively made to vanish; however, no new information is added as $N$ increases. Clearly, this is not correct.

In order to overcome this problem, the "Bootstrap Technique" was employed. The technique begins with a parent distribution of $n$ events. From the distribution, $C$ child distributions are created by randomly sampling $n$ events with replacement from the parent distribution. Each of the $C$ child distributions will have the same characteristics as the parent, such as the mean and width; however, each will be unique in its composition of events. With each of the $C$ child distributions, a set of PEs is created which will yield such variables as a mass mean and pull. In the end, there will be $C$ of each of these variable. For each variable, a histogram is filled. The error on a particular variable will be the width of this final histogram divided by the $\sqrt{C}$. In this analysis, $C$ was set to 50 . 


\section{B Methods for Calculating Systematic Uncertainties}

When calculating the systematic uncertainties for this analysis, the methods prescribed by the CDF Top Mass Group[20] [17] were used. Below is a copy of those prescriptions.

(0) Interpretation of shifts

- The interpretation of all systematics with +/- 1 sigma shifts should now go as follows. The systematic is half the difference between positive and negative mass results, unless both positive and negative go in the same direction from nominal, in which case the systematic is half the largest difference from nominal. This change applies to: IFSR, the semi-leptonic decay component of bJES, and lepton pt systematics. (JES and PDF systematics were already using the half-difference approach.)

(1/2) Jet Energy Scale / Residual JES

- Use tag jetCorr12, which is valid for all runII data up to p13, or newer, as per the recommendations of the jet corrections group

- Evaluate the separate uncertainties for ALL levels of the JES systematic (including higher levels in case you only correct up to L5). Add those pieces in quadrature to get the systematic on the top mass.

- The "total JES systematic" (all levels at once) can be used as a cross-check; this crosscheck is in fact recommended for analyses without in situ calibration.

- If your background is evaluated from MC, apply the same +- one sigma JES variations to your dominant background sources simultaneously with the signal variations

- You should rerun event selection to pick up events moving in and out of your sample with the shifted jet energies. So you will have a different number of events in e.g. the L5+ sample to use as pseudo-data than you do in the nominal sample. However, you do not need to change the number of events of each type you draw for a given pseudo-experiment.

(3) PDF

- Some information about PDFs: CTEQ6, hep-ph/0201195, hep-ph/0303013. 
- For gen6 analyses, please follow: Joint Physics Instructions

- Some initial information, go to: Joint Physics from April 2, 2004

- Add in quadrature the difference between CTEQ6M 20 pairs of eigenvectors

- Add in quadrature the difference between MRST with two different values of $\Lambda$ QCD (e.g. $\operatorname{MRST} 72 \Lambda \mathrm{QCD}=228 \mathrm{MeV}$ vs. $\mathrm{MRST} 75 \Lambda \mathrm{QCD}=300 \mathrm{MeV})$

- Check CTEQ5L and MRST central values are consistent

- Add in quadrature the effect of reweighing gg contribution from $5 \%$ (LO) to $20 \%(=15+5)$ $(\mathrm{NLO})$

Note: Updated recipe for PDF uncertainties (April 30, 2008)

- for "a" $\equiv$ quadrature sum of CTEQ eigenvectors and "b" $\equiv$ difference between MRST72 and CTEQ5L

- Old recipe: If $\mathrm{b}>\mathrm{a}$, add $\mathrm{b}$ and $\mathrm{a}$ in quadrature otherwise just take a.

- Modified recipe: simply taking the larger of a or b

- In all cases you still need to additionally add in quadrature the differences due to other effects: changes in $a_{l p h}$ by comparing MRST72 and MRST75, effect of reweighing gg contribution.

(4) ISR/FSR

- Use ISR/FSR less/more ttbar samples. Use the new IFSR less/more ttbar samples: otop03 and otop04. (See CDF 6804 for some documentation on the general idea behind these samples.) In the new samples, ISR and FSR are varied simultaneously.

- Find the largest difference between the nominal sample and otop03 or otop04. Calculate the uncertainty on that difference due to sample statistics (only the part due to signal stats, since you're using the same background events in both sets of PEs!). Use the larger of the largest difference or its uncertainty as your systematic. 
- For more info see

http://www-cdf.fnal.gov/internal/physics/top/run2mass/ifsr_moreinfo.html.

(5) MC Generator

- For the generator systematic, we quote the difference between the mass values obtained in PEs with Herwig and Pythia Monte Carlo generators. Other generators are also under investigation and should be analyzed as cross-checks.

- As for HERWIG:

- The old htop75 sample had incorrect JIMMY settings.

- The sample otop01 should be the same as otop1s, but otop01 has $0.5 \mathrm{M}$ events, while otop1s has 4M. We require to use otop1s for HERWIG.

- As for ALPGEN:

- For the time being there are three ALPGEN samples: otop1q which is ttbar inclusive and atop0p+atop $1 \mathrm{p}$ which are ALPGEN $t \mathrm{t}+0 \mathrm{p}$ and $\mathrm{tt}+1 \mathrm{p}$ respectively. The difference between these two ALPGEN models probes the importance of the higher-order ME.

- ALPGEN samples of $\mathrm{tt}+0 \mathrm{p}$ and $\mathrm{tt}+1 \mathrm{p}$ should be combined according to the crosssections listed on the the top group MC page.

- It seems as though our ALPGEN samples have been generated with zero (or very small) top width. Besides being plain wrong, this causes a bias in the ALPGEN samples. The top quark Breit-Wigner interacts with the PDFs such that a significantly lower width results in a larger generated top mass on average.

- Because of the problem with the top width, ALPGEN is not part of the generator systematic for $2 \mathrm{fb}-1$. No need to run on these samples until the problem is resolved.

- As for MC@NLO:

- For the blessing, we require to cross-check your result with MC@NLO(mtop75, ctop75) samples. 
- Note that MC@NLO samples have 10\% of events with negative weight. This should be taken into account when running PEs!

* The weight is not directly stored in TopNtuple. Instead, as a workaround, it is encoded in the hepg energy of the proton vs anti-proton.

* In practice: hepg. $\mathrm{E}[0]=980$ for $\mathrm{W}=+1$ and hepg. $\mathrm{E}[0]=980.09997$ for $\mathrm{W}=-1$.

- mtop75 and ctop75 differ in the PDFs used. See the top group MC page for details.

- Finally, there are random number issues with these MC@NLO samples. Events are duplicated many times within the sample at the hard-scatter level (though the showering/hadronization is then not identical). So the statistical uncertainties on quantities derived from these samples are not understood!

(6) Background fraction and shape

- This will depend on your channel and analysis. Please take into account how your top mass measurement is affected by the background fraction and the modeling of the shape

- We suggest to compare the different background shapes for different Q2 values using ALPGEN MC samples (4MW2, MW2/4). These are currently available at least for $\mathrm{W}(\mathrm{e}, \mathrm{mu})+\mathrm{bb}+\mathrm{np}:$ btop $[\mathrm{e}-\mathrm{p}] \mathrm{w}$.

(7) b-tagging efficiency

- This systematic has been removed because it is typically vanishingly small. However, analyses that might be particularly sensitive to an Et-dependent mismodeling of the $b$ tagging efficiency (e.g. those using L2D as an observable!) should still evaluate a related systematic.

(8) $b$-jet energy scale

Take into account the $b$-jet energy scale uncertainty. This systematic comes from three main components:

- semi-leptonic branching ratios - move b and c BRs together by +-1 sigma

- fragmentation - reweight to LEP/SLD Bowler parameters 
- To compute the two above effects, Erik and Jahred prepared a code that can be found at the following

http://www-cdf.fnal.gov/internal/physics/top/run2mass/BSpackage.html

- calorimeter response effect:

- run $\mathrm{S}+\mathrm{B}$ pseudo-experiments where the $b$-jets have their input energies shifted by $+/-1 \%$

- the $b$-jets are identified using hepg information; other groups only identified signal $b$-jets (ie. ignored real Bjets in Bgd)

- the energies are shifted so that their inputs to the kinematic fit (or whatever the equivalent is for a given analysis method) are $+/-1 \%$ higher/lower than when using the default jet energy corrections

- in these studies we've ignored changed in the acceptance on account of these shifts and ignored changes in the ratio of single-to-double tag events (Btag efficiency doesn't have that steep of a dependence on Bjet energy that $1 \%$ shifts should dramatically change any of this relative to dflt)

- compare the mean fitted Mtop for these two shifted samples with the mean fitted Mtop using default jet energy corrections; from this you can estimate that a $1 \%$ shift in Bjet energy corresponds to a X.X GeV shift in top mass

- your final systematic needs to be scaled to $0.2 \%$, which is our best estimate of how 1sigma changes to calorimeter response affect the $b$-jet energy scale. Thus this specific systematic will be $0.2^{*} \mathrm{X} . \mathrm{X}=\mathrm{Y}$.Y GeV

(9) Lepton PT

- A $1 \%$ uncertainty on the lepton energy scale needs to translated into Mtop and added in quadrature to the other uncertatinties. The scale should be shifted separately for electrons and muons, i.e. those are uncorrelated systematic effects.

(10) Pile-up 
- There is a change in terminology here: for public presentation instead of "multiple interaction" use "pileup": from Lina Galtieri's e-mail:"in talking to Seymour, Torbjorn Sjostrand and others, I realized that our systematics from Multiple Interactions is taken to mean systematics from PARTON MULTIPLE INTERACTION, not from MULTIPLE P-P COLLISIONS". Note: At LHC pileup can also include information from more then one event due to slow recording of event information. But that's not the case at Tevatron.

- To estimate the pileup systematic there are two pieces.

- Known mismodeling

* The first piece is related to a known midmodeling: our Monte Carlo does not have the same luminosity profile as the data we compare to it. We use MC datasets with a luminosity profile corresponding to just the first $1.2 \mathrm{fb}-1$ of data. Additionally, the conversion from luminosity to number of minbias events was wrong by $20 \%$ in datasets of that vintage.

* Calculate the importance of this mismodeling on one sample (presumably 175 GeV). Possible approaches:

- Run PEs using reweighting, where the weights depend on the number of interactions in the event, and the reweighting reproduces the Nint profile of the data. Find the difference (and its uncertainty) between these PEs and the nominal PEs.

- Run batches of PEs where the input events are segregated by number of interactions. Plot the average fitted mass vs Nint. Fit a pol1. Hopefully the slope is within say two sigma of zero. Multiply the slope, or its uncertainty, by the difference between the average number of interactions in data and MC.

- Something else convincing.

- Unknown mismodeling

* The second piece is related to possible mismodeling of the minbias events, which would induce a systematic shift even if the correct luminosity profile were used 
in the MC. We expect the first piece to dominate, but want to check what could be the magnitude of the unknown mismodeling.

* MC-only studies on a ttbar sample show that the jet response vs number of vertices in an event does not match our expectations from minbias data, and the difference is significantly larger than the L4 systematic for the effect of pileup on jet energies. This is not necessarily a problem, because the data could have the same behavior in ttbar events. (There is not enough statistical power to check in the data.) But the observed effect in $\mathrm{MC}$ is a good probe of the order of possible mismodeling.

* The observed jet response slope after jet corrections is $250 \mathrm{MeV} /$ jet/vertex. The nominal L4 systematic is $107 \mathrm{MeV} /$ jet/vertex. So we can estimate the importance of this order of mismodeling by scaling up the L4 systematic by a factor of 2.3 .

* An additional complication is that the L4 systematic is usually evaluated on e.g. ttop75, which as we know has the wrong luminosity profile. The L4 correction/systematic goes as the number of vertices minus one, so the scaling factor becomes $2.3 *(<$ Nint $>$ (data $)-1) /(<$ Nint $>(\mathrm{MC})-1)$.

- The larger of the two pieces (known mismodeling, unknown mismodeling) is quoted as the pileup systematic.

(11) Color Reconnection[21]

- The $\mathrm{CR}$ is done by evaluating the difference between ctopsd and ctopse samples. The systematic is direct difference like (MC generator). 


\section{References}

[1] Particle Data Group. Journal of Physics G, volume 37. IOP Publishing, July 2010.

[2] J F Arguin et al. Generic Jet Energy Corrections for Run II data used for the Winter Conferences. CDF Note 6280, CDF, 2003.

[3] Th CDF Collaboration. Top Dilepton Cross Section in $2 \mathrm{fb}-1$ using the DIL selection. CDF Note 9291, CDF, 2008.

[4] Ben Kilminster. Higgs Boson Searches at the Tevatron. ICHEP Talk, July 2010.

[5] O. Buchmueller et al. Predictions for Mt and Mw in Minimal Supersymmetric Models. Phys. Rev. D, 81:35009-1, 2010.

[6] R. Blair et al. The CDF II Detector Technical Design Report. CDF Collaboration, November 1996.

[7] Kristo M. Karr. Measurement of the Top Quark Mass by Application of the Dalitz-Goldstein Method to Dilepton Events. PhD thesis, Tufts University, 1999.

[8] Nima Arkani Hamed Savas Dimopoulos and Gia Dvali. The Hierarchy Problem and New Dimensions at a Millimeter. SLAC-PUB 7769, SLAC, 1998.

[9] B. Jayatilaka et al. Measurement of the Top Quark Mass in the Dilepton Channel in 2.0 fb-1 with the Matrix-Element Method. CDF Note 9098, CDF, 2008.

[10] Jian Tang Hyun Su Lee and Young Kee Kim. A Top Mass Measurement in Dilepton Channels with mt2. CDF Note 9748, CDF, 2009.

[11] R. H. Dalitz and G. Goldstein. Decay and Polarization Properties of the Top Quark. Phys. Rev. D, 45:1531, 1992.

[12] R.H.Dalitz G. Goldstein and K. Sliwa. On Observing the Top Quark Production at the Tevatron. CDF Note 1750, CDF, 1992.

[13] R.H.Dalitz G. Goldstein and K. Sliwa. Search for the Top-Antitop Events in the Semileptonic Mode. CDF Note 1751, CDF, 1992. 
[14] R.H.Dalitz G. Goldstein and K. Sliwa. Observing the Top Quark Production at the Fermilab Tevatron. Phys. Rev. D, 47:967, 1993.

[15] K. Sliwa. Search for the ttbar Events in the Lepton and Four Jets Final State. CDF Note 1993, CDF, 1993.

[16] K. Sliwa. A Non Black Box Approach to Kinematical Fitting and Its Application to Top Quark Search. CDF Note 3057, CDF, 1995.

[17] Top Mass Group. Systematics Prescriptions for the 2fb-1 Publications. webpage, 2008. http://www-cdf.fnal.gov/internal/physics/top/run2mass/systematics_08_add.html.

[18] The CDF Collaboration. Measurement of Exclusive B Lifetimes and Measurement of the Lifetime Ratios. CDF Note 8524, CDF, 2007.

[19] Jahred Adelman and Erik Brubaker. The Bootstrap Technique and its Application to Analyses in the Top Mass Group. CDF Note 9081, CDF, 2007.

[20] Top Mass Group. Systematics Prescriptions for Winter 2008. webpage, December 2008. http://www-cdf.fnal.gov/internal/physics/top/run2mass/systematics_08.html.

[21] G.Velev. Color Reconnection. private email, 2010. 